\title{
Metal-Nitrogen Multiple Bonds with Square-Planar Group 9 Transition Metal PNP Pincer Complexes
}

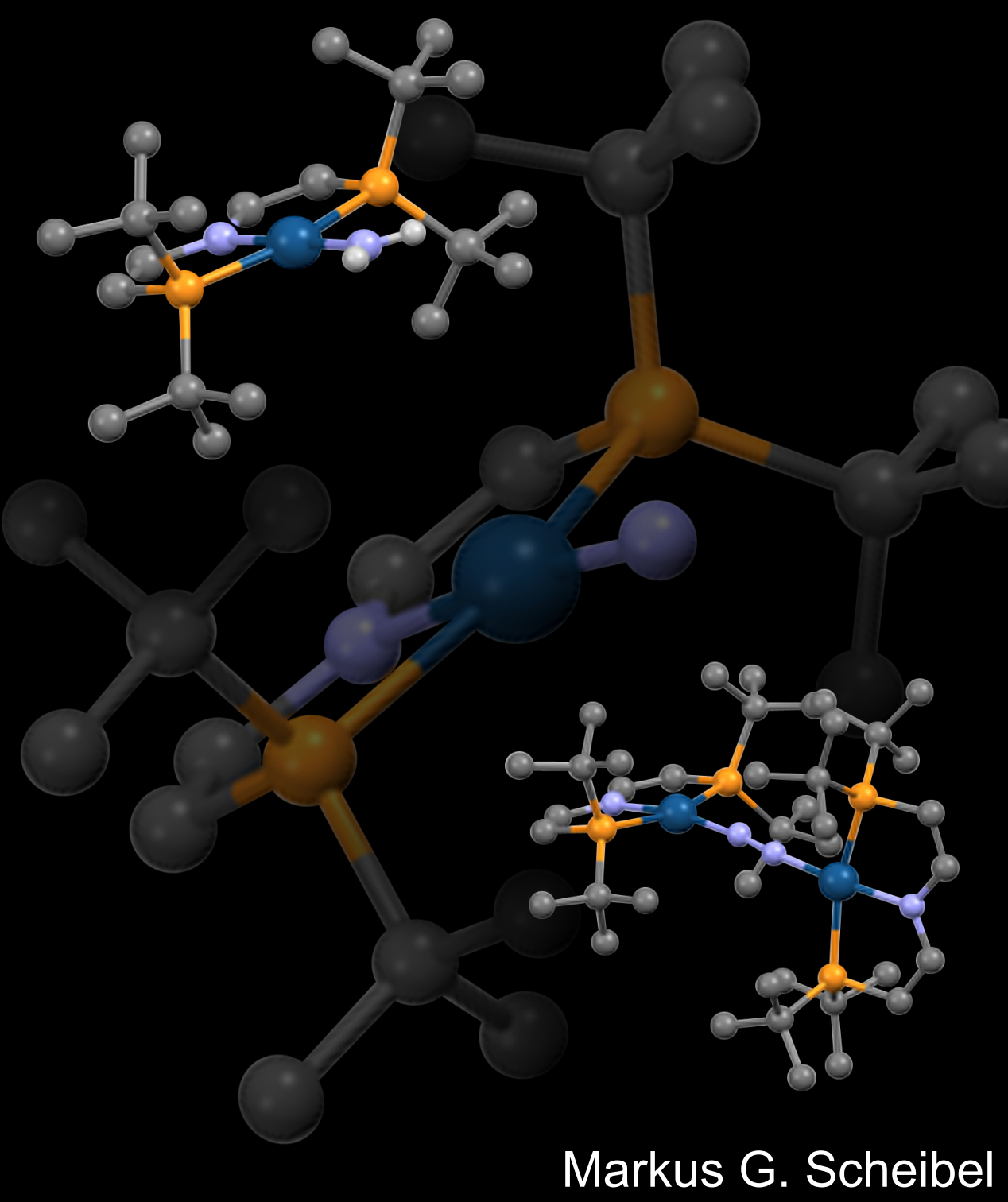




\section{Metal-Nitrogen Multiple Bonds with Square-Planar Group 9 Transition Metal PNP Pincer Complexes}

\section{Dissertation}

Zur Erlangung des mathematisch-naturwissenschaftlichen Doktorgrades

"DOCTOR RERUM NATURALIUM" der Georg-August-Universität Göttingen

im Promotionsprogramm Catalysis for Sustainable Synthesis (CaSuS) der Georg-August University School of Science (GAUSS)

vorgelegt von

M. Sc. Markus Scheibel

aus München

Göttingen, 2014 


\section{Betreunngsausschuss}

Prof. Dr. Sven Schneider,

Prof. Dr. Franc Meyer,

Institut für Anorganische Chemie der Georg-August-Universität-Göttingen.

Prof. Dr. Karsten Meyer,

Institut für Anorganische und Allgemeine Chemie der Friedrich-Alexander Universität

Erlangen-Nürnberg.

\section{Mitglieder Der PrüfungSKommission}

Referent:

Prof. Dr. Sven Schneider.

Co-Referent:

Prof. Dr. Franc Meyer.

Weitere Mitglieder der Prüfungskommission:

Prof. Dr. Karsten Meyer.

Institut für Anorganische und Allgemeine Chemie der Friedrich-Alexander Universität Erlangen-Nürnberg.

Prof. Dr. Dietmar Stalke,

Prof. Dr. Guido Clever,

Dr. INKE SIEWERT,

Institut für Anorganische Chemie der Georg-August-Universität Göttingen.

Tag der mündlichen Prüfung: 14.11.2014 
This is for my family.

Thanks for your support,

for all the love and trust.

Being with you,

means being home.

„It's still magic

even if you know how it's done."

TERry Pratchett 

This thesis is originated in the time between October 2010 and February 2011 at the Anorganisch-Chemisches Institut der Technischen Universität München, until Mai 2012 at the Departement Chemie und Pharmazie der

Friedrich-Alexander Universität Erlangen-Nürnberg and until September 2014 at the Institut für Anorganische Chemie der Georg-August-Universität Göttingen.

I am deeply indebted to my supervisor

Prof. Dr. Sven Schneider

Thank you Sven for welcoming me in your working group, the trust you put in me and my skills, for offering me a challenging and fascinating research project, the many opportunities to meet national and international researchers on conferences and the multitude of inspiring scientific discussions, that were crucial for the success of my thesis.

I also want to thank

Prof. Dr. h.c. mult Wolfgang A. Herrmann

for the opportunity to work in your chair and use the excellent infrastructure and

\section{Prof. Dr. Karsten Meyer}

for the time at your chair, for the access to the many analytical facilities, the stimulating scientific discussions and for appraising my thesis and being member of my committee. 
Furthermore, my special thanks go to:

My colleagues from the Schneider group Dr. Anja Friedrich, Dr. Jorge Torres Nieto, Dr. Alexander Marziale, Jenni Meiners, Dr. Markus Finger, Dr. Christian Würtele, Isabel Klopsch, Christian Volkmann, Markus Kinauer, Arne Glüer, Christoph Schiwek, Jan Gerkens, Dr. Paraskevi „Vivi“ Lagaditis and Florian Schendzielorz. Especially I want to thank Dr. Björn Askevold for the marvelous time in Erlangen and the many excellent scientific and non-scientific discussions.

I want to thank all my students for practical courses in the „work group Bob": Simon Meister, Oliver Langmar, Isabel Klopsch, Benjamin Freitag, Hauke Stürznickel, Christoph Schiewek, Kris Runge, Ingo Köhne, Julian Mögling, Teresa Sauter, Susanne Löffler, Alexander Brinkmeier, Mike Schütze, Alexander Paesch and Jan Gerkens. My special thanks go to Josh Abbenseth.

Prof. Dr. Bas de Bruin (EPR, DFT) for a very fruitful collaboration and the many scientific discussions.

Dr. Eberhardt Herdtweck, Dr. Frank W. Heinemann, Prof. Dr. Dietmar Stalke, Hilke Wolff, Peter Stollberg, Elena Carl, Lennard Krause and Dr. Christian Würtele (X-Ray diffraction) for their support.

Dr. Marat Khusniyarov, Prof. Dr. Ulrich Zenneck, Dr. Edward J. Reijerse and Dr. Claudia A. Stückl (EPR) for long endurance at late hours at the EPR spectrometer.

Kristian Dalle (ESI) for the many discussions about chemistry and life.

The members of the analytical laboratories of the Universities of Munich, Erlangen and Göttingen for their continuous efforts on measuring of highly sensitive samples.

The staff of the secretaries Irmgad Grötsch (Munich), Ulrike Hansl (Erlangen), and Dalila Griffin (Göttingen) for their help with administration affairs. Furthermore, I want to thank Dr. Hannah Steininger for support with the CaSuS graduate school.

Matthias Fichtl, Dr. Stefan Reindl, Dr. Dominik Jantke and all my friends from TU Munich for the unforgettable time during the undergraduate studies. Furthermore, I want to thank Dr. Florian Kiefer, Sabine Kiefer and all my friends for their support and understanding.

For the wonderful time we had together in and especially beyond the lab in Göttingen, I am deeply thankful to Isabel Klopsch. 


\section{Contents}

$\begin{array}{ll}\text { I. Introduction } & 1\end{array}$

1. Dinitrogen activation 2

$\begin{array}{ll}\text { 2. Terminal transition metal nitrides } & \mathbf{7}\end{array}$

2.1. Bonding and electronic structure . . . . . . . . . . . . . . . . . . 7

2.2. Reactivity ........................... . . 12

2.2.1. Nucleophilic vs. electrophilic reactivity . . . . . . . . . . . . . . . . 12

2.2.2. $\mathrm{N} \equiv \mathrm{N}$ cleavage and coupling . . . . . . . . . . . . . . . . . . . . 18

3. Synthesis of late transition metal nitrido complexes 22

3.1. Nitride precursors . . . . . . . . . . . . . . . . . . . . . . . . . . 22

3.2. Suitable platforms . . . . . . . . . . . . . . . . . . . . . . . . . . 23

3.3. Square-planar iridium(II) . . . . . . . . . . . . . . . . . . . 25

$\begin{array}{lr}\text { 4. Scope of this work } & 28\end{array}$

II. Results and Discussion 31

1. Square-planar iridium(I/II/III) platforms 32

1.1. Iridium $(\mathrm{II})$. . . . . . . . . . . . . . . . . . . . . . . . . . . . . 32

1.2. $\operatorname{Iridium}(\mathrm{III})$. . . . . . . . . . . . . . . . . . . . . . . . . . . . . . . . . . . 32

1.3. $\operatorname{Iridium}(\mathrm{I})$. . . . . . . . . . . . . . . . . . . . . . . . . . . . . 35

1.4. Reactivity of the iridium(I) PNP chloride . . . . . . . . . . . . . . . . . . 38

1.4.1. Iridium(I) carbonyl complex . . . . . . . . . . . . . . . . . . . . . 38

1.4.2. Oxidative addition to iridium(I) PNP chloride . . . . . . . . . . . . 41

1.4.3. $\mathrm{O}_{2}$ activation ...................... 43 
2. Square-planar iridium nitrido complexes 46

2.1. Iridium(II) PNP azide . . . . . . . . . . . . . . . . . . . . . . . . . 46

2.2. Iridium $(\mathrm{V})$ PNP nitride . . . . . . . . . . . . . . . . . . . . . . . 49

2.3. $\operatorname{Iridium}(\mathrm{IV})$ PNP nitride . . . . . . . . . . . . . . . . . . 53

2.3.1. Spectroscopic characterization . . . . . . . . . . . . . 53

2.3.2. DFT characterization . . . . . . . . . . . . . . 56

2.4. Iridium(I) PNP dinitrogen complexes . . . . . . . . . . . . . . . . . . 58

2.5. Investigations on the nitride coupling reaction . . . . . . . . . . . 61

2.6. C-H activation . . . . . . . . . . . . . . . . . . 63

3. Square-planar rhodium nitrido complex 65

3.1. Square-planar rhodium(II) chloro and azido complexes . . . . . . . . . . . 65

3.2. Open-shell rhodium nitride . . . . . . . . . . . . . . . . . . . 68

3.3. Reactivity of open-shell rhodium nitrido complex . . . . . . . . . . . . 72

4. Series of parent Ir amino, amido, imido and nitrido complexes $\mathbf{7 5}$

4.1. Parent iridium(II-III) amido and amino complexes . . . . . . . . . . . 77

4.2. Iridium imido complexes . . . . . . . . . . . . . . . . . . . . . . . . . . 84

4.3. Discussion . . . . . . . . . . . . . . . . . . . . . 96

4.3.1. HAT between iridium amide and nitride . . . . . . . . . . . . 97

4.3.2. Deprotonation of cationic iridium amide . . . . . . . . . . . . 100

4.4. Nitride coupling versus $\mathrm{H}$-atom abstraction . . . . . . . . . . . . . . 104

5. Chalcogenonitrosyl complexes of iridium 105

5.1. Synthesis . . . . . . . . . . . . . . . . . . . . . 105

5.2. IR spectroscopic characterization . . . . . . . . . . . . . . . 107

5.3. Discussion of the molecular structures . . . . . . . . . . . . . . . . 108

5.4. Discussion of the bonding model . . . . . . . . . . . . . . . . . 110

$\begin{array}{ll}\text { III. Conclusion } & 113\end{array}$

$\begin{array}{ll}\text { IV. Experimental Part } & 121\end{array}$

$\begin{array}{lr}\text { 1. Methods } & 122\end{array}$

1.1. General methods . . . . . . . . . . . . . . . . . . . . . . . 122 
1.2. Analytical methods . . . . . . . . . . . . . . . . . . . . . . 123

2. Starting material and ligand synthesis $\quad 128$

2.1. Reagents and starting materials . . . . . . . . . . . . . . . . . . . 128

2.1.1. Commercial available reagents and starting materials . . . . . . . . 128

2.1.2. Non-commercial available reagents and starting materials . . . . . 128

2.2. Modified procedures . . . . . . . . . . . . . . . . . . . . . . . 129

2.2.1. Bis(di-tert-butylphosphinoethyl)amine (1) . . . . . . . . . . 129

2.2.2. Bis(triphenylphosphoranylidene)ammonium azide . . . . . . . . . 130

3. Iridium(I-III) PNP plattforms 131

3.1. $\left[\operatorname{Ir}\left\{\mathrm{N}\left(\mathrm{CHCHP}^{t} \mathrm{Bu}_{2}\right)_{2}\right\} \mathrm{Cl}\right](3) \ldots \ldots \ldots \ldots$

3.2. $\left[\operatorname{Ir}\left\{\mathrm{N}\left(\mathrm{CHCHP}^{t} \mathrm{Bu}_{2}\right)_{2}\right\} \mathrm{Cl}\right] \mathrm{PF}_{6}(4) \ldots \ldots \ldots . \ldots \ldots . \ldots \ldots$

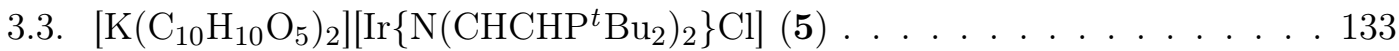

4. Small molecule activation by iridium(I) PNP 135

4.1. $\left[\operatorname{Ir}\left\{\mathrm{N}\left(\mathrm{CHCHP}^{t} \mathrm{Bu}_{2}\right)_{2}\right\}(\mathrm{CO})\right](6) \ldots \ldots \ldots \ldots$

4.2. $\left[\operatorname{Ir}\left\{\mathrm{N}\left(\mathrm{CHCHP}^{t} \mathrm{Bu}_{2}\right)_{2}\right\}(\mathrm{H})\left(\mathrm{C}_{6} \mathrm{H}_{5}\right)\right](\mathbf{8}) \ldots \ldots \ldots \ldots . \ldots \ldots$

4.3. $\left[\operatorname{Ir}\left\{\mathrm{N}\left(\mathrm{CHCHP}^{t} \mathrm{Bu}_{2}\right)_{2}\right\}\left(\mathrm{CH}_{3}\right) \mathrm{Cl}\right](\mathbf{9}) \ldots \ldots \ldots . \ldots \ldots$

4.4. $\left[\operatorname{Ir}\left\{\mathrm{N}\left(\mathrm{CHCHP}^{t} \mathrm{Bu}_{2}\right)_{2}\right\}\left(\mathrm{O}_{2}\right)\right](\mathbf{1 0}) \ldots \ldots \ldots \ldots$

$\begin{array}{ll}\text { 5. Iridium nitrido complexes } & 141\end{array}$

5.1. $\left[\operatorname{Ir}\left\{\mathrm{N}\left(\mathrm{CHCHP}^{t} \mathrm{Bu}_{2}\right)_{2}\right\}\left(\mathrm{N}_{3}\right)\right](\mathbf{1 1}) \ldots \ldots \ldots . \ldots \ldots 14 \ldots \ldots$

5.2. ${ }^{15 / 14} \mathrm{~N}^{14} \mathrm{~N}_{2-}\left[\operatorname{Ir}\left\{\mathrm{N}\left(\mathrm{CHCHP}^{t} \mathrm{Bu}_{2}\right)_{2}\right\}\left(\mathrm{N}_{3}\right)\right]\left({ }^{\mathbf{1 5} / \mathbf{1 4}} \mathrm{N}-\mathbf{1 1}\right) \ldots \ldots . . \ldots . . . . .142$

5.3. $\left[\operatorname{Ir}\left\{\mathrm{N}\left(\mathrm{CHCHP}^{t} \mathrm{Bu}_{2}\right)_{2}\right\}(\mathrm{N})\right] \mathrm{PF}_{6}(\mathbf{1 2}) \ldots \ldots \ldots \ldots . \ldots \ldots . \ldots \ldots$

5.4. $\left[\operatorname{Ir}\left\{\mathrm{N}\left(\mathrm{CHCHP}^{t} \mathrm{Bu}_{2}\right)_{2}\right\}(\mathrm{N})\right](\mathbf{1 3}) \ldots \ldots \ldots \ldots$

5.5. $\quad\left[\operatorname{Ir}\left\{\mathrm{N}\left(\mathrm{CHCHP}^{t} \mathrm{Bu}_{2}\right)_{2}\right\}\left(\mathrm{N}_{2}\right)\right](7) \ldots \ldots \ldots \ldots \ldots . \ldots \ldots \ldots$

5.6. $\left[\operatorname{Ir}\left\{\mathrm{N}\left(\mathrm{CHCHP}^{t} \mathrm{Bu}_{2}\right)_{2}\right\}\right]_{2}\left(\mathrm{~N}_{2}\right)(\mathbf{1 4}) \ldots \ldots \ldots \ldots \ldots$

5.7. Coupling of $\mathbf{1 3}$ in the presence of 1,4 -cyclohexadiene . . . . . . . . . 147

$\begin{array}{lr}\text { 6. Rhodium nitrido complexes } & \mathbf{1 4 8}\end{array}$

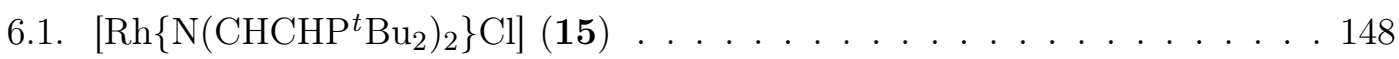

6.2. $\left[\mathrm{Rh}\left\{\mathrm{N}\left(\mathrm{CHCHP}^{t} \mathrm{Bu}_{2}\right)_{2}\right\}\left(\mathrm{N}_{3}\right)\right](\mathbf{1 6}) \ldots \ldots \ldots . \ldots \ldots$

6.3. ${ }^{15 / 14} \mathrm{~N}^{14} \mathrm{~N}_{2-}\left[\mathrm{Rh}\left\{\mathrm{N}\left(\mathrm{CHCHP}^{t} \mathrm{Bu}_{2}\right)_{2}\right\}\left(\mathrm{N}_{3}\right)\right]\left({ }^{\mathbf{1 5} / \mathbf{1 4}} \mathrm{N}-\mathbf{1 6}\right) \ldots \ldots \ldots . \ldots 150$

6.4. $\left[\mathrm{Rh}\left\{\mathrm{N}\left(\mathrm{CHCHP}^{t} \mathrm{Bu}_{2}\right)_{2}\right\}(\mathrm{N})\right](\mathbf{1 7}) \ldots \ldots \ldots \ldots$

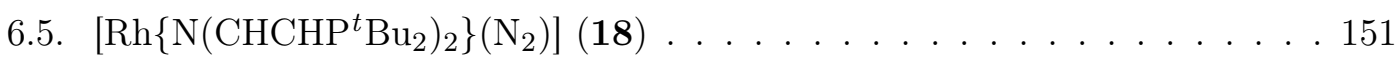

6.6. $\left[\mathrm{Rh}\left\{\mathrm{N}\left(\mathrm{CHCHP}^{t} \mathrm{Bu}_{2}\right)_{2}\right\}\right]_{2}\left(\mathrm{~N}_{2}\right)(\mathbf{1 9}) \ldots \ldots \ldots \ldots . \ldots \ldots$ 
6.7. Photolysis of $\mathbf{1 6}$ in the presence of 1,4 -cyclohexadiene . . . . . . . . 153

7. Amino and amido complexes of iridium 154

7.1. $\left[\operatorname{Ir}\left\{\mathrm{N}\left(\mathrm{CHCHP}^{t} \mathrm{Bu}_{2}\right)_{2}\right\}\left(\mathrm{NH}_{2}\right)\right](\mathbf{2 4}) \ldots \ldots \ldots \ldots$

7.2. $\left[\operatorname{Ir}\left\{\mathrm{N}\left(\mathrm{CHCHP}^{t} \mathrm{Bu}_{2}\right)_{2}\right\}\left(\mathrm{NH}_{2}\right)\right] \mathrm{PF}_{6}(\mathbf{2 1}) \ldots \ldots \ldots \ldots \ldots \ldots$

7.3. $\left[\operatorname{Ir}\left\{\mathrm{N}\left(\mathrm{CHCHP}^{t} \mathrm{Bu}_{2}\right)_{2}\right\}\left(\mathrm{NH}_{3}\right)\right]\left[\mathrm{B}\left(\mathrm{C}_{6} \mathrm{H}_{3}\left(\mathrm{CF}_{3}\right)_{2}\right)_{4}\right](\mathbf{2 0}) \ldots \ldots \ldots . \ldots . \ldots . . \ldots 6$

7.4. Conversion of 13 with TEMPO-H . . . . . . . . . . . . . . . . 157

7.5. Coupling of $\mathbf{1 3}$ in the presence of 2,4,6-tri-tert-butylphenol . . . . . . 158

7.6. Conversion of 24 with TEMPO . . . . . . . . . . . . . . . . . . 158

7.7. Reaction of $\mathbf{2 4}$ with tri-tert-butylphenoxyl radical . . . . . . . . . . . 158

7.8. Conversion of 21 with $\mathrm{K}\left[\mathrm{N}\left(\mathrm{SiMe}_{3}\right)_{2}\right] \ldots \ldots$. . . . . . . . . . 159

8. Chalcogenonitrosyl complexes of iridium 160

8.1. $\left[\operatorname{Ir}\left\{\mathrm{N}\left(\mathrm{CHCHP}^{t} \mathrm{Bu}_{2}\right)_{2}\right\}(\mathrm{NO})\right] \mathrm{PF}_{6}(\mathbf{2 5}) \ldots \ldots \ldots \ldots$

8.2. $\left[\operatorname{Ir}\left\{\mathrm{N}\left(\mathrm{CHCHP}^{t} \mathrm{Bu}_{2}\right)_{2}\right\}(\mathrm{NS})\right] \mathrm{PF}_{6}(\mathbf{2 6}) \ldots \ldots \ldots \ldots 16 \ldots \ldots$

8.3. $\left[\operatorname{Ir}\left\{\mathrm{N}\left(\mathrm{CHCHP}^{t} \mathrm{Bu}_{2}\right)_{2}\right\}(\mathrm{NSe})\right] \mathrm{PF}_{6}(\mathbf{2 7}) \ldots \ldots \ldots$

$\begin{array}{ll}\text { V. Literature } & 165\end{array}$

$\begin{array}{ll}\text { VI. Appendix } & 185\end{array}$

$\begin{array}{ll}\text { 1. Index of all numerated compounds } & 186\end{array}$

$\begin{array}{lr}\text { 2. List of Abbreviations } & 188\end{array}$

3. Crystallographic Details 190

3.1. $\left[\operatorname{Ir}\left\{\mathrm{N}\left(\mathrm{CHCHP}^{t} \mathrm{Bu}_{2}\right)_{2}\right\} \mathrm{Cl}\right] \mathrm{PF}_{6}(4) \ldots \ldots \ldots . \ldots \ldots$

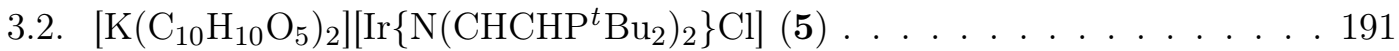

3.3. $\left[\operatorname{Ir}\left\{\mathrm{N}\left(\mathrm{CHCHP}^{t} \mathrm{Bu}_{2}\right)_{2}\right\}(\mathrm{CO})\right](6) \ldots \ldots . \ldots \ldots . \ldots \ldots$

3.4. $\left[\operatorname{Ir}\left\{\mathrm{N}\left(\mathrm{CHCHP}^{t} \mathrm{Bu}_{2}\right)_{2}\right\}\left(\mathrm{N}_{2}\right)\right](7) \ldots \ldots \ldots \ldots$

3.5. $\left[\operatorname{Ir}\left\{\mathrm{N}\left(\mathrm{CHCHP}^{t} \mathrm{Bu}_{2}\right)_{2}\right\}(\mathrm{H})\left(\mathrm{C}_{6} \mathrm{H}_{5}\right)\right](8) \ldots \ldots \ldots \ldots$

3.6. $\left[\operatorname{Ir}\left\{\mathrm{N}\left(\mathrm{CHCHP}^{t} \mathrm{Bu}_{2}\right)_{2}\right\}\left(\mathrm{O}_{2}\right)\right](\mathbf{1 0}) \ldots \ldots \ldots \ldots . \ldots \ldots . \ldots \ldots$

3.7. $\left[\operatorname{Ir}\left\{\mathrm{N}\left(\mathrm{CHCHP}^{t} \mathrm{Bu}_{2}\right)_{2}\right\} \mathrm{N}\right] \mathrm{OTf}(\mathbf{1 2}) \ldots \ldots \ldots \ldots$

3.8. $\left[\operatorname{Ir}\left\{\mathrm{N}\left(\mathrm{CHCHP}^{t} \mathrm{Bu}_{2}\right)_{2}\right\}\right]_{2}\left(\mathrm{~N}_{2}\right)(\mathbf{1 4}) \ldots \ldots \ldots \ldots$

3.9. $\left[\mathrm{Rh}\left\{\mathrm{HN}\left(\mathrm{CH}_{2} \mathrm{CH}_{2} \mathrm{P}^{t} \mathrm{Bu}_{2}\right)_{2}\right\}\left(\mathrm{PPh}_{3}\right)\right] \mathrm{Cl} \ldots \ldots \ldots \ldots$ 
3.10. $\left[\mathrm{Rh}\left\{\mathrm{N}\left(\mathrm{CHCHP}^{t} \mathrm{Bu}_{2}\right)_{2}\right\} \mathrm{Cl}\right](\mathbf{1 5}) \ldots \ldots \ldots \ldots$

3.11. $\left[\mathrm{Rh}\left\{\mathrm{N}\left(\mathrm{CHCHP}^{t} \mathrm{Bu}_{2}\right)_{2}\right\}\left(\mathrm{N}_{2}\right)\right](\mathbf{1 8}) \ldots \ldots \ldots . \ldots \ldots$

3.12. $\left[\operatorname{Ir}\left\{\mathrm{N}\left(\mathrm{CHCHP}^{t} \mathrm{Bu}_{2}\right)_{2}\right\}\left(\mathrm{NH}_{3}\right)\right]\left[\mathrm{BF}_{4}\right](\mathbf{2 0}) \ldots \ldots \ldots . \ldots \ldots$

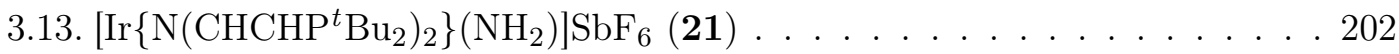

3.14. $\left[\operatorname{Ir}\left\{\mathrm{N}\left(\mathrm{CHCHP}^{t} \mathrm{Bu}_{2}\right)_{2}\right\}\left(\mathrm{NH}_{2}\right)\right](\mathbf{2 4}) \ldots \ldots \ldots \ldots . \ldots \ldots . \ldots \ldots$

3.15. $\left[\operatorname{Ir}\left\{\mathrm{N}\left(\mathrm{CHCHP}^{t} \mathrm{Bu}_{2}\right)_{2}\right\}(\mathrm{NO})\right] \mathrm{PF}_{6}(\mathbf{2 5}) \ldots \ldots \ldots \ldots . \ldots \ldots$

3.16. $\left[\operatorname{Ir}\left\{\mathrm{N}\left(\mathrm{CHCHP}^{t} \mathrm{Bu}_{2}\right)_{2}\right\}(\mathrm{NS})\right] \mathrm{PF}_{6}(\mathbf{2 6}) \ldots \ldots \ldots \ldots$

3.17. $\left[\operatorname{Ir}\left\{\mathrm{N}\left(\mathrm{CHCHP}^{t} \mathrm{Bu}_{2}\right)_{2}\right\}(\mathrm{NSe})\right] \mathrm{PF}_{6}(\mathbf{2 7}) \ldots \ldots \ldots \ldots$

$\begin{array}{ll}\text { 4. List of scientific contributions } & 207\end{array}$

$\begin{array}{ll}\text { 5. Curriculum vitae } & 209\end{array}$ 

Part I.

\section{Introduction}




\section{Dinitrogen activation}

Nitrogen is besides carbon, hydrogen and oxygen one of the main elements found in organic molecules and an important building block for molecular architecture and reactivity. In nature, it appears mainly in form of the diatomic molecule dinitrogen $\left(\mathrm{N}_{2}\right)$. The two nitrogen atoms of dinitrogen are connected via a triple bond ${ }^{[1]}$ formed by one molecular orbital (MO) with $\sigma$ - and two MOs with $\pi$-symmetry. The resulting strong $\mathrm{N} \equiv \mathrm{N}$ bond $(\mathrm{BDE}=941 \mathrm{~kJ} / \mathrm{mol})$ is the reason for the high thermodynamic stability of $\mathrm{N}_{2}$. In addition, $\mathrm{N}_{2}$ exhibits a large energy difference of $10.82 \mathrm{eV}$ between the highest occupied molecular orbital (HOMO) possessing $\sigma$-symmetry, and the lowest unoccupied molecular orbital (LUMO), which is $\pi$-symmetric. ${ }^{[2]}$ The cleavage of the first bond upon formation of a formal $\mathrm{N}-\mathrm{N}$ double bond requires the major part of the energy $(410 \mathrm{~kJ} / \mathrm{mol})$ needed for $\mathrm{N}_{2}$ splitting, and hence, results in a high kinetic barrier for the transformation of $\mathrm{N}_{2} \cdot{ }^{[3]}$ Both kinetic and thermodynamic stability are the reason for the inertness of $\mathrm{N}_{2}$, promoting its use e.g. as an inert gas, and hinders its direct utilization as starting material for the synthesis of fertilizers or organic materials. ${ }^{[1]}$

A synthetically suitable nitrogen source for bio- and industrial utilization is ammonia $\left(\mathrm{NH}_{3}\right)$ and its formation is covered by the term 'nitrogen fixation'. ${ }^{[4]}$ The synthesis of $\mathrm{NH}_{3}$ out of the elements (equation 1.1) can be performed catalytically, due to the negative heat of formation of $\mathrm{NH}_{3}\left(\Delta H_{f}^{0}=-46.2 \mathrm{KJ} / \mathrm{mol}\right)$. Despite the tremendous importance of the $\mathrm{NH}_{3}$ synthesis for nature and mankind, only two main processes exist for the world's $\mathrm{NH}_{3}$ production today. ${ }^{[5]}$

$$
\mathrm{N}_{2}+3 \mathrm{H}_{2} \rightarrow 2 \mathrm{NH}_{3}
$$

The process responsible for nitrogen fixation in vivo is performed via an enzyme class called 'nitrogenase'. ${ }^{[6]}$ The site of $\mathrm{N}_{2}$-fixation in the most active, the Fe-Mo nitrogenase was first structurally characterized 1992 by Rees and coworkers using X-ray diffraction studies and turned out to be a cluster of seven iron and one molybdenum atoms bridged by ten sulfur atoms (FeMo-co). ${ }^{[7]} \mathrm{A}$ higher resolved structure indicated one light atom 
$(\mathrm{C}, \mathrm{N}, \mathrm{O})$ in the center of the cluster. ${ }^{[8]}$ Its identity was revealed as interstitial carbide spectroscopically by X-ray emission spectroscopy ${ }^{[9]}$ and electron spin echo envelope modulation $^{[10]}$, and also by X-ray diffraction with higher resolution one decade later ${ }^{[10]}$. There are two different pathways proposed for the hydrogenation mechanisms of $\mathrm{N}_{2}$ by nitrogenase (figure I.1.1): The alternating pathway entails the reduction and protonation of metal bound $\mathrm{N}_{2}$ (most likely $\mathrm{Fe}$ ) under formation of a diazene species, that is further converted into metal bound hydrazine. The distal mechanism covers full reduction and protonation of the nitrogen atom in $\beta$-position to the metal center and is anticipated to proceed upon reduction of Mo bound $\mathrm{N}_{2} \cdot{ }^{[6,11]}$

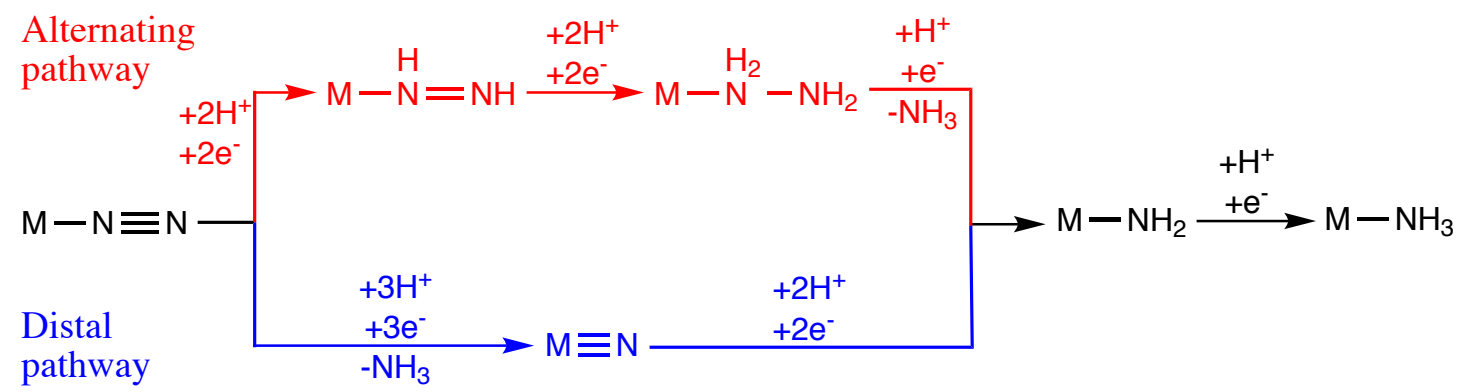

Figure I.1.1.: Proposed mechanisms for the reduction of $N_{2}$ at nitrogenase ( $M=M o$ or $\mathrm{Fe}) .[6,11]$

The latter mechanism is in close analogy to the homogeneous system presented by Schrock and Yandulov in 2003: ${ }^{[12]}$ Successive catalytic protonation and reduction of metal coordinated $\mathrm{N}_{2}$ at the distal $\mathrm{N}$-atom was achieved by a mononuclear Mo complex (figure I.1.2, left). Six turn overs were observed in total with an overall yield of $63-66 \%$ of $\mathrm{NH}_{3}$ with respect to the amount of reducing agent applied. ${ }^{[13]}$ The cleavage of the $\mathrm{N}-\mathrm{N}$ bond occurs after a total reduction by three electrons and addition of three protons to the $\mathrm{N}$ atom in $\beta$-position to the Mo center (distal N atom). ${ }^{[14]}$ Nishibayashi and coworkers reported higher catalytic activities for a different Mo complex (figure I.1.2, middle) also by addition of proton and electron sources. ${ }^{[15]}$ Remarkably, Peters and coworkers reported an Fe based catalytic system (figure I.1.2, right) with even better performance under similar conditions. The demonstration of an Fe based molecular catalyst questions the nature of the metal for $\mathrm{N}_{2}$ fixation in nitrogenase. ${ }^{[16]}$ 


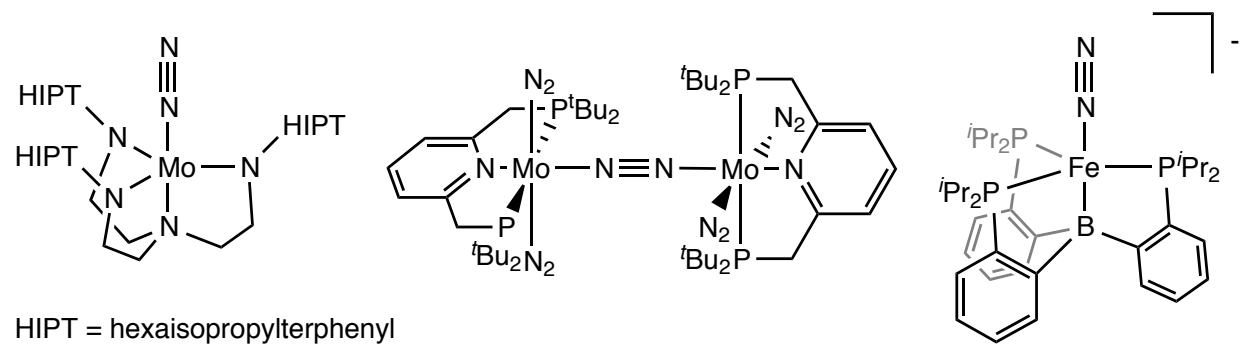

Figure I.1.2.: Catalytically active complexes for the reduction of $\mathrm{N}_{2}$ to $\mathrm{NH}_{3}$ in the presence of reducing agent and proton source.

The industrial production of $\mathrm{NH}_{3}$ is preformed heterogeneously catalyzed by reaction of $\mathrm{N}_{2}$ with $\mathrm{H}_{2}$ developed by Fritz Haber, applied on industrial scale by Carl Bosch and is known as the Haber-Bosch-Process. The produced quantity of $175 \cdot 10^{6}$ ton/year of $\mathrm{NH}_{3}$ is only exceeded by the production of sulfuric acid. The tremendous demand of $\mathrm{NH}_{3}$ in combination with the harsh reaction conditions $\left(480{ }^{\circ} \mathrm{C}, 400\right.$ bar) required for its industrial production leads to an overall consumption of $1.4 \%$ of the global energy and hence, demonstrates the importance of nitrogen fixation for modern economy. ${ }^{[17]}$

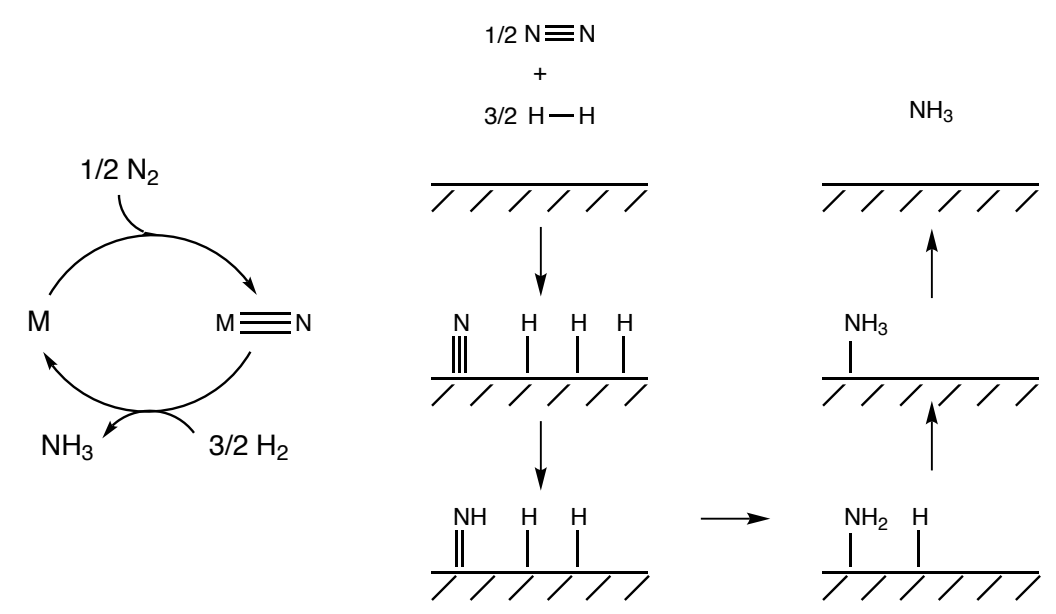

Figure I.1.3.: left: Two principle steps of dinitrogen fixation. $N_{2}$ splitting into terminal nitrido complexes and conversion of the nitrides with $\mathrm{H}_{2}$ under formation of $\mathrm{NH}_{3}$. right: Accepted mechanism for $\mathrm{NH}_{3}$ synthesis on iron surface following dissociative chemisorption of the reactant gases $\mathrm{N}_{2}$ and $\mathrm{H}_{2}$ and the conversion of resulting surface bound nitrides with chemisorbed H-atoms. 
The accepted mechanism for the Haber-Bosch-process can be divided into two principle parts (figure I.1.3): Dissociative chemisorption of $\mathrm{N}_{2}$ to surface bond nitrides ${ }^{[18]}$ and their conversion with $\mathrm{H}_{2}{ }^{[19]}$. A model reaction for the first part, namely the cleavage of $\mathrm{N}_{2}$ into terminal nitrides at ambient temperatures was first demonstrated by Cummins and coworkers in 1995 by conversion of $\mathrm{N}_{2}$ with a three-coordinate triamido $\mathrm{Mo}^{\mathrm{III}}$ complex, resulting in a four-coordinate $\mathrm{Mo}{ }^{\mathrm{VI}}$ nitrido complex (scheme I.1.1 top). ${ }^{[20]}$ Later on, $\mathrm{N}_{2}$ cleavage into terminal nitrides with tricoordinate early transition metal platforms was achieved with Mo supported by similar ancillary amido-ligands ${ }^{[21,22]}$, and could be further demonstrated using mixed $\mathrm{Nb} /$ Mo systems ${ }^{[23,24]}$. Except one example using Os, ${ }^{[25]}$ $\mathrm{N}_{2}$ splitting reactions resulting in defined terminal nitrido complexes were performed with early transition-metals owing to the high thermodynamic stabilities of the resulting nitrido complexes. ${ }^{[26]}$ Recently, cleavage of $\mathrm{N}_{2}$ was demonstrated using a five coordinated Mo complex ${ }^{[22]}$ and even more remarkable, under utilization of a Re complex ${ }^{[27]}$.

\section{Step: Splitting $\mathbf{N}_{2}$ into terminal nitrido complex}

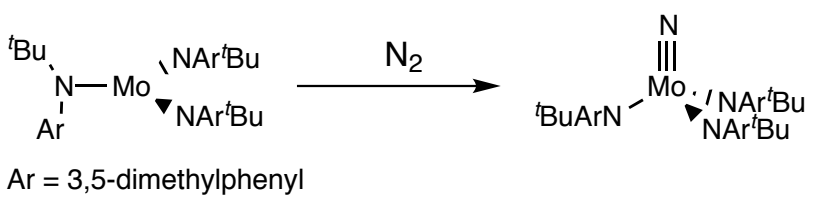

2. Step: Hydrogenation of terminal nitrido complex

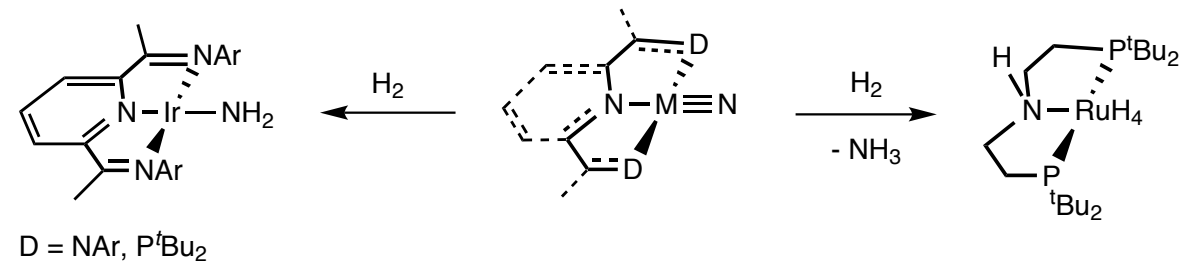

Scheme I.1.1: First example of dinitrogen splitting into a well defined terminal nitrido complex (top), and examples for conversion of terminal nitrido ligands with $\mathrm{H}_{2}$.

The second part of the nitrogen fixation cycle, namely the conversion of a terminal nitrido moiety with $\mathrm{H}_{2}$ was only demonstrated with late transition metal nitrido complexes (scheme I.1.1 bottom): Insertion of an iridium nitrido complex into $\mathrm{H}_{2}$ under formation of a parent iridium amido complex was reported by Burger and coworkers. ${ }^{[28]}$ Furthermore, full hydrogenolysis towards ammonia was achieved using a ruthenium nitride. In this case, metal-ligand cooperativity was found to be crucial for $\mathrm{H}_{2}$ activation. ${ }^{[29]}$ Today, there is only one system reported to achieve the substoichiometric conversion of 
$\mathrm{N}_{2}$ with $\mathrm{H}_{2}$ to $\mathrm{NH}_{3}$ under ambient conditions using a bis-zirconocene dimer. ${ }^{[30]}$ Recently, a tetra-nuclear iron dinitrogen complex was reported undergoing the formation $\mathrm{NH}_{3}$ in the presence of $\mathrm{H}_{2} \cdot{ }^{[31]}$ However, reinvestigations on the reaction revealed that the conversion was only attributed to the presence of acid. ${ }^{[32]}$

These examples demonstrate well the challenge of $\mathrm{N}_{2}$ fixation to chemists. With respect to the high energy consumption of industrial nitrogen fixation, the formation of $\mathrm{NH}_{3}$ by hydrogenation of $\mathrm{N}_{2}$ or metal nitrido complexes is still a subject of high importance for research today. Furthermore, the high temperatures required for heterogeneously catalyzed $\mathrm{NH}_{3}$ formation result in low yields and a catalytic system operating at lower temperatures is a desirable goal in modern research. However, the commercial HaberBosch process is one of the energetically most optimized processes in industry, ${ }^{[33]}$ and a 'renaissance' of the industrial $\mathrm{NH}_{3}$ production driven by a homogenous catalyst is rather unlikely. Nonetheless, the synthesis of a broad scope of nitrogen containing compounds like fertilizers, pharmaceuticals, basic and fine chemicals directly from $\mathrm{N}_{2}$ without the full protonation to $\mathrm{NH}_{3}$ or $\mathrm{NH}_{4}{ }^{+}$is relevant for research and industry. [34,35]

Note that the direct use of $\mathrm{NH}_{3}$ as nitrogen source for the formation of organic compounds is challenging attributed to the strong $\mathrm{N}-\mathrm{H}$ bond dissociation enthalpy $\left(\mathrm{BDE}\left(\mathrm{NH}_{3}\right)=\right.$ $107 \mathrm{kcal} / \mathrm{mol})^{[36]}$ and the high affinity of transition-metal catalysts for the formation of inactive Werner-type amine-complexes. ${ }^{[37]} \mathrm{C}-\mathrm{N}$ coupling mediated by transition metal nitrene species is an evolving field of research in organic chemistry. ${ }^{[38,39]}$ Providing a pathway to generate these nitrenes directly from $\mathrm{N}_{2}$ would bypass the energy demanding $\mathrm{NH}_{3}$ formation and represents an alternative approach for $\mathrm{N}_{2}$ fixation.

On the other hand, $\mathrm{NH}_{3}$ exhibits a high energy density. In addition, the existing technologies for secure storage, handling and distribution of $\mathrm{NH}_{3}$ promotes its use as a fuel e.g. for applications in a direct ammonia fuel cell. ${ }^{[33,40,41]}$ Anodic oxidation of $\mathrm{NH}_{3}$ to $\mathrm{N}_{2}$ with release of three protons and three electrons, for example derived by cathodic reduction of $\mathrm{O}_{2}$ to $\mathrm{H}_{2} \mathrm{O}$, represents a sustainable and carbon-free alternative for energy transformation and storage. ${ }^{40]}$ The understanding of the individual oxidation and deprotonation steps is crucial for the design of an appropriate catalyst. 


\section{Terminal transition metal nitrides}

\subsection{Bonding and electronic structure}

Lone pairs localized in $p$-orbitals of coordinated ligands, e.g. terminal oxo or nitrido ligands, are in $\pi$-symmetry with respect to the metal ligand bond. Their interaction with these $\pi$-symmetric metal $d$-orbitals can either be repulsive $d \pi / p \pi$ interactions when the metals $d$-orbitals are filled (figure I.2.1 left), or a multiple bond can result with empty $\pi$-symmetric $d$-orbitals (figure I.2.1 right). ${ }^{[42,43]}$ Hence, these ligands are characterized as strong $\pi$-donors and rise the energy of the $\pi$-symmetric $d$-orbitals. ${ }^{[43,44]}$

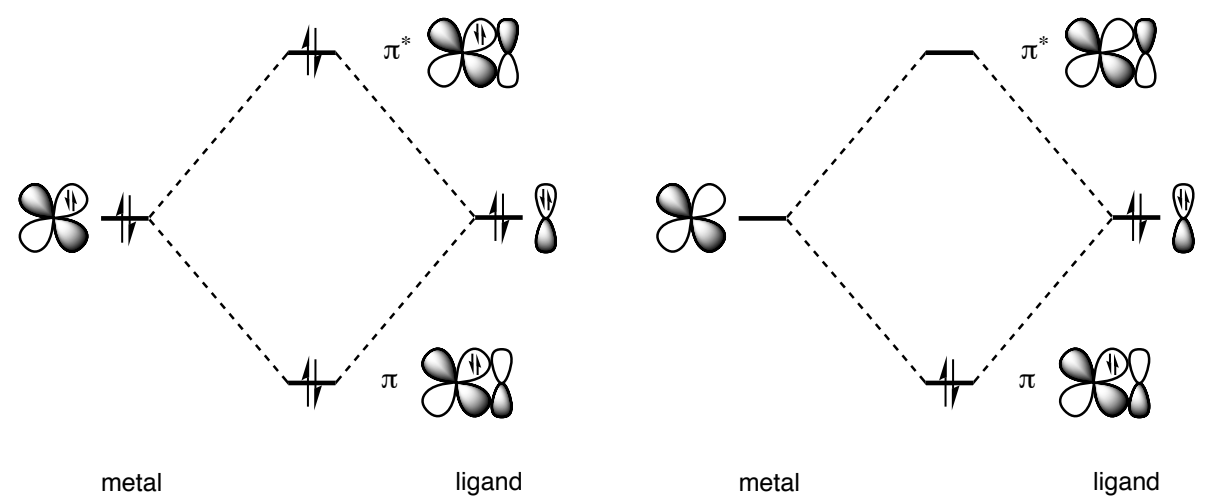

Figure I.2.1.: Interactions of a metal with a ligand bearing lone pairs. Repulsive $d \pi / p \pi$ interactions (left) when the $\pi$-symmetric metals $d$-orbitals are filled and formation of a multiple bond with empty $\pi$-symmetric d-orbitals (right).

The frontier-orbital splitting scheme for the octahedral vanadyl ion was established by Gray and Ballhausen ${ }^{[45]}$ and can be adopted for octahedral nitrido complexes. ${ }^{[42,46-50]}$ Attaching of a nitrido ligand in $z$-direction to an octahedral complex bearing solely $\sigma$ - 
donors as ancillary ligands results in a $d$-orbital splitting of $\left(d_{x y}\right)\left(d_{x z}, d_{x z}\right)\left(d_{x^{2}-y^{2}}\right)\left(d_{z^{2}}\right)$ (figure I.2.2 middle). The $d_{x y}$ and $d_{x^{2}-y^{2}}$ orbitals are non-bonding with respect to the nitrido ligand, the $d_{z^{2}}$ orbital derives from the $\sigma$-anti-bonding orbital combination and the $d_{x z}$ and $d_{y z}$ overlap with the nitrides $p_{x}$ and $p_{y}$ orbital resulting in the $\pi$-anti-bonding MOs. ${ }^{[51]}$ Filling of all bonding and non-bonding orbitals affords a total of 2 electrons and leads to a $\left(d_{x y}\right)^{2}\left(d_{x z}, d_{x z}\right)\left(d_{x^{2}-y^{2}}\right)\left(d_{z^{2}}\right)$ electronic configuration. Addition of more electrons results in occupation of $\mathrm{M}-\mathrm{N} \pi^{*}$-anti-bonding orbitals, reduces the $\mathrm{M}-\mathrm{N}$ bond order and hence destabilizes the complex. ${ }^{43,52]}$

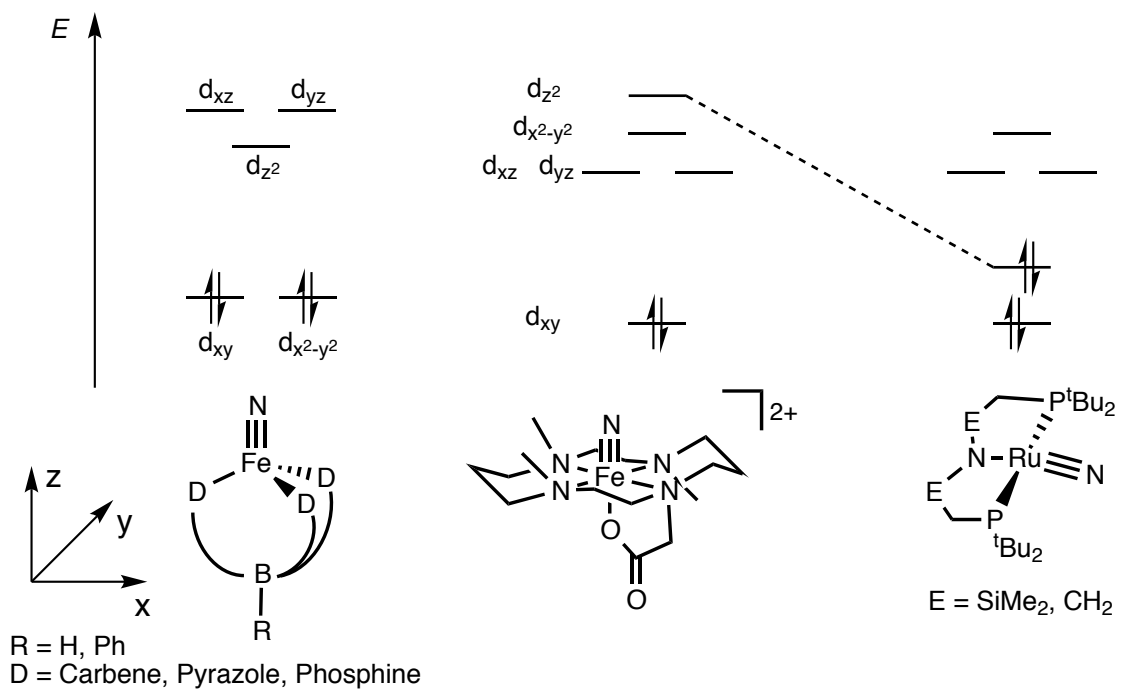

Figure I.2.2.: Qualitative frontier orbital splitting scheme and representative examples for nitrido complexes in threefold (left), fourfold (middle) and twofold (right) symmetry demonstrating the isolobal relationship between octahedral $d^{2}$ and square-planar $d^{4}$ nitrido complexes.

These simplified frontier orbital considerations explain well the high stability of transition metal complexes with multiply bonded ligands in a $d^{n}$ electronic configuration with $\mathrm{n} \leq 2$, and is the reason for the large number of reported octahedral $\mathrm{Re}^{\mathrm{V}}, \mathrm{Os}^{\mathrm{VI}}$ and $\mathrm{Ru} \mathrm{VI}^{\mathrm{V}}$ nitrido complexes. ${ }^{[48,53,54]}$

In contrast, the first terminal nitrido complex of iron was identified by observation of characteristic bands attributable to the $\mathrm{Fe} \equiv \mathrm{N}$ stretch after laser photolysis of $\mathrm{Fe}$ porphyrinato azido complexes via resonance raman spectroscopy. ${ }^{[55,56]}$ Further investigations on the electronic structures of Fe nitrido complexes were provided by Wieghardt and coworkers, 
who reported an $\mathrm{Fe}^{\mathrm{V}}$ nitride as the transient intermediate after photolysis of octahedral Fe azido complexes. ${ }^{[57]}$ The spin doublet ground-state of these systems supports full occupation of the non-bonding orbitals and one additional electron localized within the $\mathrm{Fe}-\mathrm{N} \pi^{*}$ orbitals, as it is suggested by frontier orbital considerations. ${ }^{[58]}$ Even more remarkable, photolysis of an $\mathrm{Fe}^{\mathrm{III}}$ azido complex allowed for the spectroscopic characterization of am Fe ${ }^{\mathrm{VI}}$ nitrido complex, which is, besides the ferrate anion $\left(\left[\mathrm{FeO}_{4}\right]^{2-}\right)$, the second example of Fe in the formal oxidation state of +VI (figure I.2.2 middle). ${ }^{59]}$

Thermally stable nitrido complexes are obtained when all $\pi$-anti-bonding orbitals are empty and the bonding and non-bonding orbitals are fully occupied. Hence, stable nitrides with a $d^{n}$ electron count $(n>2)$ requires additional non-bonding orbitals lower in energy than the $\mathrm{M} \equiv \mathrm{N} \pi^{*}$ orbitals. Upon changing from octahedral to tetrahedral coor-

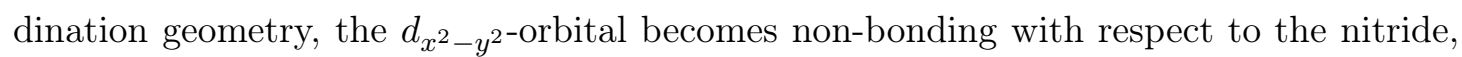
and therefore becomes lower in energy (figure I.2.2 left).
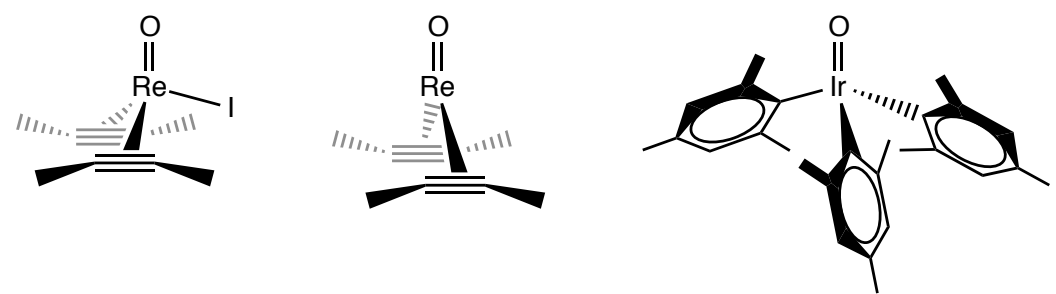

Figure I.2.3.: First terminal oxo complexes with a $d^{4}$ (left) and $d^{6}$ (middle) electron configuration and first terminal oxo complex beyond group 8 (right).

Accordingly, the synthesis and structural characterization of the first terminal oxo complex $\left[\mathrm{Re}^{\mathrm{III}}(\mathrm{MeC} \equiv \mathrm{CMe})_{2} \mathrm{I}(\mathrm{O})\right]$ with a $d^{4}$ electron count was reported by Mayer and coworkers (figure I.2.3 left). Investigations on the electronic structure revealed full occupation of two non-bonding $d$-orbitals with respect to the oxo ligand. ${ }^{[60]}$ Upon two electron reduction and release of $\mathrm{I}^{-}$, three coordinate $\mathrm{Re}^{\mathrm{I}}$ oxo complex $\left[\mathrm{Re}^{\mathrm{I}}(\mathrm{RC} \equiv \mathrm{CR})_{2}(\mathrm{O})\right]^{-}$with a $d^{6}$ electron count was isolated. This complex exhibits significant lowering of the Re-O bond order due to singly occupation of two Re-O $\pi^{*}$-orbitals (figure I.2.3 middle). ${ }^{[61]}$ Even more remarkably, the terminal oxo complex of iridium, $\left[\operatorname{Ir}(\mathrm{Mes})_{3}(\mathrm{O})\right](\mathrm{Mes}=2,4,6$ trimethylphenyl) reported by Wilkinson and coworkers is the first fully characterized example of a terminal oxo complex beyond group 8 (figure I.2.3 right) and was obtained upon conversion of $\left[\operatorname{Ir}(\mathrm{Mes})_{3}\right]$ with air or $\mathrm{Me}_{3} \mathrm{NO} .{ }^{[62]}$ However, no further investigations on the reactivity of this compound were reported, due to the difficult and low yield syn- 
thesis of starting $\left[\operatorname{Ir}(\mathrm{Mes})_{3}\right] .{ }^{[63]}$

The formation of four coordinate $\mathrm{Fe}^{\mathrm{IV}}$ nitrido complex was first reported by Peters and coworkers (figure I.2.4 left). ${ }^{[64]}$ Its electronic structure agrees well with considerations of the general frontier-orbital splitting scheme for terminal-nitrido complexes in threefold symmetry, ${ }^{[65]}$ represented by a $\left(d_{x y}, d_{x^{2}-y^{2}}\right)^{2}\left(d_{z^{2}}\right)\left(d_{x z}, d_{y z}\right)$ electronic configuration ${ }^{[64]}$. A shorter $\mathrm{Fe}-\mathrm{N}$ distance of $d^{4} \mathrm{Fe}^{\mathrm{IV}}$ nitride in comparison with the six-coordinate $\mathrm{Fe}^{\mathrm{V}}$ and $\mathrm{Fe}^{\mathrm{VI}}$ nitrido complexes was revealed by X-ray spectroscopy. ${ }^{[66]}$

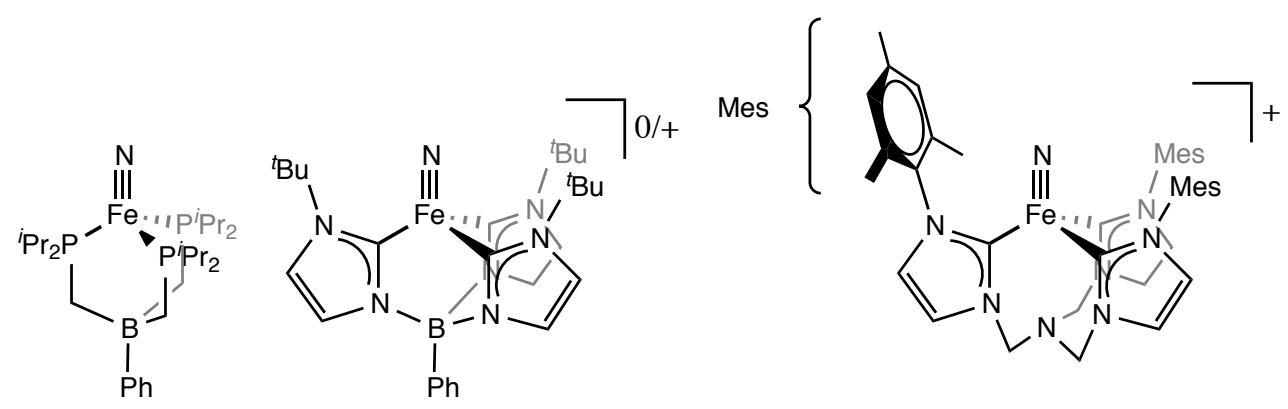

Figure I.2.4.: Nitrido complexes of Fe in three-fold symmetry.

Two independent reports about structural analysis of $\mathrm{Fe}^{\mathrm{IV}}$ nitrido complexes in three fold symmetry by X-ray diffraction were provided by Meyer and Smith, and confirm the short $\mathrm{Fe}-\mathrm{N}$ distance for pseudo-tetrahedral nitrido complexes (figure I.2.4 middle and right). ${ }^{[67,68]}$ Even more remarkable is the structural characterization of a four coordinate $\mathrm{Fe}^{\mathrm{V}}$ nitrido complex. ${ }^{[69]}$ The $\mathrm{Fe}-\mathrm{N}$ distance in this example is shorter than in six coordinate $\mathrm{Fe}^{\mathrm{VI}[58,59]}$, illustrating impressively the importance of the electronic structure in comparison to the metal's formal oxidation state for the bonding situation in nitrido complexes and the high degree in covalency within the M-N moiety. In general, the reported examples of Fe nitrido complexes indicate a higher thermal stability of complexes in three fold than their counterparts in four fold symmetry. ${ }^{[70,71]}$

Energetically low-lying non-bonding $d$-orbitals with respect to the $\mathrm{M}-\mathrm{L}$ bond are also obtained in square-planar coordination geometry. Removal of two apical ligands of an octahedral coordinated complex bearing one multiple bonded ligand results in a squareplanar complex (figure I.2.2 right). Note that the molecular $z$-axis of square-planar complexes is perpendicular to the $\mathrm{ML}_{4}$ plane and therefore localizes the multiple bonded

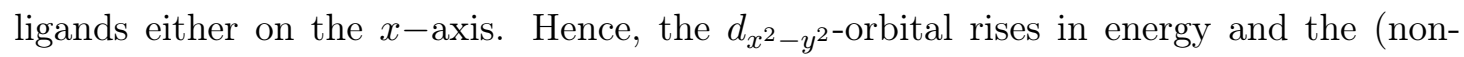

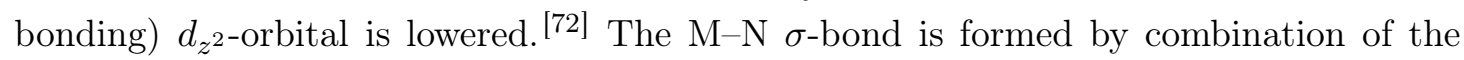


nitrogen $p_{x}$ and the metal $d_{x^{2}-y^{2}}$ orbital and the metals $d_{x z}$ and $d_{x y}$ orbitals form the $\pi$-symmetric MOs by overlapping with the $p_{z}$ and $p_{y}$ orbitals of the multiply bonded ligand, respectively. Occupation of all non-bonding orbitals (with respect to the M-N interaction) affords a total of four electrons and results in a $\left(d_{y z}\right)^{2}\left(d_{z^{2}}\right)^{2}\left(d_{x y}, d_{x z}\right)\left(d_{x^{2}-y^{2}}\right)$ electronic configuration.

In square-planar $d^{4}$ nitrido complexes, the HOMO is mainly represented by the nonbonding $d_{z^{2}}$ orbital, while the LUMO and LUMO +1 are derived from the metal nitride $\pi$ anti-bonding orbital combination. Hence, the frontier orbitals exhibit the same number, energies and symmetry than in octahedral $d^{2}$ systems. This defines an isolobal relationship, suggesting stable nitrido complexes due to the similarities in their electronic structures. ${ }^{[72]}$
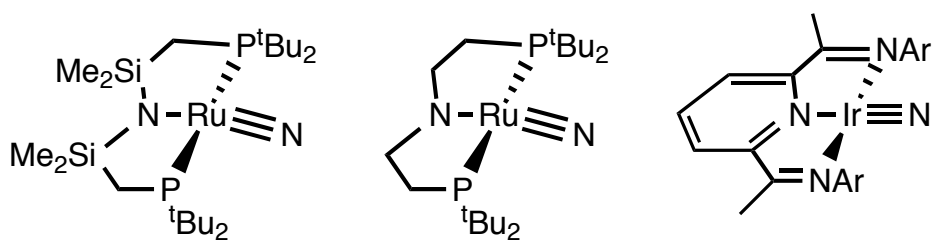

Figure I.2.5.: Examples of stable square-planar terminal nitrido complexes.

Only three examples of square-planar nitrido complexes have been reported, two of $\mathrm{Ru}^{[29,73]}$ and one of $\operatorname{Ir}^{[28]}$ (figure I.2.5), demonstrating the suitability of the square-planar geometry in stabilizing electron rich nitrido complexes of the late transition metals. According to the small number of isolated examples, the reactivity of square-planar nitrido complexes is only sparingly elucidated. Remarkably, all attempts to synthesize isostructural nitrido complexes of $\mathrm{Fe}^{[74]}$ and $\mathrm{Co}^{[75]}$ bearing the $\mathrm{N}\left\{\mathrm{SiMe}_{2} \mathrm{CH}_{2} \mathrm{P}^{t} \mathrm{Bu}_{2}\right\}_{2}$ ligand were hampered by insertion of the nitride into the PNP ligand.

Note that the number of terminal nitrido complexes decrease with increasing group number of the respective transition metal ${ }^{[49]}$ and is in close analogy to the trend observed for related terminal oxo complexes. A strict rule named the 'oxo-wall' was deployed by Ballhausen and Winkler, claiming that there will be no terminal oxo-ligands supported by transition metals beyond group 8 in a tetragonal symmetry. ${ }^{[5]}$ Until today, there are no examples which disprove this rule ${ }^{[76]}$ and with respect to the similarities in bonding and structure between oxo and nitrido complexes ${ }^{[7]}$, it is tempting to build up an analogous 'nitrido wall'. [78] 


\subsection{Reactivity}

\subsubsection{Nucleophilic vs. electrophilic reactivity}

The fundamental reactivity of terminal transition metal nitrides, is determined by the metal, its oxidation state, the coordination geometry and the ancillary ligands. These factors define the energy of the $\pi$-symmetric metal $d$-orbitals and hence localize the LUMO on either the metal or the nitride (figure I.2.6). Nucleophilic attack on the nitrido ligand is expected when the LUMO of the complex is nitrogen centered. ${ }^{[26,46-50]}$ The LUMO and LUMO +1 of closed-shell transition metal nitrido complex are represented by the $\mathrm{M}-\mathrm{N} \pi$ anti-bonding orbital combinations and derive from overlap of a $p$ orbital of the nitride with a $\pi$-symmetric $d$ orbital of the metal (vide supra). Lower energy of the $p$-orbitals of nitrogen than the $d$-orbitals of the metal results in major localization of the HOMO at the nitrogen and result in nucleophilic reactivity of the nitride (case A, figure I.2.6). The other extreme is represented by a metal center with low-lying $\pi$ symmetric $d$-orbitals, resulting in a nitride centered LUMO and hence, in an electrophilic nitrido ligand (case B, figure I.2.6). ${ }^{[46-48]}$ The energy of the $d$ orbitals correlates with their diffusity, which is a function of the atomic radius of the metal. Consequently, the nucleophilic character of nitrido ligands decreases going to top right of the transition metals. ${ }^{[79]}$

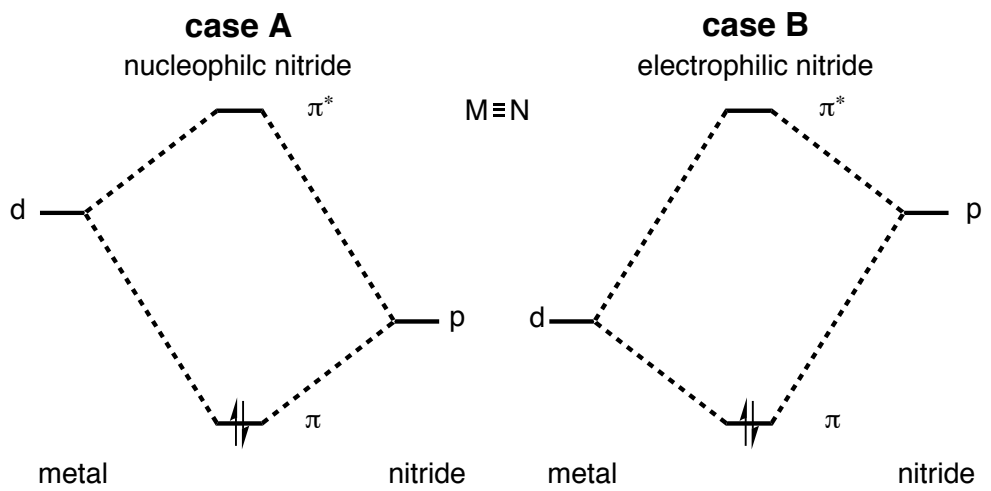

Figure I.2.6.: Simplified $\pi$-interactions of the ligands p-orbitals with the metals $d$-orbitals characterize nucleophilic reactivity of the nitrido ligand (case A) and electrophilic reactivity (case $B$ ). 
Terminal nitrides bound to transition metals lower than group 8 all exhibit nucleophilic behavior. ${ }^{[48]}$ This is demonstrated by conversion with strong electrophiles like methyl triflate ${ }^{[80-83]}$, acid chlorides ${ }^{[84]}$ and trityl tetrafluoroborate ${ }^{[82,83]}$, but also the conversion of nitrides with $\mathrm{CO}$ under formation of isocyanide complexes ${ }^{[29,85-87]}$. Also the formation of adducts with lewis acids such as $\mathrm{AX}_{3}$ or $\mathrm{DX}_{2}(\mathrm{~A}=\mathrm{B}, \mathrm{Al}, \mathrm{Ga}, \mathrm{In} ; \mathrm{D}=\mathrm{Ge}, \mathrm{Sn} ; \mathrm{X}=$ $\mathrm{F}, \mathrm{Cl}, \mathrm{Br}, \mathrm{I})$ is characteristic for nucleophilic reactivity of the nitrido ligand. ${ }^{[88]}$

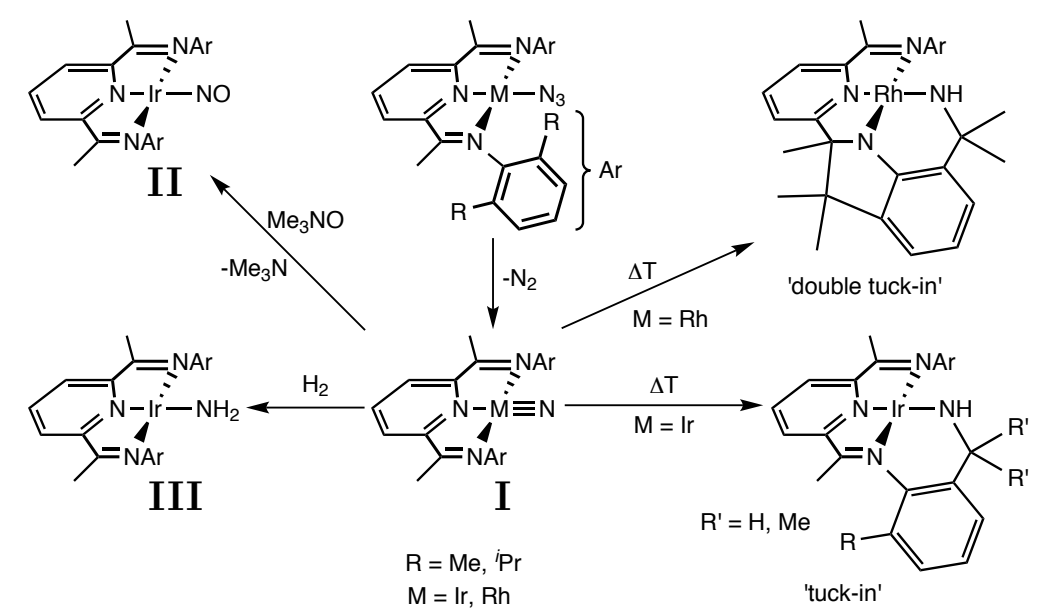

Scheme I.2.2: Synthesis and reactivity of square-planar nitrido complexes of Ir and Rh. Structure $\boldsymbol{I}$ is only proposed in the case of $M=R h$ and fully characterized for $M=I r$.

Beyond group 8, there exists only one example of an isolated terminal nitrido complex. Burger and coworkers reported about thermolysis of $\left[\operatorname{Ir}(\mathrm{PDI})\left(\mathrm{N}_{3}\right)\right]$ (PDI = pyridine-2,6$\operatorname{bis}(N$-(2,6-diisopropylphenyl)ethan-1-imine) ) yielding in the corresponding nitrido complex (I) after release of $\mathrm{N}_{2}$ (scheme I.2.2). Electrophilic behavior of the nitrido moiety was demonstrated by reaction with $\mathrm{Me}_{3} \mathrm{NO}$ under formation of the corresponding nitrosyl complex (II). In addition, the iridium parent amido complex $\left[\operatorname{Ir}(\mathrm{PDI})\left(\mathrm{NH}_{2}\right)\right](\mathbf{I I I})$ was isolated by conversion of $\mathbf{I}$ with $\mathrm{H}_{2}$. This reactivity was attributed to the energetically low lying LUMO, which is localized mainly at the nitrido ligand and inserts into the $\mathrm{H}-\mathrm{H}$ bond. ${ }^{28]}$ However, extended heating of the azide lead to intramolecular $\mathrm{C}-\mathrm{E}(\mathrm{E}=\mathrm{H}$, $\mathrm{Cl}$ ) activation at aromatic substituents on the PDI ligand under formation of 'tuck-in' structures. ${ }^{[89,90]}$ All attempts to isolate the corresponding nitrido complex of rhodium using this ligand manifold resulted in the double tuck-in structure after two successive intramolecular $\mathrm{C}-\mathrm{H}$ activation steps. Based on DFT calculations, a reaction pathway via 
$\mathrm{H}$ atom abstraction of the nitride was proposed for rhodium ${ }^{[89]}$, while the mechanism in the case of iridium most likely proceeds via direct insertion of the nitride into the $\mathrm{C}-\mathrm{E}$ bond. ${ }^{[90]}$

Similar tuck-in structures were obtained for a corresponding Co complex by Chirik and coworkers (scheme I.2.3). Thermolysis of $\left[\mathrm{Co}(\mathrm{PDI})\left(\mathrm{N}_{3}\right)\right]$ (PDI' $=\operatorname{pyridine-2,6-bis}(N$ aryl-1-phenylmethanimine) (IV) results in radical $\mathrm{C}-\mathrm{H}$ activation on the substituents on the aryl group in analogy to the rhodium complex reported by Burger. ${ }^{[91]}$ However, thermolysis of $\mathbf{I V}$ in the presence of $\mathrm{CO}$ yields in the corresponding isocyanato complex. C-N coupling of a nitride with $\mathrm{CO}$ is usually observed for nucleophilic nitrido complexes ${ }^{[29,85,86]}$ and therefore in contrast with the trends within the periodic tables, that expects rather electrophilic reactivity of cobalt nitrido complex. ${ }^{[75]}$ However, C-N coupling of the azide was proposed to proceed prior to the release of $\mathrm{N}_{2}$ for this example and therefore argues against nucleophilic reactivity of a transient Co nitrido complex. ${ }^{[91]}$

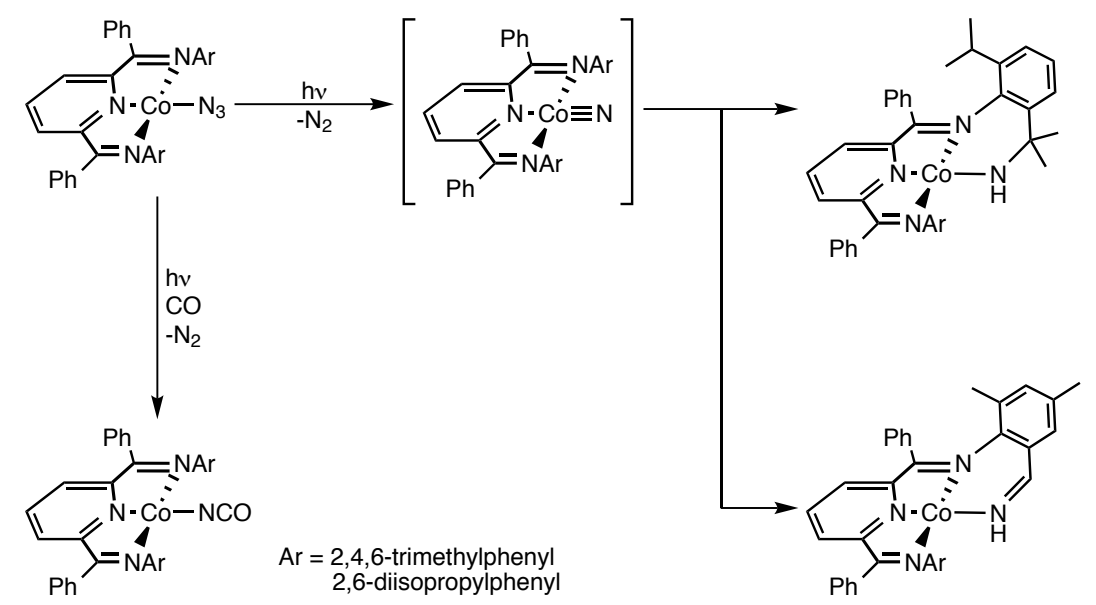

Scheme I.2.3: $C-H$ abstraction of proposed transient $C o$ nitrido complex by Chirik and coworkers and formation of isocyanate complex by photolysis of Co azido complex under CO atmosphere.

Regarding the trends in reactivity of terminal nitrides bound to transition metals of group 9, the electrophilic behavior observed for iridium turns into an open-shell reactivity upon going to the lighter metals. This is in agreement with decrease of ligand-field splitting for the lighter atoms, ${ }^{[92]}$ resulting in smaller energy differences between the singlet and the triplet surface.

Terminal nitrido ligands coordinated to group 8 transition metals are borderline be- 
tween nucleophilic and electrophilic behavior. All nitrido complexes reported for Os are in octahedral coordination geometry with a $d^{2}$ electron count. For these Os ${ }^{\mathrm{VI}}$ nitrides, the reactivity of the nitrido ligand solely depends on the $\pi$-donor strength of the ancillary ligands. ${ }^{48,49]}$ Detailed investigations on $\left[\mathrm{Os}(\mathrm{Tp})(\mathrm{R})_{2}(\mathrm{~N})\right](\mathrm{Tp}=$ hydridotris(pyrazolyl)borate) $(\mathbf{V}: \mathrm{R}=\mathrm{Cl} ; \mathbf{V I}: \mathrm{R}=\mathrm{Ph})$ revealed strong dependence of the reactivity on the nature of the ligand $\mathrm{R}$. Alkylation of the nitride with $\mathrm{PhMgBr}$ is much faster and proceeds with higher conversions in the case of $\mathbf{V}$ than VI. ${ }^{[93]}$ Even more remarkably, reaction of $\mathbf{V}$ with $\mathrm{B}\left(\mathrm{C}_{6} \mathrm{~F}_{5}\right)_{3}$ leads to insertion of the nitride into the $\mathrm{B}-\mathrm{C}$ bond ${ }^{[94]}$, while the formation of a simple Lewis-acid-base adduct is observed for the corresponding reaction of VI (scheme I.2.4). This difference in reactivity is rationalized upon considerations on the energetics of the frontier orbitals. A higher ligand-field splitting is obtained with the stronger donating $\mathrm{Ph}$ group instead of the chloride. Hence, the resulting higher total energy of the LUMO of VI leads to a reduced electrophilic behavior of the nitrido ligand. This picture based on DFT was further supported by higher difference between oxidation and reduction potential of VI than in the case of $\mathbf{V}$ derived by electrochemical investigations. ${ }^{[95]}$

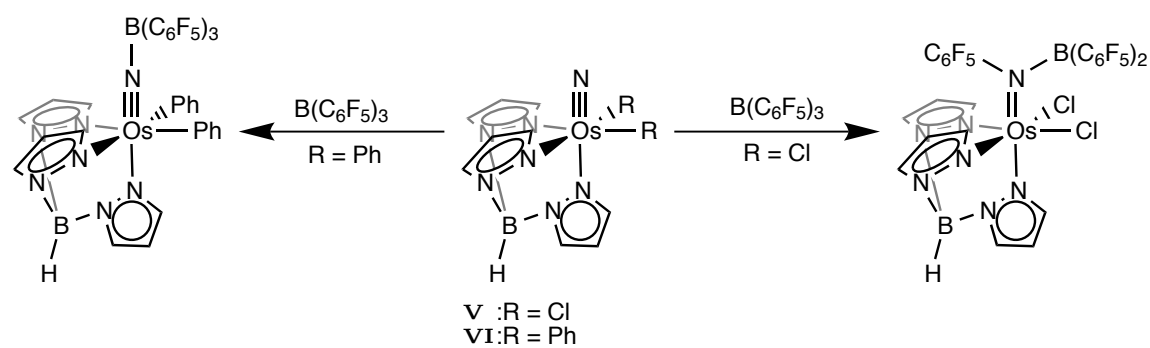

Scheme I.2.4: Dependency of nitride reactivity for $\mathrm{Os}^{V I}$ nitrido complex on the nature of the ancillary ligand $R$. Strong donating Ph group rises the LUMO energy and results in electrophilic nitrido ligand (left) and nucleophilic insertion of the nitride into the $B-C$ bond (right) for $R=C l$.

The reactivity of terminal nitrido complexes of ruthenium depend strongly on the metals oxidation state. Solely electrophilic reactivity is reported for terminal $\mathrm{Ru}^{\mathrm{VI}}$ nitrido complexes and is in agreement with a decrease in energy of the metals $d$ orbitals when going from $\mathrm{Os}^{\mathrm{VI}}$ to $\mathrm{Ru}^{\mathrm{VI}}$. ${ }^{[48,49]}$

Only $\left[\mathrm{Ru}\left\{\mathrm{N}\left(\mathrm{ECH}_{2} \mathrm{P}^{t} \mathrm{Bu}_{2}\right)_{2}\right\}(\mathrm{N})\right]\left(\mathbf{V I I}{ }^{[73]}: \mathrm{E}=\mathrm{SiMe}_{2} ; \mathbf{V I I I}{ }^{[29]}: \mathrm{E}=\mathrm{CH}_{2}\right.$, scheme I.2.5) have been reported as the sole examples of ruthenium nitrides in the oxidation state $+\mathrm{IV}$. 
No discrimination between nucleophilic or open-shell reaction pathway was performed for the conversion of VII with NO under the formation of $\left[\mathrm{Ru}\left\{\mathrm{N}\left(\mathrm{SiMe}_{2} \mathrm{CH}_{2} \mathrm{P}^{t} \mathrm{Bu}_{2}\right)_{2}\right\}(\mathrm{NO})\right]$ and release of $\mathrm{N}_{2} \mathrm{O} .{ }^{[96]}$ However, the conversion of VII with methyl triflate resulted in the corresponding cationic methylimido complex. This reaction was attributed to nucleophilic attack of the nitride at the carbon electrophile. ${ }^{[97]}$ The nucleophilic behavior of the second reported $\mathrm{Ru}^{\mathrm{IV}}$ nitrido complex VIII was demonstrated by selective $\mathrm{C}-\mathrm{N}$ coupling with $\mathrm{CO}$ under formation of $\left[\mathrm{Ru}\left\{\mathrm{N}\left(\mathrm{CH}_{2} \mathrm{CH}_{2} \mathrm{P}^{t} \mathrm{Bu}_{2}\right)_{2}\right\}(\mathrm{NCO})(\mathrm{CO})\right]$. In addition, the full hydrogenation of a terminal metal-nitrido moiety with $\mathrm{H}_{2}$ under release of $\mathrm{NH}_{3}$ could be demonstrated by the conversion of VIII with $\mathrm{H}_{2}$. The resulting ruthenium polyhydrido complex could be converted within two steps into square-planar ruthenium PNP chloro complex IX, which is the starting material for the synthesis of nitride VIII closing a synthetic cycle for the transformation of azide with $\mathrm{H}_{2}$ into ammonia. [29]

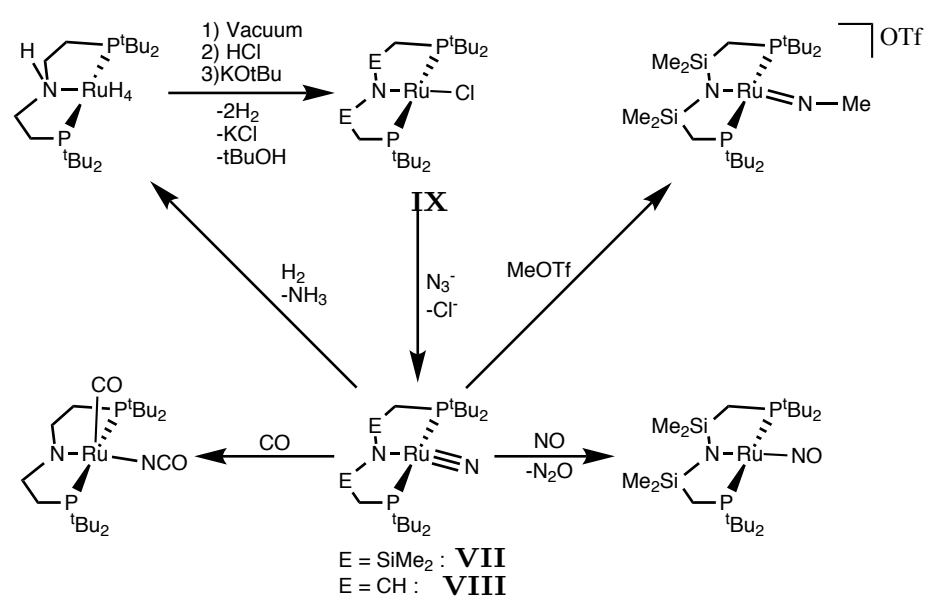

Scheme I.2.5: Reactivity of square-planar nitrido complexes of $R u^{I V}$ (E $=S i M e_{2}$, $\left.\mathrm{CH}_{2}\right)$.

The number of characterized terminal nitrido complexes of Fe is still sizeable. ${ }^{[26,49,50,54]}$ Reactivity studies for octahedral Fe nitrido complexes remain elusive due to their intrinsic low thermal stabilities. Nitride coupling of octahedral $\mathrm{Fe}^{\mathrm{V}}$ nitrido complexes has been described as one major decomposition pathway, ${ }^{[98]}$ but also nitrido-bridged dimeric complexes were found as decomposition products. ${ }^{[55-59,99]}$ Studies of in situ generated octahedral nitrides in the gas phase revealed intramolecular $\mathrm{E}-\mathrm{H}$ activation $(\mathrm{E}=\mathrm{C}, \mathrm{N})$ 


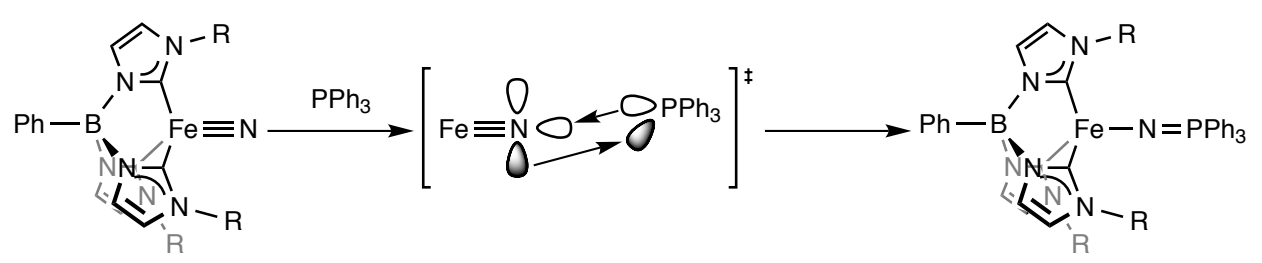

Scheme I.2.6: $N-P$ coupling reaction of $F e^{I V}$ nitrido complex via a dual-nature transition state. Nucleophilic attack of the HOMO of the phosphine at the $\sigma^{*}$-orbital of the $F e \equiv N$ moiety is accompanied by nucleophilic attack of the $\pi$-symmetric HOMO of the nitride at low-lying $P-C \sigma^{*}$-orbital.

or $\mathrm{NH}$ transfer to activated olefines ${ }^{[100]}$, but also electrophilic reactivity was reported for $\mathrm{Fe}^{\mathrm{V}}$ nitrides in solution ${ }^{[70]}$.

$\mathrm{Fe}^{\mathrm{IV}}$ nitrido complexes in three-fold symmetry are more robust than the isolobal octahedral $\mathrm{Fe}^{\mathrm{VI}}$ analogues, as demonstrated by their structural characterization. ${ }^{[67,68]}$ Electrophilic behavior of the nitrido ligand was implied by the formation of phosphoraniminato complexes upon conversion with phosphines (scheme I.2.6). ${ }^{[64,68]}$ Detailed investigations on the mechanism of $\mathrm{N}-\mathrm{P}$ coupling reaction revealed an ambiphilic reactivity of the nitrido ligand. Both nucleophilic attack of the phosphines HOMO at the empty $\pi^{*}$ orbital of the $\mathrm{Fe} \equiv \mathrm{N}$ moiety and the accompanied interaction of the $\sigma$-symmetric lone pair of the nitride with the phosphines LUMO characterizes the dual-nature of the transition state. ${ }^{[69]}$ The ambiphilic character of the nitrido ligand attached to four-coordinate $\mathrm{Fe}^{\mathrm{IV}}$ was further substantiated by $\mathrm{C}-\mathrm{N}$ coupling with $\mathrm{CO}$ and the complete $\mathrm{N}$ atom transfer reaction by conversion with $\mathrm{C} \equiv \mathrm{N}-{ }^{t} \mathrm{Bu}$ towards coordinated carbodiimide (scheme I.2.7). Upon conversion of the latter with benzyl halide, the respective carbodiimide was released under formation of the corresponding $\mathrm{Fe}^{\mathrm{II}}$ halide complex, which closes the synthetic cycle for stoichiometric $\mathrm{N}$ atom transfer. ${ }^{[87]}$

In addition, formation of $\mathrm{NH}_{3}$ could be achieved with $\mathrm{Fe}^{\mathrm{IV}}$ nitrido complexes either by protonation under reductive conditions ${ }^{[64]}$, or by conversion with an $\mathrm{H}$-atom donor such as TEMPO- ${ }^{[101]}$. A radical reaction pathway is suggested for the latter reaction, and is supported by $\mathrm{C}-\mathrm{N}$ coupling of the nitride with the Gomberg radical under formation of Fe ${ }^{\mathrm{III}}$ imido complex. [101]

Furthermore, a four-coordinated $\mathrm{Fe}^{\mathrm{V}}$ nitrido complex was characterized structurally, supporting the higher robustness of Fe-nitrides in three-fold symmetry. In contrast to the $\mathrm{Fe}^{\mathrm{IV}}$ nitrides, conversion of the nitride into $\mathrm{NH}_{3}$ could be achieved using $\mathrm{H}_{2} \mathrm{O}$ and a reducing agent. ${ }^{[69]}$ For the cationic $\mathrm{Fe}^{\mathrm{V}}$ nitrido complex stabilized by the TIMEN ligand 


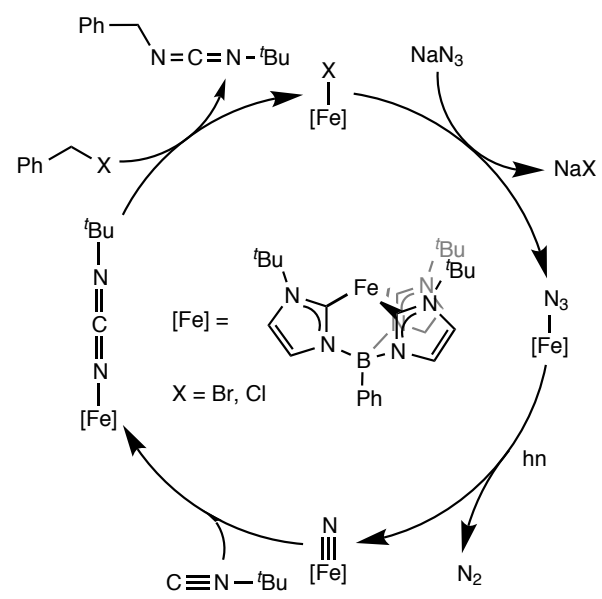

Scheme I.2.7: Synthetic cycle for full $N$ atom transfer of an azide derived Fe nitride under the formation of carbodiimide.

(TIMEN $=$ tris-(2-(3-aryl-imidazo-2-ylidene)ethyl)amine $)$, insertion of the nitride into the Fe-carbene bond was reported, but the mechanism is still not elucidated. [102]

As it was already observed for transition metal complexes of group 9, the reactivity of terminal nitrido complexes of group 8 becomes more dominated by an open-shell character upon ascending within the group.

\subsection{2. $\mathrm{N} \equiv \mathrm{N}$ cleavage and coupling}

The most prominent example for the cleavage of dinitrogen into terminal nitrido complexes was reported by Cummins and coworkers in 1995 (scheme I.1.1 top). ${ }^{[20]}$ The utilization of three coordinated molybdenum triamido complex allowed for the cleavage of $\mathrm{N}_{2}$ into terminal nitrides. The resulting terminal molybdenum nitride forms lewis acid base adducts with a large number of lewis acids, and reacts with strong electrophiles under the formation of nitriles, which could be be further functionalized. ${ }^{88]}$ The nucleophilic reactivity of the Mo nitride also allowed for the formation of nitriles by conversion with acid chlorides. ${ }^{[84]}$ The same reactivity was found for tetrahedral terminal nitrido complexes of $\mathrm{Nb}^{[24]}$ and $\mathrm{W}^{[103]}$ also coordinated by a ligand field consistent of three arylalkylamido ligands. However, the origin of the nitride in the latter cases does not derive from free $\mathrm{N}_{2}$.

More recently, Schrock and coworkers reported the cleavage of $\mathrm{N}_{2}$ into terminal nitrides using a five coordinate Mo precursor resulting in a square-pyramidal complex with the 
nitrido ligand in the apical position. ${ }^{[22]}$ Furthermore, a five coordinate Re complex was reported by Schneider and coworkers to undergo N-N cleavage into a terminal nitrido complex and was further transformed into methyl imido complex upon conversion with methyl triflate. ${ }^{[27]}$

$\mathrm{N} \equiv \mathrm{N}$ bond cleavage by molybdenum is driven by the formation of a very strong $\mathrm{Mo} \equiv \mathrm{N}$ triple bond $(\mathrm{BDE}=155.3 \mathrm{kcal} / \mathrm{mol}) .{ }^{[104]}$ This thermodynamic sink defines the inherent stability of early transition metal nitrides ${ }^{[26]}$, where moderate changes within the (electronic) structure are only induced by conversion with strong electrophiles. ${ }^{[105,106]}$ An increase in reactivity is expected by going to the right in the periodic table. ${ }^{[26,49]}$ Cleavage of the $\mathrm{N} \equiv \mathrm{N}$ triple bond in $\mathrm{N}_{2}$ requires occupation of all anti-bonding MOs, which equals a total amount of six electrons. ${ }^{[64,107,108]}$ Its microscopic reverse ${ }^{[109]}$, the oxidative coupling of two terminal nitrido complexes under formation of $\mathrm{N}_{2}$ allows for mechanistic investigations on this challenging reaction. ${ }^{[10]}$ The discovery of the first $\mathrm{N}_{2}$ coordinated transition-metal complex by Allen and Senoff in $1965^{[111]}$ initiated the search for $\mathrm{N}_{2}$ complexes of other metals ${ }^{[112]}$ under utilization of $\mathrm{NH}_{3}{ }^{[113-116]}, \mathrm{N}_{2} \mathrm{H}_{4}{ }^{[111,117]}, \mathrm{N}_{3}{ }^{-[117]}$ or organic azides ${ }^{[18]}$ besides $\mathrm{N}_{2}$-gas ${ }^{[119]}$ as the nitrogen source. The proposed mechanisms for $\mathrm{N}_{2}$ formation, however, were often lacking experimental support. ${ }^{[112]}$
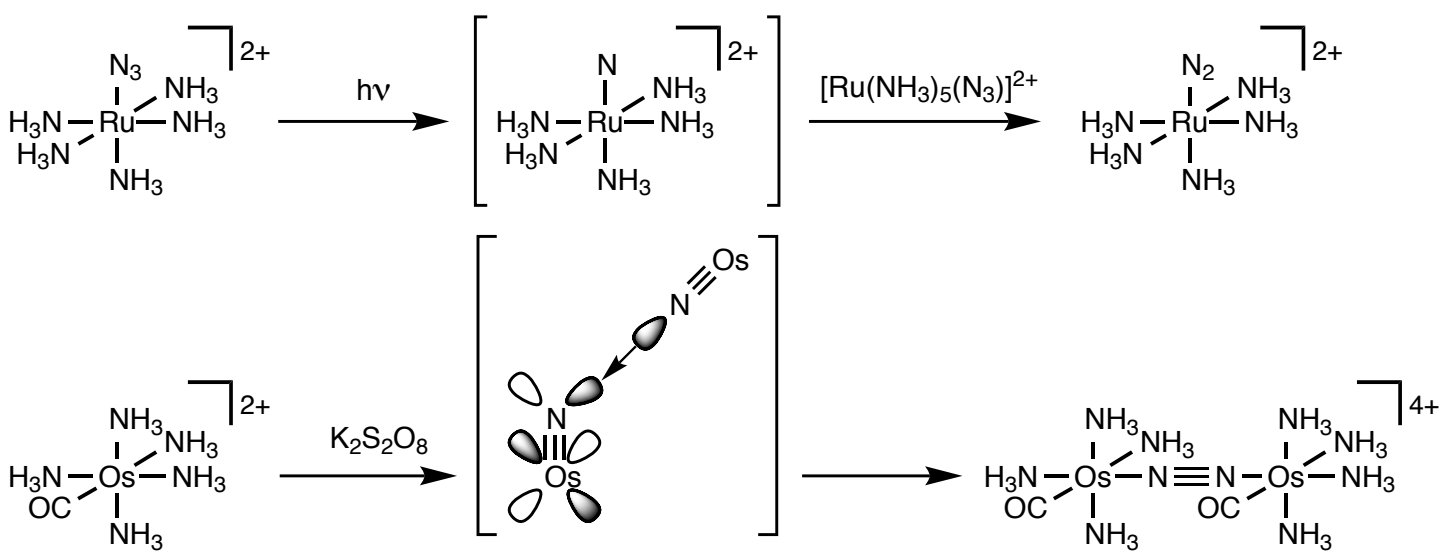

Scheme I.2.8: Proposed coupling intermediates for $N_{2}$ formation reaction. In the case of Ru (top) a metal coordinated nitrene species was postulated as the central intermediate. In contrast, the coupling of two open-shell nitrido complex was suggested in the case of Os (bottom).

Mechanistic studies on the formation of $\mathrm{N}_{2}$ complexes out of protonation or photolysis of $\mathrm{Ru}, \mathrm{Rh}$ and Ir azido complexes were performed by Basolo and coworkers (scheme I.2.8 
top). ${ }^{[120-123]}$ A metal coordinated nitrene $\mathrm{M}-\mathrm{N}$ was proposed as the transient intermediate, which underwent $\mathrm{N}-\mathrm{N}$ coupling reaction. ${ }^{121]}$

The nitrene formalism characterizing an excited-state reactivity was heavily discussed by Taube and coworkers, who attributed the $\mathrm{N}-\mathrm{N}$ coupling to the electronic ground state of the transient intermediate and thus favored the chemical nitrido nomenclature. Experimental support was provided by the formation of a $\mathrm{N}_{2}$ bridged Os dimer by oxidation of monomeric $\left[\mathrm{Os}\left(\mathrm{NH}_{3}\right)_{5} \mathrm{CO}\right]^{2+}$. A transient open-shell nitrido complex $\left[\mathrm{Os}\left(\mathrm{NH}_{3}\right)_{4}(\mathrm{~N}) \mathrm{CO}\right]^{2+}$ was proposed as the reactive intermediate of the $\mathrm{N}-\mathrm{N}$ coupling reaction (scheme I.2.8 bottom). ${ }^{124]}$ In a bent transition state, the HOMO of one nitrido molecule, which represents the Os $\equiv \mathrm{N} \pi$-bonding orbital combination of the metal nitrido entity, is suggested to attacks the Os $\equiv \mathrm{N} \pi^{*}$ anti-bonding orbital combination. The electronic ground-state of the transient open-shell Os nitrido complex is further risen in energy via singly occupation of $\mathrm{Os} \equiv \mathrm{N} \pi^{*}$ orbitals. Hence, the kinetic barrier for $\mathrm{N}-\mathrm{N}$ coupling decreases and therefore accelerates the coupling reaction. ${ }^{[52]}$

$\mathrm{N}_{2}$-bridged dimeric complexes of Os are known in the formal oxidation states of $\mathrm{Os}^{\mathrm{II}} /$ $\mathrm{Os}^{\mathrm{II}}{ }^{[124-127]}$, Os ${ }^{\mathrm{II}} / \mathrm{Os}^{\mathrm{III}}{ }^{[124-130]}$ and $\mathrm{Os}^{\mathrm{III}} / \mathrm{Os}^{\mathrm{III}[124,125,127]}$, either directly formed by nitride coupling or by subsequent oxidation of low valent dimeric complexes. Note that the most stable dimeric complex is represented by both metals in oxidation state $+\mathrm{II},{ }^{[124]}$ and results from nitride coupling of two $\mathrm{Os}^{\mathrm{V}}$ nitrido complexes. ${ }^{[52,110,126,129,131,132]}$ Kinetic studies provided by $\mathrm{N}_{2}$ formation via oxidation of $\left[\mathrm{Os}\left(\mathrm{NH}_{3}\right)_{5}(\mathrm{CO})\right]^{2+}$ suggests an oxidation state of +5 for the coupling intermediate $\left[\mathrm{Os}\left(\mathrm{NH}_{3}\right)_{4}(\mathrm{CO})(\mathrm{N})\right]^{2+} .{ }^{[124]}$ The same intermediate was postulated for oxidative nitride-coupling after photo-excitation of $\mathrm{Os}^{\mathrm{VI}}$ nitrides in the presence of an electron donor under formation of a mixed-valent $\mathrm{N}_{2}$ bridged dimer. This complex results by coupling of photo-excited $\mathrm{Os}^{\mathrm{VI}}$ nitride with a second nitride in the electronic ground-state.

Note that mixed-valent $\mathrm{N}_{2}$ dimers could be formed electrochemically or via chemical reduction of $\mathrm{Os}^{\mathrm{VI}},{ }^{[133]}$ which stresses the importance of the $\mathrm{Os}^{\mathrm{V}}$ nitrido species in all $\mathrm{N}_{2}$ formation reactions. The same intermediate was proposed by Meyer and coworkers for $\mathrm{N}_{2}$ formation upon oxidation of Os polypyridyl coordinated $\mathrm{NH}_{3}$ ligands ${ }^{[130]}$ and electrochemical investigations on $\mathrm{Os}^{\mathrm{VI}}$ nitrides bearing several tridentate ancillary ligands ${ }^{[125-127]}$.

Brown and coworkers studied the thermodynamic and kinetic effects of $\mathrm{N}_{2}$ cleavage. Heating a mixture of a nucleophilic Mo nitride with an electrophilic Os nitride resulted in bimetallic nitrido-coupling under the formation of $\mathrm{N}_{2}$, a $\mu$-nitrido bridged bimetallic dimer and several unidentified side products (scheme I.2.9). Because of the higher 
thermodynamic driving force expected for homocoupling, the observed higher rate for heterocoupling was solely assigned to a kinetic effect. ${ }^{[105]}$

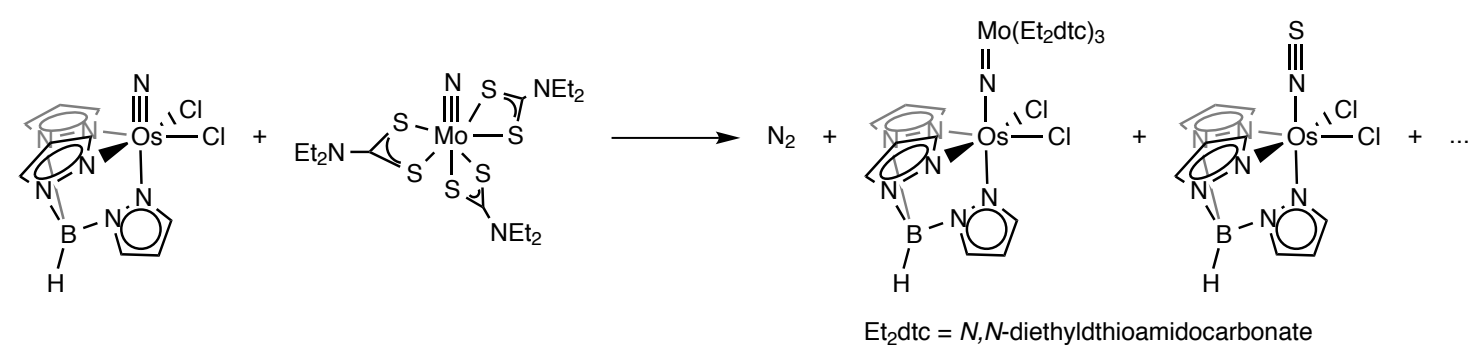

Scheme I.2.9: Bimetallic $N-N$ coupling reaction of a nucleophilic Mo nitride with an electrophilic Os nitride under liberation of $N_{2}$.

$\mathrm{Ru}^{\mathrm{VI}}$ salen complexes have been reported to dimerize via $\mathrm{N}-\mathrm{N}$ coupling reaction in solvents like $N, N$-dimethylformamide or dimethylsulfoxide under release of $\mathrm{N}_{2}$. However, the redox-properties of the $\mathrm{Ru}^{\mathrm{VI}}$ nitrido salen complex are in contrast to one electron reduction prior to nitride coupling. ${ }^{[132]}$

$\mathrm{N}_{2}$ formation was further demonstrated in the case of coordinate $\mathrm{Fe}^{\mathrm{IV}}$ nitride by Peters and coworkers (figure I.2.4 left). ${ }^{[64]}$ The diamagnetic $d^{4}$ nitrido complex is transferred into dimeric high-spin $\mathrm{Fe}^{\mathrm{I}}$ dinitrogen complex ${ }^{[65]}$ and therefore implies a singlet-triplet crossing during the course of the coupling reaction. With respect to the accumulation of open-shell character for intermediates of all reported coupling reactions and the pronounced radical character of nitrido complexes of the $3 d$ transition metals, it is tempting to assign the $\mathrm{N}-\mathrm{N}$ bond forming reactivity to an open-shell reaction, rather then HOMOLUMO interactions. 


\section{Synthesis of late transition metal nitrido complexes}

\subsection{Nitride precursors}

Two fundamental strategies are reported for the synthesis of terminal transition-metal nitrido complexes:

a) Exchange of an ancillary ligand on a complex already bearing the nitride functionality can effect a dramatic change of the nature of the metal complex. ${ }^{[48,50]}$ The success of this approach depends on the stability of the respective starting materials and is often used for high-valent electron poor transition metal nitrides.

b) Formation of a metal nitrogen multiple bond starting from a metal nitrogen single bond. ${ }^{[50]}$ Viable routes are oxidative deprotonation of $\mathrm{NH}_{3}{ }^{[46,127,130,130,134-138]}, \mathrm{N}_{2}$ cleavage $^{[22,108]}$, nitrogen atom transfer reactions ${ }^{[50,64,139]}$ or thermolysis/photolysis of metal azides $^{[28,29,57,59,73,99]}$, but also the $\mathrm{N}-\mathrm{E}$ cleavage $(\mathrm{E}=\mathrm{O}, \mathrm{S}, \mathrm{Si})$ for chalcogenonitrosyl complexes ${ }^{[46,50,134]}$ or silylamines ${ }^{[4,50]}$ has been reported. Besides one example of $\mathrm{Fe}^{\mathrm{IV}[64]}$, all investigations on terminal nitrido complexes of $\mathrm{Fe}, \mathrm{Ru}^{\mathrm{IV}}$ and $\mathrm{Ir}$ were performed starting from low-valent azido complexes. ${ }^{[29,49,50]}$

Complexes bearing azido ligands are usually prepared by salt metathesis starting from the corresponding halides. The transformation of a $\mathrm{M}-\mathrm{N}$ single into a triple bond increases the metals' formal oxidation state of +2 . Hence, nitrido complexes with a $d^{4}$ electron count derive from azide splitting of metal precursors exhibiting a $d^{6}$ electronic configuration. $\mathrm{N}_{3}$ cleavage is facilitated, when the electronic configuration of the metal complex stabilizes the resulting nitrido complex. This is accomplished when the metals' $\pi$-symmetric $d$-orbitals with respect to the $\mathrm{M}-\mathrm{N}$ bond are empty. 


\subsection{Suitable platforms}

A low-spin $d^{6}$ electronic configuration in a square-planar complex was first reported for the $\mathrm{Ru}^{\mathrm{II}}$ chloro complex IX, stabilized by an anionic PNP pincer ligand. ${ }^{[140]}$ In this case, salt-metathesis did not lead to a stable azido complex, but nitride formation was directly observed (scheme I.2.5). ${ }^{[29]}$

A general approach to such platforms is provided by frontier molecular orbital considerations: Octahedral transition metal complexes with six identical solely $\sigma$-donating ligands exhibit two sets of degenerate frontier orbitals $\left(t_{2 g}\right.$ and $\left.e_{g}\right)$ and hence, only two possible spin-states of either fully pairing or unpairing of the electrons (high- and low-spin) are possible (figure I.3.1 left). Formation of a square-planar complex by removal of two apical ligands results in further splitting of the two sets of former degenerated orbitals in a configuration of one set of two degenerate and three non-degenerate frontier orbitals (figure I.3.1 middle). Accordingly, a third intermediate spin state is possible, besides high and low-spin. Upon further increasing of the $\pi$-donor strength of the ligand field, the spin-pairing energy becomes smaller than the energy difference between the two singly occupied orbitals and a low-spin configuration is energetically favored (figure I.3.1 right).

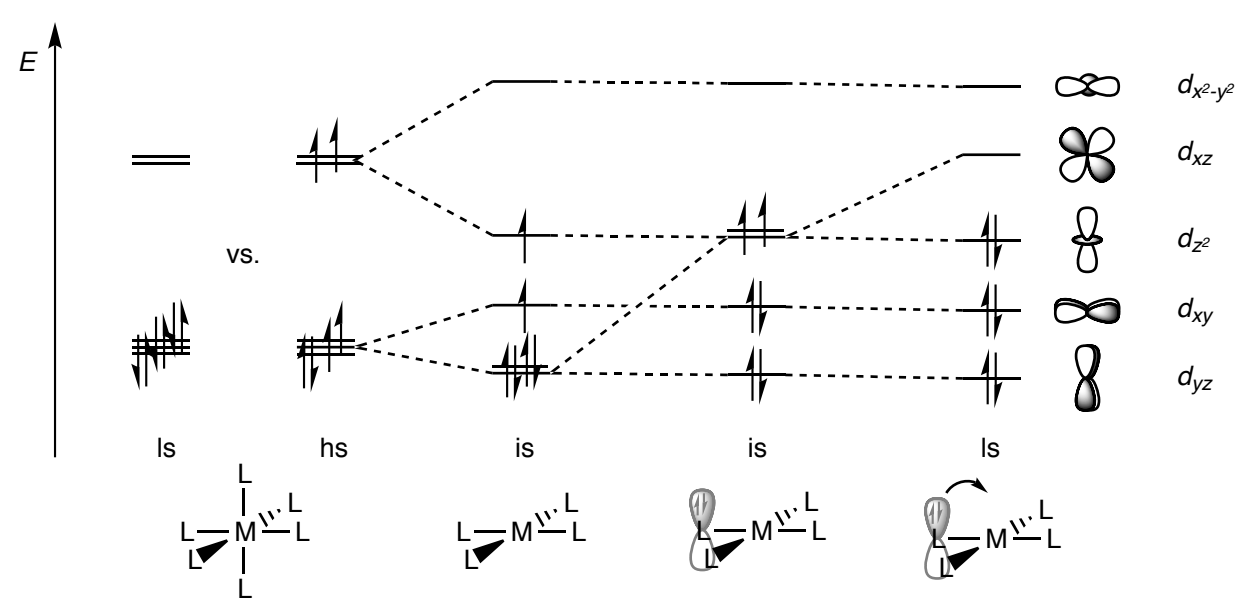

Figure I.3.1.: Frontier orbital splitting scheme for octahedral complexes bearing solely $\sigma$-donating ligands in high-spin (hs) or low-spin (ls) configuration (left). Development of a square-planar ligand splitting by removal of two apical ligands in z-direction allows for the occurrence of an intermediate-spin (is) electronic configuration. Increasing $\pi$-donation rises the energy of the $d_{x z}$ orbital leads to spin-pairing, thus resulting in square-planar complexes in ls configuration. 
The overall spin-state of square-planar complexes depends on the energy difference between the frontier orbitals and the spin-pairing energy. The former is dependent on the $\pi$-donor strength of the ligand field. The spin-pairing energy is a function of the diffuse character of the molecular orbital and correlates with the electronegativity of the metal center. ${ }^{[92,141]}$ Spin-pairing in the set of non-bonding frontier orbitals only occurs in the presence of strong $\pi$-donating ligands like amides. ${ }^{[2]}$ However, interactions between filled metal $d$-orbitals with strong $\pi$-basic amido ligands results in repulsive $p \pi / d \pi$ interactions (vide supra), ${ }^{[43]}$ which lead to degradation of the complexes for example via $\beta$-hydrogen or reductive elimination pathways. ${ }^{[142]}$

The first amido complexes of $\mathrm{Ru}^{\mathrm{II}}$ and $\mathrm{Rh}^{\mathrm{I}}$ were stable enough to be characterized by preventing the presence of $\beta$ hydrogen atoms on the amido ligand, as it is provided by bis(trimethylsilyl)amide. ${ }^{[143]}$ Thermally robust late transition metal amido complexes were isolated by Fryzuk and coworkers using a tridentate chelating pincer ligand consistent of two soft phosphorous and a hard nitrogen atom as donors (figure I.3.2). ${ }^{[144-148]}$

The utilization of a similar ligand manifold allowed for the syn-

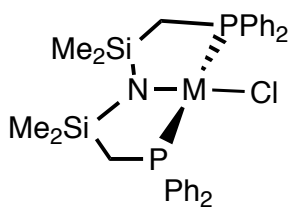

$\mathrm{M}=\mathrm{Ni}, \mathrm{Pd}$

Figure I.3.2.: First stable late transition metal amido complex. thesis of the first example ${ }^{[149]}$ of a square-planar $d^{6}$ complex, namely the $\left[\mathrm{Ru}^{\mathrm{II}}\left\{\mathrm{N}\left(\mathrm{SiMe}_{2} \mathrm{CH}_{2} \mathrm{P}^{t} \mathrm{Bu}_{2}\right)_{2}\right\}(\mathrm{Cl})\right]$ with an intermediate-spin $(\mathrm{S}=1)$ electronic structure. ${ }^{[150]}$ This complex further allowed for the isolation of the first example of a nitrido complex in a (distorted) square-planar coordination geometry with a $d^{4}$ electron count (figure I.2.5 left). [151]

Increasing the $\pi$-basicity of the pincers' amide by substitution of the dimethylsilyl groups against stronger donating alkyl groups (figure I.3.3 right) yields in $\left[\mathrm{Ru}\left\{\mathrm{N}\left(\mathrm{CH}_{2} \mathrm{CH}_{2} \mathrm{P}^{t} \mathrm{Bu}_{2}\right)_{2}\right\}(\mathrm{Cl})\right]$ as the first example of a square-planar complex with a $d^{6}$ electron count in low-spin configuration, ${ }^{[140]}$ and an ideal platform for the synthesis of square-planar Ru nitrido complex (figure I.2.5 middle). ${ }^{[29]}$ However, late transition metal amido complexes bearing $\beta$ hydrogen atoms are prone to imine formation. ${ }^{[140,152]}$ Furthermore, theoretical investigations revealed that the square-planar coordination geometry of this compound is mainly determined by the steric bulk of the tert-butyl substituents on the phosphine groups. ${ }^{[140]}$

A ligand-manifold lacking of $\beta$-hydrogen atoms and $\alpha$-silyl groups is provided by the phenylene bridged PNP pincer ligands, which were employed by Ozerov and coworkers (figure I.3.3 left). ${ }^{[153-156]}$ However, preliminary investigations on the synthesis of the free 


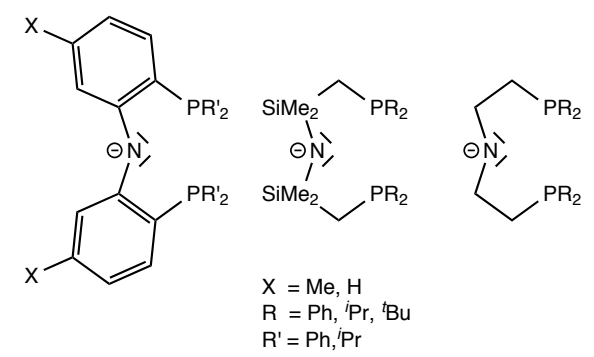

Figure I.3.3.: PNP pincer ligands with increasing $\pi$-donor strength.

ligand with the sterically demanding tert-butyl substituents on the phosphines did not succeed. ${ }^{[157]}$

On the other hand, oxidation of the aliphatic bridged PNP pincer ligand towards enamido $^{[158-160]}$ or dienamido decreases the $\pi$-basicity of the amido donor, however, increases the rigidity and robustness of the ligand framework. These type of ligands should provide a platform, which enables studies on (electronic) structure reactivity relationships of electron-rich late-transition metal nitrido complexes.

In summary, an ideal ligand platform for the synthesis of electron rich transition metal complexes provides a strong $\pi$-donor in combination with steric demanding substituents and avoids the presence of $\beta$-hydrogens or suppression of $\beta$-hydride elimination by exploration chelation. This might be achieved by full dehydrogenation of the ethylene bridged PNP pincer ligand $\mathrm{N}\left(\mathrm{CH}_{2} \mathrm{CH}_{2} \mathrm{P}^{t} \mathrm{Bu}_{2}\right)_{2}$ bearing the large tert-butyl groups.

\subsection{Square-planar iridium(II)}

Recently, Ir ${ }^{\mathrm{I}}$ complex $\left[\mathrm{Ir}(\mathrm{COE})\left\{\mathrm{NH}\left(\mathrm{CH}_{2} \mathrm{CH}_{2} \mathrm{P}^{i} \mathrm{Pr}_{2}\right)_{2}\right\}\right]^{+}(\mathrm{COE}=$ cis-cyclooctene $)$ was reported to undergo reversible intramolecular $\mathrm{C}-\mathrm{H}$ activation at the $\mathrm{COE}$ ligand under formation of an $\mathrm{Ir}^{\mathrm{III}}$ hydride hydrocarbyl complex. ${ }^{[161]}$ Increasing the steric bulk on the PNP pincer ligand by substitution of the iso-propyl groups on phosphorus versus tert-butyl groups was intended to facilitate intermolecular $\mathrm{C}-\mathrm{H}$ activation. The product of the conversion of $\left[\mathrm{Ir}(\mathrm{COE})_{2} \mathrm{Cl}\right]_{2}$ with $\mathrm{NH}\left\{\mathrm{CH}_{2} \mathrm{CH}_{2} \mathrm{P}^{t} \mathrm{Bu}_{2}\right\}_{2}$ (1) was identified as octahedral [ $\left.\mathrm{II}^{\mathrm{III}}\left\{\mathrm{NH}\left(\mathrm{CH}_{2} \mathrm{CH}_{2} \mathrm{P}^{t} \mathrm{Bu}_{2}\right)_{2}\right\}\left(\mathrm{C}_{8} \mathrm{H}_{13}\right)(\mathrm{H})(\mathrm{Cl})\right]$ (2) resulting from intramolecular $\mathrm{C}-\mathrm{H}$ activation of the vinylic $\mathrm{C}-\mathrm{H}$ bond of the $\mathrm{COE}$ ligand (scheme I.3.10). This complex was found to be in a solvent dependent equilibrium with the cationic $\left[\operatorname{Ir}\left\{\mathrm{NH}\left(\mathrm{CH}_{2} \mathrm{CH}_{2} \mathrm{P}^{t} \mathrm{Bu}_{2}\right)_{2}\right\}(\mathrm{COE})\right]^{+}$complex resulting from $\mathrm{C}-\mathrm{H}$ bond formation via re- 
ductive elimination of the cyclooctenyl and hydride ligand. [162]

$\mathbf{2}$ could be oxidized under formation of square-planar $\mathrm{Ir}^{\mathrm{II}}$ complex $\mathbf{3}$ bearing a new fully dehydrogenated anionic PNP pincer ligand (scheme I.3.10). ${ }^{[163]}$

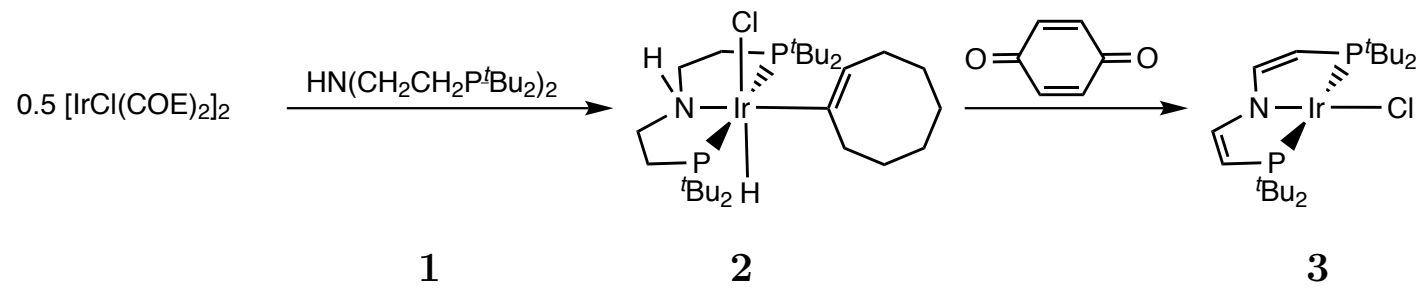

Scheme I.3.10: Synthesis of 3.

The spin doublet electronic ground-state of $\mathbf{3}$ determined by SQUID (superconducting quantum interference device) magnetometry is in agreement with one unpaired electron. The spin density was calculated to be localized in an iridium $d_{x z}$ based molecular orbital assigning $67 \%$ of the spin density on the metal center and was further supported by EPR spectroscopic investigations on 3 . $^{[163]}$

The molecular structure of $\mathbf{3}$ derived by X-ray diffraction studies indicated full dehydrogenation of the PNP pincer backbone and the absence of any hydride ligand could be demonstrated by neutron diffraction studies.

The potential redox non-innocence of amido ligands has attracted considerable interest in recent years. ${ }^{[164-172]}$ For example, radical complexes with related, chelating amido ligands, such as [(COD) $\left.\operatorname{Ir}\left\{\mathrm{N}\left(\mathrm{CHC}_{5} \mathrm{H}_{5} \mathrm{~N}\right)\left(\mathrm{CH}_{2} \mathrm{C}_{5} \mathrm{H}_{5} \mathrm{~N}\right)\right\}\right]$ (COD = 1,5-cyclooctadiene) or $\left[\mathrm{NiCl}\left\{\mathrm{N}\left(\mathrm{C}_{6} \mathrm{MeH}_{3} \mathrm{P}^{i} \mathrm{Pr}_{2}\right) \text { “ }\right\}\right]^{+}$, were reported to exhibit strongly ligand-centered spin densities. ${ }^{[171,172]}$ Thus, resonance structures which describe $\mathbf{3}$ in terms of an iridium(II) amido or iridium(I) aminyl complex provide conceivable alternatives. Mononuclear iridium(II) complexes were frequently postulated as transient reaction intermediates. However, fully characterized examples are more rare than those of rhodium(II). ${ }^{[173-183]}$

One irreversible redox wave, which became quasi-reversible at fast scan rates, and one fully reversible oxidation wave was revealed by electrochemical investigations on $\mathbf{3}$ in DCM solution. Reversible oxidation at $E_{1 / 2}=+0.02 \mathrm{~V}(100 \mathrm{mV} / \mathrm{s})$ even at low scan rates is particularly surprising, as the chemical oxidation of the related complex $\left[\mathrm{Ir}{ }^{\mathrm{II}} \mathrm{Cl}\left\{\mathrm{N}\left(\mathrm{SiMe}_{2} \mathrm{CH}_{2} \mathrm{P}^{t} \mathrm{Bu}_{2}\right\}_{2}\right\}\right]$ resulted in the isolation of subsequent products after $\mathrm{P}^{t} \mathrm{Bu}$ cyclometalation. ${ }^{[183]}$ 
Furthermore, chemical oxidation was also demonstrated by redox titration. The appearance of new signals in the ${ }^{1} \mathrm{H}$ NMR spectrum of an equimolar mixture of $\mathbf{3}$ with $\left[\mathrm{Fe}\left(\mathrm{C}_{5} \mathrm{H}_{5}\right)_{2}\right] \mathrm{PF}_{6}$ indicated the reversible formation of cationic square-planar iridium(III) complex 4. ${ }^{[163]}$ However, isolation of oxidation product 4 was not accomplished so far. 


\section{Scope of this work}

1. The new fully dehydrogenated PNP pincer ligand provides a suitable platform for investigations on multiply bonded ligands at late transition metal complexes. ${ }^{163]}$ Based on the redox properties of fully characterized Ir ${ }^{\mathrm{II}}$ PNP chloro complex $\mathbf{3}$, a redox series of $\mathrm{Ir}^{\mathrm{I}} / \mathrm{Ir}^{\mathrm{II}} / \mathrm{Ir}^{\mathrm{III}}$ complexes in identical coordination environment will be investigated. Furthermore, reactivity studies will be performed to explore (electronic) structure reactivity relationships for the new Ir PNP platform. In particular, the suitability of this platform for small molecule and $\mathrm{C}-\mathrm{H}$ activation will be investigated

2. Nitrido complexes with $d^{n}(\mathrm{n} \geq 4)$ valence-electron counts are particularly rare. ${ }^{[49]}$ Besides the known pseudo-tetrahedral examples (vide supra), ${ }^{[54,184]}$ square-planar $d^{4}$ nitrido complexes should also be accessible, as the isolobal relationship with octahedral $d^{2}$ nitrides suggests that two vacant $d$ orbitals with appropriate symmetry are available for $\mathrm{M} \equiv \mathrm{N} \pi$-bonding. However, isolated examples are restricted to $\mathrm{Ru}$ and Ir nitrido complexes. ${ }^{[28,29,151]}$ The square-planar Ir platform provided by the new fully dehydrogenated PNP pincer ligand will be used for the synthesis of nitrido complexes. Investigations on the electronic structures of these nitride complexes and reactivity studies will be performed.

3. Template synthesis of the new fully dehydrogenated PNP pincer ligand on $\operatorname{Ir}^{[163]}$ and its suitability for the formation of Ir nitrido complexes encourages investigations on reactivity transfer upon Rh. Higher electrophilic reactivity is expected for nitrido complexes of $\mathrm{Rh}$, rather than Ir, expecting a pronounced potential for electrophilic insertion of the nitride into $\mathrm{C}-\mathrm{H}$ bonds. Therefore, investigations on the characterization and reactivity of terminal nitrido complexes of $\mathrm{Rh}$ will be performed . 
4. Oxidative $\mathrm{C}-\mathrm{H}$ amination reactions are usually performed via nitrene insertion of imidoiodinanes. ${ }^{[38]}$ Although intramolecular $\mathrm{C}-\mathrm{H}$ insertion reactions of transient nitrido complexes have been reported, ${ }^{[89-91,185-187]}$ studies on intermolecular $\mathrm{C}-\mathrm{H}$ insertions reactions are limited to gas-phase studies. ${ }^{[100]}$ Based on investigations on Ir nitrido complexes, the interconversion of nitride into imido-, amido and amino complexes will be examined either via successive electron and proton transfer and upon proton coupled electron transfer. The course of the reactions will be rationalized in terms of $\mathrm{N}-\mathrm{H}$ bond dissociation energies.

5. Conversion of electrophilic nitrido complexes with elemental sulfur and selenium to give chalcogenonitrosyl complexes are reported. ${ }^{[188-190]}$ Although the number of thionitrosyl complexes is sizable, yet mostly obtained by other routes, only one example of a selenonitrosyl complex is recorded. ${ }^{[189]}$ Furthermore, formation of nitrosyl complex of reported Ir nitride demonstrated its electrophilic reactivity. ${ }^{[28]}$ The reactivity of Ir nitrido complex stabilized by the fully dehydrogenated PNP pincer ligand will be investigated upon the synthesis of chalcogenonitrosyl complexes. 


\section{Part II.}

Results and Discussion 


\section{Square-planar iridium(I/II/III) platforms}

The results of this chapter were published 2011 under the title: „Square-Planar Iridium(II) and Iridium(III) Amido Complexes Stabilized by a PNP Pincer Ligand“. [163]

\subsection{Iridium(II)}

Full conversion of in situ generated 2 with 1,4-benzoquinone was reported after $16 \mathrm{~h}$ at room temperature, and $\mathbf{3}$ can be isolated after repeated extraction with THF and benzene and subsequent pentane crystallization in analytically pure form. However, the described work-up procedure suffered from only moderate isolated yields of $41 \%$. ${ }^{[163]}$ In contrast, 3 can be isolated from the reaction mixture after stepwise extraction of the reaction product with THF, benzene and pentane. The stoichiometric side product hydroquinone is still remaining within the crude product, but can be removed easily via sublimation $i$. vac. at $60{ }^{\circ} \mathrm{C}$. Hence, an increased yield up to $80 \%$ is provided on this route in sufficient purity. In addition, 3 can further be purified by vacuum sublimation at $100{ }^{\circ} \mathrm{C}$.

\subsection{Iridium(III)}

The electrochemical investigations on $\mathbf{3}$ and the reversibility indicated by the redox titration experiments initiated the synthesis of the primary oxidation product, $[\mathrm{IrCl}\{\mathrm{N}(\mathrm{CHCH}$ $\left.\left.\left.\mathrm{P}^{t} \mathrm{Bu}_{2}\right)_{2}\right\}\right] \mathrm{PF}_{6}$ (4). Conversion of $\mathbf{3}$ with $\mathrm{AgPF}_{6}$ resulted in 4 in $40 \%$ isolated yield (scheme II.1.1). 


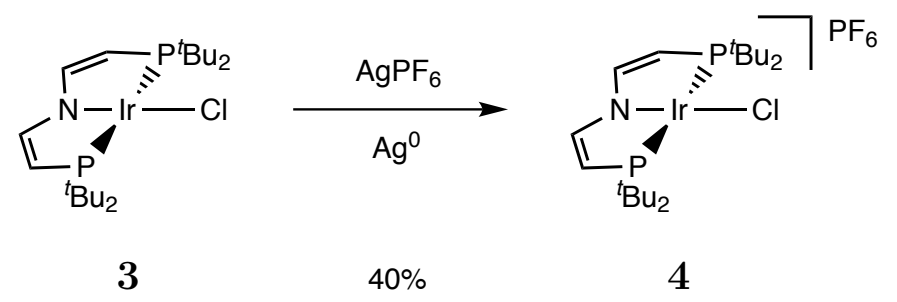

Scheme II.1.1: Oxidation of 3 to cationic 4.

4 is thermally labile, and complete decomposition to several products is observed after $4 \mathrm{~h}$ in solution at room temperature. One singlet in the ${ }^{31} \mathrm{P}$ NMR spectrum and the well resolved mulitplet structures of the signal groups within the ${ }^{1} \mathrm{H}$ NMR spectrum of 4 indicate diamagnetism for $\mathbf{4}$ and $C_{2 V}$ symmetry on the NMR timescale. The diamagnetism of $\mathbf{4}$ is unexpected, as square-planar $d^{6}$ complexes with a formal 14-valence-electron count typically exhibit an electronic intermediate-spin $(\mathrm{S}=1)$ configuration. ${ }^{[140]}$

The molecular structure of $\mathbf{4}$ in the crystalline state confirms the square-planar geometry (figure II.1.1) ${ }^{1}$. The steric bulk of the ${ }^{t} \mathrm{Bu}$ substituents or the planarization of the ligand backbone possibly contribute to the stabilization of square-planar instead of saw-horse coordination, which is generally observed for four-coordinate Ir $^{\mathrm{III}}{ }^{[191]}$ However, DFT calculations predict a square-planar structure for the less sterically encumbered model complex $\left[\operatorname{IrCl}\left\{\mathrm{N}\left(\mathrm{CHCHPMe}_{2}\right)_{2}\right\}\right]^{+}\left(4^{\mathrm{Me}}\right)$, as well (vide infra). The structural parameters of $\mathbf{4}$ and $\mathbf{3}$ in the crystalline state are very similar. As the most striking difference, the Ir1-N1 bond shortens considerably upon oxidation (4 1.922(2), 3 1.986(2)). The comparison with the Ir1-Cl1 bond lengths (4 2.2966(6), $32.3390(7))$ suggests that the Ir-N bond contraction cannot only be attributed to the smaller ionic radius of iridium(III). Furthermore, significant elongation of the pincer backbone N-C bonds are also observed.

The bonding picture was elaborated by electronic structure calculations using DFT methods for the model complexes $\left[\operatorname{IrCl}\left\{\mathrm{N}\left(\mathrm{CHCHPMe}_{2}\right)_{2}\right\}\right]\left(3^{\mathrm{Me}}\right)$ and $[\operatorname{IrCl}\{\mathrm{N}(\mathrm{CHCH}$ $\left.\left.\left.\mathrm{PMe}_{2}\right)_{2}\right\}\right]^{+}\left(4^{\mathrm{Me}}\right){ }^{2} \quad$ The optimized geometries of $3^{\mathrm{Me}}$ (doublet state) and $4^{\mathrm{Me}}$ (singlet state) are in good agreement with the crystallographic results for the respective $\{\operatorname{Ir}(\mathrm{PNP})\}^{0 /+}$ fragments. $4^{\mathrm{Me}}$ (triplet state) is found at higher energies with respect to the singlet state by around $4.1 \mathrm{kcal} / \mathrm{mol}$ (B3LYP) or $9.3 \mathrm{kcal} / \mathrm{mol}(\mathrm{BP})$. Furthermore, the Ir-N bond length is considerably overestimated in the triplet state $\left(\Delta d_{I r-N}=0.08 \AA\right)$.

\footnotetext{
${ }^{1}$ Determination and refinement of the molecular structure of $\mathbf{3}$ in the solid state was performed by Dr. Eberhardt Herdtweck.

${ }^{2}$ DFT calculations were performed by Dr. Marat Khusniyarov.
} 


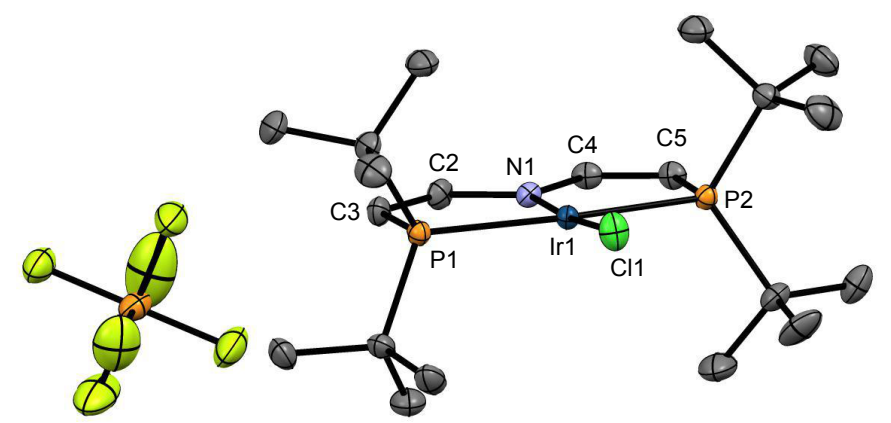

Figure II.1.1.: ORTEP plot of the molecular structure of 4 from single crystal X-ray diffraction (ellipsoids set at $50 \%$ probability, hydrogen atoms and one THF solvent molecule are omitted for clarity). Selected bond lengths $[\AA]$ and angles $\left.I^{\circ}\right]$ : Ir1-Cl1 2.2966(6), Ir1-N1 1.922(2), Ir1-P1 2.3416(6), Ir1-P2 2.3443(6), N1-C2 1.414(3), N1-C4 1.415(3), C2-C3 1.335(3), C4-C5 1.334(3); N1-Ir1-Cl1 174.93(6), P1-Ir1-P2 $167.56(2)$.

The structural trends are easily explained by consideration of the frontier orbitals. The SOMO of $3^{\mathrm{Me}}$ exhibits considerable N-Ir $\pi^{*}$ character and the spin density is mainly localized within the $d_{x z}$-based MO (figure II.1.2 left). Therefore, removal of this electron by oxidation towards $4^{\mathrm{Me}}$ in the singlet state effects reinforcement of the $\mathrm{N}$-Ir $\pi$ - bond (figure II.1.2 right). Therefore, the unusual electronic low-spin configuration of 4 is attributed to strong $\mathrm{N} \rightarrow \mathrm{Ir} \pi$ donation as in the case of $\left[\mathrm{RuCl}\left\{\mathrm{N}\left(\mathrm{CH}_{2} \mathrm{CH}_{2} \mathrm{P}^{t} \mathrm{Bu}_{2}\right)_{2}\right\}\right]$ (s. fig II.1.2). ${ }^{[29,140]}$ While the $\pi$-donor properties of the saturated ligand should be weakened by dehydrogenation to the vinylidene bridged ligand, this effect is counter-balanced by the cationic charge and the change from a $4 d$ to a $5 d$ metal, which should strengthen $\mathrm{M}-\mathrm{N}$ bonding.

The valence-tautomeric description of diamagnetic 4 resulting from ligand centered oxidation of 3 and antiferromagnetic coupling of the ligand with the metal center $\left((\mathrm{L} \cdot) \mathrm{Ir}^{\mathrm{II}}\right)$ cannot be fully excluded. However, a broken-symmetry solution was not found by DFT, and the present experimental results (for example, the Ir-N bond lengths) do not point towards such an electronic structure. Thus, the more simple description $(\mathrm{L})^{-} / \mathrm{Ir}^{\mathrm{III}}(\mathrm{L}=$ $\left.\mathrm{N}\left(\mathrm{CHCHP}^{t} \mathrm{Bu}_{2}\right)_{2}\right)$ is preferred. ${ }^{[192]}$

Isolation of the thermally stable complex $\mathbf{3}$ bearing the new dieneamido ligand was performed via template synthesis. The oxidation towards cationic 4 provides synthetic access to a rare class of coordinatively and electronically unsaturated species. In addi- 

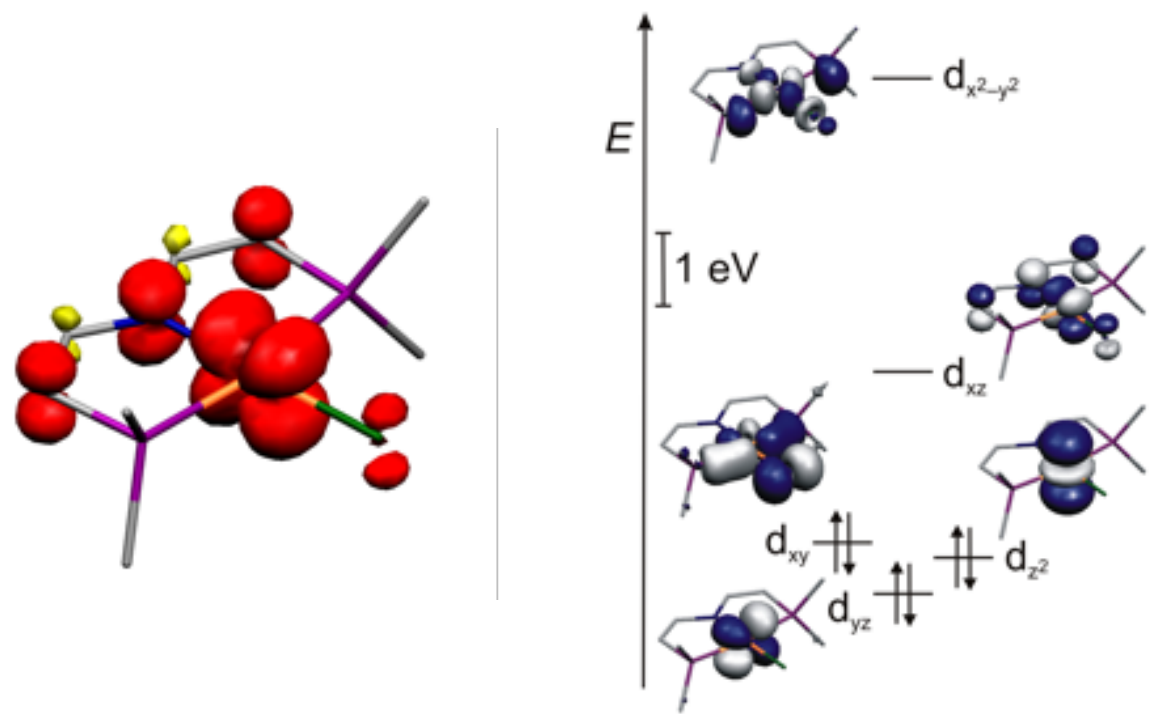

Figure II.1.2.: Spin density map for $3^{M e}$ (left) and metal-centered Kohn-Sham frontier orbitals of $4^{M e}$ in the singlet state (right) from spin-unrestricted ZORA-B3LYP-DFT calculations (the $z$ axis is perpendicular to the [Ir $\left.\left\{\mathrm{N}\left(\mathrm{CHCHPMe}_{2}\right)_{2}\right\}\right]$ plane $)$.

tion, the low-spin electronic configuration provides a suitable platform for investigations on multiple bonded ligands, i.e. the synthesis of iridium nitrido complexes.

\section{3. $\operatorname{Iridium}(\mathrm{I})$}

The results of this chapter were published in 2014 under the title: "/IrCl\{ $\mathrm{N}(\mathrm{CHCH}$ $\left.\left.P^{\mathrm{t}} \mathrm{Bu}{ }_{2}\right)_{2}\right\}^{-}$: a versatile source of the $\operatorname{Ir}^{I}(P N P)$ pincer platform ", ${ }^{[193]}$ and are reproduced by permission of The Royal Society of Chemistry.

The new dieneamido PNP ligand opens up the opportunity to examine an unusual oneelectron reactivity of iridium. In the following chapter, the isolation of anionic $\operatorname{Ir}^{\mathrm{I}}$ PNP chloro complex, which completes an $\mathrm{Ir}^{\mathrm{I}} / \mathrm{Ir}^{\mathrm{II}} / \mathrm{Ir}^{\mathrm{III}}$ redox series will be described.

The electrochemical properties of $\mathbf{3}$ were reevaluated by cyclic voltammetry (CV) in THF (figure II.1.3). The change of solvent showed the $\operatorname{Ir}^{\mathrm{I}} / \mathrm{Ir}^{\mathrm{II}}$ redox couple to be reversible even at low scan rates $(10-400 \mathrm{mV} / \mathrm{s})$ indicating that irreversible reduction of 3 in DCM might be attributed to reoxidation by the solvent. Accordingly, chemical 


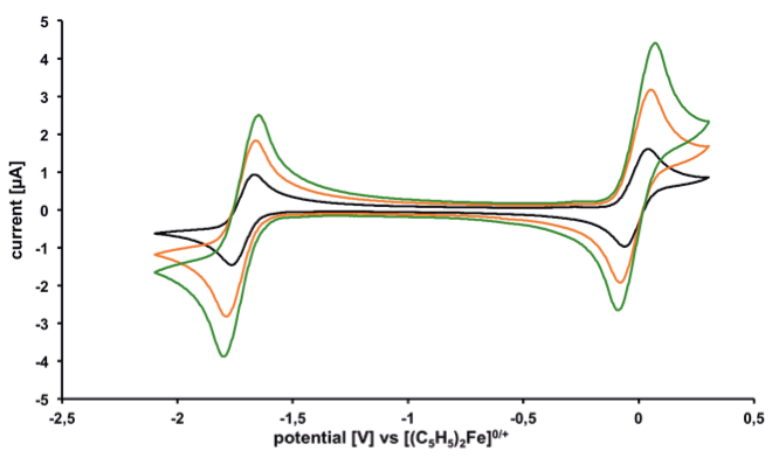

Figure II.1.3.: Cyclic voltammogram of 3 in $T H F\left(0.1 M / n B u_{4} N / P F_{6}\right.$, room temperature, Pt working electrode) at different scan rates (black: $100 \mathrm{mV} / \mathrm{s}$, orange: $400 \mathrm{mV} / \mathrm{s}$, green: $800 \mathrm{mV} / \mathrm{s}$ ).

reduction of 3 with $\mathrm{KC}_{8}$ in THF and in the presence of 15-crown-5 affords the isolation

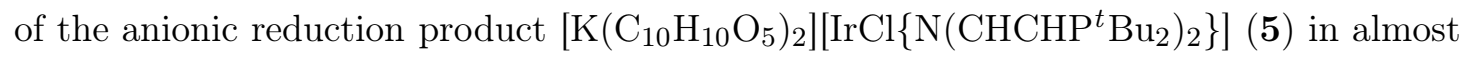
$45 \%$ yield as an orange compound (scheme II.1.2). ${ }^{3}$

The high air sensitivity of 5 is in line with the low oxidation potential $E_{1 / 2}=-1.8 \mathrm{~V}$ obtained by $\mathrm{CV}$. Characterization of $\mathbf{5}$ by NMR spectroscopy at room temperature is in agreement with $C_{2 V}$ symmetry on the NMR timescale. The ${ }^{31} \mathrm{P}$ chemical shift of $\mathbf{5}$ exhibits a remarkable downfield shift by $\Delta \delta=45 \mathrm{ppm}$ as compared with the cationic complex 4 indicating the profound influence of the 2-electron reduction on the paramagnetic screening tensor.

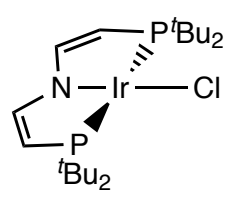

3

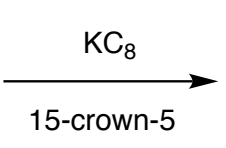

$45 \%$

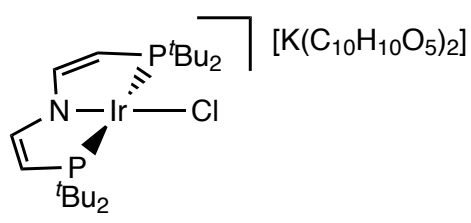

5

Scheme II.1.2: Isolation of anionic 5 by reduction of 3 in the presence of crown ether.

5 was characterized by single crystal X-ray diffraction (figure II.1.4) completing a unique, structurally characterized $\operatorname{Ir}^{\mathrm{I}} / \mathrm{Ir}^{\mathrm{II}} / \mathrm{Ir}^{\mathrm{III}}$ redox series $(\mathbf{5} / \mathbf{3} / \mathbf{4})$ in an identical coordination

\footnotetext{
${ }^{3}$ Isolation and Characterization of $\mathbf{5}$ was performed by M. Sc. Markus Kinauer. ${ }^{[194]}$
} 
environment. ${ }^{4}$ The molecular structure of $\mathbf{5}$ confirms the distorted square-planar ligand arrangement in the solid state. The distortion arises from the pincer bite angle $\left(\mathrm{P} 1-\mathrm{Ir} 1-\mathrm{P} 2: 164.00(3)^{\circ}\right)$, which is the smallest within the series $\left(3: 166.22(2)^{\circ} ; \mathbf{4}\right.$ : $\left.167.56(2)^{\circ}\right)$. This trend seems to be a consequence of the strong dependence of the $\mathrm{Ir}-\mathrm{N}$ bond length on the metal oxidation state, which is transferred via the rigid pincer ligand backbone. In fact, the Ir-N bond becomes considerably shorter on going from $\operatorname{Ir}^{\mathrm{I}}$ $(2.030(2) \AA)$ to $\operatorname{Ir}^{\mathrm{II}}(1.985(2) \AA)$ and $\operatorname{Ir}^{\mathrm{III}}(1.922(2) \AA)$, respectively. ${ }^{[163]}$ This observation can be most easily rationalized with a simple Lewis structure formalism (figure II.1.5).

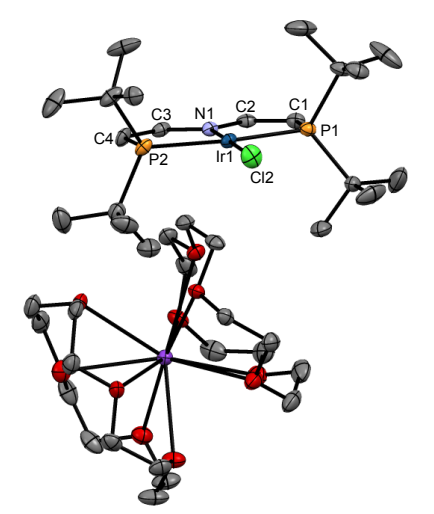

Figure II.1.4.: Molecular structure of 5 in the crystal. Selected bond lengths $[\AA]$ and angles [\% : Ir1-Cl1 2.3995(8), Ir1-N1 2.030(2), Ir1-P1 2.27r9(9), Ir1-P2 2.2760(9), N1-C2 1.370(4), N1-C3 1.369(4), C1-C2 1.349(4), C3-C4 1.350(4); N1-Ir1-Cl1 178.72(7), P1-Ir1-P2 164.00(3).

As $\mathrm{N} \rightarrow \mathrm{Ir} \pi$-donation increases with rising metal oxidation state, the Lewis structure $\mathbf{A}$ is weighed stronger resulting in a shorter Ir- $\mathrm{N}$ distance. Note that the LUMO of $\mathbf{4}$ has a predominantly $\mathrm{Ir}-\mathrm{N} \pi^{*}$-anti-bonding character, therefore exhibiting considerable $\mathrm{Ir}-\mathrm{N}$ double bond character. Upon successive reduction to $\mathbf{3}$ and $\mathbf{5}$, this orbital will be filled with two electrons resulting in no net $\operatorname{Ir}-\mathrm{N} \pi$-bond in $\mathbf{5}$, which provides an MO basis for the observed trend in bond lengths. In turn, the mesomeric Lewis structure $\mathbf{B}$ exhibits a higher weight upon reduction as reflected in the average pincer backbone $\mathrm{N}-\mathrm{C}\left(\mathrm{Ir}^{\mathrm{I}}\right.$ : $\left.1.37 \AA, \mathrm{Ir}^{\mathrm{II}}: 1.39 \AA, \mathrm{Ir}^{\mathrm{III}}: 1.41 \AA\right)$ and $\mathrm{C}-\mathrm{C}\left(\mathrm{Ir}^{\mathrm{I}}: 1.35 \AA, \mathrm{Ir}^{\mathrm{II}}: 1.34 \AA, \mathrm{Ir}^{\mathrm{III}}: 1.33 \AA\right)$ bond lengths. However, the effect is much smaller as compared with the Ir-N bond length. Interestingly, the Ir-P bond lengths (4: $2.34 \AA ;$ 3: $2.32 \AA$ : 5: $2.28 \AA$ ) exhibit the opposite

\footnotetext{
${ }^{4}$ The determination of the molecular structure of $\mathbf{5}$ was performed by M. Sc. Peter Stollberg.
} 


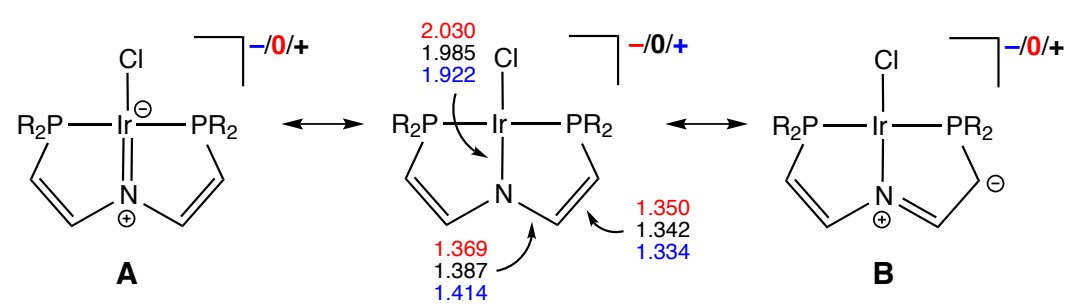

Figure II.1.5.: Selected resonance structures and bond lengths for the redox series 5 (Ir ${ }^{I}$, red), 3 (Ir ${ }^{I I}$, black) and 4 (Ir ${ }^{I I I}$, blue $)$.

trend as expected on a simple consideration of ionic radii, therefore pointing to increased $\mathrm{Ir} \rightarrow \mathrm{P}$ back donation upon reduction.

Hence, the structural parameters within the $\mathrm{Ir}^{\mathrm{I}} / \mathrm{Ir}^{\mathrm{II}} / \mathrm{Ir}^{\mathrm{III}}$ redox series are in agreement with competitive $\pi$-acceptance of the $\mathrm{N}$ lone-pair by the metal center and the vinyl substituents, respectively, subject to the availability of a vacant (4) or half filled (3) metal $d$-orbital with a suitable symmetry. This simple interpretation of the electronic structure explains the stability of this unusual redox series by the divinylamido PNP pincer ligand. The isolation of a $[\mathrm{MCl}(\mathrm{PEP})]^{-}$anion $(\mathrm{M}=\mathrm{Rh}$, Ir; $\mathrm{PEP}=$ anionic pincer ligand $)$ is unprecedented in pincer chemistry. One related compound, $\left.\left[\operatorname{Ir}^{\mathrm{I}}(\operatorname{dippe})_{2}\right]^{+}\left[\operatorname{IrCl}_{2}{ }^{\mathrm{I}} \text { (dippe }\right)_{2}\right]^{-}$ (dippe $\left.={ }^{i} \mathrm{Pr}_{2} \mathrm{P}\left(\mathrm{CH}_{2}\right)_{2} \mathrm{P}^{i} \mathrm{Pr}_{2}\right)$, was previously reported as a coordinatively relatively labile intermediate leading to $[\operatorname{Ir}(\mu-\mathrm{Cl})(\text { dippe })]_{2}$ and $\left[\operatorname{Ir}(\text { dippe })_{2}\right] \mathrm{Cl}^{\left[{ }^{[195]}\right.}\left[\operatorname{Ir}(\mathrm{OTf})_{2} \text { (dfepe) }\right]^{-}$ (dfepe $\left.=\left(\mathrm{C}_{2} \mathrm{~F}_{5}\right)_{2} \mathrm{P}\left(\mathrm{CH}_{2}\right)_{2} \mathrm{P}\left(\mathrm{C}_{2} \mathrm{~F}_{5}\right)_{2}\right)$ with a highly fluorinated phosphine ligand was also reported, pointing to stabilization by $\pi$-acidic ligands. ${ }^{[196]}$ Accordingly, the dihalodicarbonyl anions of iridium are known for a long time and play an important role in catalytic carbonylation reactions. ${ }^{[197]}$ In this case, the combination of steric protection and electronic flexibility provided by the pincer ligand effectively stabilizes the unusually electron rich anionic complex and the complete $\operatorname{Ir}^{\mathrm{I}} / \mathrm{Ir}^{\mathrm{II}} / \mathrm{Ir}^{\mathrm{III}}$ redox series.

\subsection{Reactivity of the iridium(I) PNP chloride}

\subsubsection{Iridium(I) carbonyl complex}

The electronic properties of the Ir PNP pincer platform were further probed by the preparation of the $\operatorname{Ir}^{\mathrm{I}}$ carbonyl complex (scheme II.1.3) $\left[\mathrm{Ir}(\mathrm{CO})\left\{\mathrm{N}\left(\mathrm{CHCHP}^{t} \mathrm{Bu}_{2}\right)_{2}\right\}\right](\mathbf{6}) . \mathbf{6}$ can be isolated from direct reduction of $\mathbf{3}$ with $\mathrm{KC}_{8}$ under an atmosphere of $\mathrm{CO}$, demon- 


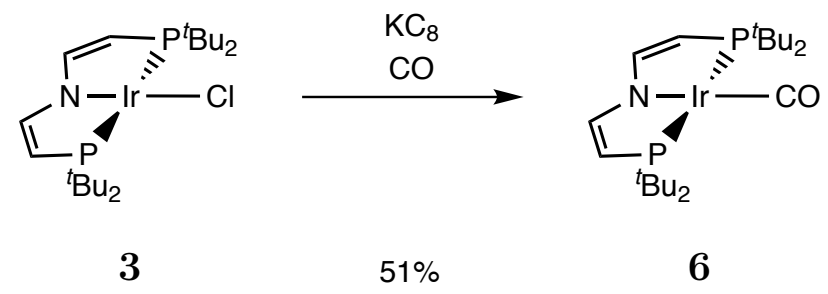

Scheme II.1.3: Synthesis of iridium(I) carbonyl complex 6 by in situ reduction of 3 under an atmosphere of $\mathrm{CO}$.

strating the suitability of the chloride as a leaving group. Interestingly, $\mathbf{5}$ is stable in solution in the absence of $\mathrm{CO}$, yet readily reacts with $\mathrm{CO}$ as well, to give $\mathbf{6}$. Hence, this observation supports an associative $\mathrm{Cl} v$ s. $\mathrm{CO}$ substitution mechanism after reduction of 3 .

The solution NMR data of 6 are in agreement with $C_{2 V}$ symmetry. The molecular structure in the solid state (figure II.1.6) was also derived by single-crystal X-ray diffraction, confirming the distorted square-planar coordination geometry around the metal center with a P1-Ir1-P1 bite angle $\left(163.37(2)^{\circ}\right)$ similar to that in 5. Accordingly, the Ir-N distance $(2.061(2) \AA)$ is slightly longer as in the case of parent $5(2.030(2) \AA)$ as a consequence of the higher $\mathrm{CO} v s$. $\mathrm{Cl}$ trans-influence, again emphasizing the relationship of the pincer bite angle and the Ir- $\mathrm{N}$ distance within this rigid framework. ${ }^{5}$

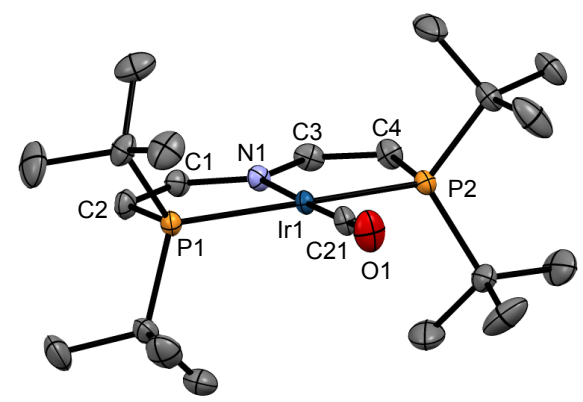

Figure II.1.6.: Molecular structure of $\boldsymbol{6}$ in the crystal. Selected bond lengths [A] and angles $\Gamma^{\circ}$ ]: Ir1-C21 1.829(2), Ir1-N1 2.061(2), Ir1-P1 2.3137(6), Ir1-P2 2.3080(6), N1-C1 1.367(3), N1-C3 1.371(3), C1-C2 1.347(3), C3-C4 1.351(3), O1-C21 1.153(3); N1-Ir1-C21 178.20(9), P1-Ir1-P2 163.37(2).

\footnotetext{
${ }^{5}$ X-Ray diffraction was performed by Dr. Frank W. Heinemann.
} 
The CO stretching vibration of 6 can be assigned to a signal at $1937 \mathrm{~cm}^{-1}$ in the IR spectrum. This value compares well with the $\mathrm{CO}$ band of $\left[\operatorname{Ir}(\mathrm{CO}) \mathrm{Cl}\left(\mathrm{P}^{i} \mathrm{Pr}_{3}\right)_{2}\right]\left(1939 \mathrm{~cm}^{-1}\right)^{[198]}$ or with some corresponding disilylamido ([Ir(CO) $\left.\left.\left(\mathrm{N}\left\{\mathrm{SiMe}_{2} \mathrm{CH}_{2} \mathrm{P}^{t} \mathrm{Bu}_{2}\right\}_{2}\right)\right]: 1930 \mathrm{~cm}^{-1}\right)^{[148]}$ and diarylamido $\left(\left[\mathrm{Ir}(\mathrm{CO})\left(\mathrm{N}\left\{\mathrm{C}_{6} \mathrm{H}_{3} \mathrm{CH}_{3} \mathrm{P}^{i} \mathrm{Pr}_{2}\right\}_{2}\right)\right]: 1930 \mathrm{~cm}^{-1}\right)^{[199]} \mathrm{PNP}$ pincer complexes.

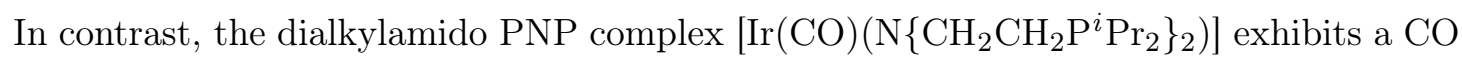
band at considerably lower wavenumber $\left(1908 \mathrm{~cm}^{-1}\right),{ }^{[161]}$ emphasizing reduced $\mathrm{L} \rightarrow \mathrm{M}$ electron donation by the divinylamido ligand. $\sigma$ - and $\pi$-bonding effects cannot be separated merely from the $\mathrm{CO}$ stretching vibration. However, the conjugation of the azallylic $\mathrm{C}=\mathrm{C}$ double bonds with the N-lone pair, which is indicated by the trends within the pincer ligand backbone bond lengths of the $\mathrm{Ir}^{\mathrm{I}} / \mathrm{Ir}^{\mathrm{II}} / \mathrm{Ir}^{\mathrm{III}}$ redox series (vide supra), suggests that reduced $\mathrm{L} \rightarrow \mathrm{M} \pi$-donation of the divinyl- $v s$. the dialkylamido ligand probably constitutes a significant contribution to the higher CO stretching vibration in 6 .

Clean reduction of $\mathbf{3}$ is also accomplished by reaction with $n \mathrm{BuLi}$. Monitoring this reaction by ${ }^{31} \mathrm{P}$ NMR spectroscopy reveals the quantitative formation of a diamagnetic compound at $56.5 \mathrm{ppm}$ (THF), which was assigned to $\mathrm{Li}\left[\operatorname{IrCl}\left(\mathrm{N}\left\{\mathrm{CHCHP}^{t} \mathrm{Bu}_{2}\right\}_{2}\right)\right]$ by comparison with 5 (55.5 ppm). These solutions were only stable below $-20{ }^{\circ} \mathrm{C}$, probably owing to $\mathrm{LiCl}$ elimination. However, the $\mathrm{Ir}(\mathrm{PNP})$ fragment could be trapped by thawing a frozen solution under a $\mathrm{N}_{2}$ atmosphere $(1$ bar $)$ giving $\left[\mathrm{Ir}\left\{\mathrm{N}\left(\mathrm{CHCHP}^{t} \mathrm{Bu}_{2}\right)_{2}\right\}\left(\mathrm{N}_{2}\right)\right](\mathbf{7})^{6}$ in around $60 \%$ spectroscopic yield by ${ }^{31} \mathrm{P}$ NMR spectroscopy (scheme II.1.4).

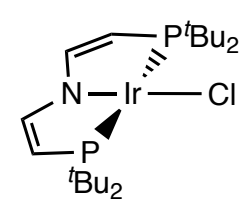

3

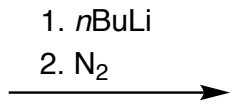

$60 \%$

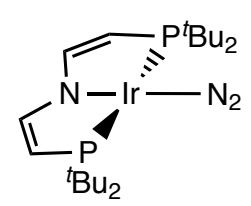

7

Scheme II.1.4: Formation of dinitrogen complex $\boldsymbol{7}$ by in situ reduction of 3 under an atmosphere of $N_{2}$.

Hence, the observation of dinitrogen complex 7 suggests that $\mathrm{C}-\mathrm{H}$ activation reactions should be carried out under argon to avoid inhibition by $\mathrm{N}_{2}$ binding to the $\operatorname{Ir}(\mathrm{PNP})$ fragment. This observation was also reported for the $\operatorname{Ir}\left(\mathrm{C}_{6} \mathrm{H}_{3}\left\{\mathrm{CH}_{2} \mathrm{P}^{t} \mathrm{Bu}_{2}\right\}_{2}\right)$ platform. ${ }^{[201,202]}$

\footnotetext{
${ }^{6}$ Characterization of $\operatorname{Ir}^{\mathrm{I}} \mathrm{N}_{2}$ complex 7 will be provided in chapter $2^{[200]}$
} 


\subsubsection{Oxidative addition to iridium(I) PNP chloride}

Besides substitution for CO, chloride dissociation can also be utilized as the source of $\operatorname{Ir}\left(\mathrm{N}\left\{\mathrm{CHCHP}^{t} \mathrm{Bu}_{2}\right\}_{2}\right)$, which undergoes $\mathrm{C}-\mathrm{H}$ oxidative addition. Reduction of $\mathbf{3}$ with $\mathrm{Na} / \mathrm{Hg}$ in benzene results in facile formation of the red $\mathrm{Ir}^{\mathrm{III}}$ hydride phenyl complex 8 in almost quantitative spectroscopic $\left({ }^{31} \mathrm{P}\right.$ NMR) yield (scheme II.1.5). Isolation of the highly lipophilic compound by crystallization from pentane resulted in isolated yields just below $50 \%$.

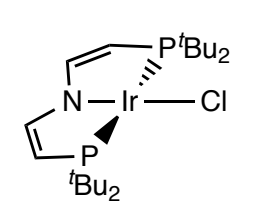

3

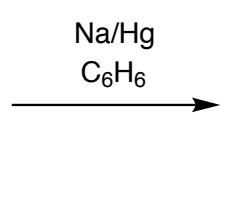

$41 \%$

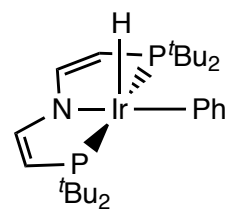

8

Scheme II.1.5: $C-H$ oxidative addition of benzene after in situ reduction of 3.

The addition of benzene to Ir $^{\mathrm{I}}$ PNP was confirmed by NMR spectroscopy and elemental analysis. The chemical shift of the hydride ligand $(\delta=-46.5 \mathrm{ppm})$ suggests a vacant coordination site in trans-position, and hence a square pyramidal coordination geometry around the metal. The three phenyl ${ }^{1} \mathrm{H}$ and four ${ }^{13} \mathrm{C}$ NMR signals at room temperature, respectively, indicate rapid rotation around the $\mathrm{Ir}-\mathrm{C}$ bond on the NMR timescale.

The structural assignments from solution NMR spectroscopy were confirmed by single crystal X-ray diffraction. ${ }^{7}$ In the solid state (figure II.1.7), the five-coordinate metal atom exhibits a square-pyramidal coordination geometry with the hydride ligand in apical position and an almost linear N-Ir-phenyl bond angle $\left(179.8\left(39^{\circ}\right)\right.$. The Ir1-N1 bond $(2.109(6) \AA)$ is particularly long due to the strong trans-ligand $\mathrm{C}_{6} \mathrm{H}_{5}$ also resulting in a smaller P1-Ir1-P2 pincer bite angle $\left(162.58(7)^{\circ}\right)$.

The $\mathrm{C}-\mathrm{H}$ oxidative addition most likely proceeds via three-coordinate $[\operatorname{Ir}(\mathrm{N}\{\mathrm{CHCHP}$ $\left.\left.{ }^{t} \mathrm{Bu}_{2}\right\}_{2}\right)$ ] after reduction of $\mathbf{3}$ and $\mathrm{NaCl}$ elimination in the absence of stabilizing crownether. Such three-coordinate $d^{8}$ intermediates are prone to $\mathrm{C}-\mathrm{H}$ oxidative addition via an intermediate $\mathrm{C}-\mathrm{H} \sigma$-complex. ${ }^{[203]}$ However, halides as leaving groups were not previously reported for their formation.

\footnotetext{
${ }^{7}$ Structural characterization of $\mathbf{8}$ was performed by Dr. Christian Würtele.
} 


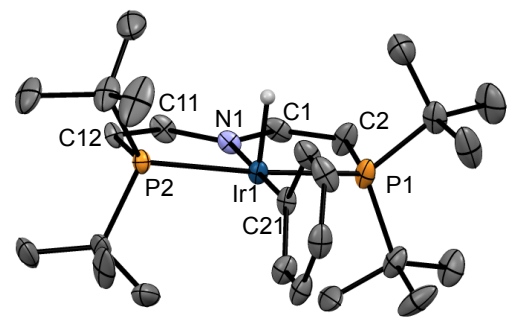

Figure II.1.7.: Molecular structure of $\boldsymbol{8}$ in the crystal. Selected bond lengths [A] and angles [ ${ }^{\circ}$ : Ir1-C21 2.080(7), Ir1-N1 2.109(6), Ir1-P1 2.337(2), Ir1-P2 2.3276(19), N1-C1 1.348(10), N1-C11 1.357(10), C1-C2 1.362(12), C11-C12 1.352(10); N1-Ir1-C21 179.8(3), P1-Ir1-P2 162.58(7).

Besides nucleophilic $\mathrm{C}-\mathrm{H}$ activation, attack of C-electrophiles also provides access to $\mathrm{Ir}^{\mathrm{III}}$ hydrocarbyl complexes. The reaction of $\mathbf{5}$ with MeOTf yields the Ir ${ }^{\mathrm{III}}$ methyl complex $\left[\mathrm{IrCl}\left(\mathrm{CH}_{3}\left(\mathrm{~N}\left\{\mathrm{CHCHP}^{t} \mathrm{Bu}_{2}\right\}_{2}\right)\right](\mathbf{9})\right.$ in around $90 \%$ yield (scheme II.1.6). ${ }^{8}$ Spectroscopic characterization of $\mathbf{9}$ is in agreement with $C_{S}$ symmetry on the NMR timescale. The methyl ligand was assigned to signals at $2.08\left({ }^{1} \mathrm{H} \mathrm{NMR}\right)$ and $-27.1 \mathrm{ppm}\left({ }^{13} \mathrm{C} \mathrm{NMR}\right)$, respectively, both exhibiting triplet multiplicity due to coupling with the ${ }^{31} \mathrm{P}$ nuclei, unequivocally indicating formation of an Ir ${ }^{\mathrm{III}}$ methyl complex. In comparison, the dialkylamido complex $\left[\operatorname{Ir}\left(\mathrm{PMe}_{3}\right)\left(\mathrm{N}\left\{\mathrm{CH}_{2} \mathrm{CH}_{2} \mathrm{P}^{i} \mathrm{Pr}_{2}\right\}_{2}\right)\right]$ is selectively methylated with MeOTf at the ligand nitrogen atom to form the $\operatorname{Ir}^{\mathrm{I}}$ complex $\left[\mathrm{Ir}\left(\mathrm{PMe}_{3}\right)\left(\mathrm{MeN}\left\{\mathrm{CH}_{2} \mathrm{CH}_{2} \mathrm{P}^{i} \mathrm{Pr}_{2}\right\}_{2}\right)\right]$ OTf. ${ }^{[204]}$ Such a ligand centered nucleophilic reactivity was similarly observed for $\mathrm{Pd}^{\mathrm{II}}$ dialkylamido complexes with MeOTf. ${ }^{[205,206]}$ Also, the five-coordinate alkylvinylamido $\mathrm{Ru}^{\mathrm{II}}$ complex $\left[\mathrm{RuH}\left(\mathrm{PMe}_{3}\right)\left\{\mathrm{N}\left(\mathrm{CHCHP}^{i} \mathrm{Pr}_{2}\right\}\left\{\mathrm{CH}_{2} \mathrm{CH}_{2} \mathrm{P}^{i} \mathrm{Pr}_{2}\right\}\right)\right]$ exhibits exclusive ligand methylation upon reaction with MeOTf. ${ }^{207]}$ In this context, the high selectivity of metal alkylation in the case of $\mathbf{5}$ is remarkable and emphasizes the rigid, pincer-type behavior of the chelating ligand.

${ }^{8}$ Isolation and characterization of $\mathbf{9}$ was performed by M. Sc. Markus Kinauer. ${ }^{[194]}$ 


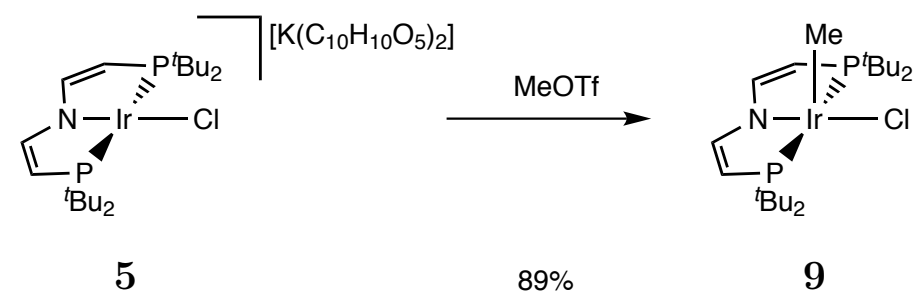

Scheme II.1.6: Electrophilic attack of methyl triflate on $\mathbf{5}$ under formation of iridium methyl complex $\mathbf{9}$.

\subsection{3. $\mathrm{O}_{2}$ activation}

The activation of dioxygen with iridium complexes has been the subject of several studies, e.g. in the context of alkene oxygenation. ${ }^{[208-210]}$ With phosphine pincer ligands, the formation of $\mathrm{Ir}^{\mathrm{III}}$ peroxo complexes, such as $\left[\mathrm{Ir}\left(\mathrm{O}_{2}\right)\left(\mathrm{N}\left\{\mathrm{SiMe}_{2} \mathrm{CH}_{2} \mathrm{P}^{t} \mathrm{Bu}_{2}\right\}_{2}\right)\right]$ or $\left[\operatorname{Ir}\left(\mathrm{O}_{2}\left(\mathrm{C}_{6} \mathrm{H}_{3}\left\{\mathrm{CH}_{2} \mathrm{P}^{t} \mathrm{Bu}_{2}\right\}_{2}\right)\right]\right.$, was reported upon hydrocarbon reductive elimination from $\mathrm{Ir}^{\mathrm{III}}$ under $\mathrm{O}_{2} \cdot{ }^{[183,210]}$ However, to the best of our knowledge, the only crystallographically characterized iridium pincer mono- $\mathrm{O}_{2}$ adduct is the 'POCOP'-complex $\left[\operatorname{Ir}\left(\mathrm{O}_{2}\right)\left\{\mathrm{C}_{6} \mathrm{H}_{3}\right.\right.$-2,6$\left.\left.\left(\mathrm{PPR}_{2}^{F}\right)_{2}\right\}\right]\left(\mathrm{R}^{F}=\mathrm{C}_{6} \mathrm{H}_{2}-2,4,6-\left(\mathrm{CF}_{3}\right)_{3}\right)$ which was synthesized in the solid state and was in turn not spectroscopically examined. ${ }^{[211]}$ Hence, a full set of spectroscopic and structural data for this class of compounds is surprisingly not available.

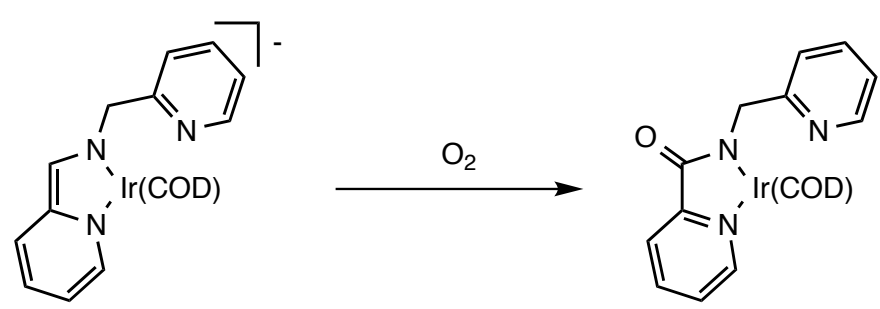

Scheme II.1.7: Reaction of anionic $\operatorname{Ir}^{I}$ NNN complex with $O_{2}$.

Tejel, de Bruin and co-workers observed the backbone oxygenation of a vinylenediamido ligand instead of cyclooctadiene upon reaction of the corresponding anionic $\operatorname{Ir}^{\mathrm{I}}$ complex with dioxygen (scheme II.1.7). ${ }^{[212]}$ Importantly, this reactivity was attributed to the redox non-innocent behavior of the NNN ligand. Hence, the relationship of this iridate(I) with $\mathbf{5}$ initiated this examination on the reactivity of $\mathbf{5}$ with molecular oxygen.

A solution of $\mathbf{5}$ in THF immediately turns red upon stirring under dioxygen (scheme II.1.8). From this solution, the oxygen adduct $\left[\operatorname{Ir}\left(\mathrm{O}_{2}\right)\left(\mathrm{N}\left\{\mathrm{CHCHP}^{t} \mathrm{Bu}_{2}\right\}_{2}\right)\right]$ (10) was 
isolated as a stable compound in around $80 \%$ yield. The NMR spectroscopic characterization reveals the formation of a diamagnetic compound with $C_{2 V}$ symmetry on the NMR timescale at room temperature. In the IR spectrum, a strong band at $910 \mathrm{~cm}^{-1}$ was assigned to the $\mathrm{O}-\mathrm{O}$ stretching vibration by comparison with parent $\mathbf{5}$. This value is at the upper end of the range reported for peroxo ligands and close to the one reported for $\left[\mathrm{Ir}\left(\mathrm{O}_{2}\left(\mathrm{C}_{6} \mathrm{H}_{3}\left\{\mathrm{CH}_{2} \mathrm{P}^{t} \mathrm{Bu}_{2}\right\}_{2}\right)\right]\left(895 \mathrm{~cm}^{-1}\right)\right.$ suggesting a formal iridium(III) oxidation state for 10. ${ }^{[213]}$ The apparently slightly weaker reducing activation of the dioxygen ligand by the $\operatorname{Ir}\left(\mathrm{N}\left\{\mathrm{CHCHP}^{t} \mathrm{Bu}_{2}\right\}_{2}\right)$ compared with $\operatorname{Ir}\left(\mathrm{C}_{6} \mathrm{H}_{3}\left\{\mathrm{CH}_{2} \mathrm{P}^{t} \mathrm{Bu}_{2}\right\}_{2}\right)$ fragment is in line with the $\mathrm{CO}$ stretching vibrations of $6\left(1937 \mathrm{~cm}^{-1}\right)$ vs. $\left[\operatorname{Ir}(\mathrm{CO})\left(\mathrm{C}_{6} \mathrm{H}_{3}\left\{\mathrm{CH}_{2} \mathrm{P}^{t} \mathrm{Bu}_{2}\right\}_{2}\right)\right]$ $\left(1913 \mathrm{~cm}^{-1}\right) \cdot{ }^{[214]}$

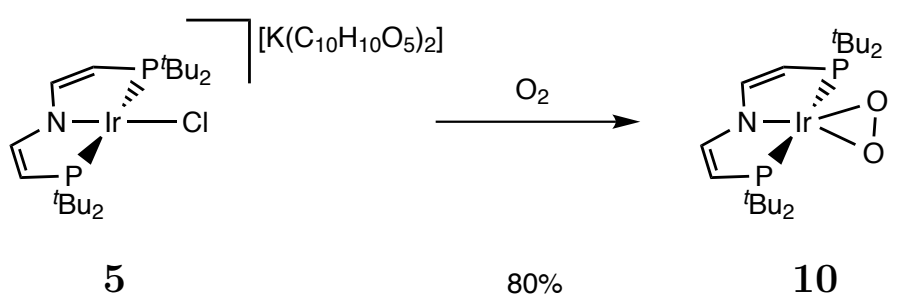

Scheme II.1.8: Activation of dioxygen by anionic iridium(I) chloro complex 5.

The spectroscopic interpretations are corroborated by the molecular structure of $\mathbf{1 0}$ (figure II.1.8). ${ }^{9}$ The crystal exhibited disorder with two superimposed positions of the molecule. While for this reason, the experimental bond lengths and angles should be interpreted with care, some important conclusions regarding $\mathrm{O}_{2}$ bonding from structural parameters can be drawn. Complex 10 exhibits a distorted square-planar geometry and the side-on $\eta^{2}-\mathrm{O}_{2}$ ligand occupies the trans-position to nitrogen with perpendicular orientation to this plane. The weak trans-influence of the dioxygen ligand, similar to chloride in 5, is indicated by the $\mathrm{Ir}-\mathrm{N}$ distance $(2.015(5) \AA)$ and the resulting $\mathrm{P}-\mathrm{Ir}-\mathrm{P}$ bite angle $\left(162.79(4)^{\circ}\right)$. The $\mathrm{O}-\mathrm{O}$ distance $(1.415(7) \AA)$ suggests considerable activation of the dioxygen ligand $\left(d_{O O}=1.21 \AA\right)$ and is close to the typical range found for $\operatorname{Ir}\left(\eta^{2}-\mathrm{O}_{2}\right)$ complexes $(1.43-1.53 \AA) .{ }^{[215]}$ Furthermore, this bond is slightly longer than the ones reported for $\left[\operatorname{Ir}\left(\mathrm{O}_{2}\right)\left\{\mathrm{C}_{6} \mathrm{H}_{3}-2,6-\left(\mathrm{PPR}_{2}{ }_{2}\right)_{2}\right\}\right](1.372(15) \AA)$ and related rhodium dioxygen pincer complexes $\left[\mathrm{Rh}\left(\mathrm{O}_{2}\right)\left(\mathrm{N}\left\{\mathrm{SiMe}_{2} \mathrm{CH}_{2} \mathrm{P}^{t} \mathrm{Bu}_{2}\right\}_{2}\right)\right](1.363(10) \AA)$ and $\left[\mathrm{Rh}\left(\mathrm{O}_{2}\right)\left(\mathrm{C}_{6} \mathrm{Me}_{2} \mathrm{H}\left\{\mathrm{CH}_{2} \mathrm{P}^{t} \mathrm{Bu}_{2}\right\}_{2}\right)\right](1.365(18) \AA) .{ }^{[211,216,217]}$ Hence, the structural fea-

\footnotetext{
${ }^{9}$ Determination of the molecular structure of $\mathbf{1 0}$ was performed by Dr. Christian Würtele.
} 


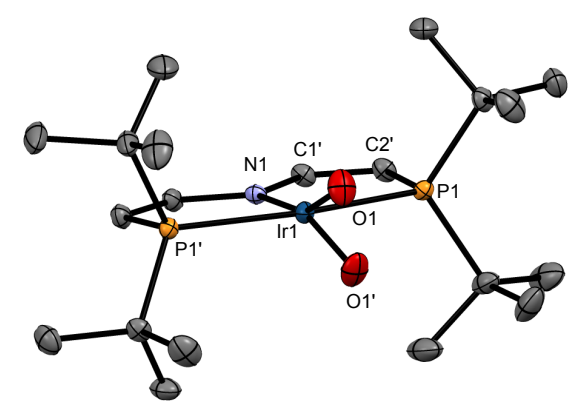

Figure II.1.8.: Molecular structure of 10 in the crystal (one of two orientations within the disordered crystal). Selected bond lengths $[A]$ and angles $\left[^{\circ}\right]$ : Ir1-O1 1.945(3), Ir1-N1 2.015(5), Ir1-P1 2.3461(13), O1-O1'1.415(7), N1-C1 1.407(4), C1-C2 1.352(5); N1-Ir1-P1 158.66(11), O1-Ir1-O1' 42.7(2), P1-Ir1-P1' 162.79(4)

tures are in agreement with assignment to an $\mathrm{Ir}^{\mathrm{III}}$ peroxo complex, as also indicated by IR spectroscopy. However, as Caulton and co-workers carefully stated about the $\mathrm{O}-\mathrm{O}$ bond lengths in such $\eta^{2}-\mathrm{O}_{2}$ complexes, perhaps this parameter is not truly reliable for establishing the charge state, but rather only the degree of back bonding. ${ }^{[216]}$ 


\section{Square-planar iridium nitrido complexes}

As mentioned before (chapter 3.2), the fully dehydrogenated PNP pincer ligand coordinated to iridium provides a suitable platform for the stabilization of multiply bonded ligands. In particular, the vacant $d_{x z}$-based molecular orbital possessing $\pi$-symmetry with respect to the $\mathrm{Ir}-\mathrm{Cl}$ bond in the case of $\mathbf{4}$ encourages investigations on attachment of a nitrido ligand on Ir. Furthermore, $\mathbf{3}$ is expected to be an ideal starting material for the synthesis of stable nitrido precursor complexes such as azides or dbabh complexes, due to its thermal robustness.

The results of this chapter were published in 2012 under the title: „Closed-shell and open-shell square-planar iridium nitride complexes". [200]

\subsection{Iridium(II) PNP azide}

Salt metathesis of $\mathbf{3}$ with sodium azide allowed for the synthesis of iridium(II) azido complex $\mathbf{1 1}$ in high yield (Scheme II.2.1). When $\mathbf{3}$ is stirred in benzene in the presence

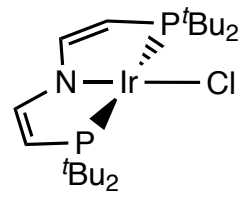

3

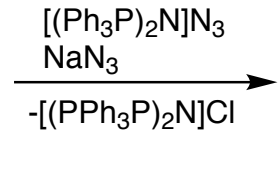

$83 \%$

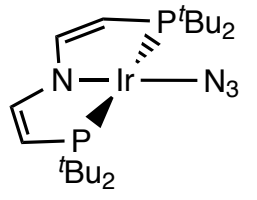

11

Scheme II.2.1: Salt metathesis of 3 with bis(triphenylphosphoranylidene)ammonium azide and excess of sodium azide to 11.

of sodium azide, no reaction is observed even at elevated temperatures over the course of $16 \mathrm{~h}$. The lack of reactivity is attributed to the poor solubility of the azide source in 


\section{Square-planar iridium nitrido complexes}

non-polar solvent. Conversion of $\mathbf{3}$ with the bis(triphenylphosphoranylidene)ammonium azide $\left(\left[\left(\mathrm{Ph}_{3} \mathrm{P}\right)_{2} \mathrm{~N}\right] \mathrm{N}_{3}\right)$ in THF results in color change of the blue solution to green and the formation of one set of three paramagnetically broadened and shifted signals can be observed by ${ }^{1} \mathrm{H}$ NMR spectroscopy (wide infra). Besides, the formation diamagnetic side products is indicated by sharp signals in the ${ }^{31} \mathrm{P}$ NMR spectrum.

The low conversion of the starting material points towards an equilibrium of $\mathbf{3}$ and 11 in solution in the presence of $\left[\left(\mathrm{Ph}_{3} \mathrm{P}\right)_{2} \mathrm{~N}\right] \mathrm{X}\left(\mathrm{X}=\mathrm{N}_{3}, \mathrm{Cl}\right)$. Increasing the excess of $\left[\left(\mathrm{Ph}_{3} \mathrm{P}\right)_{2} \mathrm{~N}\right] \mathrm{N}_{3}$ to 5 equivalents and changing the solvent to acetone shifts the ratio of 3:11 to 1.3:1 ( ${ }^{1} \mathrm{H}$ NMR integration) and full conversion is achieved when 10 equivalents of the azide salt are added.

The expensive $\left[\left(\mathrm{Ph}_{3} \mathrm{P}\right)_{2} \mathrm{~N}\right]^{-}$provides high azide solubility and can be applied as phase transfer catalyst, as demonstrated by full conversion of 3 by conversion with $\left[\left(\mathrm{Ph}_{3} \mathrm{P}\right)_{2} \mathrm{~N}\right] \mathrm{N}_{3}$ : $\mathrm{NaN}_{3}$ in a 1:9 ratio. Full conversion of starting material is achieved after $0.5 \mathrm{~h}$ reaction time and 11 can be obtained after removal of all ionic impurities by stepwise extraction with benzene and pentanes. After lyophilization, $\mathbf{1 1}$ can be obtained in $83 \%$ yield as green powder in analytically pure form.

An ${ }^{15} \mathrm{~N}$ enriched sample of $\mathbf{1 1}\left({ }^{\mathbf{1 4} / \mathbf{1 5}} \mathrm{N}-\mathbf{1 1}\right)$ is obtained when $\mathbf{1 1}$ is stirred with 2 equivalents of $\left[\left(\mathrm{Ph}_{3} \mathrm{P}\right)_{2} \mathrm{~N}\right]^{15} \mathrm{~N}-\mathrm{N}_{2}$ providing ${ }^{\mathbf{1 4} / \mathbf{1 5}} \mathrm{N}-1 \mathbf{1} 33 \%$ enriched with the ${ }^{15} \mathrm{~N}$ isotope in $\alpha$-position to the metal, according to the stoichiometry of the reaction.

$\mathbf{1 1}$ is only moderately stable at room temperature and decomposition is observed under light even in the solid state (vide infra). Hence, $\mathbf{1 1}$ was synthesis at $0{ }^{\circ} \mathrm{C}$ under exclusion of light and stored at $-35{ }^{\circ} \mathrm{C}$ under an argon atmosphere in the dark.

The IR spectrum of 11 exhibits one characteristic band at $2047 \mathrm{~cm}^{-1}$, which is assigned to the azido stretching vibration. No additional bands derived from isotopic labeling could be observed in the IR spectrum of ${ }^{14 / 15} \mathrm{~N}-11$.

11 exhibits three paramagnetically broadened and shifted signals attributable either to the ${ }^{t} \mathrm{Bu}$ groups $(\delta=9.3 \mathrm{ppm})$ or to two sets of $\mathrm{CH}$ backbone protons $(\delta=-5.6$ and -135.9 ppm), respectively, indicating $C_{2 V}$ symmetry on the NMR timescale. The magnetic moment derived by the Evan's method $\left(\mu_{e f f}=2.3 \mu_{B}\right)$ is in agreement with a doublet ground state and similar to the effective magnetic moment of starting material 3. $^{[163,176]}$

The EPR spectrum of $\mathbf{1 1}$ (figure II.2.1) is rhombic with a large anisotropy of the $g$ tensor $\left(g_{11}=3.091, g_{22}=2.066, g_{33}=1.700\right)$ without resolved (super)hyperfine couplings. The experimental $g$-tensor could be reproduced very well by DFT calculations ( $g_{11}=2.878$, $\left.g_{22}=2.080, g_{33}=1.653\right)$ with only slight deviations for $g_{11}$ on the truncated model 


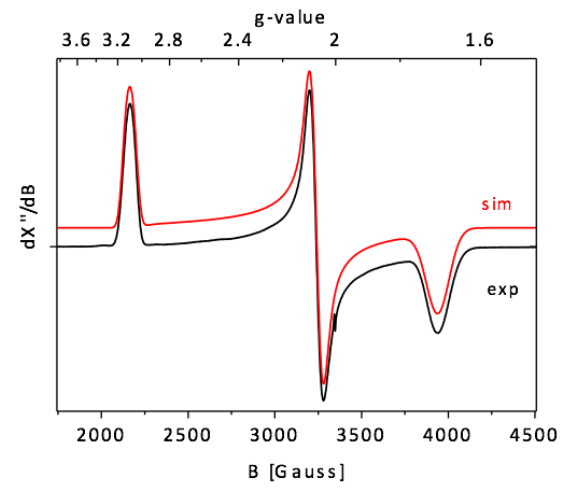

Figure II.2.1.: Experimental (black line) and simulated (red line) EPR spectrum of 11 recorded at $20 \mathrm{~K}$ in a toluene glass (modulation amplitude, $4 \mathrm{G}$; microwave power, $0.2 \mathrm{~mW}$; frequency, $9.377 \mathrm{GHz})$.

complex $\left[\operatorname{Ir}\left(\mathrm{N}_{3}\left\{\mathrm{~N}\left(\mathrm{CHCHPMe}_{2}\right)_{2}\right\}\right] \mathbf{1 1}^{\mathrm{Me}}\right.$ bearing the methyl substituents on the phosphorus atoms. The spin density of $\mathbf{1 1}^{\mathrm{Me}}$ is mainly located at the metal center (ADF: $58 \%$; Turbomole: $71 \%$ ) and is very similar to $3^{[163]}$. Hence, only minor differences in the redox behavior is expected for $\mathbf{3}$ and $\mathbf{1 1} .^{1}$

Single crystals of $\mathbf{1 1}$ could be obtained by slow evaporation of a concentrated pentanes solution to dryness. Attempts to resolve the molecular structure were unsuccessful due to modulation based on disorder of the azido ligand. However, crystals obtained out of a concentrated solution of $\mathbf{1 1}$ in pentanes or hexanes revealed an azido-bridged iridium(II)/iridium(III) dimer. The structure revealed one iridium center in square-planar coordinated by the PNP pincer ligand and one bridging azide ligand, which is further coordinated to a second iridium atom. This second iridium center is in an octahedral coordination geometry with the meridional coordinated PNP pincer ligand, two terminal azido ligands and the bridging azido ligand perpendicular to the Iridium PNP plane. There were no investigation on final resolution of the structure or onto the mechanism responsible for formation of this product. ${ }^{2}$ Due to the disagreement of the molecular structure with the spectroscopic results derived from analytically pure 11, its formation is attributed to decomposition during crystallization and was not further investigated. Putative Iridium(III) azido complex is not expected to be stable. $\mathrm{N}_{2}$ elimination from

\footnotetext{
${ }^{1}$ EPR spectroscopy and DFT calculations were performed in collaboration with Prof. Dr. Bas de Bruin.

${ }^{2}$ Investigation on the molecular structure of $\mathbf{1 1}$ were performed in collaboration with Prof. Dr. Dietmar Stalke.
} 
cationic Iridium(III) azide $\left(\mathrm{S}=0, \Delta \mathrm{G}_{298 K^{0}}=-34.8 \mathrm{kcal} \mathrm{mol}^{-1}\right)$ is calculated to be considerably more exergonic than from neutral $\mathbf{1 1}^{\mathrm{Me}}\left(\mathrm{S}=1 / 2, \Delta \mathrm{G}_{298 K^{0}}=-14.3 \mathrm{kcal} \mathrm{mol}^{-1}\right)$. This can be rationalized easily in terms of stronger $\mathrm{Ir} \equiv \mathrm{N}$ bonding in the resulting nitride for the cationic compound. This trend in increasing $\pi$ bond character with increasing formal oxidation state of the metal was already observed for the iridium PNP chloro system 3 and 4 (wide supra). Hence, no stable cationic iridium azido complex is expected by one electron oxidation of $\mathbf{1 1}$.

\subsection{Iridium(V) PNP nitride}

Chemical oxidation of $\mathbf{1 1}$ results in $\mathrm{N}_{2}$ extrusion and yields in cationic terminal iridium nitrido complex 12 (scheme II.2.2).

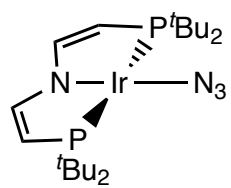

11

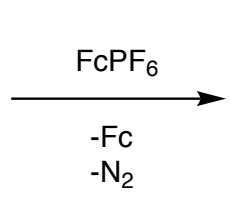

$82 \%$

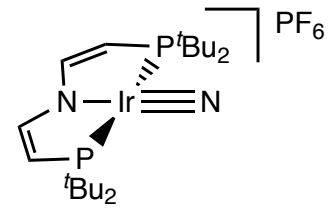

12

Scheme II.2.2: Oxidation of 11 with ferrocenium hexafluorophosphate under $N_{2}$ elimination to cationic iridium( $V)$ nitride complex 12.

Dissolving a mixture of $\mathbf{1 1}$ and $\mathrm{FcPF}_{6}(\mathrm{Fc}=$ ferrocene $)$ in $\mathrm{DCM}$ or acetone results in deep blue solution and gas evolution in time of mixing. After removal of residual starting material and ferrocene (Fc) by extraction with benzene, $\mathbf{1 2}$ can be obtained as dark blue micro crystalline solid in $82 \%$ yield analytically pure after crystallization from DCM/pentanes. Oxidation of ${ }^{\mathbf{1 4} / \mathbf{1 5}} \mathrm{N}-\mathbf{1 1}$ allowed for the isolation of ${ }^{\mathbf{1 4} / \mathbf{1 5}} \mathrm{N}-\mathbf{1 2}$, which is $33 \%$ enriched with the ${ }^{15} \mathrm{~N}$ isotope in the nitrido ligand based on the isotopic distribution in the starting material (vide supra).

Diamagnetism of 12 is indicated by one further signal in the ${ }^{31} \mathrm{P}$ NMR spectrum besides the characteristic heptet of the $\mathrm{PF}_{6}$ anion. The ${ }^{1} \mathrm{H}$ NMR spectrum of $\mathbf{1 2}$ exhibits three characteristic multiplet assignable to the ${ }^{t}$ Bugroups and two sets of pincer backbone protons, respectively. One signal with a chemical shift in the typical range for terminal 

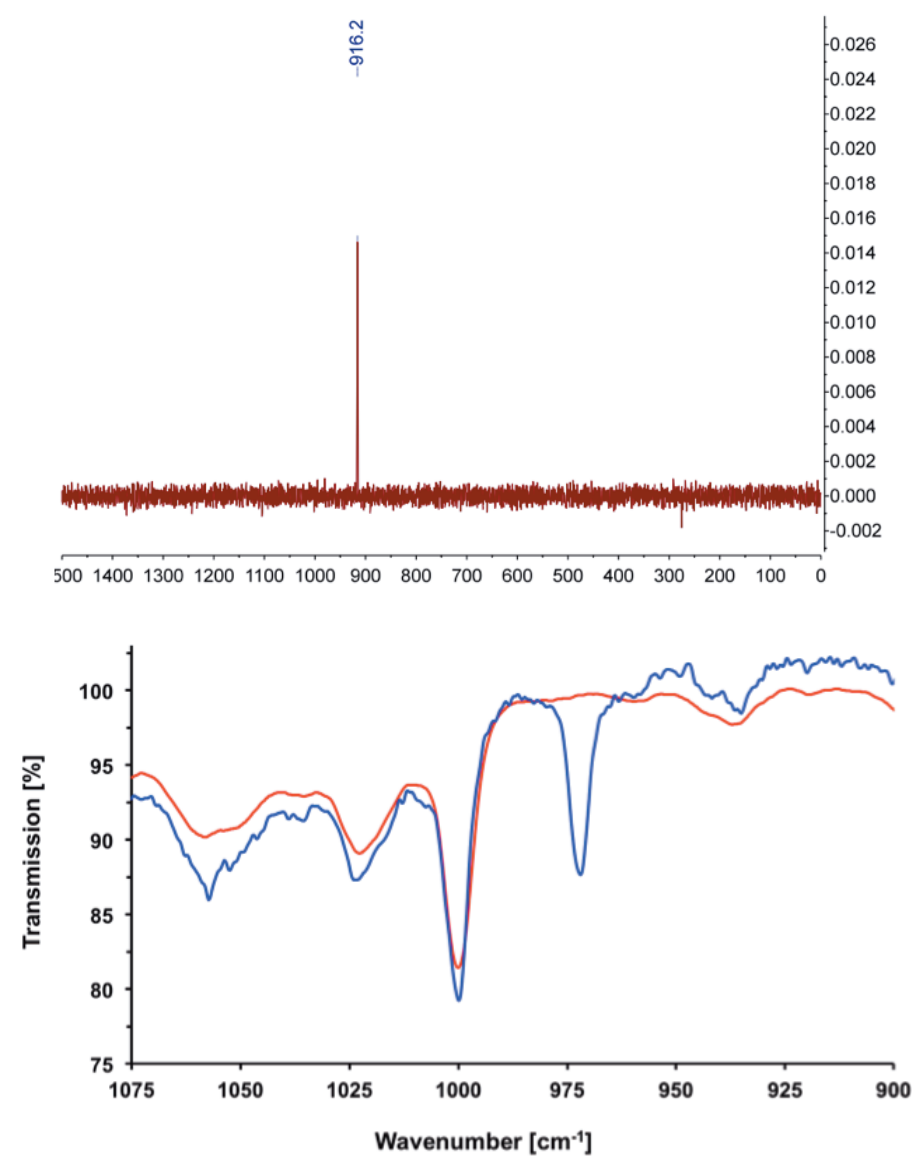

Figure II.2.2.: ${ }^{15} \mathrm{~N} N M R$ spectrum $\left(d_{2}-D C M, R T\right)$ of ${ }^{15 / 14} \mathrm{~N}-12$ (top) and $I R$ spectrum (bottom) of 12 (red line) and of ${ }^{15 / 14} \mathrm{~N}-12$ (blue line).

nitrido complexes in the ${ }^{15} \mathrm{~N}$ NMR spectrum of ${ }^{\mathbf{1 4} / \mathbf{1 5}} \mathrm{N}-\mathbf{1 2}$ (916 ppm vs. ${ }^{15} \mathrm{NH}_{4} \mathrm{NO}_{3}$, figure II.2.2 top) is characteristic for a terminal nitrido moiety.

Comparison of the IR spectra of $\mathbf{1 2}$ and ${ }^{\mathbf{1 4} / \mathbf{1 5}} \mathrm{N}-\mathbf{1 2}$ (figure II.2.2 bottom) allowed for the assignment of a weak band at $999 \mathrm{~cm}^{-1}$ to the $\mathrm{Ir} \equiv{ }^{14} \mathrm{~N}$ stretching vibration, that is shifted to $972 \mathrm{~cm}^{-1}$ in the ${ }^{15} \mathrm{~N}$ enriched sample. The isotopic shift of $\Delta \nu=27 \mathrm{~cm}^{-1}$ compares well with the theoretical value calculated out of the harmonic oscillator approximation $\left(\Delta \nu=26 \mathrm{~cm}^{-1}\right)$. The stretching frequency for $\mathbf{1 2}$ is in good agreement to one reported for iridium nitrido complex $\mathbf{I}\left(958 \mathrm{~cm}^{-1}\right)$. ${ }^{[28]}$

Suitable single crystals of the triflate (= OTf) salt of $\mathbf{1 2}$ could be obtained pentane diffusion in a DCM solution of $\mathbf{1 2}$, that was formed via oxidation of $\mathbf{1 1}$ with AgOTf. 


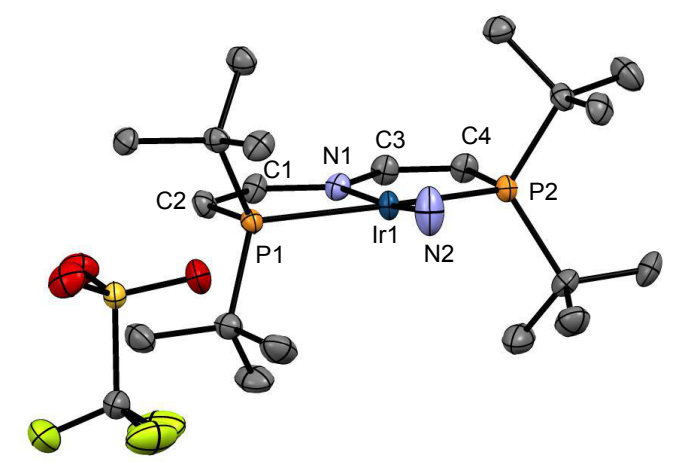

Figure II.2.3.: Molecular structure of 12OTf in the crystal (one out of two crystallographically independent ion pairs; H atoms and co-crystallized DCM omitted for clarity). Representative bond lengths [A] and angles [\% : Ir1-N1 2.041(4), Ir1-N2 1.678(4), Ir1-P1 2.3718(12), Ir1-P2 2.3753(12), N1-C2 1.405(6), N1-C4 1.408(6), C2-C3 1.330(6), C4-C5 1.329(5); N1-Ir1-N2 174.5(2), P1-Ir1-P2 160.13(4).

The molecular structure consistent of two crystallographically independent ion pairs exhibits planar cations with almost linear N-Ir $\equiv \mathrm{N}$ moieties $\left(174.5(2)\right.$ and $\left.173.4(2)^{\circ}\right)$. The short $\mathrm{Ir} \equiv \mathrm{N}$ bonds $(1.678(4)$ and 1.677(4) $\AA$ ) compares well with those of iridium nitride $\mathbf{I}(1.646(9) \AA) .{ }^{[28]}$ The $\mathrm{C}=\mathrm{C}$ double bonds in the pincer-ligand backbone are evidenced by distances that range between $1.320(6)$ and 1.330(6) $\AA$. This rigid ligand framework enforces planar coordination of the dienamido nitrogen atom, in contrast with the stingily pyramidally coordinated amido nitrogen in $\left[\mathrm{Ru}(\mathrm{N})\left\{\mathrm{N}\left(\mathrm{SiMe}_{2} \mathrm{CH}_{2} \mathrm{P}^{t} \mathrm{Bu}_{2}\right\}_{2}\right)\right]^{[29]}$ and $\left[\mathrm{Ru}(\mathrm{N})\left\{\mathrm{N}\left(\mathrm{SiMe}_{2} \mathrm{CH}_{2} \mathrm{P}^{t} \mathrm{Bu}_{2}\right)_{2}\right\}\right]^{[73]}$. Despite the higher formal oxidation state, the Ir- $\mathrm{N}_{\text {amido }}$ bond length in 12 (X-ray, 2.041(4) and 2.032(4) $\AA$ ) is considerably longer than the corresponding distances in iridium(II) and iridium(III) complexes $\mathbf{3}(1.985(2) \AA)$ and $4(1.922(2) \AA)^{[163]}$. This is consequence of the strong nitrido trans-influence. ${ }^{3}$

Electronic structure calculations for $12(\mathrm{~S}=0)$ confirmed the qualitative picture from the isolobal relationship with octahedral $d^{2}$ nitrides (figure II.2.4). As expected for a square-planar complex, the filled orbitals with a predominant $d_{z^{2}}$ and $d_{y z}$ character are energetically low-lying (HOMO-1 and HOMO-3) and the vacant $d_{x^{2}-y^{2}}$ orbital is raised strongly in energy $(\mathrm{LUMO}+3)$. In between, the LUMO and $\mathrm{LUMO}+1$ represent the $\operatorname{Ir} \equiv \mathrm{N} \pi^{*}$ orbitals. The Ir $\equiv \mathrm{N}$ Wiberg bond index (WBI, 2.447) and natural bond order analysis indicate highly covalent $\mathrm{Ir} \equiv \mathrm{N}$ multiple bonding, formed with an $N\left(\mathrm{sp}^{2}\right)$ hybrid ( $\sigma$-bond, $34 \%$ Ir contribution) and two $N(\mathrm{p})$ orbitals ( $\pi$-bonds, $57 \%$ and $58 \%$ Ir con-

\footnotetext{
${ }^{3}$ Determination and refinement of the molecular structure of $\mathbf{1 2}$ in the solid state was performed by Dr. Frank W. Heinemann.
} 
tributions), respectively. Hence, a considerably lower effective oxidation state must be assigned to the metal center, being closer to Iridium(III) than to the formal oxidation number Iridium $(V)$. This electronic structure is reflected by the reactivity which results in electrophilicity of the nitride ligand. ${ }^{4}$

Owing to the $\operatorname{Ir} \equiv \mathrm{N} \pi^{*}$ character of the LUMO, reduction of $\mathbf{1 2}$ was examined to fur-

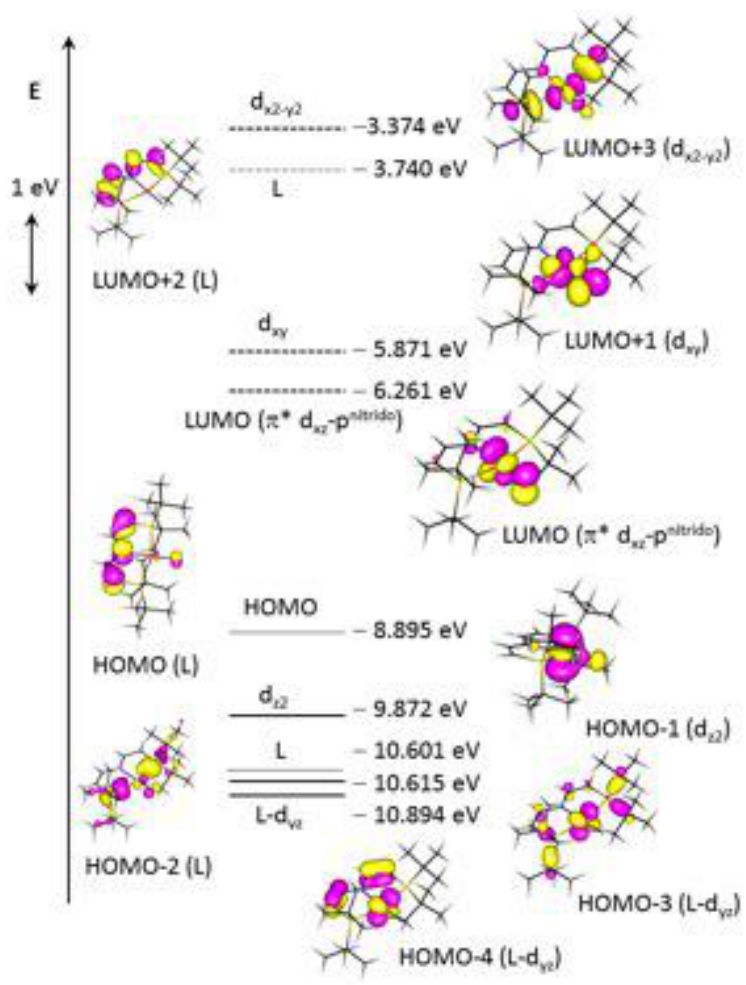

Figure II.2.4.: Frontier Kohn-Sham orbitals of $12(S=0)$, virtual orbitals are indicated with dashed lines and predominantly pincer-ligand centered orbitals are in grey.

ther probe the Ir $\equiv \mathrm{N}$ bonding interaction. The cyclic voltammogram (figure II.2.5) of $\mathbf{1 2}$ in $\mathrm{DCM}$ exhibits a quasi-reversible reduction wave at $-0.9 \mathrm{~V}\left(\mathrm{vs} \mathrm{Fc} / \mathrm{Fc}^{+}\right)$at scan rates $>1 \mathrm{Vs}^{-1}$, which becomes irreversible on slower cycling. Hence, the timescale for decomposition of the immediate reduction product, that is the neutral nitrido complex $\mathbf{1 3}$, is slow enough for spectroscopical characterization (wide infra).

${ }^{4}$ DFT calculations on $\mathbf{1 2}$ were performed by Prof. Dr. Bas de Bruin. 


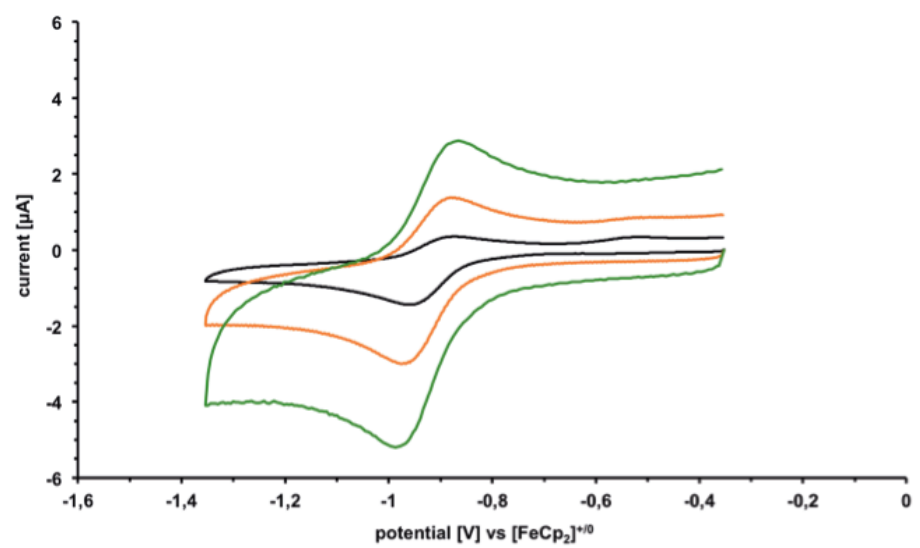

Figure II.2.5.: Cyclic voltammograms of 12 at different scan rates: 1200 (green), 400 (orange), 100 (black) $\mathrm{mVs}^{-1}$ (room temperature, DCM, glassy carbon working electrode,

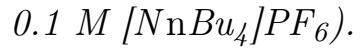

\subsection{Iridium(IV) PNP nitride}

\subsubsection{Spectroscopic characterization}

Based on the electrochemical investigations on $\mathbf{1 2}$ (wide supra), it was suggested that neutral iridium nitrido complex $\mathbf{1 3}$ is stable enough for spectroscopic characterization. Synthetic assess to $\mathbf{1 3}$ was provided either by photolytic $\mathrm{N}_{2}$ extrusion derived from $\mathbf{1 1}$ or by chemical reduction of $\mathbf{1 2}$ (scheme II.2.3).

Chemical reduction of $\mathbf{1 2}$ with $\left[\left(\mathrm{C}_{5} \mathrm{Me}_{5}\right)_{2} \mathrm{Co}\right]$ in $d_{8}$-THF lead to the disappearance of the

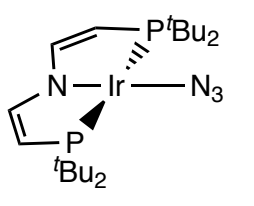

11

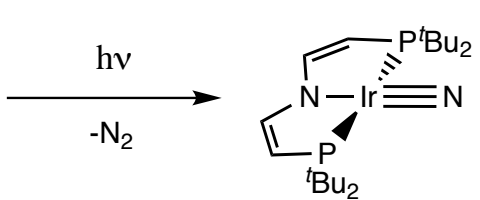

13
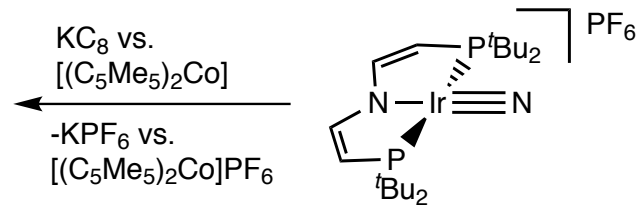

12

Scheme II.2.3: Synthesis of neutral square-planar iridium nitrido complex 13 by photolysis of 11 or reduction of $\mathbf{1 2}$.

${ }^{1} \mathrm{H}$ and ${ }^{31} \mathrm{P}$ NMR signals of starting material at $-40{ }^{\circ} \mathrm{C}$ (figure II.2.6) and the formation of one new set of paramagnetically broadened and shifted signals in $65 \%$ spectroscopic yield. These signals with a chemical shift of $\delta=7.77,-13.4$ and $-63.2 \mathrm{ppm}$ at $-40{ }^{\circ} \mathrm{C}(\delta=$ 
6.55, -9.54 and $-49.2 \mathrm{ppm}$ at room temperature) are attributed to either the ${ }^{t} \mathrm{Bu}$ groups or two set of PNP pincer backbone protons of 13, respectively, according to their intensities. Additionally, diamagnetic signals assigned to decomposition products (wide infra) were already observable even at low temperatures. The same set of signals were obtained by treatment of a sample of $\mathbf{1 1}$ at $-80{ }^{\circ} \mathrm{C}$ with strong UV light for $15 \mathrm{~min}$. The NMR experiments confirms the formation of the same intermediate on either the photolysis or the reduction route and evidences the open-shell character of $\mathbf{1 3}$ and a $C_{2 V}$ symmetric structure on the NMR timescale.

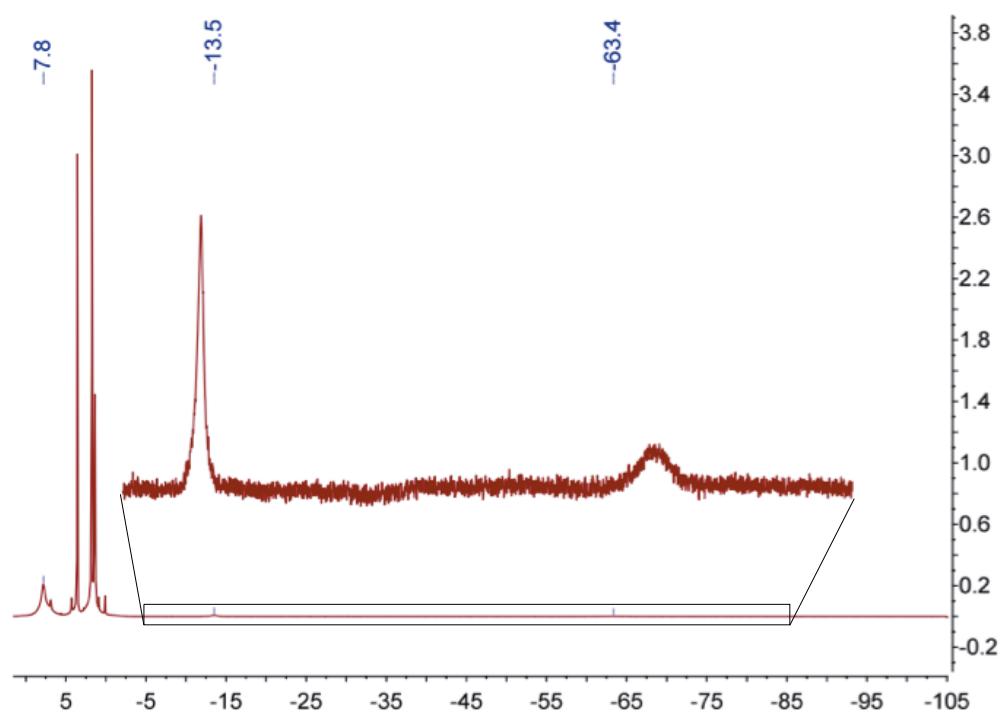

Figure II.2.6.: ${ }^{1} \mathrm{H}$ NMR spectrum of 13 at $-40{ }^{\circ} \mathrm{C}$ in $\mathrm{d}_{8}$-THF obtained by reduction of 12 with $\left[\left(\mathrm{C}_{5} \mathrm{Me}_{5}\right)_{2} \mathrm{Co}\right]$.

Comparison of the IR spectra (figure II.2.7) obtained by irradiation of a $\mathrm{KBr}$ disc containing 11 and ${ }^{\mathbf{1 5} / \mathbf{1 4}} \mathrm{N}-11$ allows for the assignment of the $\operatorname{Ir} \equiv{ }^{14} \mathrm{~N}$ stretching vibration of 13 to a weak band at $901 \mathrm{~cm}^{-1}$. The isotopic shift of the $\mathrm{Ir} \equiv{ }^{15} \mathrm{~N}$ stretch $\left(\nu=874 \mathrm{~cm}^{-1}\right)$ with $\Delta \nu=27 \mathrm{~cm}^{-1}$ compares well with the theoretic value calculated by the harmonic oscillator approximation of $\Delta \nu=28 \mathrm{~cm}^{-1}$. The bathochromic shift of the stretching vibration is indicative for lowering the bond order within the $\mathrm{Ir} \equiv \mathrm{N}$ moiety.

Photolysis of $\mathbf{1 1}$ in toluene glass $(20 \mathrm{~K})$ and motoring by X-band EPR spectroscopy led to the gradual disappearance of its signals and clean formation of new signals assigned to $\mathbf{1 3}$. The spectrum of $\mathbf{1 3}$ (figure II.2.8 right) shows a rhombic signal with large $g$ - 


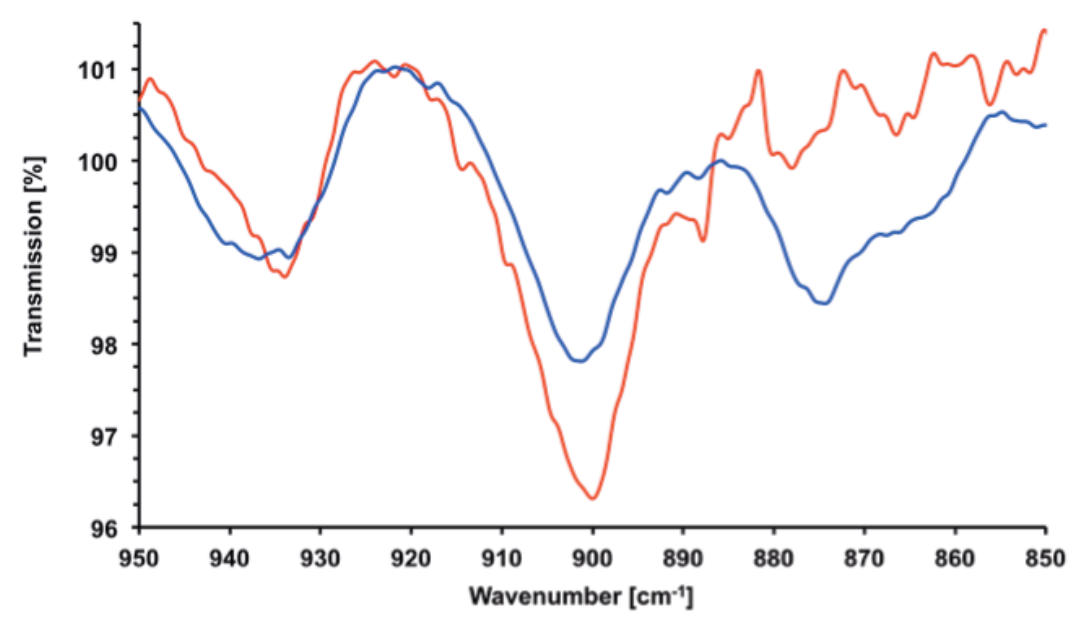

Figure II.2.7.: IR spectra of $\mathrm{KBr}$ disks of 11 (red line) and ${ }^{15 / 14} \mathrm{~N}$-11 (blue line) after irradiation. The bands shown are assigned to the $I r \equiv{ }^{14} \mathrm{~N}$ and $\operatorname{Ir} \equiv{ }^{15} \mathrm{~N}$ stretching vibrations.

anisotropy $\left(g_{11}=1.885, g_{22}=1.631\right.$ and $\left.g_{33}=1.320\right)$ without resolved (super)hyperfine couplings. Remarkably, all $g$-values of this species are well below $g=g_{e}=2.00$, pointing towards strong spin-orbit coupling interactions with MOs based on empty $d$ orbitals, but negligible spin-orbit coupling interactions with MOs based on filled $d$ orbits. ${ }^{5}$

Furthermore, the nitrido ${ }^{14} \mathrm{~N}$ hyperfine interactions (HFIs, $A_{11}^{y}=-26.5, A_{22}^{z}=63.5$ and $A_{33}^{x}=-62.0$ ) and nuclear quadrupole interactions (NQIs, $P_{11}^{y}=2.70, P_{22}^{z}=-0.71, P_{33}^{x}$ $=-2.31)$ were derived by X-band Davies electron-nuclear double resonance (ENDOR) spectroscopy (figure II.2.8). ${ }^{6}$

The positive HFI along $g_{22}(z)$ suggests large excess of $\alpha$-spin along the z-axis, whereas the negative HFI's along $g_{11}(y)$ and $g_{33}(x)$ are attributed to spin polarization and thus creating $\beta$-spin directing along the $x$ and $y$ molecular axes. This large $\alpha$-spin predicts alignment of the orientation of the SOMO with the $z$-axis of the molecule. The polarization mechanism is assigned to exited state admixture of orbital angular momentum via spin orbit coupling (SOC) of the SOMO with solely unoccupied molecular orbitals. The large negative value of the HFI in $\mathrm{A}_{33}^{x}$ is rationalized in terms of spin polarization in $x$-direction. Hence, the molecular orbital in $x$-direction is attributed to an empty MO,

\footnotetext{
${ }^{5}$ EPR spectroscopy was performed by Prof. Dr. Bas de Bruin.

${ }^{6}$ ENDOR spectroscopy was performed by Dr. Edward J. Reijerse.
} 

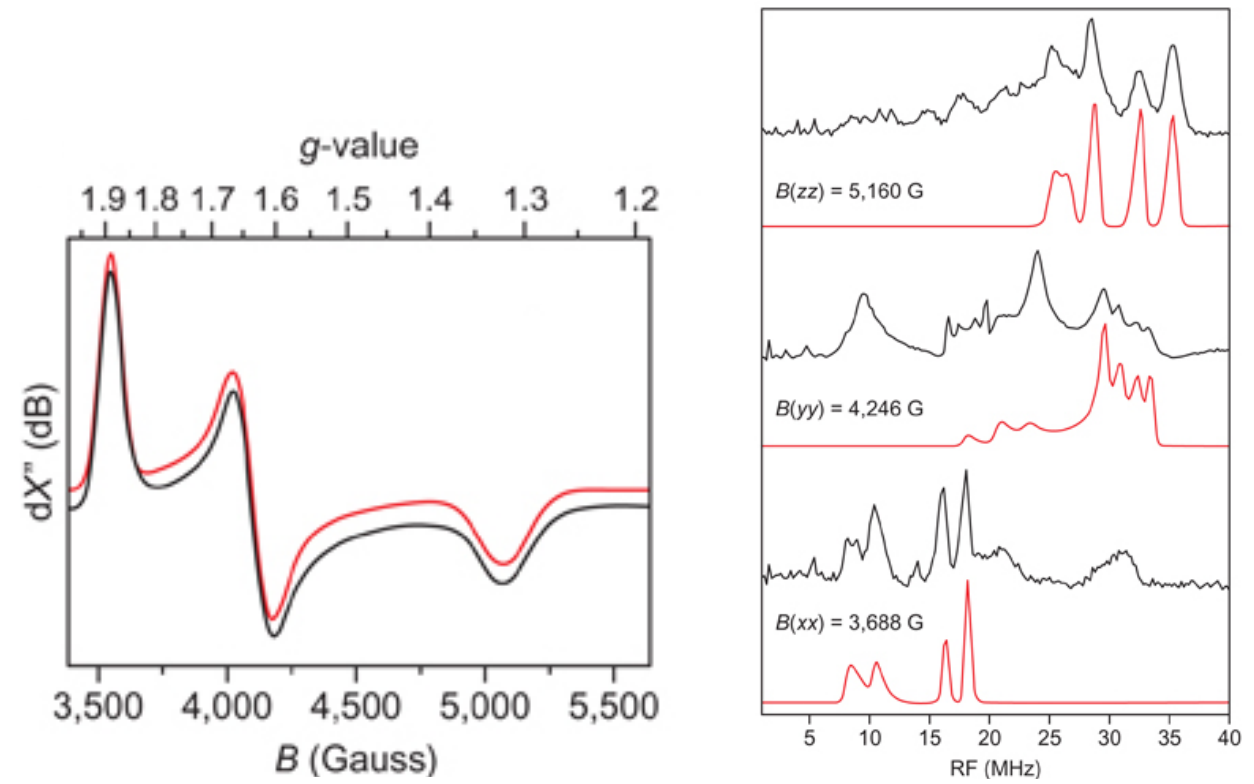

Figure II.2.8.: Experimental (black line) and simulated (red line) EPR spectra (left) of nitrido radical complex 13 after ultraviolet photolysis (120 minutes) of 11 (20 K; toluene glass; modulation amplitude, 4 Gauss; microwave power, $0.2 \mathrm{~mW}$; frequency, 9.377 GHz) and experimental X-band Davies-ENDOR spectra of 12 (black line) (right) with simulation of the ${ }^{14} N$ HFIs (red line) at $g_{11}^{y}, g_{22}^{z}$ and $g_{33}^{x}$ observer positions. Spectra were recorded at $6 \mathrm{~K}$ in a toluene glass.

with respect to the low corresponding $g$ value. The energy difference of a $g$-value from the value of the free electron $g_{e}$ is dependent following equation 2.1 on the SOC constant of the corresponding metal $(\lambda)$, the energy difference of SOMO and mixing molecular orbital $(\Delta)$ and a factor $(n)$, which depends on the symmetry of the mixing orbitals. The sign of $\lambda$ is positive when mixing with filled orbitals and negative when the orbitals are empty. Hence, a shift to lower $g$-values (negative sign) is obtained by mixing with empty MOs and vice versa. ${ }^{[176]}$

$$
g=g_{e} \pm \frac{n \cdot \lambda}{\Delta}
$$

\subsubsection{DFT characterization}

Complex 13 was examined with DFT computations $(S=1 / 2)$ to rationalize the spectroscopic parameters and to analyze the electronic structure. The computed structural features are close to those of iridium(V) nitride 12, but with a slightly longer $\mathrm{Ir} \equiv \mathrm{N}$ bond $(1.727 \AA)$, as a consequence of the injection of an electron into an $\mathrm{Ir} \equiv \mathrm{N}$ anti-bonding 
orbital. Accordingly, the Ir $\equiv \mathrm{N}$ WBI is reduced by $\Delta_{W B I}=0.372$ on reducing 12 to 13. Weakening of the $\mathrm{Ir} \equiv \mathrm{N}$ bond was also confirmed experimentally by vibrational spectroscopy (wide supra). The bathochromic shift derived by reduction of $\mathbf{1 2}$ to $\mathbf{1 3}$ of $\sim 100 \mathrm{~cm}^{-1}$ is in excellent agreement with DFT calculations $\left(\nu=903 \mathrm{~cm}^{-1}, \Delta \nu=\right.$ $\left.96 \mathrm{~cm}^{-1}\right)$.
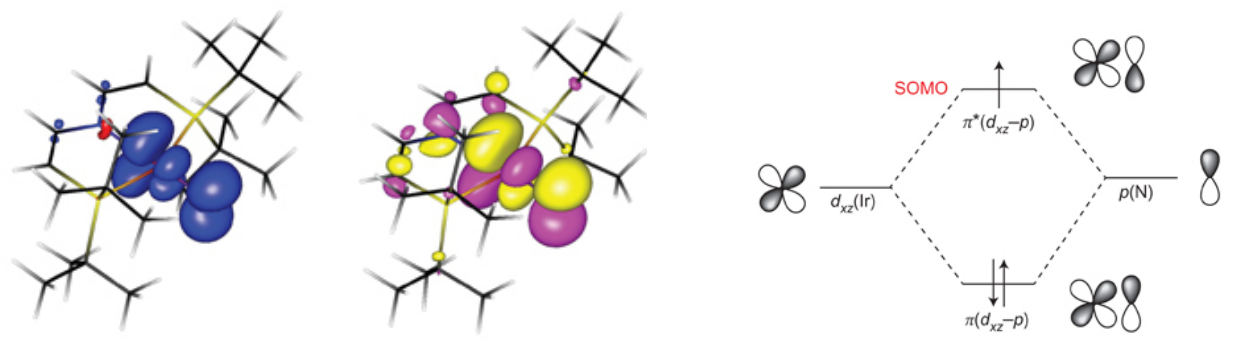

Figure II.2.9.: Spin density plot (left) and SOMO (middle) of 13 and schematic representation of the important orbital interactions that lead to the SOMO.

Accordingly, the SOMO and the Muliken spin density distribution of $\mathbf{1 3}$ strongly resemble the LUMO $\left(\pi_{I r \equiv N}^{*}\right)$ of $\mathbf{1 2}$. Nearly covalent $\operatorname{Ir}\left(d_{x z}\right)$ and $\mathrm{N}\left(p_{z}\right)$ contributions to the SOMO of 13 effect an almost even delocalization of the spin density ( $\sim 40 \%$ localized on Ir and $\sim 50 \%$ on the nitrido ligand), which suggests substantial $\left\{\mathrm{Ir}=\mathrm{N}^{\bullet}\right\}$ 'nitridyl' ligand radical character. Hence, reduction of $\mathbf{1 2}$ is perceived as a partial reduction of both, the metal center and the ligand. Most importantly, the DFT-calculated EPR parameters of 13, such as the $g$-tensor, the ${ }^{14} \mathrm{~N}$ HFI tensor and the NQI tensor, are in excellent agreement with the experimental values (table II.2.1). This gives strong confidence in the accuracy of the applied DFT methods, because EPR parameters are generally very sensitive to small geometric and electronic structure changes.

Analysis of the frontier orbitals of $\mathbf{1 3}$ also provides a basis to rationalize qualitatively the EPR results. As for the LUMO and LUMO +1 of $\mathbf{1 2}$, the SOMO and LUMO of 13 are nearly degenerate and have the appropriate rotation symmetry for optimal spinorbit coupling. In contrast, the large energy separation of the SOMO with MOs based on filled $d$ orbitals, the distortion of the SOMO and the filled $d_{y z}$-based HOMO-3 and HOMO-4 from the considerable ligand character and the opposite spin-orbit interactions of the SOMO with the empty $d_{x^{2}-y^{2}}$ and filled $d_{z^{2}}$ orbitals (orbital rotation method) ${ }^{[176]}$ quench the spin-orbit coupling interactions between the SOMO and the filled 'metal $d$ 
Table II.2.1.: Experimental (spectral simulation, HFCs in $M H z$ ) and DFT (ADF, BP86, ZORA-TZP, geometry optimized with turbomole, b3-lyp, def2-TZVP) calculated EPR parameters of $\mathbf{1 3}$.

\begin{tabular}{cccc}
\hline$g$-values & $g_{11}(y)$ & $g_{22}(z)$ & $g_{33}(x)$ \\
\hline EXP & 1.885 & 1.632 & 1.320 \\
DFT & 1.862 & 1.582 & 1.321 \\
\hline${ }^{14} \mathrm{~N}_{\text {Nitrido }} \mathrm{HFI}$ & $A_{11}^{y}$ & $A_{22}^{z}$ & $A_{33}^{x}$ \\
\hline $\mathrm{EXP}$ & -26.5 & +63.5 & -62.0 \\
$\mathrm{DFT}$ & -19 & +49 & -60 \\
\hline $\mathrm{NQI}$ & $P_{11}^{y}$ & $P_{22}^{z}$ & $P_{33}^{x}$ \\
\hline $\mathrm{EXP}$ & +2.70 & -0.39 & -2.31 \\
$\mathrm{DFT}$ & +3.10 & -0.71 & -2.40 \\
\hline
\end{tabular}

orbitals', and thus provide a reasonable explanation for the fact that all EPR $g$-tensor components of $\mathbf{1 3}$ are considerably smaller then the free electron value. Similarly, the rhombicity of the nitrido ${ }^{14} \mathrm{~N}$ HFI tensor can be attributed to a considerable orbital contribution to the HFI tensor.

The detection and relatively high thermal stability of $\mathbf{1 3}$ are remarkable. Open-shell, terminal nitrido complexes are very rare in general, in particular for the platinum metals. The formation of $\left[\mathrm{Ru}^{\mathrm{V}}(\mathrm{N})(\mathrm{imp})\right]^{2-}$ (imp = mess-octamethylporphyrinogen) by reduction of the nitrido complex $\left[\mathrm{Ru}^{\mathrm{IV}}(\mathrm{imp})\right]^{-}$has been reported, but the electronic structure of $\left[\mathrm{Ru}^{\mathrm{V}}(\mathrm{N})(\mathrm{imp})\right]^{2-}$ was not examined. ${ }^{[218]}$ The associated elongation of the $\mathrm{Ru} \equiv \mathrm{N}$ bond length by about $0.2 \AA$ possibly indicates a weakening of this bond on population of a $\mathrm{Ru} \equiv \mathrm{N} \pi^{*}$ orbital. For the $\mathrm{Ru}_{2}$ paddlewheel nitrido complex $\left[\mathrm{Ru}_{2}(\mathrm{~N})(\mathrm{PhNCHNPh})_{4}\right]$, EPR data and DFT computations indicate a predominant metal radical character. ${ }^{[219]}$ Notably, in contrast to $\mathbf{1 3}$ or $\left[\mathrm{Ru}^{\mathrm{V}}(\mathrm{N}) \mathrm{L}_{5}\right]\left(\mathrm{L}=\mathrm{NH}_{3}\right.$, pyridines, $\left.\mathrm{CO}, \mathrm{Cl}^{-}\right)$, the postulated yet unobserved intermediates in $\mathrm{N}-\mathrm{N}$ coupling, $\left[\mathrm{Ru}_{2}(\mathrm{~N})(\mathrm{PhNCHNPh})_{4}\right]$, decomposes by insertion into aromatic $\mathrm{C}-\mathrm{H}$ bonds. ${ }^{[220]}$ Therefore, it is obscurs as to whether this principle reactivity can be attributed to the features of the (ground state) electronic structures.

\subsection{Iridium(I) PNP dinitrogen complexes}

When a sample of $\mathbf{1 3}$ is kept at room temperature, the formation of two new diamagnetic products 14 and 7 can be observed by ${ }^{1} \mathrm{H}$ and ${ }^{31} \mathrm{P}$ NMR spectroscopy. 7 can be 
synthesized independently by heating a solution of $\mathbf{1 1}$, or by stirring iridium(I) chloro complex $\mathbf{5}$ in solution under $\mathrm{N}_{2}$ atmosphere (chapter 1.3).

The ${ }^{1} \mathrm{H}$ and ${ }^{13} \mathrm{C}$ NMR spectroscopic features of $\mathbf{1 4}$ and $\mathbf{7}$ are nearly identical and indicate $\mathrm{C}_{2 V}$ symmetry on the NMR timescale. However, both compounds can be distinguished easily by their ${ }^{31} \mathrm{P}$ NMR chemical shifts. Furthermore, the ${ }^{15} \mathrm{~N}$ NMR spectrum of a mixture of both 14 and 7 prepared via reduction of ${ }^{14 / 15} \mathrm{~N}-12$ (figure II.2.10) reveals three signals assignable to either the two isotopologues of terminal $\mathrm{N}_{2}$ complex $7{ }^{15} \mathrm{~N}$ labelled either in $\alpha$ or $\beta$ position two the metal or to ${ }^{15} \mathrm{~N}$ labelled $\mu-\mathrm{N}_{2}$ bridged Ir dimer.
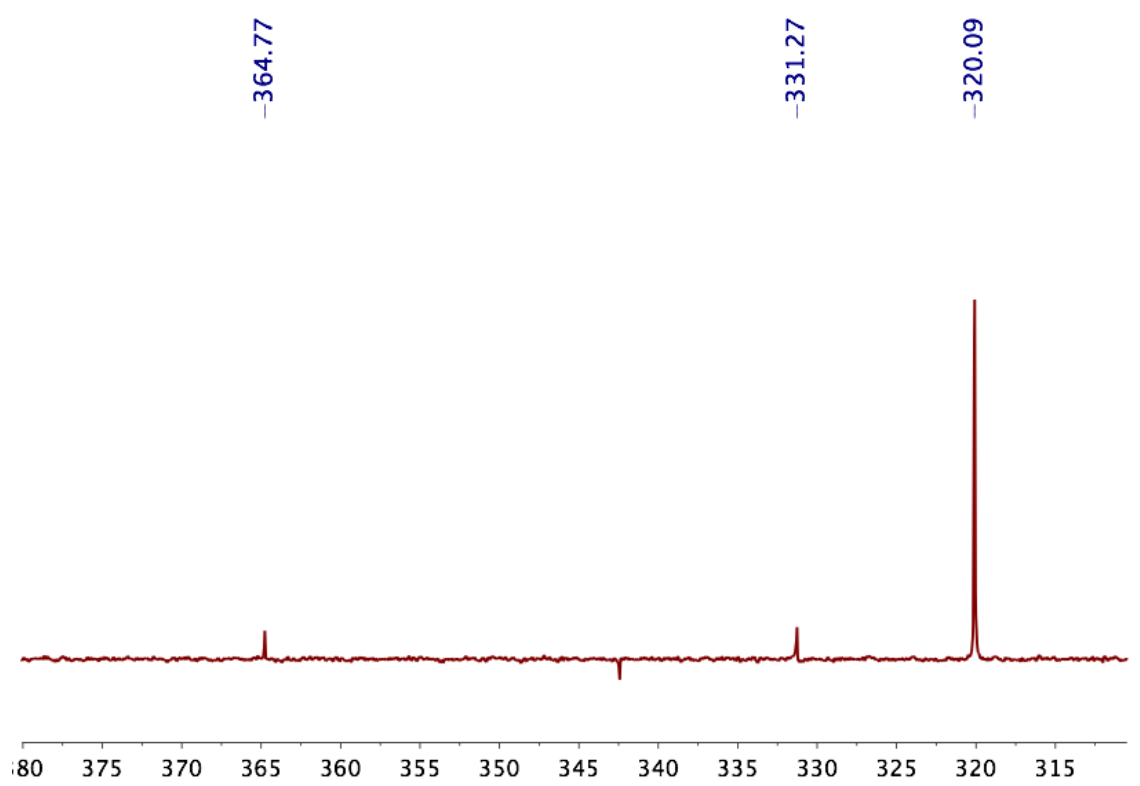

Figure II.2.10.: ${ }^{15} \mathrm{~N} N M R$ spectrum $\left(d_{8}-T H F,-20{ }^{\circ} \mathrm{C}\right)$ obtained after the reaction of ${ }^{15} / 14 \mathrm{~N}-12$ with $K C_{8}$ under an atmosphere of Argon.

There is one intense stretching vibration at $2077 \mathrm{~cm}^{-1}\left(\nu\left({ }^{14} \mathrm{~N} \equiv{ }^{14} \mathrm{~N}\right)\right)$ observable in the IR spectrum of 7 . Irradiation of a $\mathrm{KBr}$ disk containing ${ }^{15 / 14} \mathrm{~N}-11$ leads to the appearance of two additional bands 2040 and $2003 \mathrm{~cm}^{-1}$ (figure II.2.11), which could be further assigned to ${ }^{15} \mathrm{~N} \equiv{ }^{14} \mathrm{~N}$ and ${ }^{15} \mathrm{~N} \equiv{ }^{15} \mathrm{~N}$ stretching vibration, respectively. The isotopic shifts of $\Delta \nu\left({ }^{14} \mathrm{~N} \equiv{ }^{15} \mathrm{~N}\right)=34 \mathrm{~cm}^{-1}$ and $\Delta \nu\left({ }^{15} \mathrm{~N} \equiv{ }^{15} \mathrm{~N}\right)=71 \mathrm{~cm}^{-1}$ are in excellent agreement with the values calculated by the harmonic oscillator approximation $\left(\Delta \nu\left({ }^{14} \mathrm{~N} \equiv{ }^{15} \mathrm{~N}\right)=\right.$ $\left.35 \mathrm{~cm}^{-1} ; \Delta \nu{ }^{15} \mathrm{~N} \equiv{ }^{15} \mathrm{~N}=70 \mathrm{~cm}^{-1}\right)$.

Single crystals suitable for the determination of the molecular structure of $\mathbf{7}$ and $\mathbf{1 4}$ by X- 


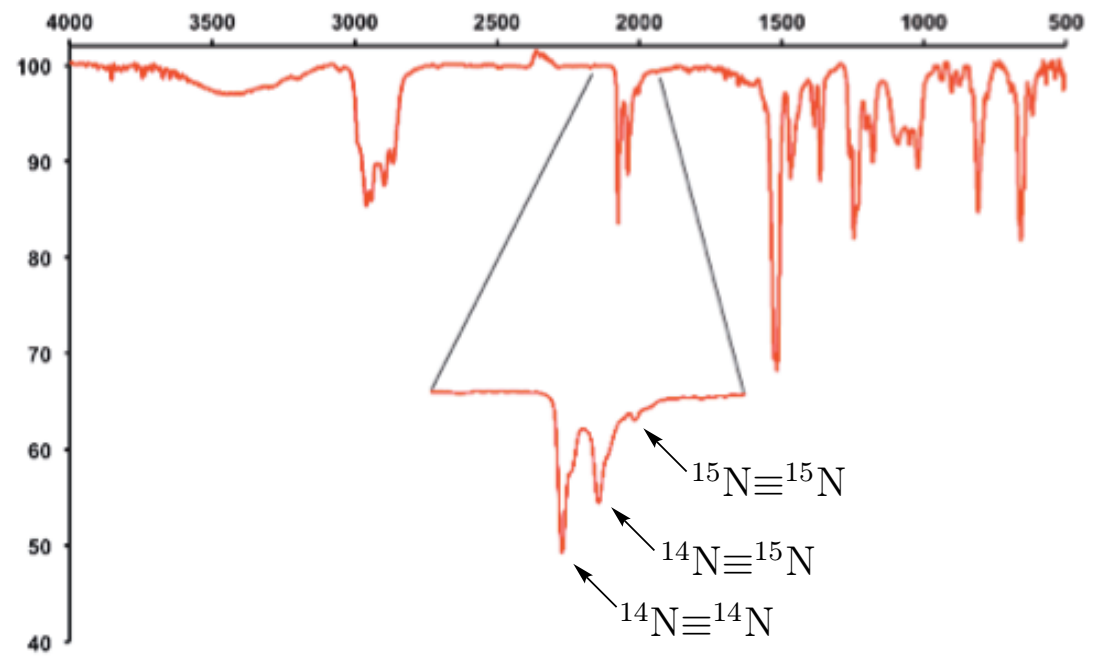

Figure II.2.11.: IR spectrum of a $K B r$ disk of ${ }^{15 / 14} \mathrm{~N}$-11 after irradiation.

ray diffraction experiments were obtained by evaporation of a concentrated solution of either $\mathbf{7}$ or $\mathbf{1 4}$ in pentane to dryness. ${ }^{7}$ The molecular structures of $\mathbf{7}$ and $\mathbf{1 4}$ (figure II.2.12) are in agreement with the spectroscopic characterization and exhibit similar structural features to those reported for related $\mathrm{Ir}^{\mathrm{I}} \mathrm{PCP} \mathrm{N}_{2}\left(\mathrm{PCP}=2,6-\left({ }^{t} \mathrm{Bu}_{2} \mathrm{PCH}_{2}\right)_{2} \mathrm{C}_{6} \mathrm{H}_{3}\right)$ complexes. ${ }^{[201]}$

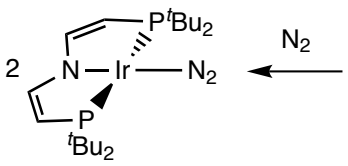

7

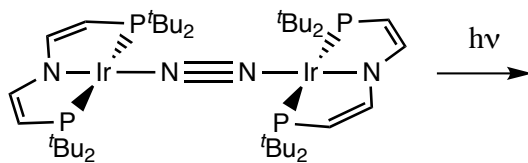

14

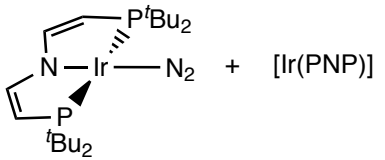

7

Scheme II.2.4: Decomposition pathways of 14.

The dependence of the $\mathbf{1 4} / \mathbf{7}$ ratio on the synthetic pathway is attributed to the amount of free $\mathrm{N}_{2}$ present in the system (scheme II.2.4). One equivalent of dinitrogen is released upon photolytic cleavage of the azido ligand of $\mathbf{1 1}$. On the reduction pathway, only minor traces of dinitrogen in the inert gas can induce the cleavage of the dimer. The observed 

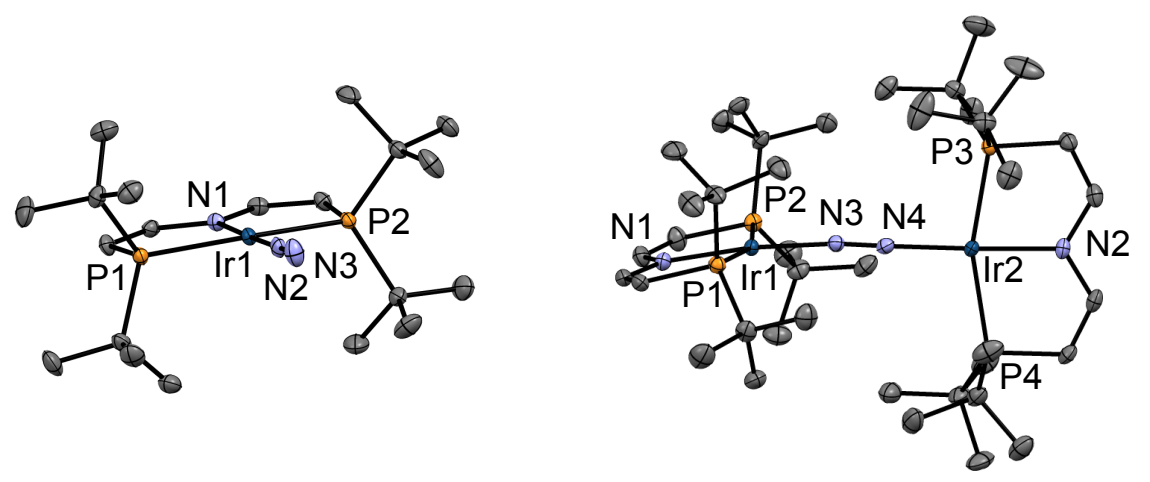

Figure II.2.12.: Molecular structure of 7 (left) and 14 (right) in the crystal from single crystal diffraction. Representative bond lengths $[\AA]$ and angles $~_{\circ} \mathrm{T}$ : 7 Ir-N1 2.019(3), Ir-N2 1.885(3), Ir-P1 2.3062(8), Ir-P2 2.3114(8), N1-N2 1.109(4); N1-Ir-N2 178.1(2); P1-Ir-P2 164.15(3), Ir-N2-N3 178.6(3); 14 Ir1-N1 2.041(3), Ir1-N3 1.937(3), Ir1-P1 2.3134(8), Ir1-P2 2.3354(9), N3-N4 1.135(4), Ir2-N2 2.035(4), Ir2-N4 1.933(3), Ir2-P3 2.3364(8), Ir2-P4 2.3443(8); N1-Ir1-N3 173.6(2)m P1-Ir1-P2 161.11(3), N2-Ir2-N4 171.0(1), P3-Ir2-P4 160.09(3), Ir1-N3-N4 174.6(2), Ir2-N4-N3 172.3(3).

quantities of $\mathbf{7}$ origin from heterolytic light induced cleavage of $\mathbf{1 4}$ into $\mathbf{7}$ and a three coordinate $\left[\operatorname{Ir}^{\mathrm{I}}(\mathrm{PNP})\right]$.

Attributed to this sensitivity, the isolation of $\mathbf{1 4}$ in analytically pure form was not successful. However, the identity of $\mathbf{1 4}$ was confirmed by high resolution mass spectrometry. Analysis of a freshly prepared solution of $\mathbf{1 4}$ obtained by the reduction route, the main product found in the mass spectrum with $m / z=1125.4681\left(\left[\mathrm{M}+\mathrm{H}^{+}\right]\right.$calcd. for $\mathrm{C}_{40} \mathrm{H}_{81} \mathrm{~N}_{4} \mathrm{P}_{4} \mathrm{Ir}_{2}$ 1125.4643) could be assigned to the molar peak of 14 with one additional proton added.

\subsection{Investigations on the nitride coupling reaction}

The decay of $\mathbf{1 3}$ was monitored by ${ }^{1} \mathrm{H}$ NMR spectroscopy (figure II.2.13). A solution of 11 in $d_{8}$-THF containing naphthalene as internal standard was irradiated for 15 min at $-80{ }^{\circ} \mathrm{C}$ and the decay of the ${ }^{1} \mathrm{H}$ NMR signals of the tert-butyl groups of the resulting $\mathbf{1 3}$ was monitored over the course of $1 \mathrm{~h}$.

\footnotetext{
${ }^{7}$ X-Ray diffraction experiments were performed by Dr. Frank W. Heinemann.
} 


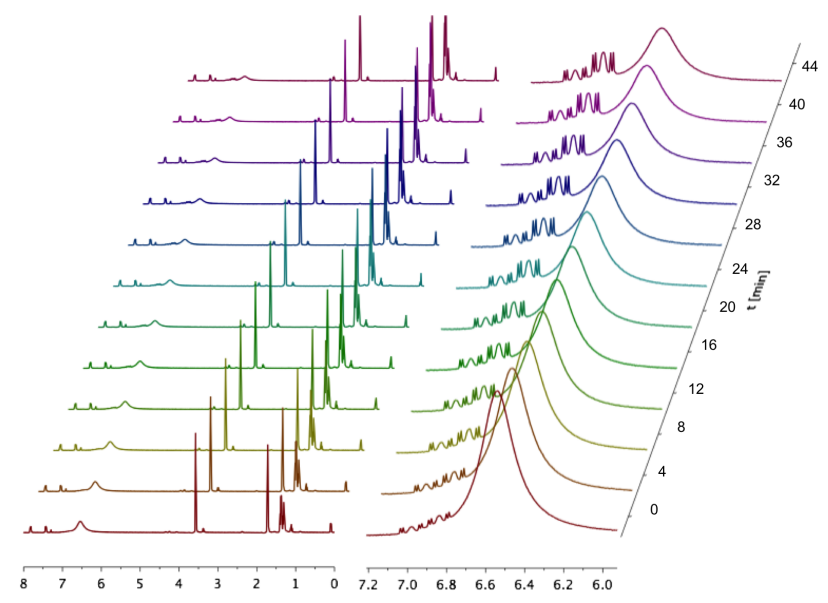

Figure II.2.13.: ${ }^{1} H$ NMR spectra following the decay of 13 in $\mathrm{d}_{8}$-THF at room temperature after photolysis of 11 at $-60{ }^{\circ} \mathrm{C}$ (right: expansion; left: full spectrum).

A linear dependence of the reciprocal concentration of $\mathbf{1 3}$ vs. time indicates decay of $\mathbf{1 3}$ following second order rate law. A reaction rate of $k_{298 K}=1.1(2) \mathrm{M}^{-1} \mathrm{~min}^{-1}$ was found and the half life time of $\mathbf{1 3}$ was determined to 20 minutes at room temperature. Therefore, the spectroscopic and kinetic results suggest that 13 dimerizes by bimolecular, oxidative nitride coupling to the dimeric, labile complex 14, which is partially trapped as monomer $\mathbf{7}$ in the presence of additional $\mathrm{N}_{2}$, for example from photolytic azide cleavage.

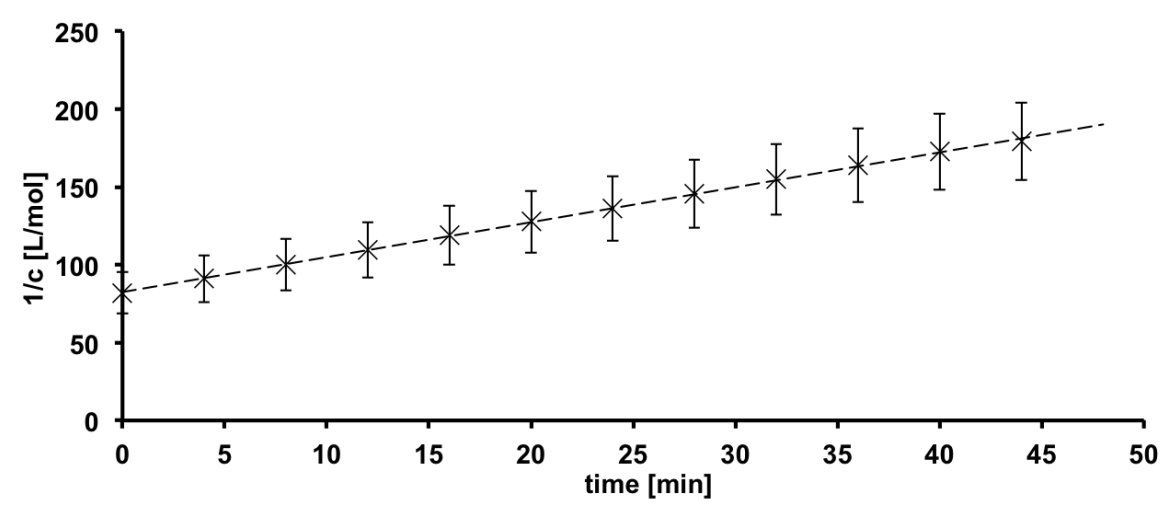

Figure II.2.14.: Kinetic plot (reciprocal concentration c versus time) of the decay of 13 and least squads fit used for estimation of the rate constant (dashed line). 
This interpretation was corroborated by DFT computations. Nitride coupling of the model complex $13^{\mathrm{Me}}$ to $14^{\mathrm{Me}}$ was calculated to be energetically favorable by $\Delta G_{298 K}^{0}=$ $-108 \mathrm{kcal} \mathrm{mol}^{-1}$ and an open-shell singlet transition state $\left(\mathbf{T S}_{\mathrm{BS}}\right)$ was located at $\Delta G_{298 K}^{\ddagger}$ $=+14 \mathrm{kcal} \mathrm{mol}^{-1}$. As expected for such a strongly exergonic reaction, the computed geometric parameters and bond orders of the transition state are close to those of the starting complex $\mathbf{1 3}^{\mathrm{Me}}$. The two $\left[\mathrm{Ir}(\mathrm{N})\left\{\mathrm{N}\left(\mathrm{CHCHPMe}_{2}\right)_{2}\right\}\right]$ fragments approach each other with an almost co-planar 'zig-zag' [(PNP)IrN ...NIr (PNP)] arrangement, which is very similar to the transition state proposed for $\mathrm{N}_{2}$ cleavage with molybdenum anilido complexes ${ }^{[108]}$. This encounter permits the out-of-plane interactions of the two SOMOs, and thus leads to $\mathrm{N}-\mathrm{N}$ bond formation via radical-radical coupling. It has been pointed out previously that in-plane orbital interactions involving $N$ lone pairs and vacant $\mathrm{Ir}-\mathrm{N}$ $\pi^{*}$ orbitals stabilize such a zig-zag transition state for nitride coupling. ${ }^{[221]}$ However, in the present case the analysis of the $\mathbf{T} \mathbf{S}_{\mathbf{B S}}$ orbitals did not reveal any significant in-plane interactions. Instead, they became apparent in constrained-geometry optimizations at a later stage on the reaction coordinate with shorter $\mathrm{N}-\mathrm{N}$ distances prior to completion of the overall $6 \mathrm{e}^{-}$redox process. Hence, this mechanism is, at least in part, determined by the radical character of $\mathbf{1 3}$. A polar 'semi-bent' transition state with nucleophilic attack of one nitrido ligand at the $\mathrm{M}-\mathrm{N} \pi^{*}$ orbital of the other nitrido complex was proposed originally for $\left\{\mathrm{Os}^{\mathrm{V}} \equiv \mathrm{N}\right\}$ nitride coupling. ${ }^{[52,133]}$ Finally, formation of the mononuclear $\mathrm{N}_{2^{-}}$ adduct $7^{\mathrm{Me}}$ from the binuclear $\mathrm{N}_{2}$-bridged species $14^{\mathrm{Me}}$ and $\mathrm{N}_{2}$ is exergonic by another $3 \mathrm{kcal} \mathrm{mol}^{-1}$, according to DFT. ${ }^{8}$

\section{6. $\mathrm{C}-\mathrm{H}$ activation}

The partial 'nitridyl' character of $\mathbf{1 3}$ raises the question as to whether intermolecular radical $\mathrm{C}-\mathrm{H}$ activation is a viable reaction pathway. Hence, an excess of 1,4-cyclohexadiene (4.3 equiv.) was added to a sample of photochemically generated $\mathbf{1 3}$ and warmed to room temperature. However, as in the case without cyclohexadiene, the $\mathrm{N}_{2}$ complexes 14 and 7 were the only products detected by ${ }^{1} \mathrm{H}$ and ${ }^{31} \mathrm{P}$ NMR spectroscopy. Furthermore, no conversion of cyclohexadiene was observed. This result suggests that hydrogen atom abstraction is not kinetically competitive with $\mathrm{N}-\mathrm{N}$ coupling for $\mathbf{1 3}$ even with hydrocarbons

\footnotetext{
${ }^{8}$ DFT computations were performed by Prof. Dr. Bas de Bruin.
} 

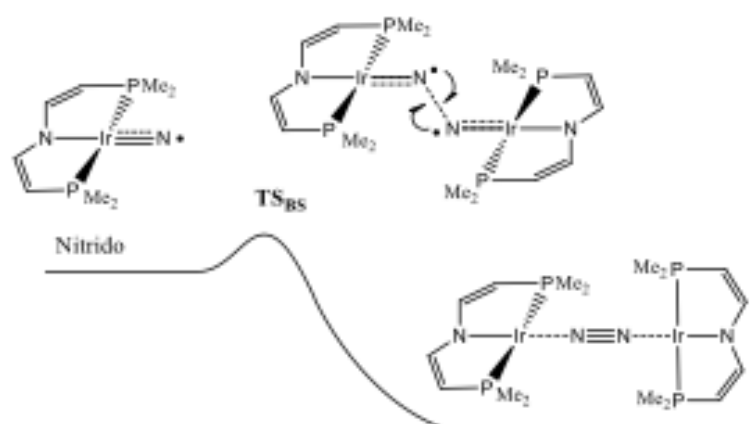

$\mathrm{N}_{2}$-bridged binuclear $\mathrm{Ir}^{\mathrm{t}}$ species

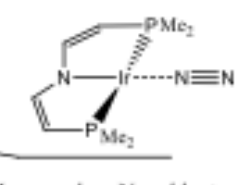

Mononuclear $\mathrm{N}_{2}$-adduct

Figure II.2.15.: Calculated energy barriers for formation of the $N_{2}$-bridged species $14^{\text {Me }}$ via bimolecular radical coupling of the neutral nitrido complex 13.

that form highly stabilized radical intermediates, such as 1,4-cyclohexadiene and will be discussed in detail in chapter 4. 


\section{Square-planar rhodium nitrido complex}

The stabilization of terminal square-planar nitrido complexes of Ir by the new PNP pincer ligand ${ }^{[200]}$ encouraged investigations on terminal nitrido complexes of Rh. The results of this chapter are published in 2013 under the title: „Synthesis and Reactivity of a Transient, Terminal Nitrido Complex of Rhodium“. ${ }^{[222]}$

\subsection{Square-planar rhodium(II) chloro and azido complexes}

The reaction of Wilkinson's complex $\left[\mathrm{RhCl}\left(\mathrm{PPh}_{3}\right)_{3}\right]$ with 1 and in situ oxidation with 2.5 equivalents of 1,4-benzoquinone affords rhodium(II) pincer complex $[\mathrm{RhCl}\{\mathrm{N}(\mathrm{CHCH}$ $\left.\left.\left.\mathrm{P}^{t} \mathrm{~B}_{2}\right)_{2}\right\}\right](\mathbf{1 5})$ as green solid in around $50 \%$ isolated yield, similar to the corresponding iridium(II) complex $\mathbf{3}$ (scheme II.3.5). ${ }^{[200]}$

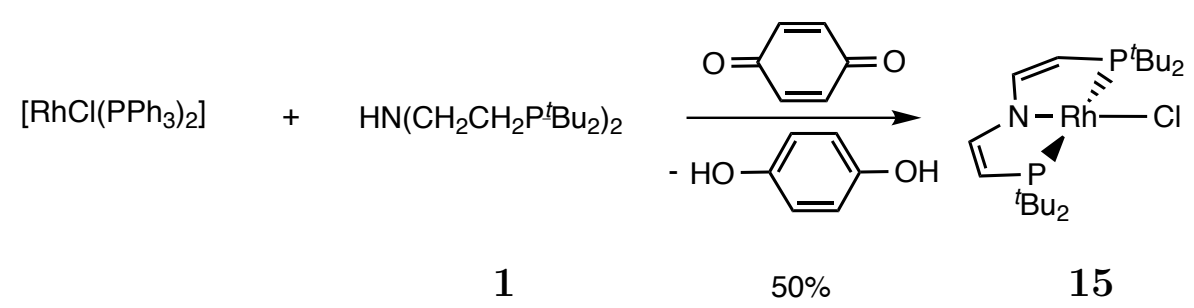

Scheme II.3.5: Conversion of the Wilkinson complex with the PNP pincer ligand and in situ oxidation to square-planar rhodium(II) chloro complex 15.

Removal of residual $\mathrm{PPh}_{3}$ from the crude product failed via extraction, crystallization and column chromatography, however only succeeded by sublimation $i$. vac. at $60{ }^{\circ} \mathrm{C}$ 


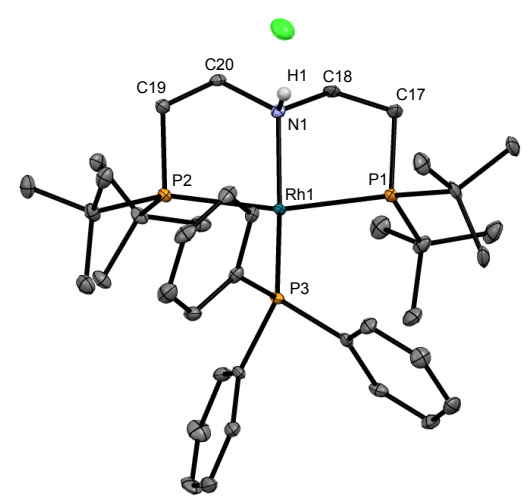

Figure II.3.1.: Molecular structure of $\left[\mathrm{Rh}\left(\mathrm{PPh}_{3}\right)\left\{\mathrm{HN}\left(\mathrm{CH}_{2} \mathrm{CH}_{2} \mathrm{P}^{\mathrm{t}} \mathrm{Bu} \mathrm{u}_{2}\right\}_{2}\right)\right] \mathrm{Cl}$ in the crystal (thermal ellipsoids at 50\% probability; hydrogen atoms except for $\mathrm{H} 1$ omitted for clarity.) Selected bond lengths [A] and angles $\left[^{\circ}\right]$ : Rh1-N1 2.170(3), Rh1-P3

2.2611(11), Rh1-P1 2.4356(10), Rh1-P2 2.3666(10), C17-C18 1.512(5), C19-C20

1.513(5), N1-C18 1.480(5), N1-C20 1.486(4), N1-H1 0.92(4); N1-Rh1-P3 164.09(9), P1-Rh1-P2 156.56(4).

for several hours. The mechanism for the formation of $\mathbf{1 5}$ was not examined. However, without an oxidizing agent, the $\mathrm{Rh}^{\mathrm{I}}$ complex $\left[\mathrm{Rh}\left(\mathrm{PPh}_{3}\right)\left\{\mathrm{HN}\left(\mathrm{CH}_{2} \mathrm{CH}_{2} \mathrm{P}^{t} \mathrm{Bu}_{2}\right)_{2}\right\}\right] \mathrm{Cl}$ was isolated and crystallographically characterized (figure II.3.1), probably representing an intermediate prior to backbone dehydrogenation. ${ }^{1}$

Salt metathesis of $\mathbf{1 5}$ with a mixture of $\left[\mathrm{N}\left(\mathrm{Ph}_{3}\right)_{2}\right] \mathrm{N}_{3} / \mathrm{NaN}_{3}$ (1:9) in acetone gives the azido complex $\left[\mathrm{Rh}\left(\mathrm{N}_{3}\right)\left\{\mathrm{N}\left(\mathrm{CHCHP}^{t} \mathrm{Bu}_{2}\right)_{2}\right\}\right]$ (16) in almost quantitative yield. Interestingly, the reaction time for full conversion of $\mathbf{1 5}$ is four times longer than for $\mathbf{3}$ under identical reaction conditions.

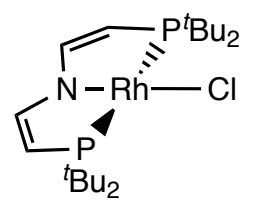

15

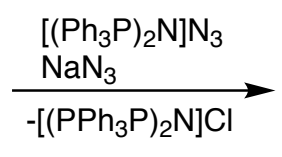

$89 \%$

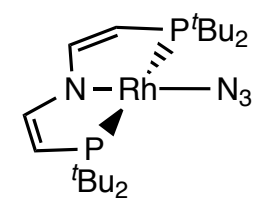

16

Scheme II.3.6: Synthesis of square-planar rhodium(II) azido complex 16.

\footnotetext{
${ }^{1}$ First investigations on the synthesis of $\mathbf{1 5}$ were performed by M. Sc. Yanlin Wu. ${ }^{[223]}$ Determination of the molecular structures within this project were performed in collaboration with Prof. Dr. Dietmar Stalke by M. Sc. Lennard Krause and Dr. Elena Karl.
} 
Two (15) and three (16) paramagnetically broadened and shifted signals in the ${ }^{1} \mathrm{H}$ NMR spectra, respectively, indicate $C_{2 V}$ symmetry on the NMR timescale. The missing backbone proton signal of $\mathbf{1 5}$ is assumed to be superimposed with the broad and intense signal of the ${ }^{t} \mathrm{Bu}$ group, as indicated by the peak integral. Note that these two signals are close in the case of complex $\mathbf{1 6}$ as well $(\Delta \delta=3.0 \mathrm{ppm})$. The magnetic moment of $\mathbf{1 5}$ ( $\mu_{\text {eff }}=1.7 \mu_{B}$, Evans's method) is in agreement with an $S=1 / 2$ ground state. The large anisotropy of the $g$-tensors $\left(\mathbf{1 5}: g_{11}=3.25, g_{22}=1.82, g_{33}=1.68 ; \mathbf{1 6}: g_{11}=2.92\right.$, $g_{22}=1.96, g_{33}=1.85$ ) in the rhombic EPR spectra (figure II.3.2) of $\mathbf{1 5}$ and $\mathbf{1 6}$ (figure II.3.2) indicate the presence of metal-centered $\left(\mathrm{Rh}^{\mathrm{II}}\right)$ radical complexes. ${ }^{2}$
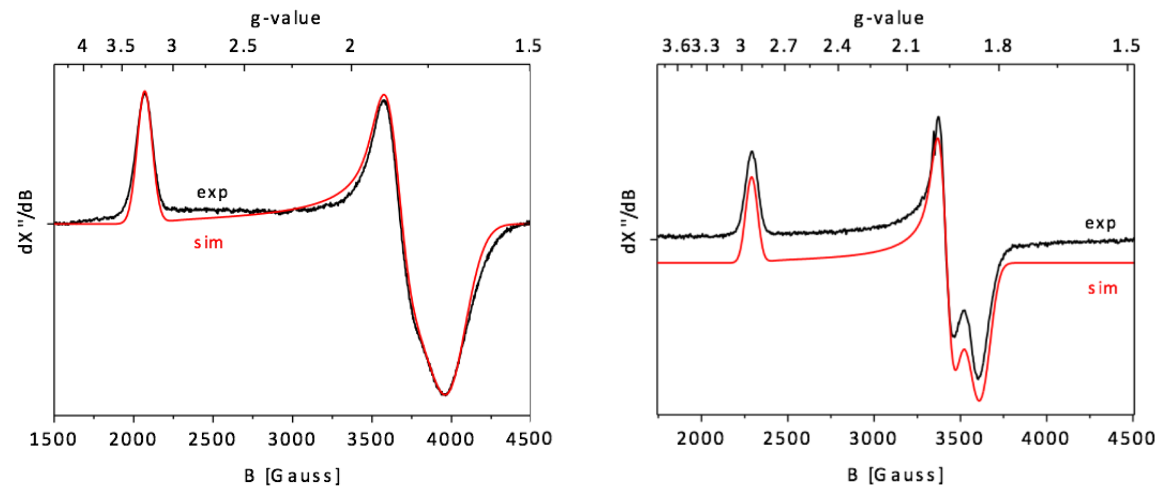

Figure II.3.2.: Experimental and simulated EPR spectra in frozen toluene of $\mathbf{1 5}$ ( $T=$ $117 \mathrm{~K} ; F_{\text {req }}=9.436340 \mathrm{GHz}$; modulation amplitude, $1 \mathrm{G}$; microwave power, $5.023 \mathrm{~mW})$ left and $16\left(\mathrm{~T}=20 \mathrm{~K} ; F_{\text {req }}=9.380845 \mathrm{GHz}\right.$; modulation amplitude, $4 \mathrm{G}$; microwave power, $0.2 \mathrm{~mW}$ ) right.

The azide stretching vibration of $\mathbf{1 6}$ was assigned to a strong peak at $2040 \mathrm{~cm}^{-1}$ in the IR spectrum. DFT electronic structure (b3lyp/def2-TZVP) and EPR (BP86/ZORA/TZVP) property calculations are in good agreement with the spectroscopic results $\left(15: g_{11}=\right.$ $\left.3.13, g_{22}=1.88, g_{33}=1.52 ; \mathbf{1 6}: g_{11}=2.79, g_{22}=1.94, g_{33}=1.79\right)$ and corroborate the assignments as rare examples of square-planar rhodium(II) complexes (Mulliken spin density on Rh: $81 \%(\mathbf{1 5}), 74 \%(\mathbf{1 6})) .{ }^{3[173-175,224]}$ The molecular structure of $\mathbf{1 5}$ was also 


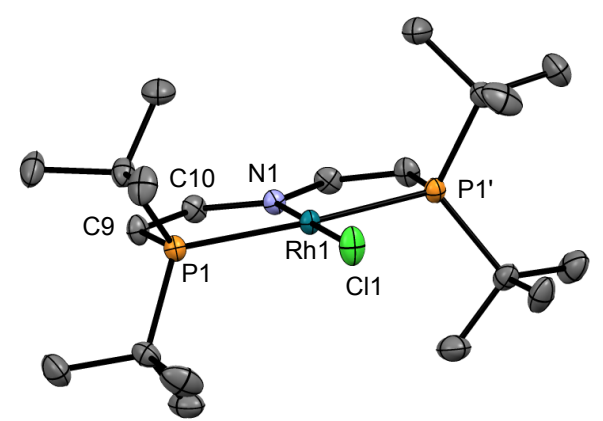

Figure II.3.3.: DIAMOND plot of the molecular structure of 15 from single-X-ray diffraction (ellipsoids set at $50 \%$ probability, hydrogen atoms are omitted for clarity). Selected bond lengths [A] and angles [\%: Rh1-Cl1 2.3344(7), Rh1-N1 1.9667(14), Rh1-P1 2.3249(7), C9-C10 1.3499(16), N1-Rh1-Cl1 180, P1-Rh1-P1' 166.573(16).

determined by single-crystal X-ray diffraction (figure II.3.3), confirming the square-planar coordination geometry around the metal.

\subsection{Open-shell rhodium nitride}

Irradiation of $\mathbf{1 6}$ in frozen toluene at $20 \mathrm{~K}$ led to the gradual disappearance of its EPR signal and clean formation of exclusively one new signal (scheme II.3.7, figure II.3.4). ${ }^{4}$

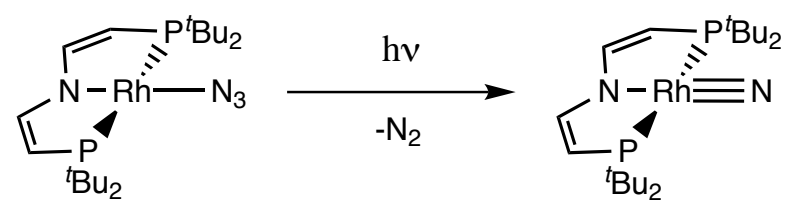

Scheme II.3.7: Synthesis of open-shell rhodium nitrido complex 17.

All components of the rhombic $g$-tensor $\left(g_{11}=2.04, g_{22}=1.93, g_{33}=1.70\right)$ are close to or well below the value for the free electron $\left(g_{e}=2.002\right)$. This new signal was assigned

\footnotetext{
${ }^{2}$ EPR spectroscopy of $\mathbf{1 5}$ was performed in collaboration with Dr. A. Claudia Stückl. EPR spectroscopy of $\mathbf{1 6}$ and all simulations were performed by Prof. Dr. Bas de Bruin.

${ }^{3}$ DFT calculations were performed by Prof. Dr. Bas de Bruin.

${ }^{4}$ EPR spectroscopy, simulations and DFT were performed by Prof. Dr. Bas de Bruin.
} 
to rhodium(IV) nitride $\left[\mathrm{Rh}(\mathrm{N})\left\{\mathrm{N}\left(\mathrm{CHCHP}^{t} \mathrm{Bu}_{2}\right)_{2}\right\}\right]$ (17). The EPR spectrum of $\mathbf{1 7}$ is similar to that of iridium(IV) nitrido complex $\mathbf{1 3}\left(g_{11}=1.89, g_{22}=1.63, g_{33}=1.32\right)$, for which the unusual $g$-tensor was rationalized on the basis of extensive mixing via spinorbit coupling of the nearly degenerate SOMO and LUMO, which exhibit strong $\mathrm{Ir} \equiv \mathrm{N}$ $\pi^{*}$-MO character. ${ }^{[200]}$ The smaller orbital contributions to the $g$-tensor of $\mathbf{1 7}$, as comparison with 13, are in agreement with the smaller spin-orbit coupling (SOC) constant of Rh. ${ }^{[225]}$ In contrast to $\mathbf{1 3}$, hyperfine interactions (HFI) are resolved along one direction of the HFI tensor. The clearly resolved ${ }^{14} \mathrm{~N}$ hyperfine coupling $\left(A_{22}=+65 \mathrm{MHz}\right)$ is close to the value found for 13 by Davies ENDOR spectroscopy $\left(A_{22}=+64 \mathrm{MHz}\right),{ }^{, 200]}$ further indicating similar electronic structures of 13 and 17. Spectral simulations and line shape analysis point to a smaller ${ }^{103} \mathrm{Rh}$ hyperfine coupling of $\sim 25 \mathrm{MHz}$ along $g_{22}$.

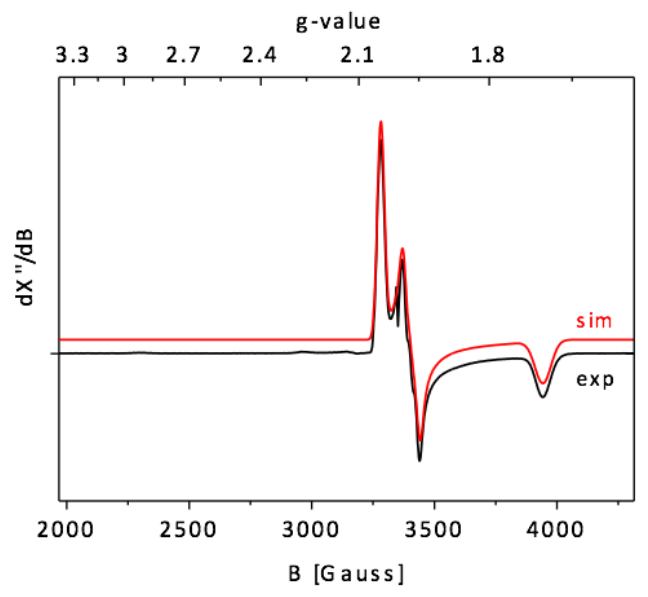

Figure II.3.4.: Experimental and simulated EPR spectra of rhodium nitrido species 17 obtained by irradiation of 16 with $U V$ light in frozen toluene $\left(T=20 \mathrm{~K} ; F_{\text {req }}=\right.$ $9.380845 \mathrm{GHz}$; modulation amplitude, $4 \mathrm{G}$; microwave power, $0.2 \mathrm{~mW}$ ).

The formation of a nitrido complex is further supported by vibrational spectroscopy. Short irradiation of a $\mathrm{KBr}$ pellet of $\mathbf{1 6}$ causes the appearance of a weak band at $874 \mathrm{~cm}^{-1}$ in the IR spectrum (figure II.3.6 top), which was assigned to the $\mathrm{Rh} \equiv \mathrm{N}$ stretching vibration of 17 . The band disappears on extended irradiation. Upon use of partially ${ }^{15} \mathrm{~N}$-azide labeled 16, a second band at $848 \mathrm{~cm}^{-1}$ was also observed (figure II.3.6 bottom) and matches well with the expectations derived from the harmonic oscillator approximation for the ${ }^{\mathbf{1 5} / \mathbf{1 4}} \mathrm{N}-\mathbf{1 7}$ isotopologues $\left(\Delta \tilde{\nu}=26 \mathrm{~cm}^{-1}\right)$. These values are also in excellent agreement with the DFT computer stretching frequencies for $\mathbf{1 7}\left({ }^{14} \mathrm{~N}: 871 \mathrm{~cm}^{-1}\right.$; 
${ }^{15} \mathrm{~N}$ : $842 \mathrm{~cm}^{-1}$ ) upon applying the same scaling factor as was used for $\mathbf{1 2}$ and $\mathbf{1 3}$. Notably, the $\mathrm{M} \equiv \mathrm{N}$ stretching vibration of $\mathbf{1 7}$ is slightly lower than that in the analogous Ir complex $13\left(901 \mathrm{~cm}^{-1}\right)$. Hence, the ratio of the harmonic oscillator force constants $\left(\frac{f_{R h}}{f_{I r}}=\frac{\nu_{R h}^{2} \mu_{R h}}{\nu_{I r}^{2} \mu_{I r}}=0.88\right)$ indicates weaker $\mathrm{Rh} \equiv \mathrm{N}$ than $\mathrm{Ir} \equiv \mathrm{N}$ bonding. In agreement, DFT calculations reveal a weaker $\mathrm{M} \equiv \mathrm{N}$ Mayer bond order for Rh compared to that for Ir (Rh: 1.972; Ir: 2.290), and also formation of the nitrido species from its azido precursor is calculated to be less exergonic $\left(\Delta G_{298 K}^{0} \mathrm{Rh}:-4.7 \mathrm{kcal} / \mathrm{mol}\right.$; Ir: $\left.-14.3 \mathrm{kcal} / \mathrm{mol}\right)$ and has a higher kinetic barrier $\left(\Delta G_{298 K}^{\ddagger} \mathrm{Rh}:+33.4 \mathrm{kcal} / \mathrm{mol}\right.$; Ir: $\left.+27.8 \mathrm{kcal} / \mathrm{mol}\right)$.

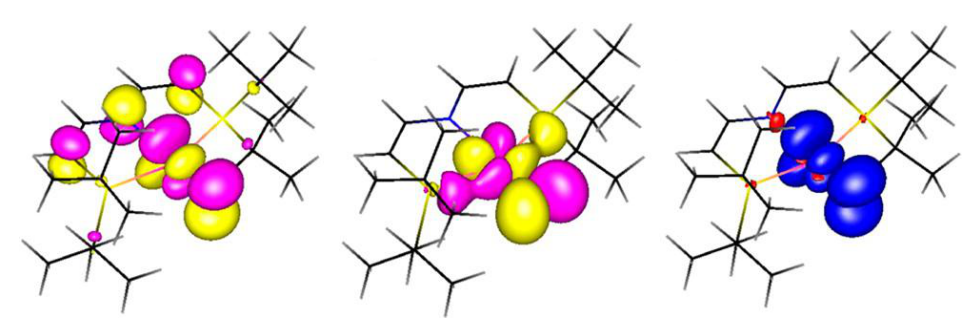

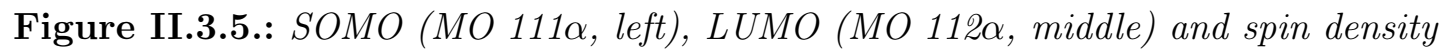
plot (right) of $\mathbf{1 7}$.

The EPR spectroscopic assignments were also substantiated computationally. According to DFT, the electronic structure of $\mathbf{1 7}$ is analogous to that of $\mathbf{1 3}$. The SOMO (MO $111 \alpha$, figure II.3.5 left) represents an anti-bonding interaction between the metal $\left(d_{x y}\right)$ and the nitrido ligand $\left(p_{x}\right)$. As for $\mathbf{1 3}$, the other predominantly $\mathrm{M}-\mathrm{N} \pi^{*}$ anti-bonding orbital is close in energy (LUMO: MO $112 \alpha$, figure II.3.5 middle) and exhibits the appropriate (rotational) symmetry for efficient SOMO/LUMO excited state admixture of orbital angular momentum into the ground state via SOC, indicating minor effects of spin polarization on the distribution. Hence, the unpaired electron is mainly situated in a $\pi^{*} \mathrm{Rh}-\mathrm{N}$ orbital. As a consequence of the high degree of covalency within the $\mathrm{Rh} \equiv \mathrm{N}$ $\pi$-bonding, the spin density is strongly delocalized over this moiety (figure II.3.5 right), exhibiting a preference for the $\mathrm{N}(\sim 64 \%)$ over the $\mathrm{Rh}(\sim 37 \%)$ atom according to Muliken spin-densities (b3-lyp, def2-TZVP). Hence, the nitridyl-radical character of Rh complex $\mathbf{1 7}$ is even higher (and better defined due to smaller spin orbit coupling contributions) than for Ir complex $13\left(\sim 50 \%\right.$ spin density at N). ${ }^{[200]}$ The experimental $g$-tensor $\left(g_{11}=\right.$ 2.04, $\left.g_{22}=1.97, g_{33}=1.70\right)$ and the resolved ${ }^{14} \mathrm{~N}$ HFI value along $g_{22}\left(A_{22}=65 \mathrm{MHz}\right)$ 

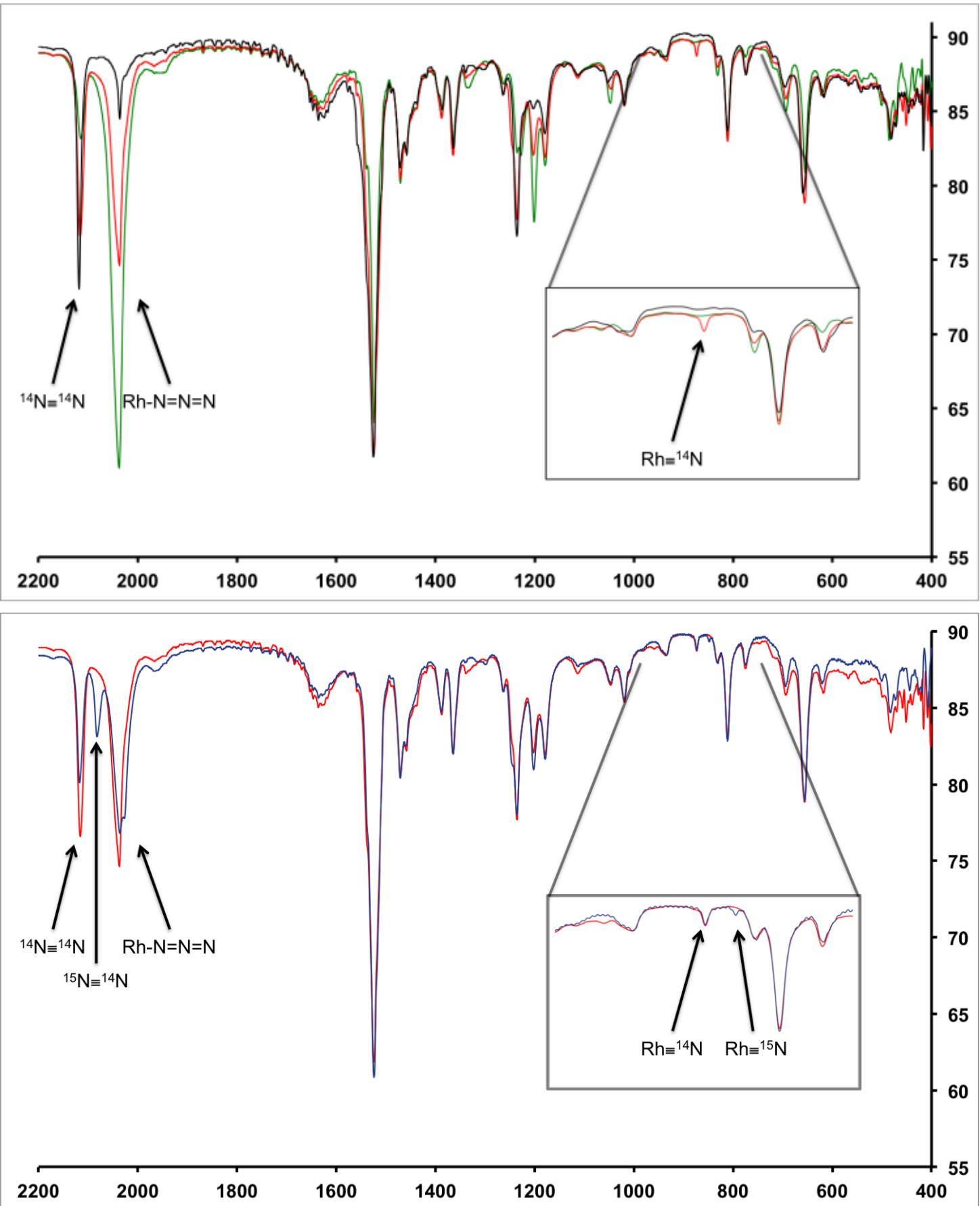

Figure II.3.6.: Top: Infrared spectra of $\mathbf{1 6}$ as $\mathrm{KBr}$ disc (green curve), directly after irradiation for $10 \mathrm{~s}$ from each side (red curve) and after 2 min of irradiation (black curve). Bottom: Irradiation of $\mathrm{KBr}$ disc for $10 \mathrm{~s}$ from each side of $\mathbf{1 6}$ (red curve) and $15 / 14 N-16$ (blue curve), respectively. 
of $\mathbf{1 7}$ are well reproduced by DFT property computations $\left(g_{11}=2.02, g_{22}=1.93, g_{33}=\right.$ $1.62 ; A_{22}^{N}=52 \mathrm{MHz}$ ), thus giving confidence in the calculated electronic structure.

\subsection{Reactivity of open-shell rhodium nitrido complex}

Upon thawing a frozen solution of $\mathbf{1 7}$, the EPR signature disappears immediately. Two diamagnetic molecules are formed after photolysis in frozen solution of in the liquid phase at $-60{ }^{\circ} \mathrm{C}$. Their ratio is subject to the reaction conditions: Irradiation in solution under an atmosphere of dinitrogen produces the $\mathrm{Rh}^{\mathrm{I}}$ complex $\left[\mathrm{Rh}\left(\mathrm{N}_{2}\right)\left\{\mathrm{N}\left(\mathrm{CHCHP}^{t} \mathrm{Bu}_{22}\right\}\right](\mathbf{1 8})\right.$, selectively, which was fully characterized including single-crystal X-ray diffraction (figure II.3.7)..$^{5}$

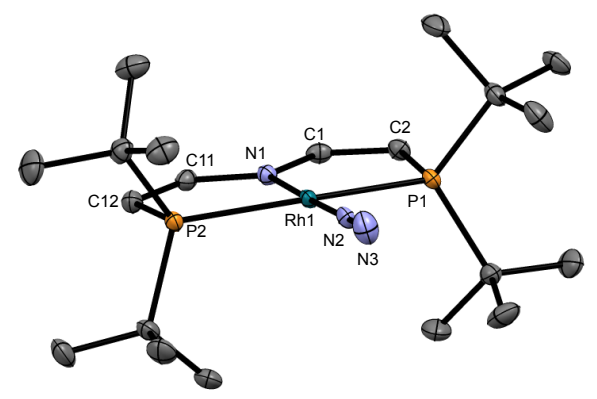

Figure II.3.7.: DIAMOND plot of the molecular structure of 18 from single-X-ray diffraction (ellipsoids set at 50\% probability, hydrogen atoms are omitted for clarity). Selected bond lengths $[A]$ and angles $\left[^{\circ}\right]$ : Rh1-N1 2.025(3), Rh1-N2 1.911(3), Rh1-P1 2.3246(10), Rh1-P2 2.3165(10), N2-N3 1.091(4), C1-C2 1.351(5), C11-C12 1.354(5); N1-Rh-N2 17r.93(11), P1-Rh1-P2 164.12(3).

The IR spectrum of $\mathbf{1 8}$ exhibits an intense band at $2120 \mathrm{~cm}^{-1}$ that can be assigned to the $\mathrm{N}-\mathrm{N}$ stretching vibration and is shifted to $2083 \mathrm{~cm}^{-1}$ when generation of 18 starting from ${ }^{\mathbf{1 5} / \mathbf{1 4}} \mathbf{N}^{\mathbf{1 4}} \mathbf{N}_{\mathbf{2}} \mathbf{- 1 6}$ (harmonic oscillator: $\Delta \tilde{\nu}=36 \mathrm{~cm}^{-1}$ ). NMR characterization

\footnotetext{
${ }^{5}$ Determination of the molecular structure of $\mathbf{1 8}$ was performed by Prof. Dr. Dietmar Stalke and M. Sc. Elena Carl.
} 
$\left({ }^{31} \mathrm{P}: \delta=78 \mathrm{ppm}\right)$ is in agreement with $C_{2 V}$ symmetry in solution. However, irradiation of $\mathbf{1 6}$ in solution under vacuum results in partial formation of $\mathbf{1 8}(\sim 34 \%)$. The isolation of the main product $(66 \%)$ was unsuccessful, owing to very similar solubility, but NMR ( $\left.{ }^{31} \mathrm{P}: \delta=75 \mathrm{ppm}\right)$ and electrospray mass spectrometry (ESI-MS) characterization (figure II.3.8 top) are in agreement with the assignment to bridging complex $\left[\left(\mathrm{Rh}\left\{\mathrm{N}\left(\mathrm{CHCHP}^{t} \mathrm{Bu}_{2}\right)_{2}\right\}\right)_{2}\left(\mathrm{~N}_{2}\right)\right](\mathbf{1 9})$. The use of partially ${ }^{15} \mathrm{~N}$-labeled 16 enabled the detection of the $\mathrm{N}_{2}$ ligand by ${ }^{15} \mathrm{~N}$ NMR spectroscopy $\left(\delta=-76 \mathrm{ppm}\right.$ vs. $\left.\mathrm{Me}^{15} \mathrm{NO}_{2}\right)$ and of all three possible isotopomers $\left({ }^{15} \mathrm{~N}^{15} \mathrm{~N}-\mathbf{1 9},{ }^{15} \mathrm{~N}^{14} \mathrm{~N}-\mathbf{1 9},{ }^{14} \mathrm{~N}^{14} \mathrm{~N}-19\right)$ by ESI-MS (figure II.3.8 bottom), confirming that the azide group is the source for $\mathrm{N}_{2}$ formation. ${ }^{6}$

These results are in agreement with coupling of the transient nitride $\mathbf{1 7}$ to a bridging $\mathrm{N}_{2}$ complex (19), which dissociates to the terminal $\mathrm{N}_{2}$ complex 18 in the presence of excess $\mathrm{N}_{2}$, e.g. from azide splitting (scheme II.3.8). In contrast to the previously reported $\mathrm{Ir}^{\mathrm{IV}}$ nitride 13, the corresponding $\mathrm{Rh}^{\mathrm{IV}}$ nitride $\mathbf{1 7}$ is too reactive to be detected in the liquid phase. It is tempting to attribute the lower thermal stability of $\mathbf{1 7}$ to the enhanced $N$-radical character. In agreement with these observations, the DFT calculated barrier for N-N coupling is lower for rhodium $\left(\Delta G_{289 K}^{\ddagger}=+11.9 \mathrm{kcal} / \mathrm{mol}\right)$ than for iridium $\left(\Delta G_{289 K}^{\ddagger}=+13.7 \mathrm{kcal} / \mathrm{mol}\right)$.

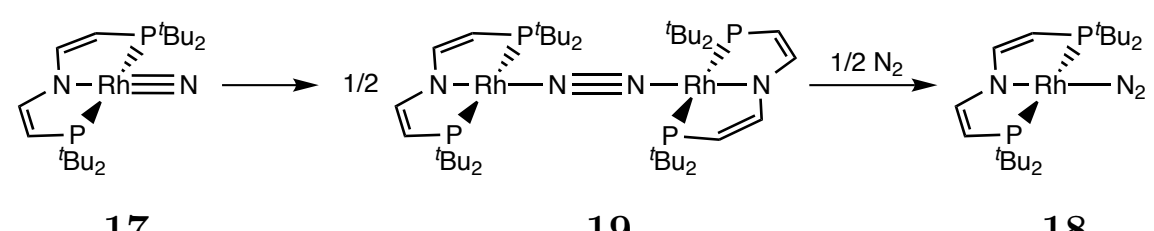

Scheme II.3.8: Decay of nitride $\mathbf{1 7}$ under the formation of $\mathbf{1 9}$ and dimer cleavage under formation of monomeric 18.

Interestingly, irradiation of $\mathbf{1 6}$ in the presence of 10 equiv. of 1,4-cyclohexadiene $\left(\mathrm{BDE}_{\mathrm{C}-\mathrm{H}}\right.$ $=+76 \mathrm{kcal} / \mathrm{mol})^{[226]}$ gave the same product $(\mathbf{1 8})$ as without the hydrogen donor reagent (DFT estimated $\mathrm{BDE}_{\mathrm{N}-\mathrm{H}}$ of $\left.\left[\mathrm{Rh}(\mathrm{NH})\left(\mathrm{N}\left\{\mathrm{CHCHPMe}_{2}\right\}_{2}\right)\right]:+78.7 \mathrm{kcal} / \mathrm{mol}\right)$. In good agreement, DFT calculations reveal a substantially higher kinetic barrier for HAT from 1,4-cyclohexadiene to $\left[\mathrm{Rh}(\mathrm{NH})\left(\mathrm{N}\{\mathrm{CHCHP} \mathrm{Me}\}_{2}\right)\right]\left(\Delta G_{289 K}^{\ddagger}=+21.9 \mathrm{kcal} / \mathrm{mol}\right)$ as compared to the barrier for $\mathrm{N}-\mathrm{N}$ coupling of two nitridyl radical species to form $[(\mathrm{Rh}(\mathrm{N}\{\mathrm{CHCH}$ $\left.\left.\left.\left.\mathrm{PMe}_{2}\right\}_{2}\right)\right)_{2}\left(\mathrm{~N}_{2}\right)\right]\left(\Delta G_{289 K}^{\ddagger}=+11.9 \mathrm{kcal} / \mathrm{mol}\right)$.

\footnotetext{
${ }^{6}$ Mass spectrometry was performed in collaboration with M. Sc. Krisitan Dalle.
} 

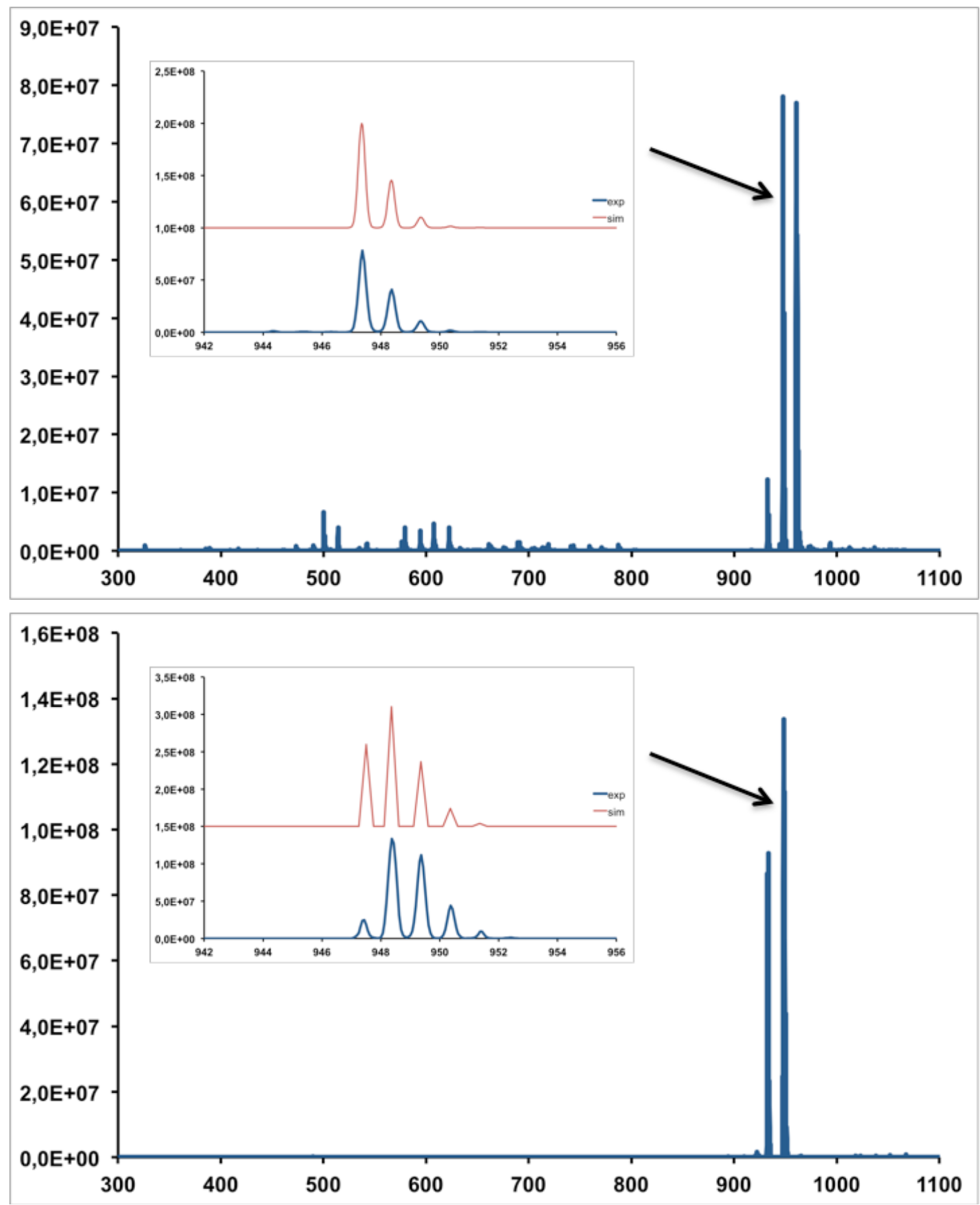

Figure II.3.8.: Experimental (blue) and simulated (red) ESI ${ }^{+}$Mass spectra of 19 (top) and ${ }^{15} \mathrm{~N}-19$ (below) in toluene. 


\section{Series of parent Ir amino, amido, imido and nitrido complexes}

Nitrogen containing molecules are ubiquitous in organic chemistry and despite few examples, the nitrogen source is commonly derived from $\mathrm{NH}_{3}$ synthesized by Nitrogenase activity $^{[6]}$ or by the industrial Haber-Bosch process ${ }^{[5]}$. Examples for direct incorporation of $\mathrm{N}_{2}$ within the synthesis of organic compounds i.e. via functionalization of metal nitrido complexes derived from $\mathrm{N}_{2}$ are rare, due to the intrinsic stability of the $\mathrm{M} \equiv \mathrm{N}$ moiety and hence, strong electrophiles are usually required for nitride transfer. ${ }^{[24,84,103,227]}$ Direct catalytic intramolecular $s p^{3} \mathrm{C}-\mathrm{H}$ amination reactions gained increasing interest, e.g. for heterocycle formation, ${ }^{[228-230]}$ and became powerful tools in total synthesis $^{[231-233]}$. The central intermediate proposed for transition metal catalyzed $\mathrm{C}-\mathrm{H}$ aminations are metal nitrene (or imido) species, ${ }^{[228,234-236]}$ depending on their respective electronic structures and are derived from low valent metal centers such as ruthenium(II) porphyrins ${ }^{[234,236,237]}$ or rhodium(I) paddlewheel complexes. The nitrene transfer reagents are commonly generated in situ by oxidation of tosylamine with iodosobenzene. Insertion of the transition metal nitrene into the $\mathrm{C}-\mathrm{H}$ bond occurs either via concerted $\mathrm{C}-\mathrm{H}$ insertion ${ }^{[229]}$ or through $\mathrm{C}-\mathrm{H}$ abstraction and subsequent radical rebound ${ }^{[234,235,237-239]}$. However, C-H amination reactions catalyzed by metal imido/nitrene complexes are limited to primary amines substituted with electronic withdrawing substituents such as tosylates and sulfamate esters. ${ }^{[230]}$ A general rationalization of an (electronic) structure reactivity relationship for insertions of nitrenes in $\mathrm{C}-\mathrm{H}$ bonds remains elusive.

$\mathrm{C}-\mathrm{H}$ insertions of terminal nitrido complexes, in contrast, are limited to stoichiometric intramolecular reactions and performed by electrophilic late-transition $5 d$ metal nitrides ${ }^{[89,90]}$, or via radical reaction pathways by nitrides coordinated to $4 d^{[89,186,187,240,241]}$ and $3 d^{[91]}$ transition metals or to uranium ${ }^{[185,242]}$. Remarkably, highly transient open- 
shell nitrido complexes of $\operatorname{Ir}(\mathbf{1 3})^{[200]}$ and $\mathrm{Rh}(\mathbf{1 7})^{[222]}$ decompose solely via selective $\mathrm{N}-\mathrm{N}$ coupling reaction towards dinitrogen bridged dimeric complexes, even in the presence of weak $\mathrm{C}-\mathrm{H}$ bonds e.g. 1,4-cyclohexadiene $\left(\mathrm{BDE}_{C-H}(\mathrm{gas})=76.0 \mathrm{kcal} / \mathrm{mol}\right)$, and $\mathrm{H}$ atom transfer (HAT) or $\mathrm{C}-\mathrm{N}$ coupling was not observed.

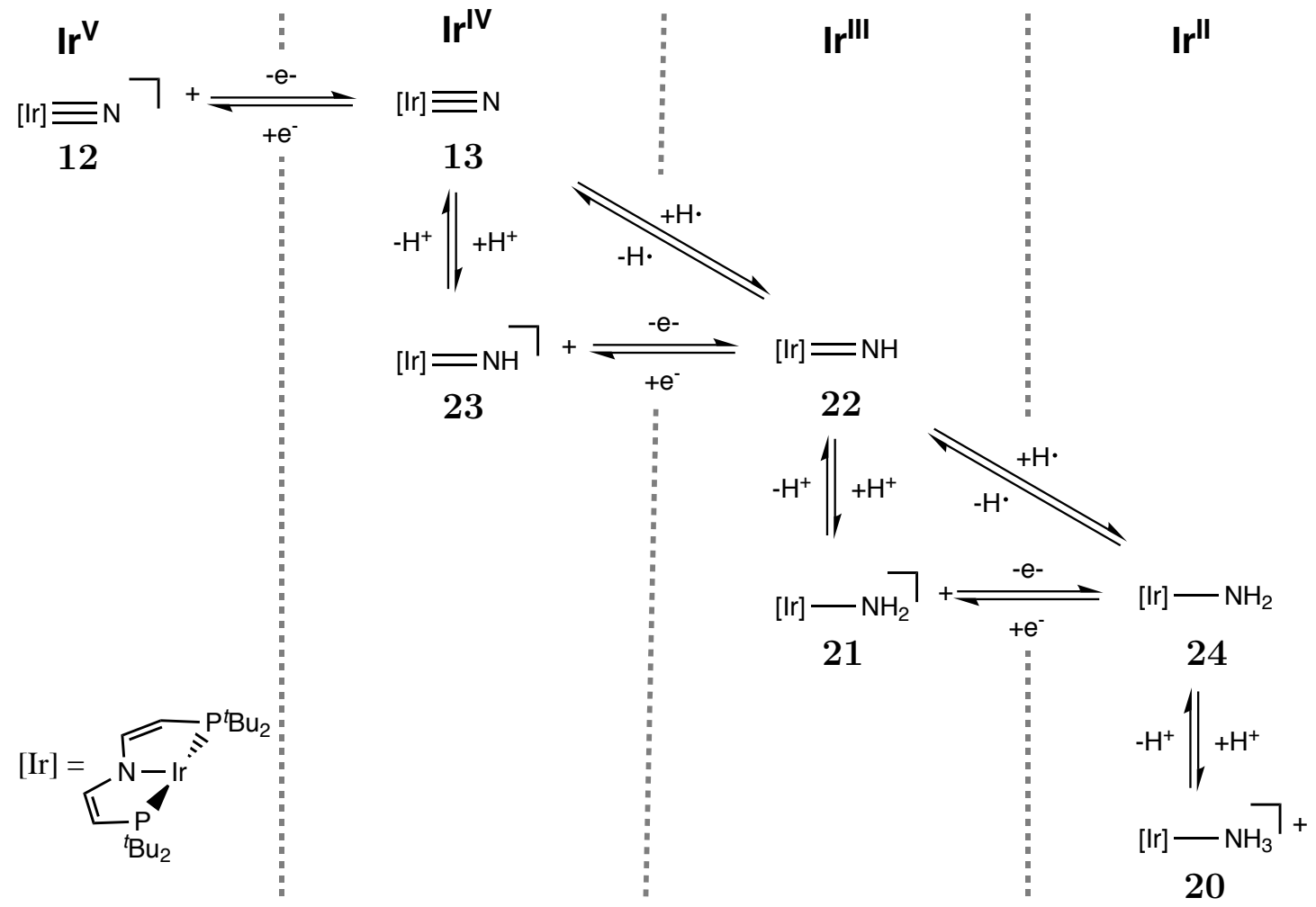

Chart II.4.1: Interconversion of a full series of Ir-NH complexes $(x=0$-3) by either successive proton and electron transfer, or via proton coupled electron transfer. The Ir exhibits formal oxidation states ranging from II to $V$, stabilized by a PNP pincer platform.

In this chapter, interconversion of nitrido complexes of Ir $(\mathbf{1 2 , 1 3})$ by sequential proton and electron transfer steps or HAT will provide a series of parent imido $(\mathbf{2 2 , 2 3})$, amido $(\mathbf{2 4 , 2 1})$ and amino (20) complexes (chart II.4.1). Estimation of the $\mathrm{N}-\mathrm{H}$ bond dissociation energies (BDE) of these complexes will be used to rationalize the selectivity for $\mathrm{N}-\mathrm{N}$ coupling reaction of Ir nitrido complex 13 over $\mathrm{E}-\mathrm{H}$ activation ( $\mathrm{E}=$ element). 


\subsection{Parent iridium(II-III) amido and amino complexes}

Stirring 3 in the presence of sodium amide (excess) in THF results in the slow, yet selective formation of parent amido complex 24 as monitored by ${ }^{1} \mathrm{H}$ NMR spectroscopy (scheme II.4.1).

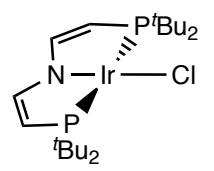

3

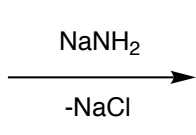

$90 \%$

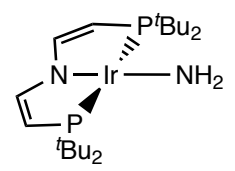

24

Scheme II.4.1: Synthesis of parent iridium amido complex 24.

The slow conversion of starting material is attributed to the poor solubility of sodium amide and thus accelerated by addition of one equivalent of 15-crown-5. After $2 \mathrm{~h}$, full conversion is achieved and green $\mathbf{2 4}$ can be isolated in analytically pure form after removal of all inorganic salts by filtration over celite in $90 \%$ yield. The ${ }^{1} \mathrm{H}$ NMR spectrum of 24 (figure II.4.1) exhibits four paramagnetically broadened and shifted signals at chemical shifts of $\delta=5.9\left({ }^{t} \mathrm{Bu}\right),-33.1\left(\mathrm{NH}_{2}\right),-53.9(\mathrm{CH})$ and $-73.1 \mathrm{ppm}(\mathrm{CH})$, indicating $C_{2 V}$ symmetry on the NMR timescale. The assignment of signal at $\delta=-33.2 \mathrm{ppm}$ to the $\mathrm{NH}_{2}$ protons is based on the relative intensity of the low intense signals.

The magnetic moment of $\mathbf{2 4}\left(\mu_{e f f}=1.82 \mu_{B}\right.$, Evans' method) is in agreement with a $\mathrm{S}$ $=1 / 2$ ground state, however, significantly lower than in chloro complex $\mathbf{3}$. In analogy to starting material $\mathbf{3},{ }^{[163]}$ no signal is observed in the ${ }^{31} \mathrm{P}$ NMR spectrum of $\mathbf{2 4}$. 


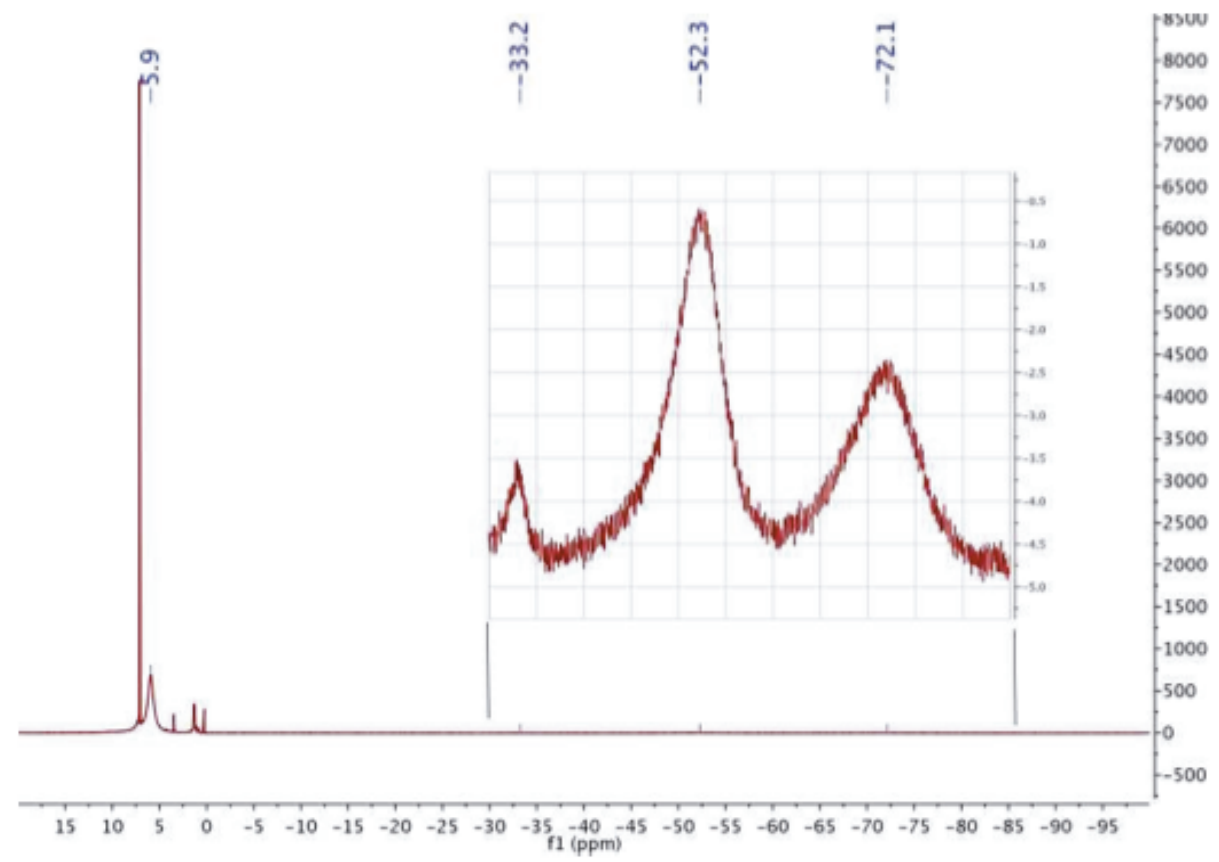

Figure II.4.1.: ${ }^{1} H$ NMR spectrum of 24 in $C_{6} D_{6}$ at room temperature.

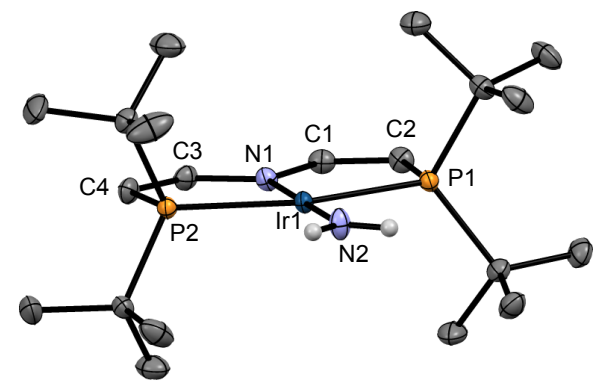

Figure II.4.2.: ORTEP plot of the molecular structure of 24 (carbon bound hydrogen atoms are omitted for clarity). Selected bond lengths $[A]$ and angles $[\%$ : Ir $1-N 1$ 2.0194(15), Ir1-N2 1.9521(17), Ir1-P1 2.3116(5), Ir1-P2 2.3086(5), N1-C1 1.380(2), N1-C3 1.385(2), C1-C2 1.348(3), C3-C4 1.344(3); N1-Ir1-N2 179.74(7), P1-Ir1-P2 164.445(17). 
Suitable crystals for determination of the molecular structure in the solid state via Xray diffraction were obtained by slow evaporation of a pentanes solution of $24{ }^{1}$ The molecular structure of $\mathbf{2 4}$ (figure II.4.2) confirms square-planar coordination around the metal center $\left(\Sigma_{\text {angles }}=359.98^{\circ}\right)$ with a linear $\mathrm{N}_{\mathrm{PNP}}-\mathrm{Ir}-\mathrm{NH}_{2}$ axis $\left(179.74(7)^{\circ}\right)$. The sum of angles around the $\mathrm{NH}_{2}$-nitrogen $\left(\Sigma_{\text {angles }}=360^{\circ}\right)$ indicates $\mathrm{sp}^{2}$ hybridization and the $\mathrm{Ir}-\mathrm{NH}_{2}$ distance of $1.9521(17) \AA$ compares well with other parent iridium amido complexes in square-planar coordination geometry $\left(\left[\operatorname{Ir}(\mathrm{PDI})\left(\mathrm{NH}_{2}\right)\right]: \quad 1.926(15) \AA^{[28]}\right.$; $\left[\operatorname{Ir}(\mathrm{PPEP})\left(\mathrm{NH}_{2}\right)\right]:$ 1.954(4) $\AA^{[243]}$, figure II.4.3), however, is significantly shorter than in a coordination number of five $\left(\left[\operatorname{Ir}\left(\mathrm{PCP}^{*}\right)(\mathrm{H})\left(\mathrm{NH}_{2}\right)\right]: 1.999(4) \AA\right)^{[244]}$ or $\operatorname{six}([\operatorname{Ir}(\mathrm{PCP})$ $\left.\left.\left(\mathrm{NC}^{t} \mathrm{Bu}\right)(\mathrm{H})\left(\mathrm{NH}_{2}\right)\right]: 2.193(4) \AA^{[245]} ;\left[\operatorname{Ir}\left(\mathrm{C}_{5} \mathrm{Me}_{5}\right)\left(\mathrm{PMe}_{3}\right)(\mathrm{Ph})\left(\mathrm{NH}_{2}\right)\right]: 2.105(8) \AA^{[246]}\right)$ bearing hydrocarbyl-ligands in trans-position. The structural features within the pincer backbone are not affected by the exchange of chloride (3) vs. amide (24), as expressed by the similar C-N (24: 1.380(2) and 1.385(2) $\AA ;$ 3: 1.387(2) $\AA)$ and $\mathrm{C}-\mathrm{C}(\mathbf{2 4}$ : 1.348(3) and

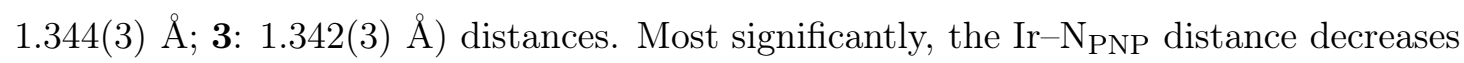
from 24 to 3 (24: 2.0194(15); 3: 1.985(2) $\AA^{[163]}$ ) due to the stronger trans-influence of the amido ligand.

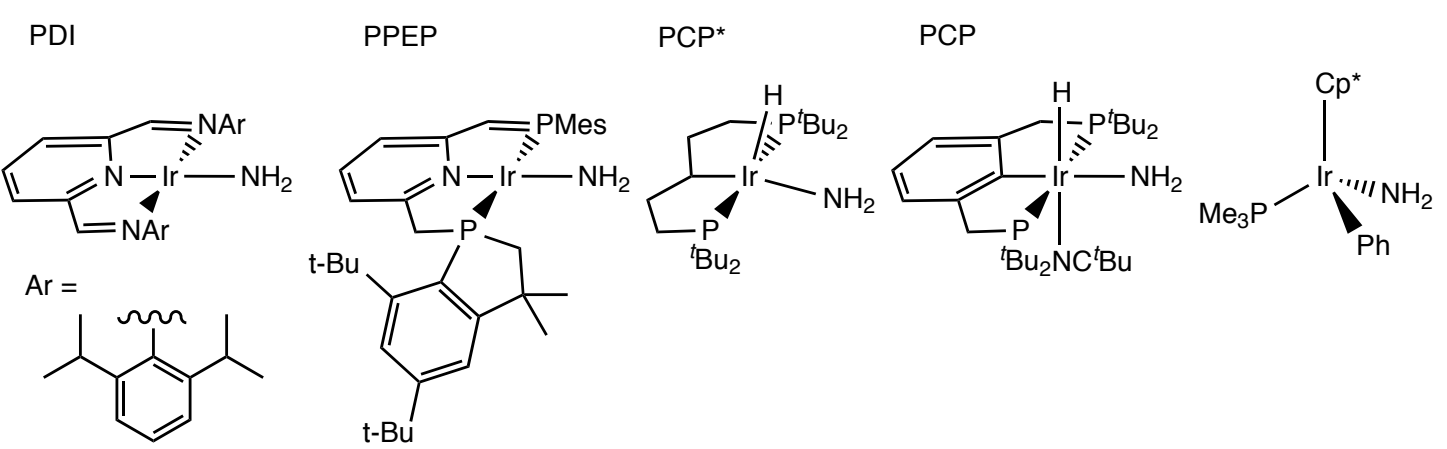

Figure II.4.3.: Structurally characterized monomeric parent amido complexes of iridium.

\footnotetext{
${ }^{1}$ Determination of the molecular structure of $\mathbf{2 4}$ was performed by Dr. Frank W. Heinemann.
} 
Exposition of a solution of $\mathbf{2 4}$ to oxygen or air results in fast and selective formation of 10 in an isolated yield of $35 \%$ in analytically pure form (scheme II.4.2).

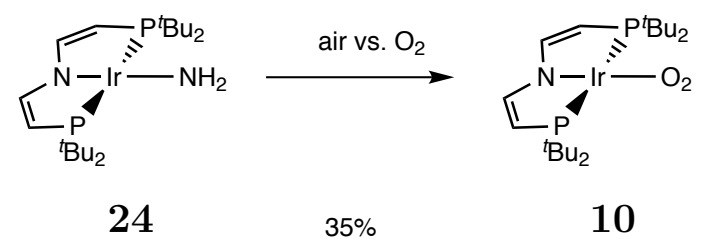

Scheme II.4.2: Reactivity of amido complex 24 with air or oxygen.

24 reacts readily with $\mathrm{HCl}$ under formation of $\mathbf{3}$ and $\mathrm{NH}_{3}$, as confirmed by ${ }^{1} \mathrm{H}$ NMR spectroscopy. Protonation of $\mathbf{2 4}$ at the amido ligand towards an $\mathrm{Ir}^{\mathrm{II}}$ amino complex and ligand exchange vs. chloride is assumed and in agreement with the basic reactivity of coordinated $\mathrm{NH}_{2}$ ligands. ${ }^{[247]}$

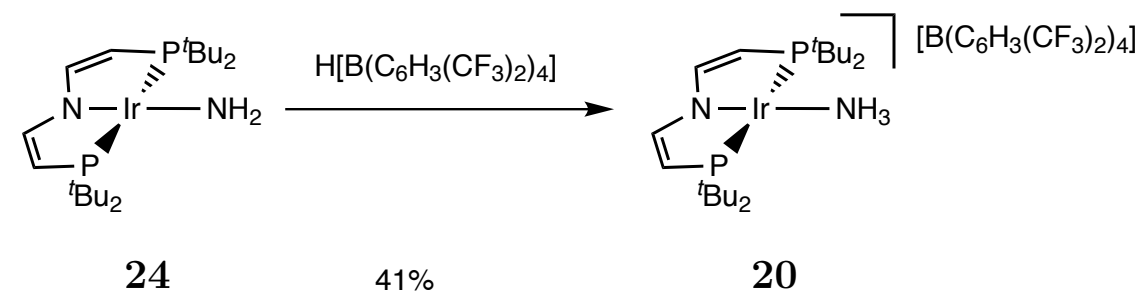

Scheme II.4.3: Protonation of 24 to Ir ${ }^{I I}$ amino complex 20.

The utilization of the less nucleophilic acid HBarf $\left(\mathrm{Barf}=\left[\mathrm{B}\left(\mathrm{C}_{6} \mathrm{H}_{3}\left(\mathrm{CF}_{3}\right)_{2}\right)_{4}\right]^{-}\right)$allows for the isolation of cationic $\mathrm{Ir}^{\mathrm{II}}$ amino complex 20 in $41 \%$ isolated yield (scheme II.4.3). Besides two narrow singlets at $\delta=7.79$ and $7.56 \mathrm{ppm}$ attributable to the aromatic protons of the counter ion $\left[\mathrm{B}\left(\mathrm{C}_{6} \mathrm{H}_{3}\left(\mathrm{CF}_{3}\right)_{2}\right)_{4}\right]^{-}$, only two paramagnetically broadened and shifted signals at $\delta=38.4$ and $13.5 \mathrm{ppm}$ are observable in the ${ }^{1} \mathrm{H}$ NMR spectrum of $\mathbf{2 0}$ (figure II.4.4). The signal at higher field is assigned to the tert-butyl groups, due to the higher signal intensity. No further signals were found in a range of -160 and $130 \mathrm{ppm}^{2}$

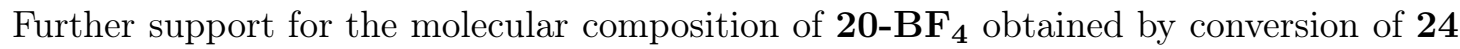

\footnotetext{
${ }^{2}$ Synthesis of $\mathbf{2 0}$ was performed by M. Sc. Markus Kinauer.
} 


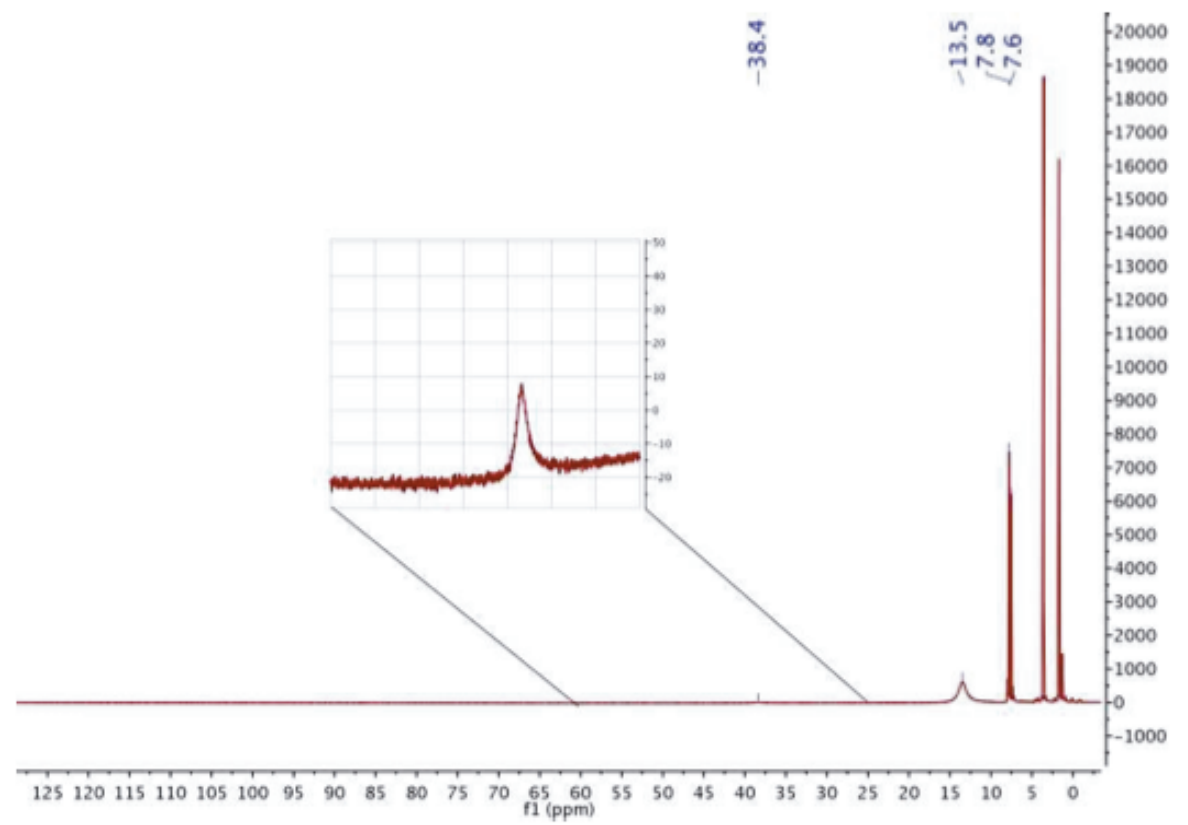

Figure II.4.4.: ${ }^{1} H$ NMR spectrum of 20 in $d_{8}-T H F$ at room temperature.

with $\mathrm{HBF}_{4}$ was provided by single crystal X-Ray diffraction. ${ }^{3}$ The molecular structure

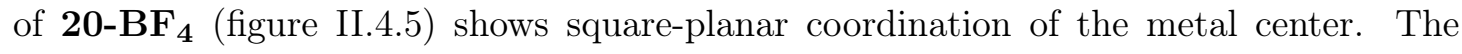
residual electron density around N2 suggested three further hydrogen substituents and hence, supports the presence of an $\mathrm{NH}_{3}$ group as fourth ligand at Ir1. However, residual electron density close to the metal center above the $\operatorname{Ir}(\mathrm{PNP})$ plane does not allow for detailed discussion of bond lengths and angles.

The cyclic voltammogram of $\mathbf{2 4}$ (figure II.4.6) exhibits one reversible oxidation wave at $\mathrm{E}_{1 / 2}=-0.68 \mathrm{~V}$ and one irreversible reduction process at a cathodic peak potential of $\mathrm{E}_{\mathrm{pc}}=-2.60 \mathrm{~V}$ (vs. $\mathrm{Fc}^{0 /+}$ ). The lower oxidation potential of the Ir ${ }^{\mathrm{II} / \mathrm{III}}$ redox couple for amido complex $\mathbf{2 4}$ in comparison to chloride $\mathbf{3}$ is attributed to the stronger $\pi$-donor properties of amide and in alignment with the trends within the Ir- $\mathrm{N}_{\mathrm{PNP}}$ bond lengths of both complexes.

Remarkably, the oxidation of $\mathrm{Ir}^{\mathrm{IV}}$ nitrido complexes $\mathbf{1 3}\left(\mathrm{E}_{1 / 2}=-0.8 \mathrm{~V}\right)^{[200]}$ proceeds at even lower potential than of $\mathrm{Ir}^{\mathrm{II}}$ amido complex $\mathbf{2 4}$ and is counterintuitive with respect to the higher formal metals oxidation state of $\mathbf{1 3}$ as compared with $\mathbf{2 4}$. However, the high degree of covalency within the $\mathrm{Ir}-\mathrm{NH}_{\mathrm{x}}$ multiple bonds $(\mathrm{x}=0-2)$ obscures the as-

\footnotetext{
${ }^{3}$ Determination of the molecular structure of $\mathbf{2 0 -} \mathbf{B F}_{\mathbf{4}}$ as performed by Dr. Christian Würtele.
} 


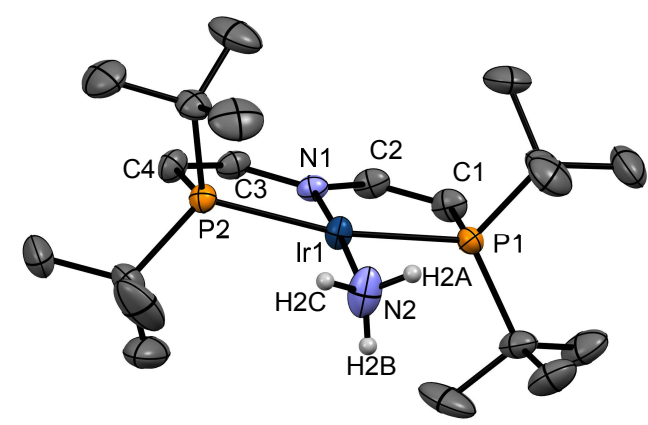

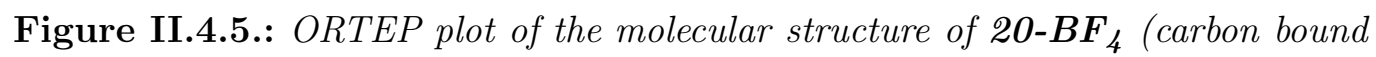
hydrogen atoms, one molecule of THF and counterion $B_{4}$ are omitted for clarity). Selected bond lengths [A] and angles [\%: Ir1-N1 1.988(3), Ir1-N2 2.210(4), Ir1-P1 2.3263(10), Ir1-P2 2.3300(11), N1-C2 1.386(5), N1-C3 1.376(5), C1-C2 1.340(6), C3-C4 1.340(6); N1-Ir1-N2 174.42(15), P1-Ir1-P2 164.35(4).

signment of formal oxidation states solely based on strict charge separation depending on the relative electronegativities of the respective bonding partners. Instead, the lower oxidation potential of $\mathbf{1 3}$ is attributed to a higher SOMO energy as compared with $\mathbf{2 4}$ and is in alignment with the transient character of $\mathbf{1 3 .}$

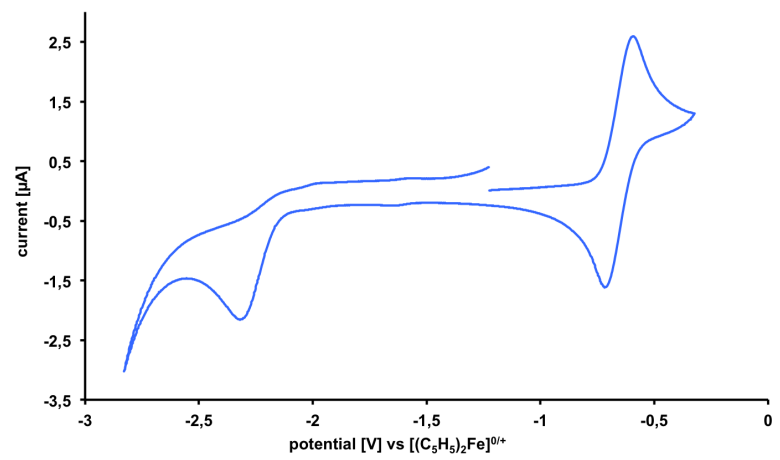

Figure II.4.6.: Cyclic voltammogram of $24\left(10^{-3} \mathrm{~mol} / \mathrm{L}\right)$ in $\mathrm{THF}(400 \mathrm{mV} / \mathrm{s}$, $\left[\mathrm{nBu} u_{4} \mathrm{~N}_{\mathrm{PF}} 0.1 \mathrm{~mol} / \mathrm{L}\right.$, Pt working electrode, Pt counter electrode, Pt pseudo reference electrode).

Chemical oxidation of $\mathbf{2 4}$ using $\mathrm{AgPF}_{6}$ yields in diamagnetic cationic parent amido complex 21 (scheme II.4.4). Residual starting material is removed by extraction with non- 
polar solvents to accomplish the isolation of purple $\mathbf{2 1}$ in analytically pure form after crystallization from $\mathrm{THF} /$ pentanes in $70 \%$ yield.

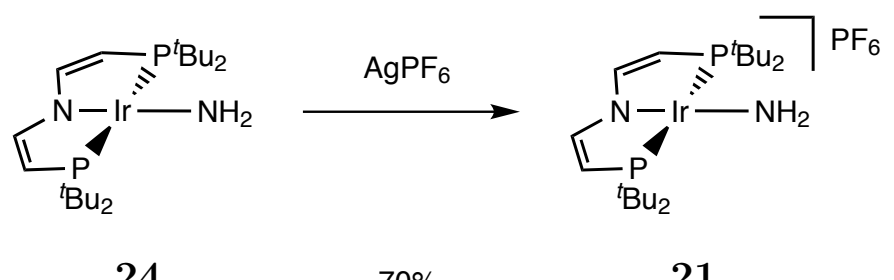

24

$70 \%$

21

Scheme II.4.4: Oxidation of 24 to closed-shell Ir ${ }^{I I I}$ amino complex 21.

The ${ }^{1} \mathrm{H},{ }^{13} \mathrm{C}$ and ${ }^{31} \mathrm{P}$ NMR spectra of $\mathbf{2 1}$ are in agreement with $C_{2 V}$ symmetry on the NMR timescale. The ${ }^{1} \mathrm{H}$ NMR chemical shift assigned to the $\mathrm{NH}_{2}$-group at $\delta=11.35 \mathrm{ppm}$ exhibits a significant low-field shift compared with other diamagnetic parent iridium amido complexes $\left(\left[\operatorname{Ir}(\mathrm{PDI})\left(\mathrm{NH}_{2}\right)\right]: 5.99 \mathrm{ppm}^{[28]} ;\left[\operatorname{Ir}(\mathrm{PPEP})\left(\mathrm{NH}_{2}\right)\right]: 3.82 \mathrm{ppm}^{[243]}\right.$; [Ir $\left.\left(\mathrm{PCP}^{\prime}\right)(\mathrm{H})\left(\mathrm{NH}_{2}\right)\right]: 4.24 \mathrm{ppm}^{[244]} ;\left[\mathrm{Ir}\left(\mathrm{Cp}^{*}\right)\left(\mathrm{PMe}_{3}\right)(\mathrm{Ph})\left(\mathrm{NH}_{2}\right)\right]$ : -1.32 ppm $\left.{ }^{[246]}\right)$. Remarkably, the chemical shift of the signal assignable to the pincer backbone protons in $\alpha$ position to the phosphine is higher than for the protons in $\alpha$-position to the amide and is in contrast to the NMR properties of all reported diamagnetic iridium and rhodium complexes with the fully dehydrogenated PNP pincer ligand.[163,193,200,222,248]

Suitable crystals for X-ray diffraction experiments of $\mathbf{2 1}$ were obtained by oxidation of 24 with $\mathrm{AgSbF}_{6} \cdot \mathbf{2 1}_{\mathbf{- S b F}} \mathbf{6}$ (figure II.4.7) exhibits a $C_{2 V}$ symmetric molecular structure in the solid state with a square-planar coordinated metal center $\left(\Sigma_{\text {angles }}=360^{\circ}\right)$ and a basically linear $\mathrm{N}_{\mathrm{PNP}}-\mathrm{Ir}-\mathrm{NH}_{2}$ axis $\left(179.07(10)^{\circ}\right)$. The structural features within the PNP ligand of square-planar $[\operatorname{Ir}(\mathrm{PNP}) \mathrm{X}]^{+}\left(\mathrm{X}=\mathrm{Cl}, \mathrm{NH}_{2}\right)$ are not affected by the nature of the X ligand, as expressed by identical Ir- $\mathrm{N}_{\mathrm{PNP}}(\mathbf{4}: 1.922(2) \AA ; 21: 1.926(2) \AA), \mathrm{N}-\mathrm{C}$

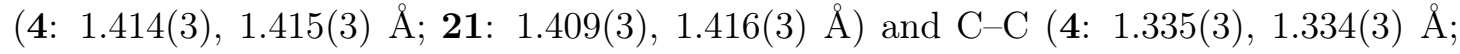
21: $1.335(3), 1.334(3) \AA$ ) bond lengths, ${ }^{[163]}$ pointing towards similarities in their electronic structures. This is also demonstrated by shorter Ir- $\mathrm{N}_{\mathrm{PNP}}(\mathbf{2 4}: 2.0194(15) \AA ; 21$ : 1.926(2) $\AA)$ and $\operatorname{Ir}-\mathrm{NH}_{2}(24: 1.9521(17) \AA ; 21: 1.900(2) \AA)$ distances of closed-shell vs. open-shell parent amido complexes, which can be rationalized by stronger $\pi$-donation of both amido ligands into vacant $\pi$-symmetric $d$-orbitals in the case of $\mathbf{2 1}$.

Examples of square-planar metal complexes with a $d^{6}$ electronic structure are rare. ${ }^{[249]}$ These strongly coordinatively unsaturated systems tend to exhibit an intermediate-spin electronic structure as reported for $\left[\mathrm{M}(\mathrm{X})\left\{\mathrm{N}\left(\mathrm{SiMe}_{2} \mathrm{CH}_{2} \mathrm{P}^{t} \mathrm{Bu}_{2}\right)_{2}\right\}\right](\mathrm{M}=\mathrm{Os}, \mathrm{Ru} ; \mathrm{X}=\mathrm{F}$, 


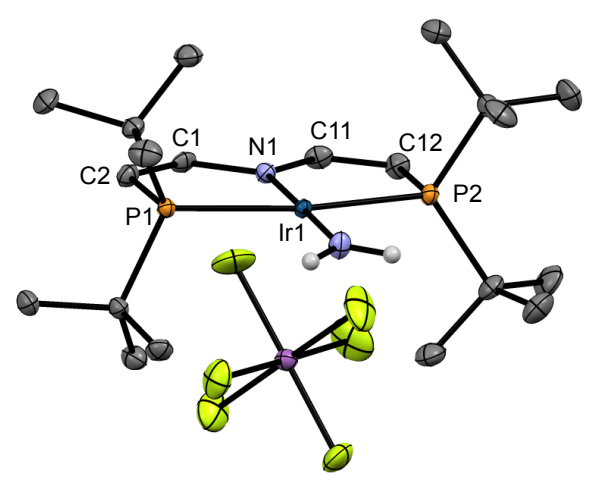

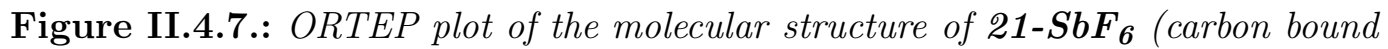
hydrogen atoms are omitted for clarity). Selected bond lengths $[\AA]$ and angles $[\%$ : Ir1-N1 1.926(2), Ir1-N2 1.900(2), Ir1-P1 2.3333(8), Ir1-P2 2.3351(8), N1-C1 1.409(3), N1-C11 1.416(3), C1-C2 1.331(4), C11-C12 1.329(4); N1-Ir1-N2 179.07(10), P1-Ir1-P2 166.42(2).

$\mathrm{Cl}$, I, OTf $)^{[150,151,250]}$ or a low-spin configuration as demonstrated for $\left[\mathrm{RuCl}\left\{\mathrm{N}\left(\mathrm{CH}_{2} \mathrm{CH}_{2}\right.\right.\right.$ $\left.\left.\left.\mathrm{P}^{t} \mathrm{Bu}_{2}\right)_{2}\right\}\right]$ (IX) with the stronger $\pi$-donating ligand ${ }^{[140]}$. In comparison to the latter, the low spin configuration of $\mathbf{4}$ is attributed to the positive charge of the complex and the change from $4 d$ to $5 d$ metal. ${ }^{[163]}$ Remarkably, the isolated square-planar chloro complexes of $\mathrm{Ru}$ and Ir with $d^{6}$ electronic configuration possessing low spin state are thermally not robust and decompose at ambient temperatures. ${ }^{[140,163]}$ In contrast, 21 is stable at room temperature even in solution over a prolonged time. This thermal robustness in particular by comparison with highly temperature sensitive chloro complex 4 is attributed to the amido ligand. Stabilization might arise by stronger $\pi$-donation of the amide to the metal center. Note that thermally stable square-planar chloro complex $\left[\operatorname{Ir}\left\{\mathrm{C}\left(\mathrm{CH}_{2} \mathrm{CH}_{2} \mathrm{P}^{t} \mathrm{Bu}_{2}\right)_{2}\right\}(\mathrm{Cl})\right]$ is isoelectronic to 4 and is reported with a low-spin state. ${ }^{[251]}$ However, the electronic structure assigned in between the formulations of an $\operatorname{Ir}^{\mathrm{I}}$ carbene complex (structure A, figure II.4.8) and $\mathrm{Ir}^{\mathrm{III}}$ ylid (structure B, figure II.4.8) obscures the assignment of oxidation states for the metal. ${ }^{[252]}$

\subsection{Iridium imido complexes}

Fully characterized terminal parent imido complexes are rare ${ }^{[253]}$ and reports about stable examples are limited to transition metals of group $4^{[254-256]}, 5^{[257-260]}$ and $6^{[261-267]}$. 


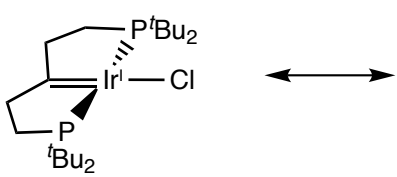

A

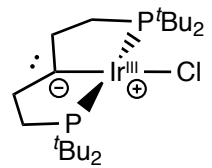

$\mathrm{B}$

Figure II.4.8.: Mesomeric structures of square-planar iridium chloro complex described as $\operatorname{Ir}^{I}$ carbene complex (A) or Ir III ylide complex (B).

The structurally characterized examples of $\mathrm{Os}^{[268,269]}$, however, are not stable in solution: The NH moiety of the cationic imido complex $\left[\mathrm{Os}\left(\mathrm{C}_{5} \mathrm{H}_{5}\right)\left(\mathrm{CH}_{2} \mathrm{SiMe}_{3}\right)_{2}(\mathrm{NH})\right]$ reported by Shapley and coworkers exhibits acidic reactivity and forms a mixture of $\mathrm{SiMe}_{4}$ and insoluble osmium-containing products at ambient temperatures. ${ }^{[268]}$ The second example of a parent osmium imido complex $\left[\mathrm{Os}(\mathrm{Tp})(\mathrm{Cl})_{2}(\mathrm{NH})\right]\left(\mathrm{Tp}=\right.$ tris-(pyrazolyl)borate) $^{[269]}$ was characterized crystallographically by Meyer and coworkers, however, is not observed in solution due to fast disproportionation. ${ }^{[130]}$ Furthermore, disproportionation of parent Fe imido complex $[\mathrm{Fe}(\mathrm{PDI})(\mathrm{NH})]\left(\mathrm{PDI}=2,6-\left(2,6-{ }^{i} \mathrm{Pr}_{2} \mathrm{C}_{6} \mathrm{H}_{3} \mathrm{NCMe}\right)_{2} \mathrm{C}_{5} \mathrm{H}_{3} \mathrm{~N}\right)$ as transient unobservable reaction intermediate upon conversion of $\left[\mathrm{Fe}(\mathrm{PDI})\left(\mathrm{N}_{2}\right)_{2}\right]$ with $\mathrm{H}$-dbabh $($ dbabh $=2,3: 5,6$-dibenzo-7-azabicyclo[2.2.1]hepta-2,5-diene) was postulated by Meyer, Chirik and coworkers. ${ }^{[270]}$

The synthesis of parent iridium imido complex $\mathbf{2 2}$ is envisioned by different synthetic strategies (scheme II.4.5): a) By abstraction of one $\mathrm{H}$ atom of neutral parent amido complex 24, b) by single HAT to neutral nitrido complex $\mathbf{1 3}$ and c) by deprotonation of neutral cationic amido complex 21.

The first synthetic approach towards parent imido complex $\mathbf{2 2}$ is HAT reaction with excess of an $\mathrm{H}$-atom donor reagent and neutral nitrido complex 13 (scheme II.4.5). ${ }^{4}$ Addition of excess TEMPO-H (= 1-Hydroxy-2,2,6,6-tetramethyloxylpiperidine) to a solution of $\mathbf{1 3}$ generated by irradiation of $\mathbf{1 1}$ at $-60{ }^{\circ} \mathrm{C}$ leads to quantitative formation of $\mathbf{2 4}$ and full conversion of starting material upon time of mixing (figure II.4.9 top). In contrast, nitride coupling of $\mathbf{1 3}$ is not affected by the presence of 2,4,6-tri-tert-butylphenol (figure II.4.9 middle), demonstrating the dependence of the reaction on the respective hydrogen donor reagent. ${ }^{5}$

\footnotetext{
${ }^{4}$ Note that bimolecular nitride coupling of $\mathbf{1 3}$ proceeds at room temperature and traces of coupling products $\mathbf{1 4}$ and $\mathbf{7}$ can not be prevented.

${ }^{5}$ One yet unidentified weak signal appeared at a chemical shift of $\delta=5.88 \mathrm{ppm}$, that could not be assigned to any diamagnetic Ir containing complex due to the absence of any unknown signals in the corresponding ${ }^{31} \mathrm{P}$ NMR spectrum.
} 

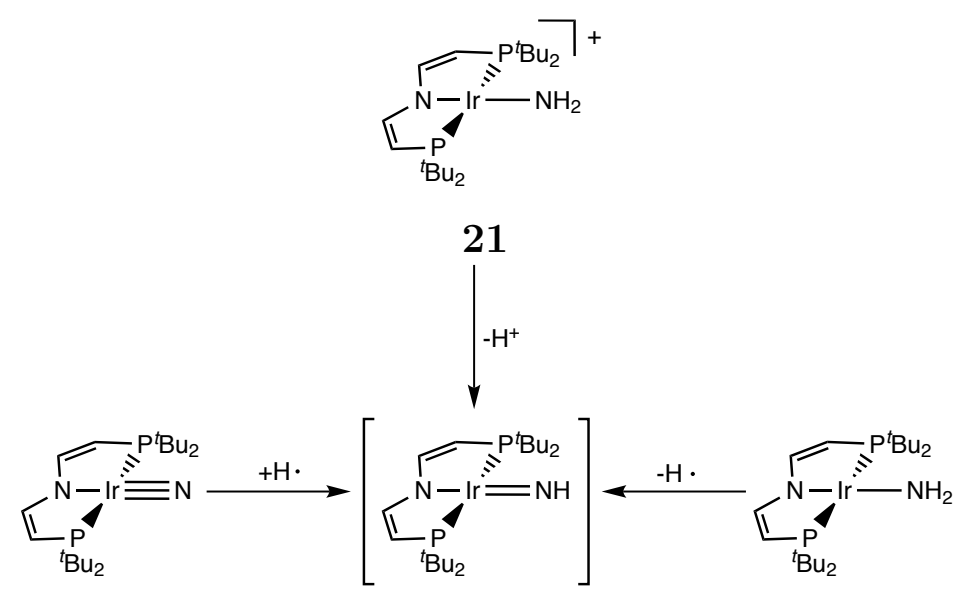

13

22

24

Scheme II.4.5: Synthetic strategies for parent Ir imido complex 22 by HAT reaction to neutral nitrido complex 13, by hydrogen atom abstraction from neutral amido complex 24 or by deprotonation of cationic amido complex 21.

Remarkably, only half conversion of $\mathbf{1 3}$ is observed upon addition of one equivalent of TEMPO-H and a product mixture consistent of a 1:1 mixture of $\mathbf{1 3}$ and $\mathbf{2 4}$ is obtained (figure II.4.10, top and scheme II.4.6 bottom). Additional trace amounts of dinitrogen complexes $\mathbf{7}$ and $\mathbf{1 4}$ are attributed to slow nitride coupling of $\mathbf{1 3}$ under the experimental conditions. However, one further reaction product exhibiting two broad signals in the ${ }^{1} \mathrm{H}$ NMR spectrum $(\delta=48.6$ and $4.3 \mathrm{ppm}$ ) could not be assigned to any Ir complex yet. Performing the same reaction in $d_{8}$-toluene as solvent, in contrast, results in full conversion of starting $\mathbf{1 3}$ and formation of $\mathbf{2 4}$ with trace amounts of nitride coupling products (figure II.4.10 bottom). The reverse reaction was investigated upon dissolving a mixture of amide $\mathbf{2 4}$ and one equivalent of 2,4,6-tri-tert-butylphenoxyl radical in $d_{8}$-toluene. A product mixture consistent of amide $\mathbf{2 4}(30 \%)$, nitride $\mathbf{1 3}(20 \%)$ and dinitrogen complexes $\mathbf{1 4}$ and $\mathbf{7}$ (20\% in total) is obtained (spectroscopic yields, figure II.4.11). 

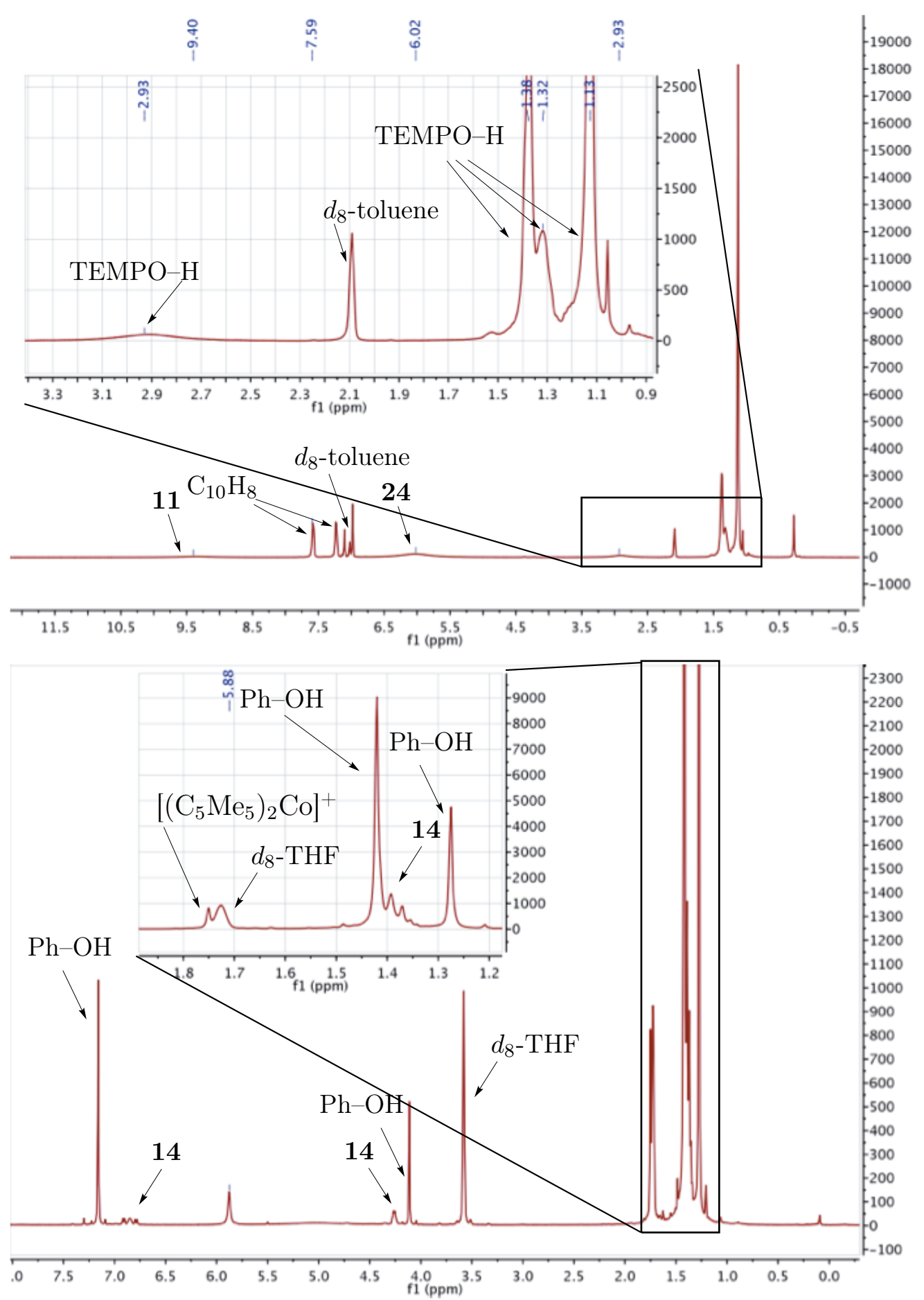

Figure II.4.9.: Top: Room temperature ${ }^{1} H$ NMR spectrum in $d_{8}$-toluene of a mixture of freshly prepared 13 by photolysis of 11 and subsequent conversion with excess of TEMPO-H. Bottom: ${ }^{1} H$ NMR spectrum in $d_{8}$-THF of a mixture consistent of Ph-OH, 12 and $\left[\left(\mathrm{C}_{5} \mathrm{Me}_{5}\right)_{2} \mathrm{Co}\right]$ at room temperature (Ph-OH = 2,3,6-tri-tert-butylphenol). 


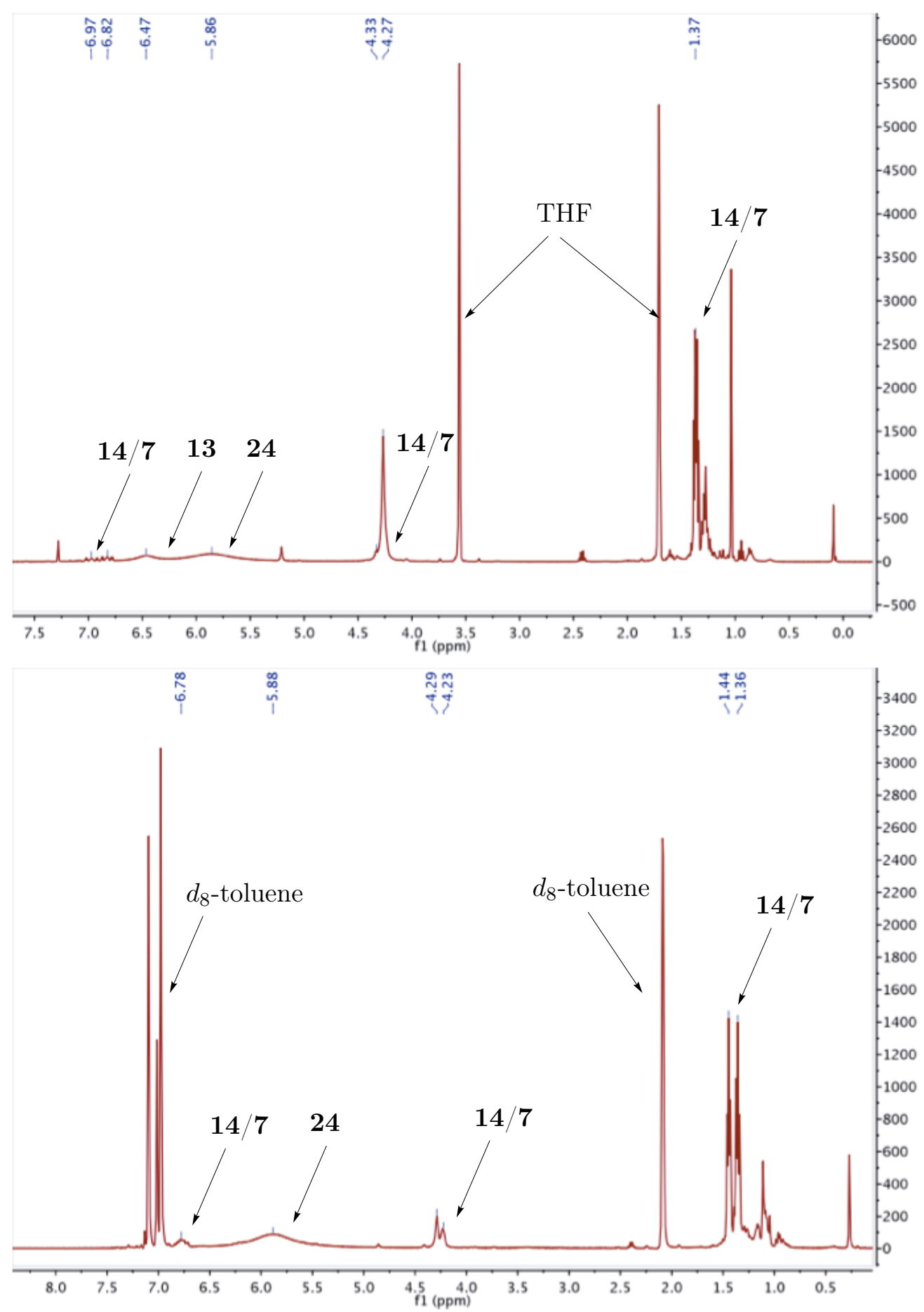

Figure II.4.10.: Room temperature ${ }^{1} H$ NMR spectrum of a stoichiometric mixture of 13 and TEMPO-H at room temperature in $d_{8}$-THF (top) and in $d_{8}$-toluene (bottom). 

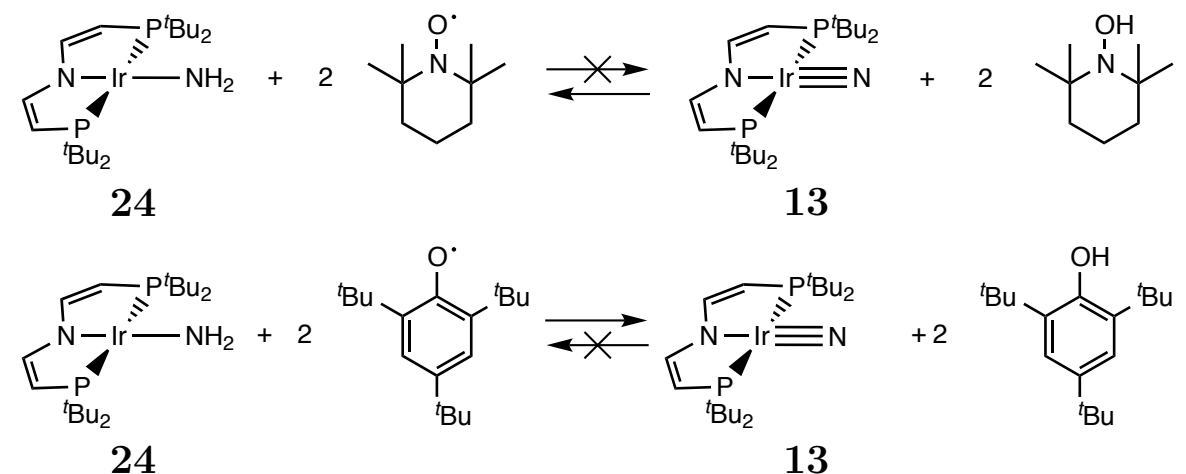

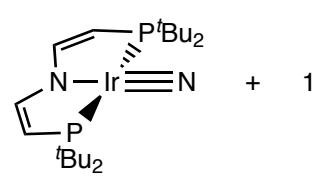

13
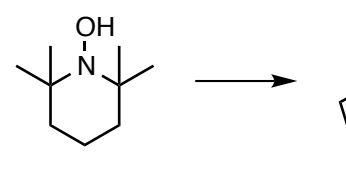

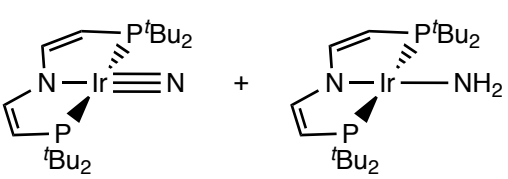

13

Scheme II.4.6: Dependency of the course of HAT reactivity of 24 and 13 on the respective reagent.

The second approach for imide synthesis followed hydrogen atom abstraction from neutral parent amido complex (scheme II.4.5). Remarkably, $\mathbf{2 4}$ is stable in solution containing excess of TEMPO radical, as followed by ${ }^{1} \mathrm{H}$ NMR spectroscopy (figure II.4.12 top). In contrast, full conversion of $\mathbf{2 4}$ under formation of $\mathbf{1 3}$ and its coupling product $\mathbf{1 4}$ is achieved upon conversion with 2,4,6-tri-tert-butylphenoxyl radical (figure II.4.12). ${ }^{6}$

\footnotetext{
${ }^{6}$ Only broad signals in the aliphatic and aromatic region of the ${ }^{1} \mathrm{H}$ NMR spectrum were assigned to 2,4,6-tri-tert-butylphenol. Note that one yet unidentified signal at a chemical shift of $\delta=4.26 \mathrm{ppm}$ was found, that was not assigned to any Ir containing complex due to the absence of any further signals within the ${ }^{31} \mathrm{P}$ NMR spectrum.
} 


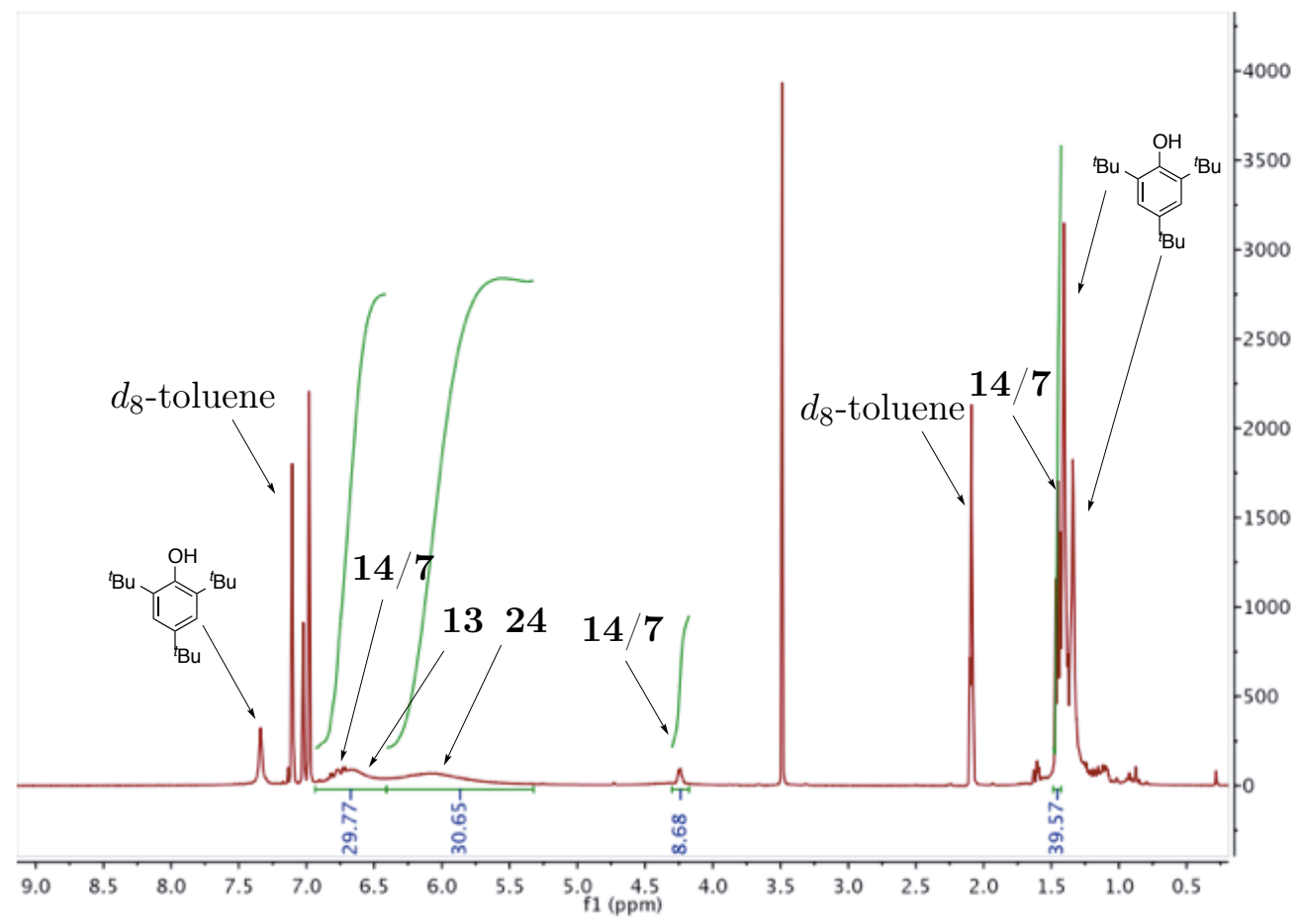

Figure II.4.11.: Room temperature ${ }^{1} H$ NMR spectrum after dissolving an equimolar mixture of amido complex 24 and 2,4,6-tri-tert-butylphenoxyl radical in $d_{8}$-toluene. 


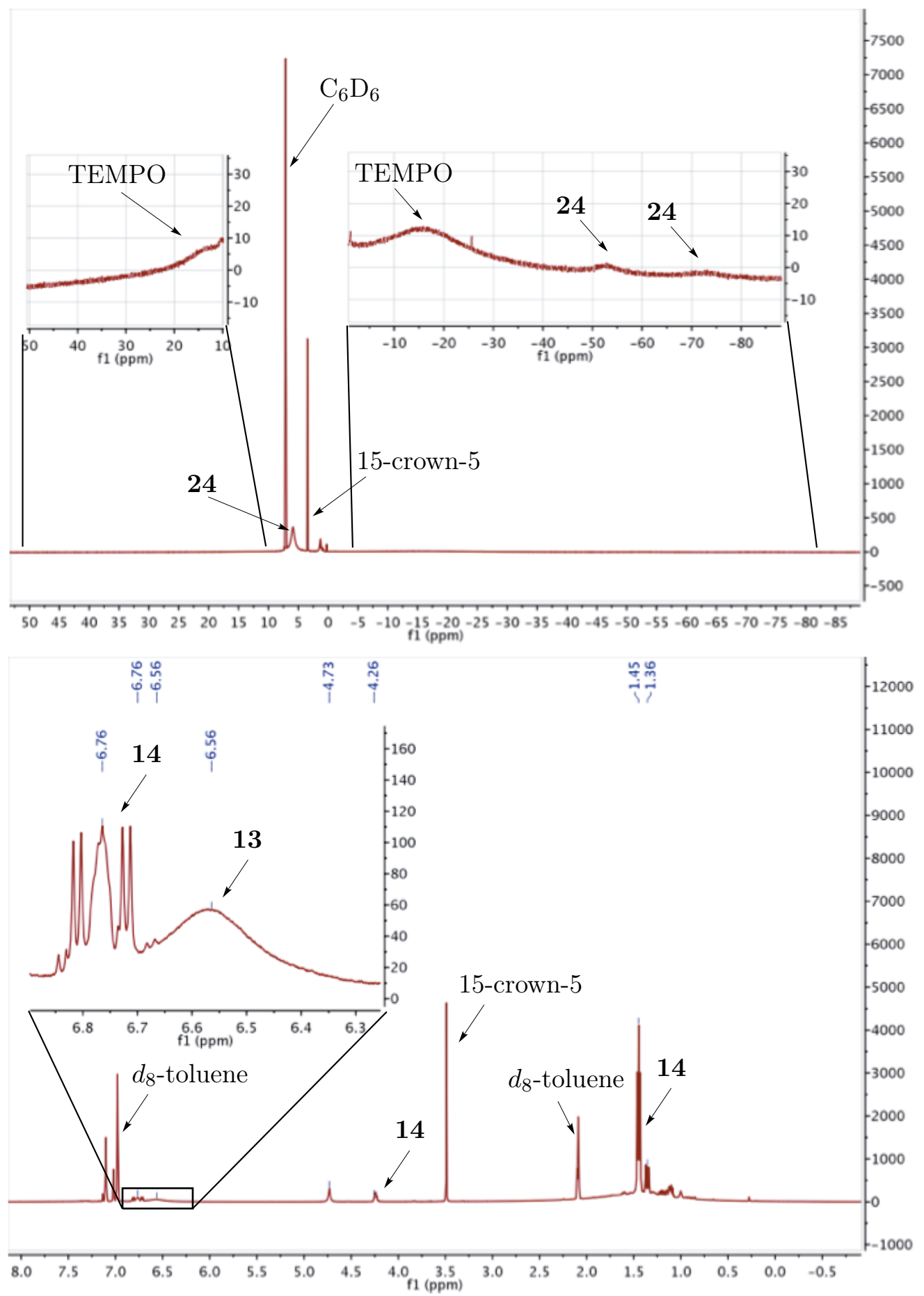

Figure II.4.12.: Top: ${ }^{1} H$ NMR spectrum of a mixture of 24 and TEMPO radical at room temperature in $C_{6} D_{6}$. Bottom: ${ }^{1} H$ NMR spectrum at room temperature of a mixture of 24 and 2,4,6-tris-tert-butylphenoxyl radical in $d_{8}$-toluene. 
The third route, namely the conversion of $\mathbf{2 1}$ in THF solution with a base such as $\mathrm{K}\left[\mathrm{N}\left(\mathrm{SiMe}_{3}\right)_{2}\right]$ or $\mathrm{KO}^{t} \mathrm{Bu}$ resulted in full conversion of the starting material indicated by the absence of its signals in the ${ }^{1} \mathrm{H}$ and ${ }^{31} \mathrm{P}$ NMR spectrum, however, did not result in neutral imido complex 22. Instead, a mixture consistent of 24 (66\%), 14 and minor amounts of $\mathbf{7}\left(33 \%\right.$ in total), and $\mathrm{H}\left[\mathrm{N}\left(\mathrm{SiMe}_{3}\right)_{2}\right](\delta=-0.03 \mathrm{ppm})$ as determined by ${ }^{1} \mathrm{H}$ NMR integration was obtained (figure II.4.13 top). Monitoring the reaction at low temperatures by NMR spectroscopy at $-60{ }^{\circ} \mathrm{C}$ revealed full conversion of $\mathbf{2 1}$ and half conversion of $\mathrm{K}\left[\mathrm{N}\left(\mathrm{SiMe}_{3}\right)_{2}\right](\delta=-0.23 \mathrm{ppm})$ in time of mixing and allows for the observation of 12 yet unidentified broad singlets with ${ }^{1} \mathrm{H}$ NMR chemical shifts of $\delta=-189.0$, $-99.2,-37.0,1.24,4.03,5.52,5.42,5.52,6.27,7.57,9.4$ and $11.2 \mathrm{ppm}$, which disappear within 5 minutes under formation of the final product mixture as obtained by reacting at room temperature (figure II.4.13 bottom). Additionally, trace amounts of neutral nitrido complex 13 were detected in times of mixing at low and room temperature.

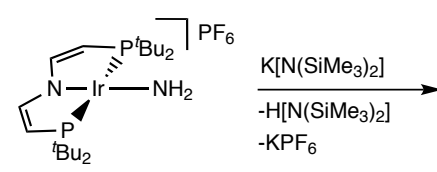

21

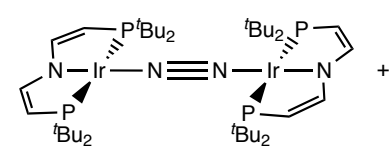

14

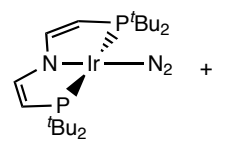

7

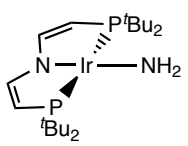

24

Scheme II.4.7: Deprotonation products of 21 resulting in 14, 7 and 24 .

Further insight into the conversion of $\mathbf{2 1}$ were provided by electrochemical investigations. The cyclic voltammogram of $\mathbf{2 4}$ in the presence of $\mathrm{K}\left[\mathrm{N}\left(\mathrm{SiMe}_{3}\right)_{2}\right]$ (10 fold excess, figure II.4.14 top) exhibits an irreversible Ir ${ }^{\mathrm{II}} / \mathrm{III}$ redox couple. Furthermore, a new redox wave at a potential of $\mathrm{E}_{1 / 2}=-0.86 \mathrm{~V}$ appears after completing the first cycle (figure II.4.14 bottom) and is attributed to the $\mathbf{1 2} / \mathbf{1 3}$ redox couple by comparison with an authentic sample of 12 in THF. Remarkably, the current drops after passing the anodic peak potential of $\mathrm{Ir}^{\mathrm{II}}$ oxidation and starts growing again until reaching the reversal potential. Quantification of the individual peak currents of the 24/21 and 13/12 redox couples, respectively, is obscured by the overlap of their redox waves. Decreasing of the excess of $\mathrm{K}\left[\mathrm{N}\left(\mathrm{SiMe}_{3}\right)_{2}\right]$ results in vanishing of the $\mathbf{1 2} / \mathbf{1 3}$ redox couple, a reversible 24/21 redox couple and decay of the peak current after passing the anodic peak potential (figure II.4.15 top). Electrochemical activity of $\mathrm{K}\left[\mathrm{N}\left(\mathrm{SiMe}_{3}\right)_{2}\right]$ is excluded by the absence of any features in the cyclic voltammogram of $\mathrm{K}\left[\mathrm{N}\left(\mathrm{SiMe}_{3}\right)_{2}\right]$ in THF solution at potentials between 0 and $-3.5 \mathrm{~V}$ (figure II.4.15 bottom). 


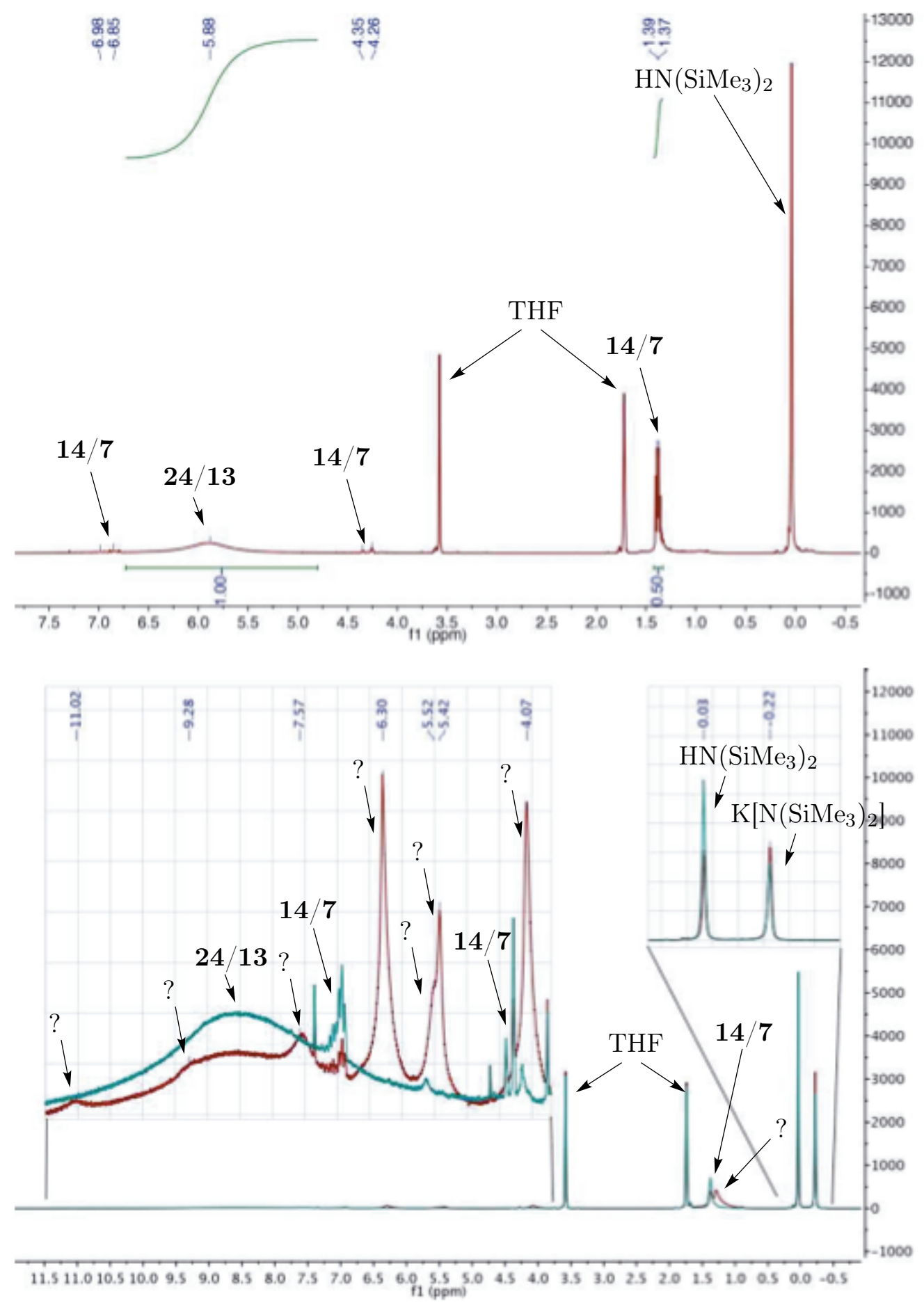

Figure II.4.13.: ${ }^{1} H$ NMR spectrum of the conversion of cationic amido complex 21 with $\mathrm{K}\left[\mathrm{N}\left(\mathrm{SiMe}_{3}\right)_{2}\right]$ in $d_{8}$-THF at room temperature (top) and superimposed ${ }^{1} \mathrm{H} N \mathrm{NM}$ spectra at $-60{ }^{\circ} \mathrm{C}$ of the same reaction directly after mixing (red curve) and after 5 minutes at room temperature (blue curve). Signals labeled with "?" could not be assigned to any Ir complex and disappear within the course of the reaction. 

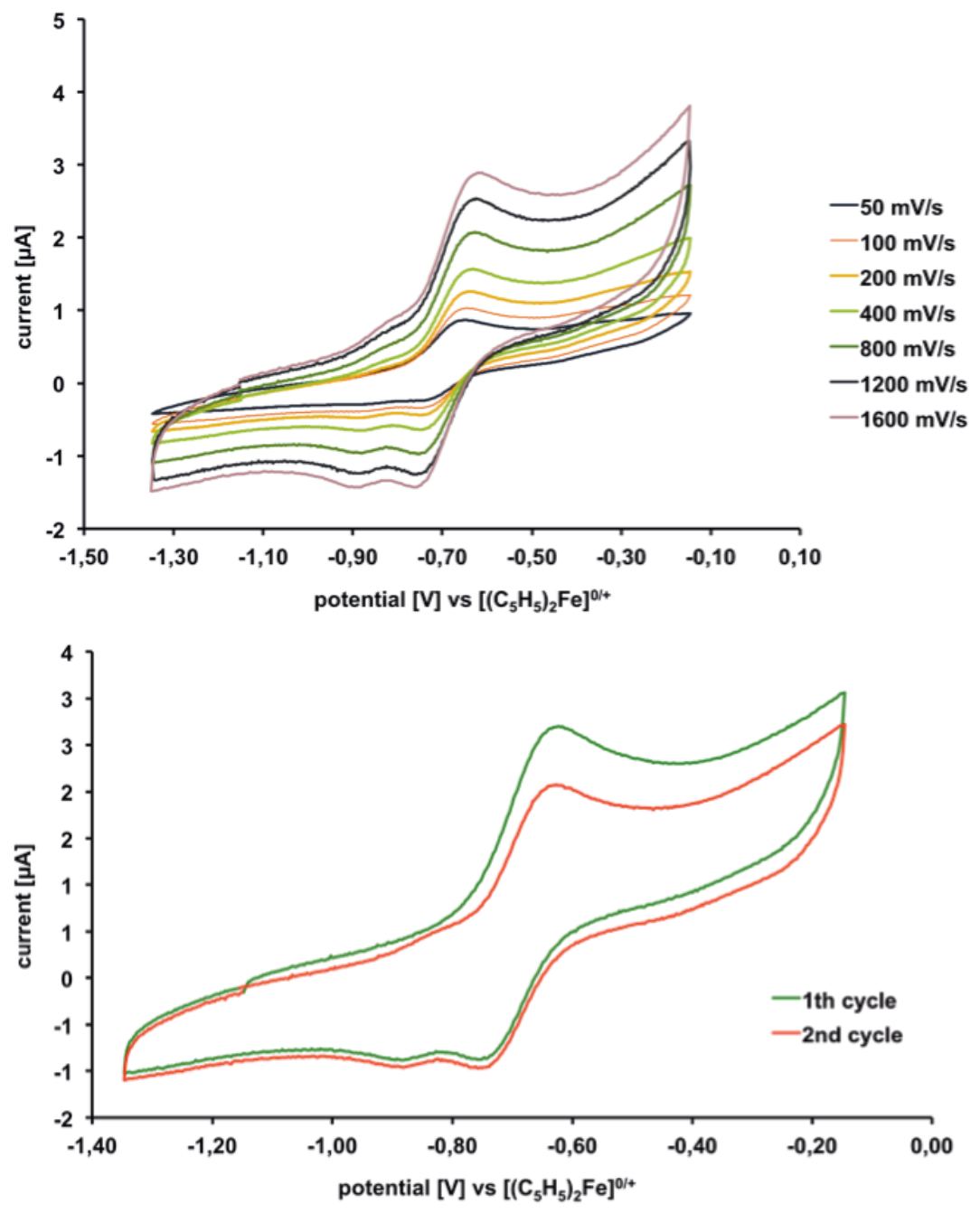

Figure II.4.14.: Cyclic voltammogram (second cycle) of $24\left(10^{-4} \mathrm{~mol} / \mathrm{L}\right)$ in THF containing $\mathrm{K}\left[\mathrm{N}\left(\mathrm{SiMe}_{3}\right)_{2}\right]\left(10^{-3} \mathrm{~mol} / \mathrm{L}\right)$ at different scan rates (top) and separation of first and second cycle at a scan rate of $800 \mathrm{mV} / \mathrm{s}$ (bottom) ([nBu $\mathrm{N}_{4} \mathrm{~N} / \mathrm{PF} \mathrm{F}_{6} 0.1 \mathrm{~mol} / \mathrm{L}, \mathrm{Pt}$ working electrode, $\mathrm{Pt}$ counter electrode, $\mathrm{Ag} / \mathrm{Ag}^{+}$reference electrode). 

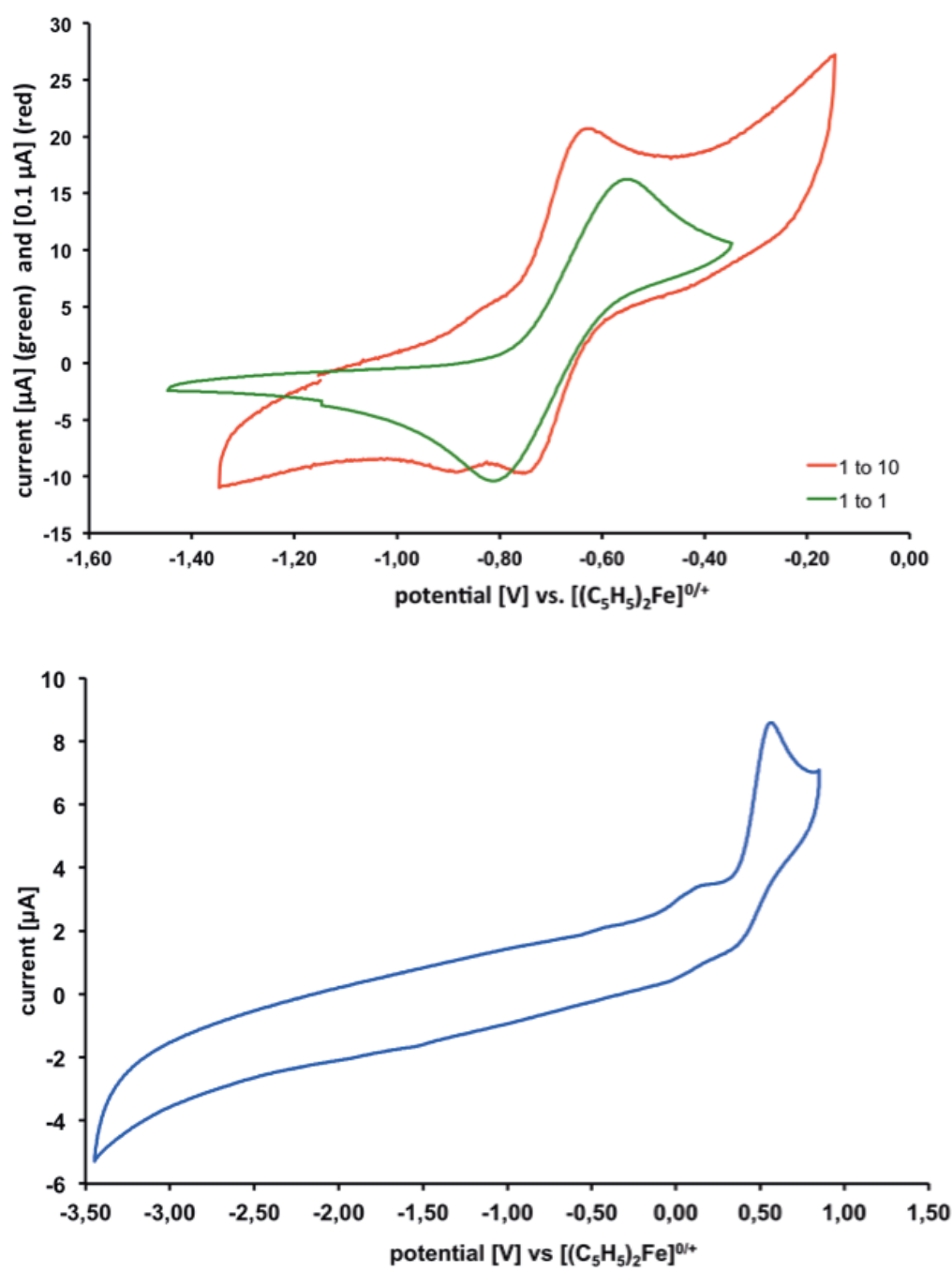

Figure II.4.15.: Top: Cyclic voltammogram of a mixture of 21 and $K\left[N\left(\mathrm{SiMe}_{3}\right)_{2}\right]$ in a 1 to 10 ratio (red line) and 1 to 1 ratio (green curve) at a scan rate of $400 \mathrm{mV} / \mathrm{s}$ in THF. The current of the red line is multiplied by 10 for better comparison. Bottom: Cyclic voltammogram of $\mathrm{K}\left[\mathrm{N}\left(\mathrm{SiMe}_{3}\right)_{2}\right]$ in $\mathrm{THF}$ at a scan rate of $400 \mathrm{mV} / \mathrm{s}$ (/nBu $u_{4} N / P F_{6} 0.1 \mathrm{~mol} / \mathrm{L}$, Pt working electrode, Pt counter electrode, $\mathrm{Ag} / \mathrm{Ag}^{+}$reference electrode). 


\subsection{Discussion}

The stability of parent amino, amido, imido and nitrido complexes of Ir can be rationalized upon considerations of the qualitative frontier molecular orbital (MO) splitting (figure II.4.16). In cationic amino complex 20, all metal electrons are localized in nonbonding, $d$-orbital based MOs. Removal of one proton under formation of neutral amido complex 24 leads to a repulsive $d \pi / p \pi$ interaction of the amides' lone pair with the occupied metal $d$-orbital, rising the energy of the $d_{x z}$ based MO and thus facilitating one electron oxidation under formation of closed-shell amido complex 21. The increasing $\pi$-donation of the amido ligand with increasing formal oxidation state of the metal is reflected within the trends of $\mathrm{Ir}-\mathrm{NH}_{2}$ distance of Ir amido complexes (vide supra).

Further deprotonation of amido complex $\mathbf{2 1}$ under formation of imido complex $\mathbf{2 2}$ entails $\pi$-interactions with a second nitrogen $p$-orbital and therefore rises the energy of a second $d$-orbital based MO. Independent of the overall spin-state of transient neutral imido complex 22, two electrons are occupied in energetically high lying MOs. Hence, it is not surprising that open-shell $\mathrm{Ir}^{\mathrm{IV}}$ nitrido complex is more stable in solution than $\mathrm{Ir}^{\mathrm{III}}$ imido complex, due to singly occupation of $\operatorname{Ir}-\mathrm{N} \pi$ anti-bonding MOs in the case of $\operatorname{Ir}^{\mathrm{IV}}$. Removal of one electron of $\mathbf{1 3}$ results in thermally stable cationic nitrido complex $\mathbf{1 2}$, with all non-bonding MOs fully occupied and empty $\pi$ anti-bonding MOs with respect to the nitride.

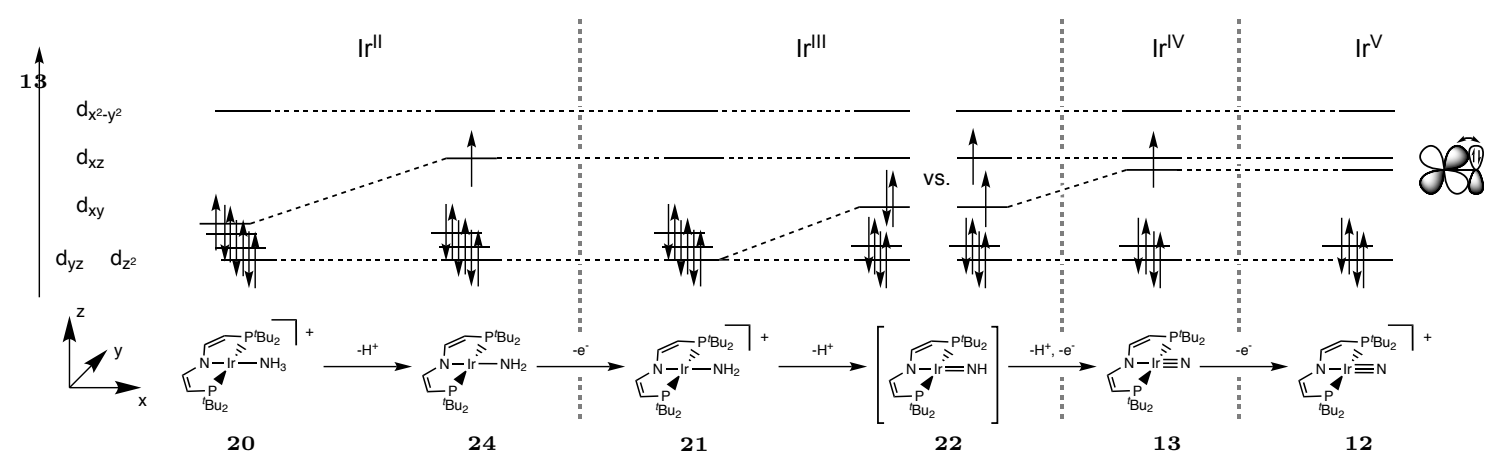

Figure II.4.16.: Qualitative frontier orbital scheme of square-planar Ir-NH $H_{x}$ complexes $(x=0-3)$. The $\pi$-donor strength of the $N H_{x}$ ligand increases with decreasing $x$, thus rising the energy of the $d_{x z}$ and subsequently the $d_{x y}$ based molecular orbital and facilitate one electron oxidation of the corresponding complexes. 
Three different attempts for the synthesis of imido complex 22, namely deprotonation of cationic amido complex 21, single HAT to neutral nitrido complex 13 and HAT from neutral amido complex $\mathbf{2 4}$ did not allow for its characterization in solution (scheme II.4.5).

\subsubsection{HAT between iridium amide and nitride}

There were no further reaction intermediates observable upon double $\mathrm{H}$ atom abstraction from amido complex $\mathbf{2 4}$ or $\mathrm{H}$ atom addition to nitrido complex $\mathbf{1 3}$ that could be assigned to neutral imido complex $\mathbf{2 2}$ as reaction intermediate and the course of the reaction was solely dependent on the respective reagent applied. The fast conversions of 13 with TEMPO-H $\left(\mathrm{BDE}_{O-H}\left(\mathrm{C}_{6} \mathrm{H}_{6}\right)=70.0 \mathrm{kcal} / \mathrm{mol}\right)^{[271]}$ and 24 with 2,4,6-tri-tertbutylphenoxyl radical $\left(\mathrm{BDE}_{O-H}\left(\mathrm{C}_{6} \mathrm{H}_{6}\right)=81.6 \mathrm{kcal} / \mathrm{mol}\right)^{[271]}$, respectively, suggests a low kinetic barrier for the interconversions of nitride and amide via HAT. Additionally, the lack of reactivity between $\mathbf{2 4}$ and TEMPO, as well as $\mathbf{1 3}$ and 2,4,6-tri-tertbutylphenol suggest a sole thermodynamic driving force for the reactions and substantiates a dependency of the course of the reaction on the BDE of the $\mathrm{O}-\mathrm{H}$ bond of the reagent. Hence, the average $\mathrm{BDE}$ over both $\mathrm{N}-\mathrm{H}$ bonds of amido complex $\mathbf{2 4}$ is assigned in between the BDE of the $\mathrm{O}-\mathrm{H}$ bond of TEMPO- $\mathrm{H}$ as lower and the $\mathrm{O}-\mathrm{H}$ bond of 2,4,6-tri-tert-butylphenol as upper limit (scheme II.4.6).

The selectivity for N-N coupling with respect to HAT of nitrido complex $\mathbf{1 3}$ in the presence of 1,4-cyclohexadiene $\left(\mathrm{BDE}_{C-H}\right.$ (gas) $\left.=76.0 \mathrm{kcal} / \mathrm{mol}^{[226]}\right)$ can be rationalized as follows: a) Double HAT of nitrido complex 13 from 1,4-cyclohexadiene is thermodynamically uphill. b) The difference between the total energy of between the products from single HAT (22 and cyclohexadienyl radical) and the starting materials (13 and 1,4-cyclohexadiene) is higher in energy than the kinetic barrier for $\mathrm{N}-\mathrm{N}$ coupling would also favor formation of $\mathrm{N}_{2}$, even if the double HAT from 1,4-cyclohexadiene to nitride $\mathbf{1 3}$ under formation of amide $\mathbf{2 4}$ and benzene is thermodynamically downhill. c) A higher kinetic barrier for the first $\mathrm{H}$ atom abstraction of 1,4-cyclohexadiene by nitride $\mathbf{1 3}$ compared with the barrier for $\mathrm{N}-\mathrm{N}$ coupling would also explain the observed selectivity for $\mathrm{N}-\mathrm{N}$ coupling.

A thermodynamic regime suggests proceeding of the reverse reaction. Hence, a) is ruled out due to the stability of amido complex 24 in benzene. In the case of b), the similar BDEs of the average $\mathrm{N}-\mathrm{H}$ bond of amide $\mathbf{2 4}$ and the $\mathrm{C}-\mathrm{H}$ bond of 1,4-cyclohexadiene would entail a very low barrier for the $\mathrm{N}-\mathrm{N}$ coupling reaction of $\mathbf{1 3}$ and is rather un- 
likely with respect to the selectivity for HAT of nitride $\mathbf{1 3}$ in the presence of TEMPO-H. Hence, a kinetic resolution c) is favored to rationalize the selectivity for $\mathrm{N}_{2}$ formation of nitride $\mathbf{1 3}$ in the presence of 1,4-cyclohexadiene, suggesting a higher kinetic barrier for HAT of nitride $\mathbf{1 3}$ with 1,4-cyclohexadiene as compared with the barrier for $\mathrm{N}-\mathrm{N}$ coupling reaction. However, a final discrimination between b) and c) is not possible, based on the experimental data.

Conversion of an equimolar mixture of nitride $\mathbf{1 3}$ and TEMPO-H results in nitride $\mathbf{1 3}$ and amide $\mathbf{2 4}$ in a 1:2 ratio, besides the dinitrogen complexes $\mathbf{1 4}$ and $\mathbf{7}$. The same product mixture was also obtained on the reverse reaction, namely the conversion of equimolar amounts of amide $\mathbf{2 4}$ and 2,4,6-tri-tert-butylphenoxyl radical.

The dinitrogen complexes $\mathbf{7}$ and $\mathbf{1 4}$ are observed as side-products in all conversions that proceed under consumption or formation of nitrido complex 13, due to its thermal lability at room temperature. Unfortunately, the ${ }^{1} \mathrm{H}$ NMR signals of amido complex $\mathbf{2 4}$ and nitrido complex 13 suitable for ${ }^{1} \mathrm{H}$ NMR integration are only separated at room temperature and overlap upon reduced measurement temperature. However, the decay of nitrido complex $\mathbf{1 3}$ as HAT starting material or product under formation of dinitrogen complexes was observed within the timescale as reported earlier. ${ }^{[200]}$ Hence, the presence of dinitrogen complexes $\mathbf{7}$ and $\mathbf{1 4}$ might be rationalized as thermodynamically independent from imide reactivity and formed subsequently due to the transient character of nitrido complex 13.

Following this assumption, the mixture of nitride and amide as disproportionation products of neutral imido complex $\mathbf{2 2}$ are rationalized in terms of a lower total energy of the reaction products $\mathbf{1 3}$ and $\mathbf{2 4}$ over two equivalents of starting $\mathbf{2 2}$ (scheme II.4.8). Hence, energy is gained upon cleavage of the first $\mathrm{N}-\mathrm{H}$ bond within imido complex $\mathbf{2 2}$ and formation of a second $\mathrm{N}-\mathrm{H}$ bond in $\mathbf{2 4}$, suggesting a higher BDE for the second $\mathrm{N}-\mathrm{H}$ bond in $\mathbf{2 4}$ over the first. Note that the assumption of independent nitride formation and subsequent $\mathrm{N}-\mathrm{N}$ coupling is not possible when cationic amido complex $\mathbf{2 1}$ is deprotonated, due to the small quantities of nitride complex $\mathbf{1 3}$ observed in time of mixing (vide infra). A lower BDE for the first $\mathrm{N}-\mathrm{H}$ bond than for the second is further substantiated by $\mathrm{C}-\mathrm{H}$ activation of toluene of the imide, however, not from the nitride. When $\operatorname{Ir}^{\mathrm{IV}}$ nitrido complex 13 is generated in toluene solution, only $\mathrm{N}-\mathrm{N}$ coupling under formation of $\mathrm{N}_{2}$ complexes $\mathbf{7}$ and $\mathbf{1 4}$ is observed. Remarkably, generation of the nitride in the presence of one equivalent of TEMPO- $\mathrm{H}$ results in a solution containing amido complex $\mathbf{2 4}$ as 


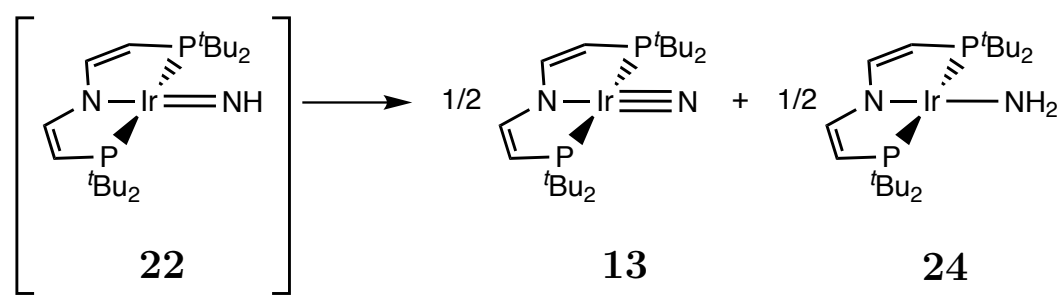

Scheme II.4.8: Disproportionation of imido complex 22 into amide 24 and nitride 13.

reaction product and $\mathrm{N}_{2}$ complexes. ${ }^{7}$ Full conversion of nitride with TEMPO-H under formation of imido complex 22 is suggested, and activation of the weak benzylic $\mathrm{C}-\mathrm{H}$ bond of toluene $\left(\mathrm{BDE}(\mathrm{gas})=89 \mathrm{kcal} / \mathrm{mol}^{[226]}\right)$ results in the generation of the amide. In contrast, the product mixture from stoichiometric conversion of amide $\mathbf{2 4}$ with 2,4,6tri-tert-butylphenoxyl radical suggest only disproportionation of transient imido complex 22. A viable explanation for this difference in degradation pathways of $\mathbf{2 2}$ might be based on a kinetic resolution of the two HAT reactions forming different $\mathrm{N}-\mathrm{H}$ bonds, as it is provided upon assuming a faster rater for HAT upon formation of the first $\mathrm{N}-\mathrm{H}$ bond in amido complex $\mathbf{2 4}$ as compared with the rate for formation of the second. Consequently, full conversion of nitrido complex 13 upon reaction with one equivalent TEMPO-H under formation of imide $\mathbf{2 2}$ is suggested and subsequent $\mathrm{C}-\mathrm{H}$ abstraction of the latter from the solvent toluene forms $\mathbf{2 4}$. In addition, $\mathrm{C}-\mathrm{H}$ abstraction under formation of the second $\mathrm{N}-\mathrm{H}$ bond is assumed to be faster than disproportionation. On the other hand, generation of imido complex $\mathbf{2 2}$ from the amide $\mathbf{2 4}$ and 2,4,6-tri-tert-butylphenoxyl radical is suggest to be slow, and the second HAT transfer is assumed to be faster. Hence, the conversion of in situ formed imide $\mathbf{2 2}$ with the phenoxyl radical is faster than its generation from the amide and the phenoxyl radical. Therefore, only the half amount of amide but all of the phenoxyl radical is converted and is in agreement with the observed product mixture consistent of $\mathbf{1 3}$, the dinitrogen complexes as side products derived from $\mathrm{N}-\mathrm{N}$ coupling and the amide $\mathbf{2 4}$ as unconverted starting material.

$$
70 \mathrm{kcal} \mathrm{mol}^{-1}<B D E(\mathrm{NH})<B D E\left(\mathrm{NH}_{2}\right)<81.6 \mathrm{kcal} \mathrm{mol}^{-1}
$$

\footnotetext{
${ }^{7}$ The $\mathrm{N}_{2}$ complexes are already present after generation of nitride $\mathbf{1 3}$ and are not assigned as side products from the conversion with TEMPO-H
} 


\subsubsection{Deprotonation of cationic iridium amide}

Upon deprotonation of cationic Ir amido complex 21, the formation of $\mathbf{1 3}$ as reaction intermediate is substantiated by observation of its characteristic ${ }^{1} \mathrm{H}$ NMR signals as well as by the appearance of a redox wave attributable to the $\mathbf{1 3} / \mathbf{1 2}$ redox couple by electrochemical investigations on $\mathbf{2 4}$ in basic solution. In contrast to the HAT reactions, the fast formation of dinitrogen complexes and the low concentrations of nitrido complex 13 observed after converting cationic amido complex $\mathbf{2 1}$ with base suggest a yet unknown mechanism for $\mathrm{N}_{2}$ formation.

Deprotonation of parent amido complex 21 with $\mathrm{K}\left[\mathrm{N}\left(\mathrm{SiMe}_{3}\right)_{2}\right]$ is indicated by the observation of $\mathrm{HN}\left(\mathrm{SiMe}_{3}\right)_{2}$, as well as by the irreversibility of the $\mathrm{Ir}^{\mathrm{II}} / \mathrm{Ir}^{\mathrm{III}}$ redox couple in basic solution, suggesting the formation of transient neutral imido complex $\mathbf{2 2}$ as first reaction intermediate. Viable pathways for imide degradations (scheme II.4.9) are HAT, proton transfer and electron transfer. HAT was already discussed as pathway for imide disproportionation in the previous section and is not assumed in this case according to the product composition. The following paragraphs will focus on separated proton and electron transfer reactions.

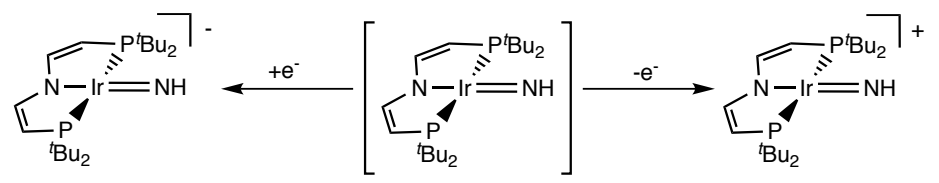

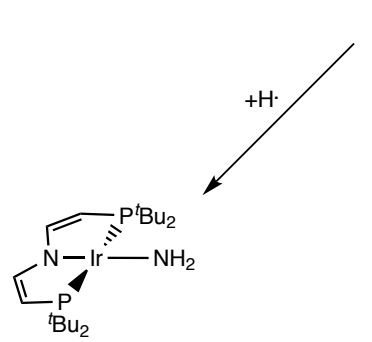

24
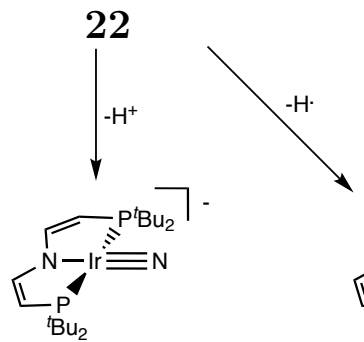

23

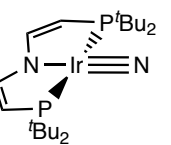

13

Scheme II.4.9: Degradation of neutral Ir ${ }^{I I I}$ imido complex 22 either via proton, electron or hydrogen atom transfer. 


\section{Proton Transfer}

Further deprotonation of neutral imido complex $\mathbf{2 2}$ would result in an anionic Ir nitrido complex. The absence of any further reversible reduction process in the cyclic voltammogram of nitrido complex 12 below the potential of the $\operatorname{Ir}^{\mathrm{V}} / \mathrm{Ir}^{\mathrm{IV}}$ redox wave questions the existence of this anionic Ir ${ }^{\mathrm{III}}$ nitrido complex. Hence, proton transfer from imido complex is not considered as initial degradation pathway of imido complex $\mathbf{2 2}$.

\section{Electron Transfer}

The electrochemical properties of transient neutral Ir imido complex $\mathbf{2 2}$ were not accessible by experiment so far, but can be estimated with respect to its electronic structure: Qualitative molecular orbital considerations of $\mathrm{Ir}^{\mathrm{III}}$ imido complex 22 suggests double occupation of Ir-N $\pi$ anti-bonding orbitals, which are singly occupied in the case of nitrido complex 13 (scheme II.4.16). Removal of one electron of these energetically high lying orbitals is expected to proceed more readily in the case of $\mathbf{2 2}$ and hence, suggesting a lower oxidation potential of $\mathbf{2 2}$ as compared with neutral nitrido complex $\mathbf{1 3}$.

This assumed low oxidation potential for $\mathrm{Ir}^{\mathrm{III}}$ imido complex 22 entails its oxidation towards cationic imido complex 23 with cationic nitride 12 (II.4.10 top), as well as with cationic amido complex 24 (II.4.10 middle). Under the conditions of both electrochemical and stoichiometric experiments, quantitative one electron oxidation of neutral imido complex $\mathbf{2 2}$ into $\mathbf{2 3}$ is expected and explains well the formation of neutral amido complex $\mathbf{2 4}$ as final reaction product and argues against reduction of the imide. Furthermore, the redox potentials of amido and nitrido complexes suggest redox reaction between neutral nitrido complex 13 and cationic amido complex 21 (scheme II.4.10 bottom).

The irreversibility of the $\mathrm{Ir}^{\mathrm{II}} / \mathrm{Ir}^{\mathrm{III}}$ redox wave of amido complexes, as well as the appearance of the redox wave assignable to nitride complexes in the cyclic voltammogram of $\mathbf{2 4}$ in basic solution suggest degradation of cationic imido complexes $\mathbf{2 3}$ proceeds at higher potentials as required for oxidation of neutral amido complex $\mathbf{2 4}$. It is rather likely that the degradation of neutral imido complex $\mathbf{2 2}$ as postulated reaction intermediate from deprotonation of cationic amido complex $\mathbf{2 1}$ proceeds mainly via initial electron transfer under formation of cationic imido complex $\mathbf{2 3}$ under electrochemical, as well as under stoichiometric investigations. 


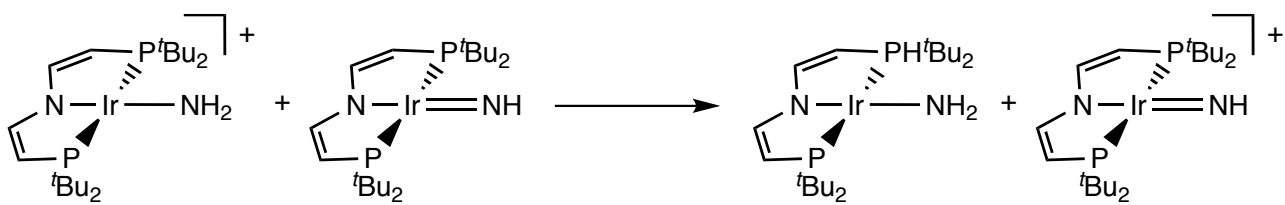

21

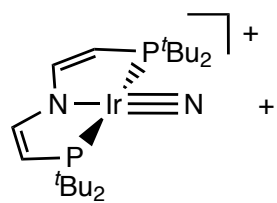

12

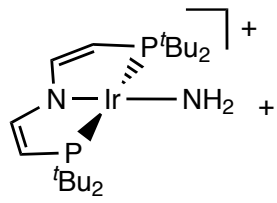

21
22

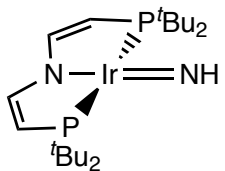

22

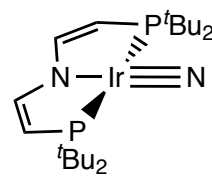

13
24

23

Scheme II.4.10: Direction of electron transfer reactions of Ir amido, imido and nitrido complexes under the assumption of a lower potential for the oxidation of neutral imido complex 22 than nitrido complex 13.

\section{Degradation of cationic imido complex}

It is tempting to assume a fast deprotonation of cationic imido complex $\mathbf{2 3}$ under formation of neutral nitrido complex $\mathbf{1 3}$ in the basic reaction mixture. The lower potential for the $\mathrm{Ir}^{\mathrm{IV} / \mathrm{V}}$ nitride redox couple as compared with the amide suggest one electron oxidation of neutral nitride with cationic amide $\mathbf{2 1}$ (scheme II.4.10 top), hence providing a viable pathway for the formation of cationic nitride 12 in both electrochemical and stoichiometric conversions. Upon electrochemical conditions, the deprotonation products of $\mathbf{2 1}$ and $\mathbf{2 3}$ are assumed to be further oxidized under formation of cationic imido and nitrido complexes. This electron transfer from the reactants in solution to the electrode is substantiated by the increase in current at potentials beyond the peak potential of the $\mathrm{III}^{\mathrm{II}} / \mathrm{Ir}^{\mathrm{III}}$ redox process.

Remarkably, the cationic nitride was never observed by ${ }^{1} \mathrm{H}$ and ${ }^{31} \mathrm{P}$ NMR spectroscopy upon stoichiometric conversion of cationic amide $\mathbf{2 1}$ with base and might provide a rationalization for the surprisingly fast rate for $\mathrm{N}_{2}$ formation. This yet unknown $\mathrm{N}-\mathrm{N}$ coupling mechanism, however, will not proceed via coupling of cationic Ir nitride $\mathbf{1 2}$ 
with neutral imido complex 22, due to their relative oxidation potentials. More likely, electron transfer between $\mathrm{Ir}^{\mathrm{V}}$ nitride and $\mathrm{Ir}^{\mathrm{III}}$ imide under formation of open-shell nitride 13 and cationic imide 23 (scheme II.4.10 middle) might initiate their bimolecular coupling reaction. Subsequent deprotonation of the first coupling intermediate forming the dinitrogen complex is expected to proceed with a different overall reaction rate than the initial deprotonation of cationic amido complex and might explain for the different rates for conversion of $\mathrm{K}\left[\mathrm{N}\left(\mathrm{SiMe}_{3}\right)_{2}\right]$. Coupling of two charged species, as it would proceed by coupling of $\operatorname{Ir}^{\mathrm{V}}$ nitride $\mathbf{1 2}$ with $\operatorname{Ir}^{\mathrm{IV}}$ imide $\mathbf{2 3}$ is rather unlikely due to repulsive coulomb interactions. Other reaction pathways for the formation of $\mathrm{N}_{2}$ complexes, such as coupling of neutral and cationic nitrido complexes and subsequent reduction of the mixed valent dinitrogen complex, however, can not be excluded.

The fast rate for degradation of nitrido complex $\mathbf{1 3}$ further hampers the differentiation between degradation pathways for the imido complex 22. Homo-coupling of the nitride for the formation of dinitrogen complex is rather slow at low temperatures and the complex reaction mixture enables different, yet only proposed mechanisms for $\mathrm{N}_{2}$ formation. Based on the observations, the following mechanism is suggested for the oxidation of neutral amido complex 24 in basic solution (scheme II.4.11): One electron oxidation of $\mathrm{Ir}^{\mathrm{II}}$ amide results in cationic amido complex 21, that is subsequently deprotonated under formation of neutral imido complex 22. The latter is oxidized either electrochemically, or by Ir III amido complex under formation of cationic imido complex 23. Upon deprotonation of $\mathbf{2 3}$, formation of neutral nitride $\mathbf{1 3}$ is initiated and is subsequently oxidized either electrochemically, or upon reaction with $\mathrm{Ir}^{\mathrm{III}}$ amido complex 21. Within this mixture of cationic and neutral $\mathrm{Ir}-\mathrm{NH}_{\mathrm{x}}$ complexes $(\mathrm{x}=0,1,2)$, irreversible coupling of neutral nitrido complex 13 with cationic imido complex $\mathbf{2 3}$ results in the formation of a protonated positively charged dimeric $\mathrm{N}_{2}$ complex that is deprotonated under the basic conditions forming dinitrogen complex 14 as final product.

Half conversion of the base upon dissolving stoichiometric mixtures of cationic amide $\mathbf{2 1}$ and $\mathrm{K}\left[\mathrm{N}\left(\mathrm{SiMe}_{3}\right)_{2}\right]$ at early reaction times can be either rationalized by two distinct deprotonation steps with different rates, or by an equilibrium in prior to the deprotonation step. However, there are a multitude of decomposition pathways for cationic amido complex 21 in the presence of the expected mixture of cationic and neutral $\mathrm{Ir}-\mathrm{NH}_{\mathrm{x}}$ complexes $(\mathrm{x}=0-2)$ in basic solution, that might contribute to the overall reaction mechanism. A final assignment to one main degradation pathway can not be performed based on these experiments. 


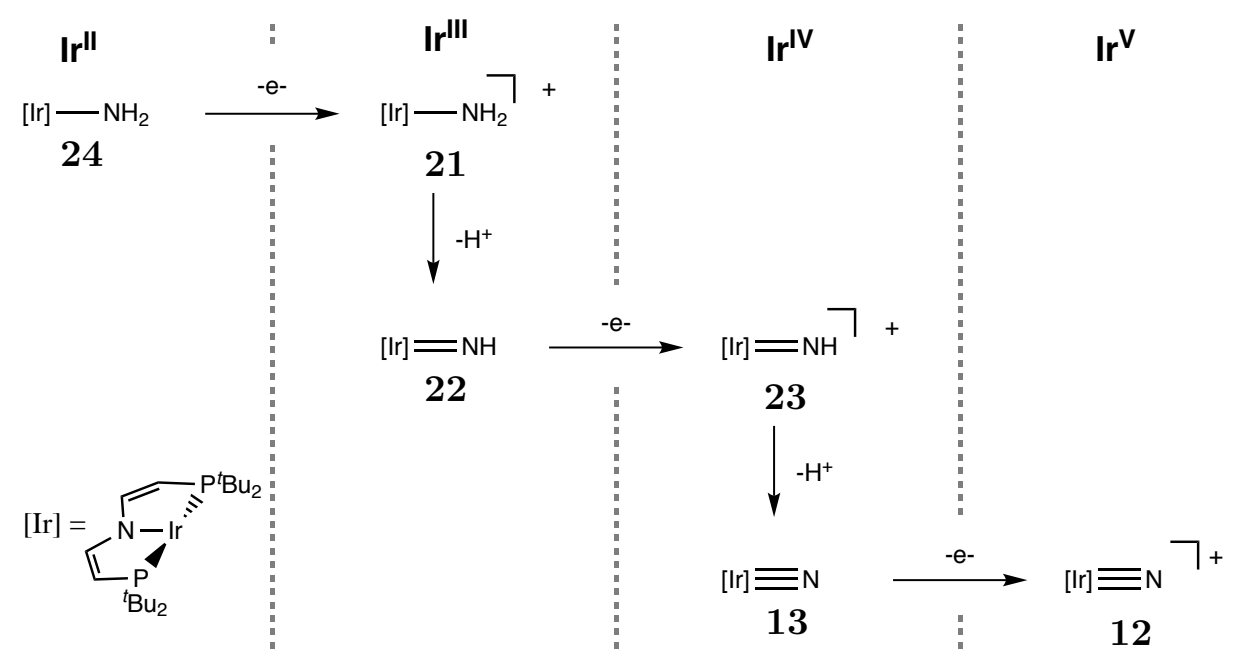

Scheme II.4.11: Proposed mechanism for the deprotonation of cationic amido complex 21, either generated electrochemically or employed as starting material. Oxidation steps are intended by reduction of cationic amide under formation of 24 as final reaction product.

\subsection{Nitride coupling versus $\mathrm{H}$-atom abstraction}

Synthesis of parent amido complex 24 and its interconversions with nitride 13 upon reaction with reagents varying in their $\mathrm{E}-\mathrm{H} \mathrm{BDE}(\mathrm{E}=$ element) enables the prediction of reaction pathways. The $\mathrm{N}-\mathrm{H}$ bond resulting from the first $\mathrm{H}$ atom abstraction reaction of nitrido complex $\mathbf{1 3}$ is weaker comparing to the second $\mathrm{N}-\mathrm{H}$ of amido complex $\mathbf{2 4}$ and explains well the transient character of imido complex 22. Disproportionation of the latter is attributed to the higher thermodynamic stability of nitride and amide, which is rationalized in the gain in energy from breaking the imide's $\mathrm{N}-\mathrm{H}$ bond under formation of the amide's $\mathrm{N}-\mathrm{H}$ bond.

The insertion of the nitride into a $\mathrm{C}-\mathrm{H}$ bond as decomposition pathway is in competition with $\mathrm{N}-\mathrm{N}$ coupling reaction. To favor the nitride insertion, the kinetic barrier as well as the total energies of the first intermediates from HAT must be lower in energy than the barrier for $\mathrm{N}-\mathrm{N}$ coupling. The formation of a second $\mathrm{N}-\mathrm{H}$ bond is energetically downhill and in agreement with the multitude of applications of imido complexes for nitrene insertions and the few examples of $\mathrm{C}-\mathrm{H}$ activation reactions performed by terminal nitrides. 


\section{Chalcogenonitrosyl complexes of iridium}

The reactivity of electron rich late terminal transition metal nitrido complexes is only sparingly explored due to the low number of isolated examples. ${ }^{[49]}$ For closed-shell Ir nitrido complex 12, electrophilic reactivity was demonstrated by formation of nitrosyl complex 25 via conversion with trimethylamine $N$-oxide. ${ }^{[200]}$ There is only one report about a full series of structurally characterized chalcogenonitrosyl complexes $\left[\mathrm{Os}(\mathrm{Tp})(\mathrm{NE}) \mathrm{Cl}_{2}\right]$ $\left(\mathrm{Tp}^{-}=\right.$tripyrazolylborate, $\mathrm{E}=$ none, $\left.\mathrm{O}, \mathrm{S}, \mathrm{Se}\right)$, which were obtained by conversion of electrophilic Os ${ }^{\mathrm{VI}}$ nitride with trimethylamine $N$-oxide, elemental sulfur or selenium. ${ }^{[189]}$ The isolobal relationship between six coordinate $\mathrm{Os}^{\mathrm{VI}}$ and square-planar $\operatorname{Ir}^{\mathrm{V}}$ nitrides suggests similar reactivities for both systems. This relationship enables the synthesis of a new series of structurally characterized chalcogenonitrosyl complexes of Ir. A bonding model of the NE moiety $(\mathrm{E}=\mathrm{O}, \mathrm{S}, \mathrm{Se})$ will be derived from the structural and spectroscopic features.

The results of this chapter are published in 2013 under the title: „Thionitrosyl- and Selenonitrosyliridium Complexes“. ${ }^{[222]}$

\subsection{Synthesis}

The electrophilic character of the Iridium nitrido moiety can be demonstrated by the conversion of $\mathbf{1 2}$ with trimethylamine $N$-oxide forming iridium PNP nitrosyl complex $\mathbf{2 5}$ (scheme II.5.1). This reactivity is typically observed for electrophilic nitrides, ${ }^{[48]}$ such as iridium nitride $\mathbf{I}^{\left[{ }^{28]}\right.}$

When an equimolar mixture of $\mathbf{1 2}$ and trimethylamine $N$-oxide is dissolved in THF or DCM, an immediate change in color from deep blue to deep purple is observable in combination with full conversion of $\mathbf{1 2}$ to solely one new diamagnetic product $\left({ }^{31} \mathrm{P}\right.$ NMR spectroscopy). Organic byproducts can be removed after crystallization out of 


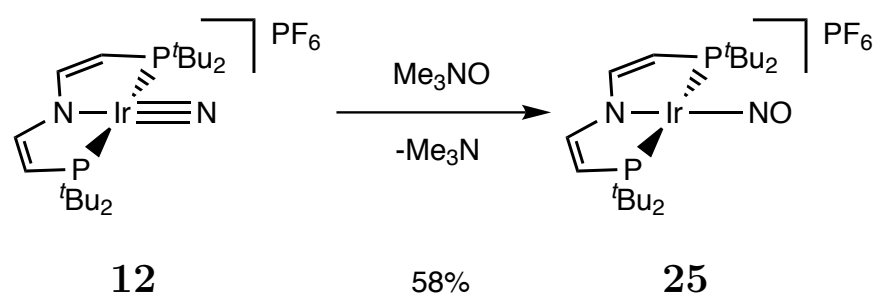

Scheme II.5.1: Oxidation of 12 with trimethylamine N-oxide under formation of Iridium PNP nitrosyl complex 25.

pentanes/DCM and $\mathbf{2 5}$ can be obtained analytically pure in $58 \%$ isolated yield.

Stirring a suspension of $\mathbf{1 2}$ and elemental sulfur at room temperature in DCM results in the slow, yet selective conversion of $\mathbf{1 2}$ into thionitrosyl complex 26, as monitored by ${ }^{31} \mathrm{P}$ NMR spectroscopy (scheme II.5.2). Analytically pure, dark-teal $\mathbf{2 6}$ was isolated in

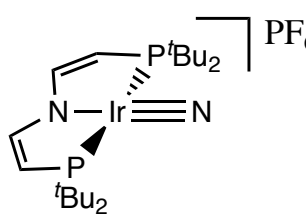

12

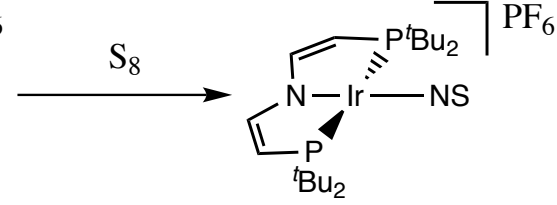

$53 \%$
26

Scheme II.5.2: Conversion nitride 12 with elemental sulfur into thionitrosyl complex 26 .

over $50 \%$ yield upon crystallization. In analogy, the reaction of $\mathbf{1 2}$ with gray selenium at $40{ }^{\circ} \mathrm{C}$ gives selenonitrosyl complex $\mathbf{2 7}$ as the main product (scheme II.5.3). In this case, unidentified side products were also observed by ${ }^{31} \mathrm{P}$ NMR spectroscopy. These products can be easily removed by crystallization, resulting in the isolation of dark-blue $\mathbf{2 7}$ in around $30 \%$ yield.

${ }^{1} \mathrm{H},{ }^{13} \mathrm{C}$, and ${ }^{31} \mathrm{P}$ NMR spectra of $\mathbf{2 5 ,} \mathbf{2 6}$ and $\mathbf{2 7}$ are in agreement with $C_{2 V}$ symmetry of the diamagnetic compounds on the NMR timescale. The almost superimposable NMR spectra point towards strongly related electronic structures for the thio- and selenonitrosyl complexes. The ${ }^{15} \mathrm{~N}$ NMR chemical shift of the thionitrosyl group ( $\delta=5.3 \mathrm{ppm} v s$. $\mathrm{Me}^{15} \mathrm{NO}_{2}$ ) obtained with an isotopically enriched sample is close to the reported value $(\delta=26.0 \mathrm{ppm})$ for $\left[\mathrm{Os}(\mathrm{Tp})(\mathrm{NS}) \mathrm{Cl}_{2}\right] .{ }^{[189]}$ 


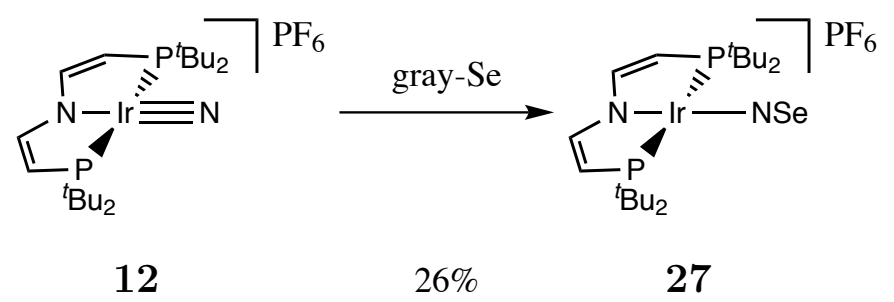

Scheme II.5.3: Conversion nitride 12 with gray selenium into selenonitrosyl complex 26 .

\subsection{IR spectroscopic characterization}

One intense band in the IR spectrum (figure II.5.1) at $1812 \mathrm{~cm}^{-1}$ is assigned to the NO stretching vibration and is similar in energy than found for nitrosyl complex derived from iridium nitride $\mathbf{I}\left(1.759 \mathrm{~cm}^{-1}\right)^{[28]}$. This observation is attributed to weaker $\mathrm{Ir} \rightarrow \mathrm{N} \equiv \mathrm{O}$ back bonding in $\mathbf{2 5}$ due to the positive charge of the complex.

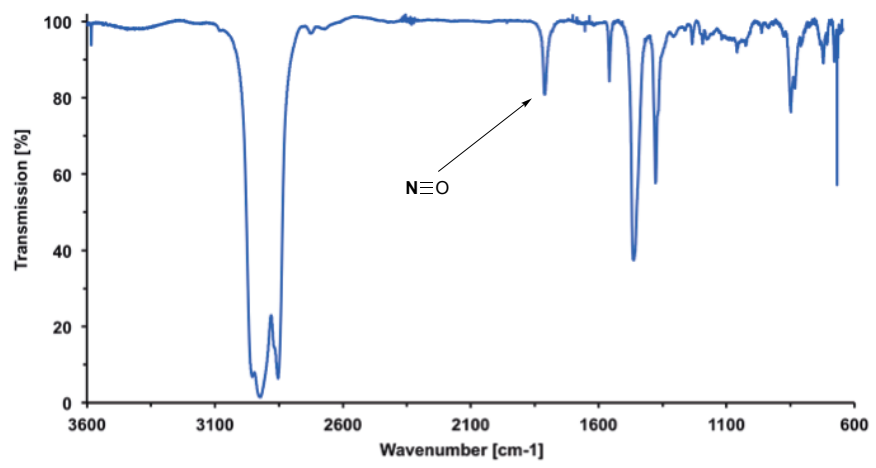

Figure II.5.1.: IR spectrum of 25 as nujol mull.

An IR peak at $1285 \mathrm{~cm}^{-1}$ (table II.5.1) could be assigned to the NS stretching vibration by ${ }^{15} \mathrm{NS}$ labeling $\left({ }^{14 / 15} \mathrm{~B}\right.$ isotopic shift: $\Delta \nu=36 \mathrm{~cm}^{-1}$, figure II.5.2). This value is in the typical range found for other thionitrosyl complexes. ${ }^{[272-274]}$ The ratio of the $\mathrm{NO} / \mathrm{NS}$ stretching frequencies (1.41) is in agreement with other reported examples $(1.40-1.41) .{ }^{[273]}$ As for these compounds, this value is considerably higher than the difference in reduced mass for NO and NS within the harmonic oscillator approximation (1.14), which is indicative of weaker N-S bonding. The N-Se stretching vibration was assigned to a peak at $1134 \mathrm{~cm}^{-1}$ by comparison with the spectra of 12, 25, 26 and $\mathbf{2 7}$. This compares well with the other experimental value $\left(1156 \mathrm{~cm}^{-1}\right)$ that was reported for 


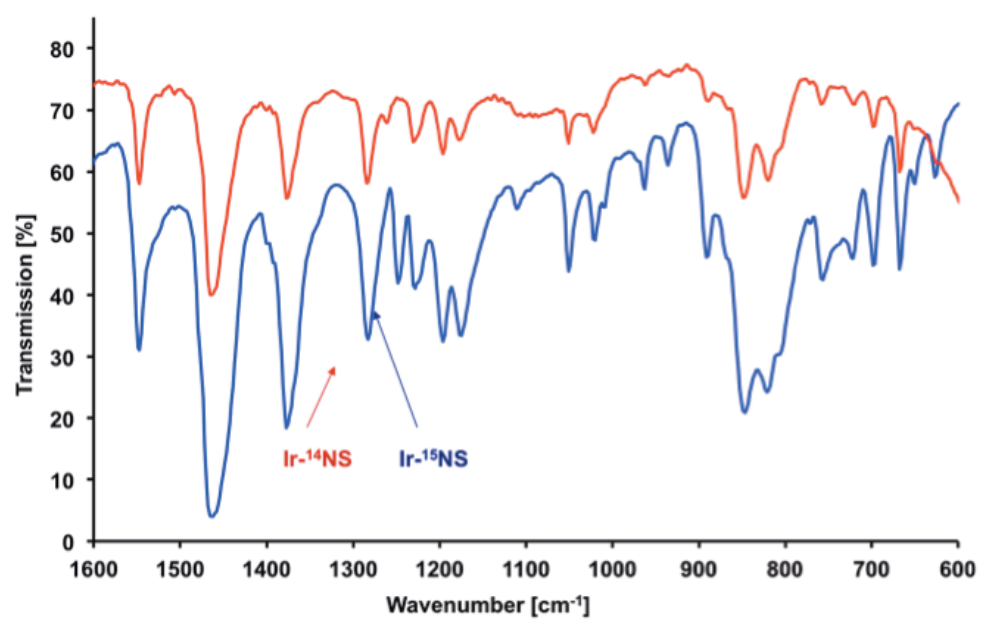

Figure II.5.2.: IR spectra (nujol mull) of 26 (red) and ${ }^{15 / 14} \mathrm{~N}-26$ (blue).

$\left[\mathrm{Os}(\mathrm{Tp})(\mathrm{NSe}) \mathrm{Cl}_{2}\right] \cdot{ }^{[189]}$ As for this Os-NE series, the ratio of stretching frequencies of the thionitrosyl (26) versus selenonitrosyl (27) complexes (1.13) is close to the harmonic oscillator approximation (1.11), which indicates similar N-E bond strengths within the heavier chalcogenonitrosyl ligands.

\subsection{Discussion of the molecular structures}

Suitable single crystals of $\mathbf{2 5}, \mathbf{2 6}$ and $\mathbf{2 7}$ were obtained to determine the molecular structures by single crystal X-ray diffraction, representing the second example of a crystallogrphically characterized chalcogenonitrosyl $\mathrm{M}-\mathrm{NE}\left(\mathrm{E}=\mathrm{O}, \mathrm{S}, \mathrm{Se}\right.$, none ${ }^{[200]}$ figure II.5.3) series. ${ }^{1}$ Compounds $\mathbf{2 5}$ and $\mathbf{2 6}$ crystallize in the monoclinic $P 2_{1} / m$ space group and exhibit isostructural behavior, whereas selenium-substituted compound $\mathbf{2 7}$ crystallizes in the monoclinic $C 2 / c$ space group. All three structures confirm the square-planar coordination geometry around the Ir atom. The Ir(PNP) fragments exhibit similar structural features, as expressed, for example, in the Ir- $\mathrm{N}_{\mathrm{PNP}}$ bond lengths (25: 1.991(2) $\AA$, 26: $1.995(2) \AA, 27: 1.997(3) \AA)$. These values are in the same range as that found 

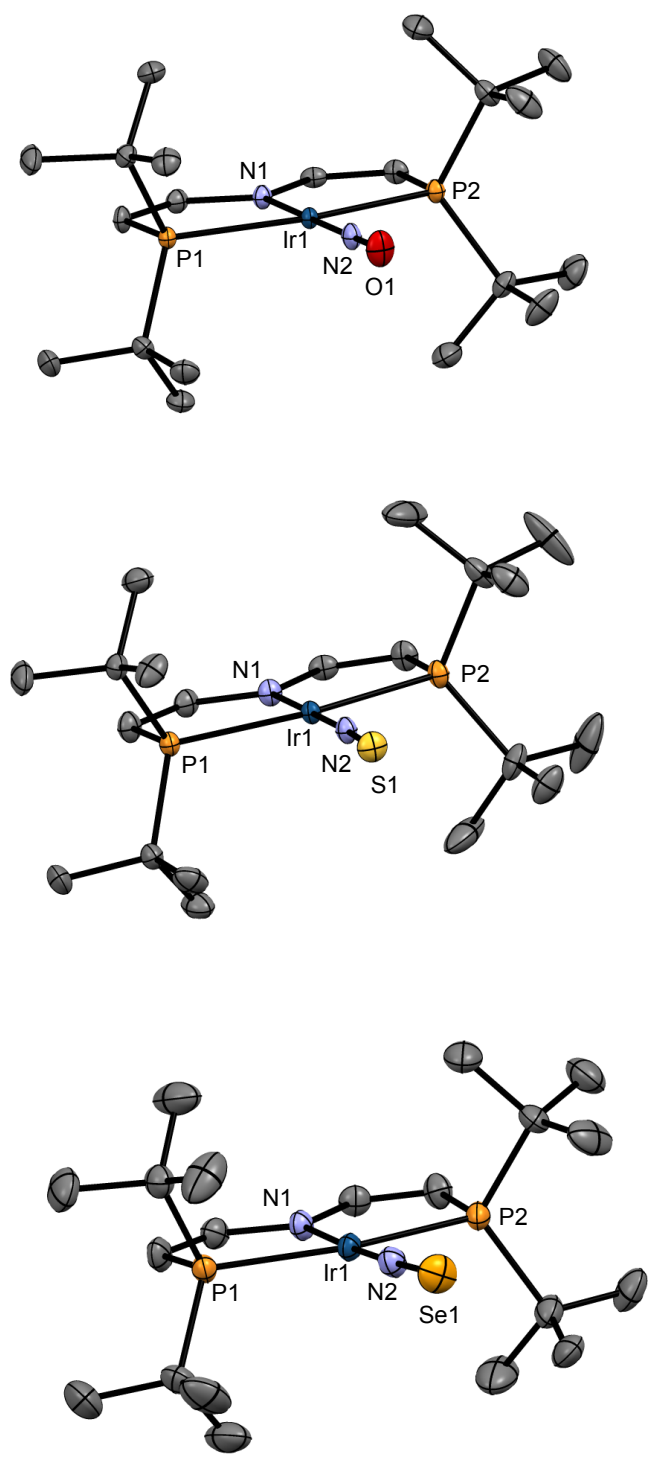

Figure II.5.3.: Molecular structures of 25, 26 and 27 (H atoms and $P F_{6}$ counter ions are omitted for clarity). The anisotropic displacement parameters are depicted at 50\% probability level. Selected bound lengths [A] and angles [\%: 25: Ir1-N1 1.991(2), Ir1-N2 1.749(2), N2-O1 1.168(3), Ir1-P1 2.3650(11), Ir1-P2 2.3640(12); N2-Ir1-N1 179.70(10), P1-Ir1-P2 163.43(3), O1-N2-Ir1 179.5(2). 26: Ir1-N1 1.995(2), Ir1-N2 1.768(2), N2-S1 1.1588(2), Ir1-P1 2.3552(7), Ir1-P2 2.3558(7); N2-Ir1-N1 179.42(8), P1-Ir1-P2 163.35(2), S1-N2-Ir1 176.97(14). 27: Ir1-N1 1.997(3), Ir1-N2 1.756(4), N2-Se1 1.678(4), Ir1-P1 2.3580(12), Ir1-P2 2.3614(12); N2-Ir1-N1 177.80(19), P1-Ir1-P2 162.99(4), Se1-N2-Ir1 177.4(2). 
in $[\operatorname{Ir}(\mathrm{PNP}) \mathrm{Cl}](1.985(2) \AA),{ }^{[163]}$ yet they are shorter relative to found in parent 12 $(2.041(4) \AA)^{[200]}$ as a consequence of the stronger nitride trans influence.

All three Ir-N-E moieties are almost linear. Interestingly, the NSe ligand of the only other selenonitrosyl complex (i.e., $\left[\mathrm{Os}(\mathrm{Tp})(\mathrm{NSe}) \mathrm{Cl}_{2}\right]$ ) is considerably more bent $\left(164.7(6)^{\circ}\right) .{ }^{[189]}$ The Ir-NE bonds (table II.5.1) are only slightly longer (0.07-0.09 $\AA$ ) relative to those of parent $12(1.678(4) \AA)$, which possibly indicates multiple-bond character with the metal atom. This elongation compares well with the Os series $\left[\mathrm{Os}(\mathrm{Tp}) \mathrm{Cl}_{2}(\mathrm{NE})\right](\mathrm{E}=$ none: 1.70(2) $\AA$; S: $1.800(8) \AA$, Se; $1.800(10) \AA)$, whereas [Os(Tp) $\left.\mathrm{Cl}_{2}(\mathrm{NO})\right]$ exhibits a slightly shorter Os-N bond (1.65(3) $\AA$ ) than the parent nitride. The N-E bond length in $\mathbf{2 5 , 2 6}$ and $\mathbf{2 7}$ is in the typical range for $\mathrm{N}-\mathrm{E}$ double bonds. ${ }^{[275]}$

\subsection{Discussion of the bonding model}

The spectroscopic and structural features of the full 12, 25, 26 and 27 series reveal interesting trends in the context of bonding models discussed for $\mathrm{M}-\mathrm{N}$ multiple bonding. The linearity of the Ir-N-E moieties are expected of $\{\mathrm{M}(\mathrm{NE})\}^{4}$ complexes within the description of the Enemark-Feltham notation. ${ }^{[276]}$ This notation avoids ambiguities in the assignment of the metal oxidation state to account for extensive $\pi$-electron delocalization. These considerations generally apply for parent nitrido complexes as well. ${ }^{[277]}$

Table II.5.1.: Selected infrared and crystallographic data for $[\operatorname{Ir}(N E)(P N P)] P F_{6}$ compounds 12, 25, 26 and 27.

\begin{tabular}{cccccc}
\hline Compound & $\mathrm{E}$ & $\begin{array}{c}\nu(\mathrm{N}-\mathrm{E}) \\
{\left[\mathrm{cm}^{-1}\right]}\end{array}$ & $\begin{array}{c}d(\mathrm{~N}-\mathrm{E}) \\
{[\AA]}\end{array}$ & $\begin{array}{c}d(\mathrm{Ir}-\mathrm{NE}) \\
{[\AA]}\end{array}$ & $\begin{array}{c}(\mathrm{Ir}-\mathrm{N}-\mathrm{E}) \\
{\left[{ }^{\circ}\right]}\end{array}$ \\
\hline $\mathbf{1 2}$ & - & - & - & $1.678(4)^{[200]}$ & - \\
$\mathbf{2 5}$ & $\mathrm{O}$ & 1812 & $1.168(3)$ & $1.749(2)$ & $179.5(2)$ \\
$\mathbf{2 6}$ & $\mathrm{S}$ & 1285 & $1.522(29$ & $1.768(2)$ & $176.97(14)$ \\
$\mathbf{2 7}$ & $\mathrm{Se}$ & 1134 & $1.678(4)$ & $1.756(4)$ & $177.4(2)$ \\
\hline
\end{tabular}

The high degree of covalency within Ir-N $\pi$ bonding of $\mathbf{1 2}$ and $\mathbf{1 3}$ was demonstrated, and a spectroscopic oxidation state closer to $\mathrm{Ir}^{\mathrm{III}}$ rather than formal $\mathrm{Ir}^{\mathrm{V}}$ was proposed for $\mathbf{1 2}$

\footnotetext{
${ }^{1}$ X-ray diffraction was performed in collaboration with Prof. Dr. Dietmar Stalke, Dipl.-Chem. Hilke Wolf and M. Sc. Peter Stollberg.
} 
(vide supra). ${ }^{[200]}$ Gray et al. recently pointed out that the electronic ground-state configuration of $d^{2}$ ions in octahedral nitride complexes $\left(\left[\mathrm{M}(\mathrm{N}) \mathrm{L}_{5}\right]^{n}\right)$, that is, $(x y)^{2}(x z, y z)^{0}\left(x^{2}\right.$ $\left.y^{2}\right)^{0}\left(z^{2}\right)^{0},{ }^{[54,184,278]}$ also strongly contributes to the corresponding nitrosyl $\left[\mathrm{M}(\mathrm{NO}) \mathrm{L}_{5}\right]^{n}$ electronic structure. In contrast, the $(x y)^{2}(x z, y z)^{4}\left(x^{2}-y^{2}\right)^{0}\left(z^{2}\right)^{0}$ resonance description overemphasizes the metal redox state change upon oxygen transfer. ${ }^{[279,280]}$ It is tempting to apply this description to our isolobal, square-planar Ir series, and the close metric parameters within $\mathbf{1 2}$ and $\mathbf{2 5}, \mathbf{2 6}$ and $\mathbf{2 7}$ strengthen that view. For the calculated series $\left[\mathrm{Re}(\mathrm{NE}) \mathrm{Cl}_{2}\left(\mathrm{PH}_{3}\right)_{3}\right](\mathrm{E}=$ none, $\mathrm{O}, \mathrm{S}, \mathrm{Se}, \mathrm{Te})$, Vyboishchikov and Frenking distinguished between $\mathrm{M}-\mathrm{NO}$ and $\mathrm{M}-\mathrm{NE}(\mathrm{E}=\mathrm{S}, \mathrm{Se}, \mathrm{Te})$ bonding. ${ }^{[281]}$ The nitrosyl complex was best described with covalent $\mathrm{Re}=\mathrm{N}=\mathrm{O}$ double bonds. In contrast, bonding analysis for the heavier chalcogens suggested a donor-acceptor interaction between the TM nitride moiety and an E atom in the singlet state as an appropriate description (figure II.5.4). In support of this model, the results from vibrational spectroscopy and slightly longer Ir$\mathrm{NO}$ versus Ir-NS/Se distances suggest stronger bonding interactions within NE for $\mathrm{E}=$ $\mathrm{O}$ than for $\mathrm{E}=\mathrm{S}$, Se.
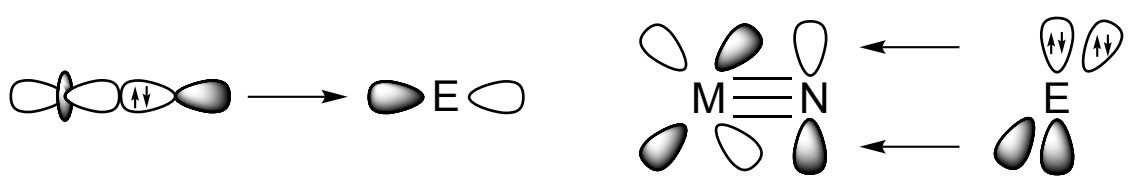

Figure II.5.4.: Description of $M N-E(E=S$, Se) bonding as a donor-acceptor interaction. [281] 



\section{Part III.}

\section{Conclusion}


Starting point of this work was the isolation and characterization of open-shell squareplanar $\mathrm{Ir}^{\mathrm{II}}$ chloro complex 3 with a $d^{7}$ electron count stabilized by the new fully dehydrogenated PNP pincer ligand (= PNP'). Based on electrochemical investigations on 3, the synthetic oxidation towards cationic 14 valence electron complex 4 in a low-spin $d^{6}$ electronic configuration was accomplished (chart III.5.1) and 4 was fully characterized. Compared to the ethylene-bridged PNP amido ligand, the PNP' pincer ligand exhibits higher conformational rigidity, but electronic flexibility: While the radical complexes from oxidation of $\mathrm{Ir}^{\mathrm{I}}$ dialkylamides are generally transient species, the metallo radical $\mathbf{3}$ and the oxidation product $\mathbf{4}$ are sufficiently stable to be easily isolated. ${ }^{[165,166,176,204]}$ Thus the new diene-amido ligand enables for examinations of an unusual one-electron reactivity of iridium.

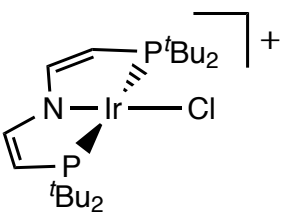

4

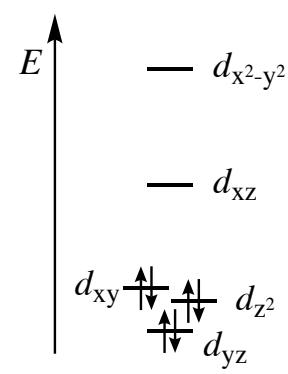

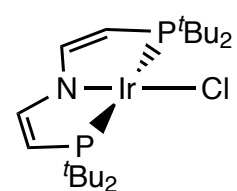

3

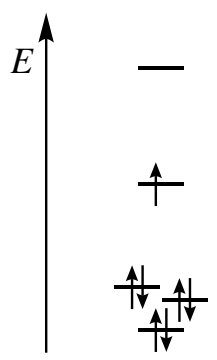

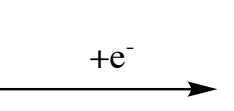

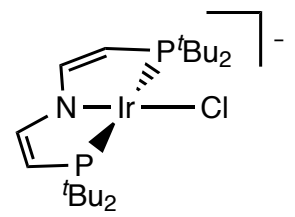

5

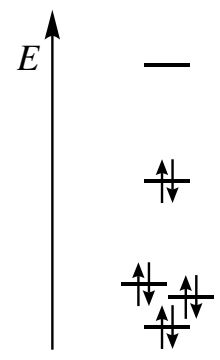

Chart III.5.1: Square-planar Ir ${ }^{I-I I I}$ PNP pincer redox series and respective qualitative metal-centered frontier orbitals based on DFT.

Additionally, isolation and characterization of the anionic $\operatorname{Ir}^{\mathrm{I}}$ chloro pincer complex $\mathbf{5}$ provides an excellent starting material for the synthesis of several $\operatorname{Ir}^{\mathrm{I}}$ and $\operatorname{Ir}^{\mathrm{III}}$ complexes by ligand substitution and oxidative addition. In contrast to $\left[\operatorname{Ir}\left(\mathrm{PMe}_{3}\right)\left(\mathrm{N}\left\{\mathrm{CH}_{2} \mathrm{CH}_{2}\right.\right.\right.$ $\left.\left.\left.\mathrm{P}^{i} \mathrm{Pr}_{2}\right\}_{2}\right)\right]^{[204]}$, exclusively metal-directed oxidative addition of MeTOf is observed, demonstrating the rigid and chemically inert character of the PNP' pincer platform. The use of chloride as a leaving group represents a new route to generate the transient $\mathrm{M}(\mathrm{PEP})$ $\left(\mathrm{M}=d^{8}\right.$ ion, $\left.\mathrm{E}=\mathrm{C}, \mathrm{N}\right)$ intermediates, which readily oxidatively add hydrocarbons, like 
benzene. In reverse, the stabilization of the $\mathrm{M}(\mathrm{PEP})$ platform by chloride emphasizes that excess halide salt formation in catalytic transformations could have a detrimental effect on $\mathrm{C}-\mathrm{H}$ activation with such pincer species, as was observed for $\mathrm{N}_{2}$. In addition, the first fully spectroscopically and structurally characterized iridium pincer $\eta^{2}-\mathrm{O}_{2}$ complex 10 was obtained, indicating the formation of an $\operatorname{Ir}^{\mathrm{III}}$ peroxo compound.

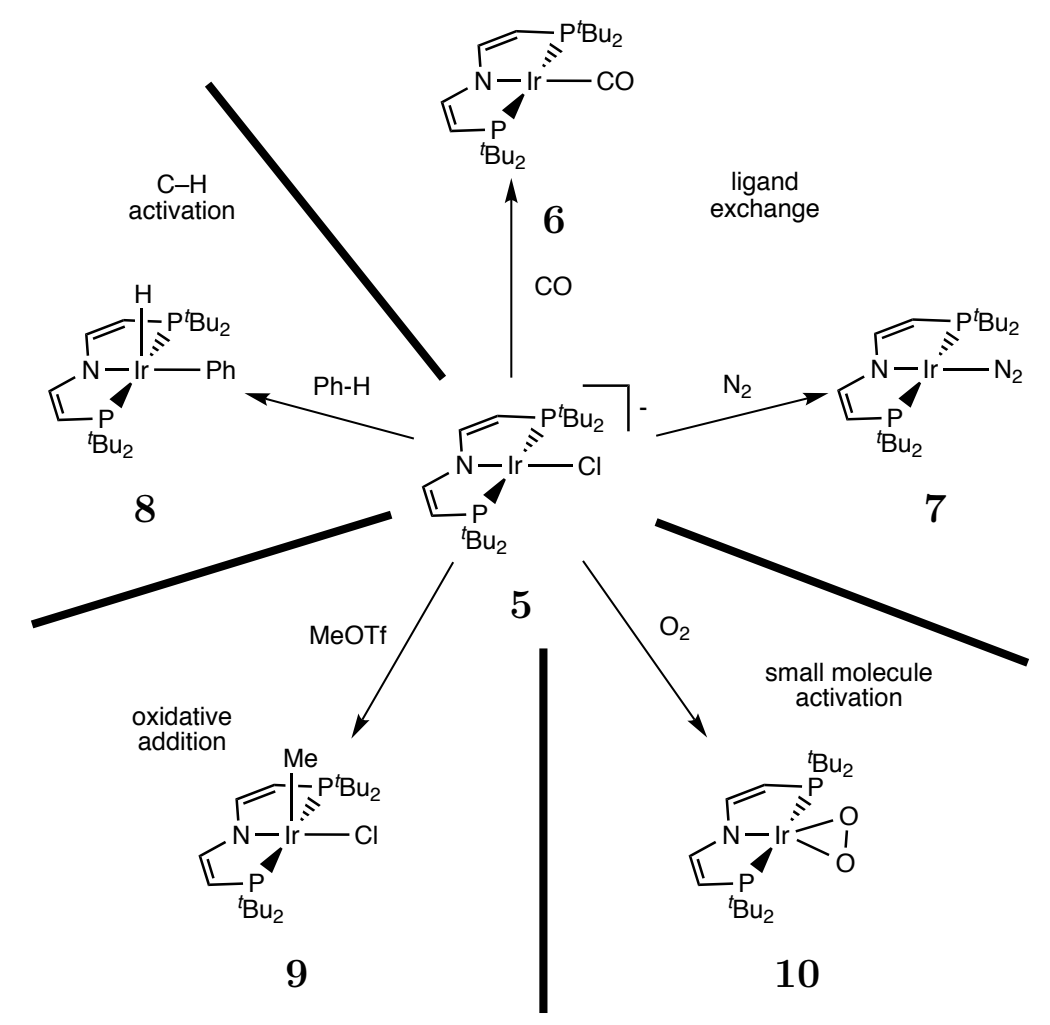

Chart III.5.2: Reactivity of square-planar Ir $^{I}$ chloro complex 5 towards ligand exchange, $C-H$ activation, oxidative addition and small molecule activation.

In addition, the versatility of divinylamido pincer ligand PNP' to stabilize a wide range of oxidation states in identical coordination environments $(\mathbf{5}, \mathbf{3}, \mathbf{4})$ and its ability to perform 1-electron redox-processes within the platinum metals is demonstrated by completion of an unusual isolated $\mathrm{Ir}^{\mathrm{I}} / \mathrm{Ir}^{\mathrm{II}} / \mathrm{Ir}^{\mathrm{III}}$ redox series. The bond parameters indicate that the extended $\pi$-system within the $\operatorname{Ir}-\mathrm{N}(-\mathrm{C}=\mathrm{C})_{2}$ moiety is a decisive electronic feature for the stability of 3, 4 and $\mathbf{5}$. While this ligand platform has similar electronic properties as the popular PNP ligands $\mathrm{N}\left(\mathrm{C}_{6} \mathrm{H}_{3} \mathrm{MePR}_{2}\right)_{2}$ and $\mathrm{N}\left(\mathrm{SiMe}_{2} \mathrm{CH}_{2} \mathrm{PR}_{2}\right)_{2}$, e.g. expressed in car- 
bonyl stretching vibrations of the corresponding $[\operatorname{Ir}(\mathrm{CO})(\mathrm{PNP})]$ complexes, it combines the hydrolytic stability of $\mathrm{N}\left(\mathrm{C}_{6} \mathrm{H}_{3} \mathrm{MePR}_{2}\right)_{2}$ (unlike $\mathrm{N}\left(\mathrm{SiMe}_{2} \mathrm{CH}_{2} \mathrm{PR}_{2}\right)_{2}$ ) with accessibility of the sterically highly protecting phosphine substituents, such as $\mathrm{P}^{t} \mathrm{Bu}_{2}$, which are on the other hand not available for $\mathrm{N}\left(\mathrm{C}_{6} \mathrm{H}_{3} \mathrm{MePR}_{2}\right)_{2} \cdot{ }^{[157]}$

The low-spin $d^{6}$ configuration of $\mathbf{4}$ is attributed to an electronic structure with one empty $\mathrm{M}-\mathrm{N} \pi$-symmetric $d$-orbital (chart III.5.1), hence, it is well suited for the stabilization of iridium element multiple bonds. However, single occupation of this orbital in the case of $\mathrm{Ir}^{\mathrm{II}}$ chloro complex 3 disfavors the formation of $\pi$-bonds and offers synthetic access to stable precursors. The possibility for electronic fine tuning based on the reversibility of the $\mathrm{Ir}^{\mathrm{II}} / \mathrm{Ir}^{\mathrm{III}}$ redox couple and the wide range of oxidation states stabilized by the electronically flexible but rigid PNP' pincer ligand qualifies these platforms for investigations on metal-element multiple bonds.

With these platforms in hand, the isolation, characterization of closed-shell iridium nitride $\left[\operatorname{Ir}(\mathrm{N})\left(\mathrm{PNP}^{\prime}\right)\right]^{+}(\mathbf{1 2})$ and spectroscopic characterization of open-shell nitrido complex $\left[\operatorname{Ir}(\mathrm{N})\left(\mathrm{PNP}^{\prime}\right)\right](\mathbf{1 3})$, as well as preliminary reactivity studies were performed (chart III.5.3). Their unusual electronic structures are characterized by strongly covalent $\mathrm{Ir} \equiv \mathrm{N}$ $\pi$-bonding and $\pi^{*}$-anti-bonding interactions. For the cationic nitrido complex 12, this leads to an effective $\mathrm{Ir}^{\mathrm{III}}$ oxidation state, which is reflected by its electrophilic nitrido ligand, as showcased by the reaction with $\mathrm{Me}_{3} \mathrm{NO}$ to produce the nitrosyl complex $\left[\operatorname{Ir}(\mathrm{NO})\left(\mathrm{PNP}^{\prime}\right)\right]^{+}(\mathbf{2 5})$. The SOMO of the neutral compound 13 essentially represents an Ir-N $\pi^{*}$-anti-bonding orbital. The high covalency of the $\mathrm{Ir}-\mathrm{N} \pi$-bond leads to an almost even distribution of the radical character over the iridium-nitrido moiety. This species is thus best described with the resonance structures $\left[\left(\mathrm{PNP}^{\prime}\right) \operatorname{Ir}^{\mathrm{IV}}{ }_{\mathrm{S}=1 / 2}\left(\mathrm{~N}^{3-}\right)_{\mathrm{S}=0}\right.$ ] and $\left[\left(\mathrm{PNP}^{\prime}\right) \mathrm{Ir}^{\mathrm{III}} \mathrm{S}=0_{0}\left(\mathrm{~N}^{\cdot 2-}\right)_{\mathrm{S}=1 / 2}\right]$ with a slightly dominating $\left\{\mathrm{Ir}=\mathrm{N}^{\cdot}\right\}$ 'nitridyl' character according to DFT. While aminyl $\left(\mathrm{R}_{2} \mathrm{~N}^{*}\right)$ and imidyl $\left(\mathrm{RN}^{--}\right)$complexes have been reported, this is the first example of an unequivocally spectroscopically characterized redox noninnocent nitrido ligand.

In accord with the decreasing steric protection within this series, $\left[\operatorname{Ir}(\mathrm{N})\left(\mathrm{PNP}^{\prime}\right)\right]$ is not thermally stable. The partial radical character of the 'nitridyl' ligand facilitates easy $\mathrm{N} \equiv \mathrm{N}$ bond formation via a bimolecular coupling process, and thus produces the binuclear radical complex $\left[\left(\mathrm{PNP}^{\prime}\right) \mathrm{Ir}^{\mathrm{I}}-\mathrm{N} \equiv \mathrm{N}-\mathrm{Ir}^{\mathrm{I}}\left(\mathrm{PNP}^{\prime}\right)\right]$ (14). Selective ammonia oxidation to $\mathrm{N}_{2}$ was established a long time ago with $\mathrm{Ru}$ and $\mathrm{Os}$ amine complexes. The experimental and theoretical bonding analysis on this new isolobal Ir system indicates that the redox non-innocence of the terminal nitrido ligand might be an important fea- 


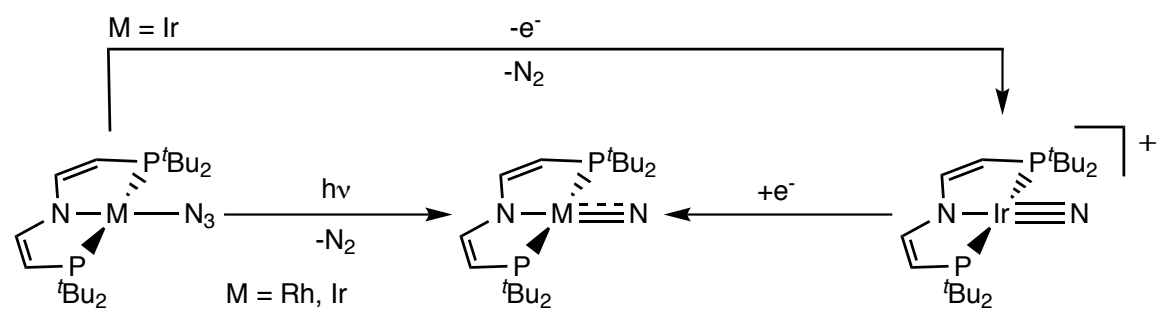

$11 / 16$

$13 / 17$
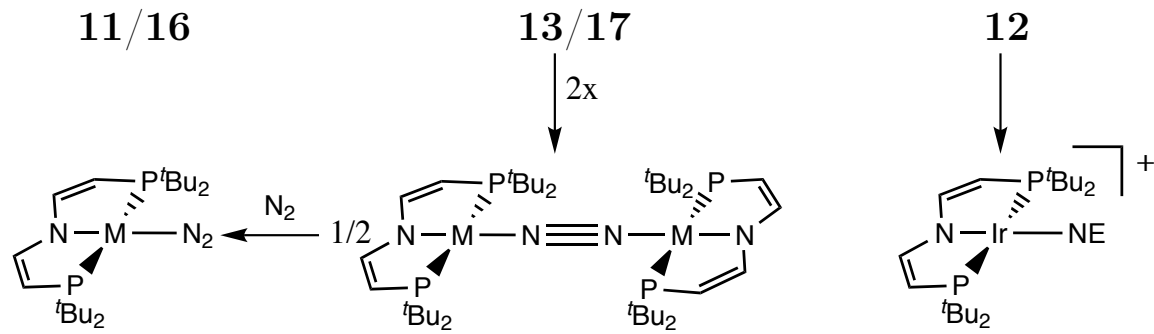

$7 / 18$

$14 / 19$

$25 / 26 / 27$

$\mathrm{E}=\mathrm{O} / \mathrm{S} / \mathrm{Se}$

Chart III.5.3: Synthesis and reactivity of nitrido complexes of rhodium and iridium.

ture to rationalize this reactivity. In view of a recent report on the reverse reaction, that is the photochemical generation of ammonia from $\mathrm{N}_{2}$ within Taube's Os complex $\left[\left(\mathrm{NH}_{3}\right)_{5} \mathrm{Os}-\mathrm{N} \equiv \mathrm{N}-\mathrm{Os}\left(\mathrm{NH}_{3}\right)_{5}\right]^{5+}$, this work might also provide a conceptual advance for nitrogen fixation. ${ }^{[25]}$

In addition, the first spectroscopic characterization of a terminal rhodium nitrido complex (17) using the same PNP' pincer ligand as established for iridium (chart III.5.3) was achieved. Analysis of the EPR data of $\mathbf{1 7}$ with the aid of DFT property calculations reveals a similar electronic structure as found for iridium. A SOMO, which is mainly localized in a nearly covalent $\mathrm{Rh}-\mathrm{N} \pi^{*}$ molecular orbital leads to substantial spin density on the nitrido ligand ( $60 \%$ ), which stresses the importance of the mesomeric structure possessing nitridyl character for the overall description of the electronic structure of $\mathbf{1 7}$. As was shown for analogous iridium nitride 13, the transient rhodium nitride $\mathbf{1 7}$ readily reacts via radical-type $\mathrm{N}-\mathrm{N}$ coupling to the corresponding dimeric $\mathrm{Rh}^{\mathrm{I}} \mathrm{N}_{2}$ complex 18. This mechanistic pathway similarly applies to $d^{3}$ nitrides, i.e. six coordinate $\mathrm{Fe}^{\mathrm{V}}$ nitrides, ${ }^{[98]}$ pointing toward a fundamental electronic structure-reactivity relationship for isolobal square-planar $\{\mathrm{M}-\mathrm{N}\}^{5}$ and octahedral $\{\mathrm{M}-\mathrm{N}\}^{3}$ complexes. Given the highly transient nature of these compounds, the selectivity of decay by $\mathrm{N}-\mathrm{N}$ coupling is remarkable even in the presence of weak $\mathrm{C}-\mathrm{H}$ bonds. The even higher nitridyl character in 
the case of Rh over Ir, which was predicted by DFT, is reflected in the lower thermal stability. Whereas detection of open-shell iridium nitrido complex $\mathbf{1 3}$ was possible in solution, the rhodium analogue is only stable in frozen solution and fast decay was also observable in the solid state at room temperature.

In addition, the synthesis of open- and closed-shell parent amido complexes of iridium $\mathbf{3}$ and 21, respectively, was demonstrated (chart III.5.4). The reactivity of amido complex 24 was elucidated in terms of conversion with $\mathrm{O}_{2}$ under formation of peroxo complex 10, protonation yielding in amino complex $\mathbf{2 0}$ and upon chemical oxidation towards cationic amido complex 21. The remarkable high thermal stability of $\mathbf{2 1}$ as an example of a rare class of diamagnetic square-planar complexes with a $d^{6}$ electron count, is attributed to the strong $\pi$-donating ability of the $\mathrm{NH}_{2}$ ligand. Estimation of the $\mathrm{N}-\mathrm{H}$ bond dissociation energies in $\mathbf{2 4}$ reveals a higher BDE of the second BDE, that is in between of 76 and $82 \mathrm{kcal} / \mathrm{mol}$, over the first $\mathrm{N}-\mathrm{H}$ bond, that is between 70 and $76 \mathrm{kcal} \mathrm{mol}^{-1}$. Attempts to isolate the parent terminal imido complex resulted in disproportionation, driven by breaking of a weak $\mathrm{N}-\mathrm{H}$ bond in the imide and formation of a strong second $\mathrm{N}-\mathrm{H}$ bond in the amide.

The transient character of open-shell iridium nitrido complex $\mathbf{1 3}$ is in contrast with the high selectivity for nitride coupling reaction, especially in the presence of weak $\mathrm{C}-\mathrm{H}$ bonds. The estimation of the relatively weak $\mathrm{N}-\mathrm{H}$ bonds ${ }^{[226]}$ determined experimentally for amido complex $\mathbf{2 4}$ and particularly the smaller BDE for the first $\mathrm{N}-\mathrm{H}$ bond over the second suggests that single HAT reaction by open-shell iridium nitride $\mathbf{1 3}$ is thermodynamically uphill. Hence, hydrogen atom abstraction of the nitride would proceed if the difference in total energy of nitride and imide is smaller than the kinetic barrier for $\mathrm{N}-\mathrm{N}$ coupling, assuming a low barrier for hydrogen atom transfer. The overall energy for hydrogen atom uptake under the formation of neutral imido complex strongly depends on the $\mathrm{E}-\mathrm{H}(\mathrm{E}=$ element $)$ bond dissociation energy of the hydrogen atom donor reagent, as demonstrated by the different reaction products upon conversion of nitride with either TEMPO-H or 2,4,6-tri-tert-butylphenol. On the other hand, a higher kinetic barrier for the abstraction of a hydrogen atom by open-shell nitride $\mathbf{1 3}$ as compared with the barrier for $\mathrm{N}-\mathrm{N}$ coupling would also explain the high selectivity for $\mathrm{N}_{2}$ formation. In this respect, selective $\mathrm{N}_{2}$ formation of $\mathbf{1 3}$ in the presence of 1,4-cyclohexadiene ( $\mathrm{BDE}_{C-H}$ (gas) $=76.0 \mathrm{kcal} / \mathrm{mol})^{[226]}$ can be rationalized.

In summary, the insertion of open-shell nitrido complexes in $\mathrm{E}-\mathrm{H}$ bonds via hydrogen atom abstraction and radical rebound, as it was demonstrated for examples for transi- 


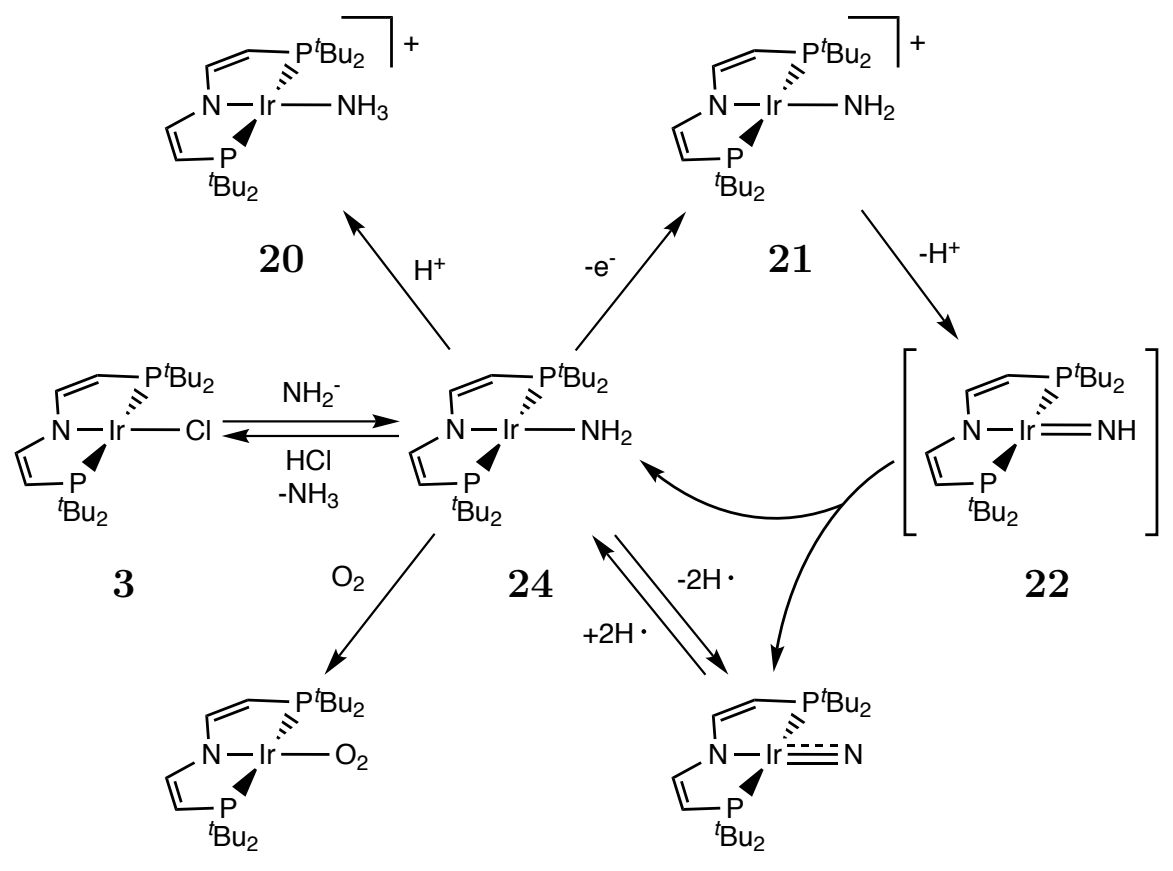

10

13

Chart III.5.4: Synthesis and reactivity of parent iridium amido complex 24.

tion metal nitrene species, is hampered by the low energy of the $\mathrm{N}-\mathrm{H}$ bond of transient imido complex and its rapid decomposition via disproportionation. Higher reactivity in terms of $\mathrm{C}-\mathrm{H}$ activation and nitrene insertion is expected for the respective alkyl and aryl imido complexes of iridium and their synthesis and reactivity studies offer interesting approaches concerning $\mathrm{N}$ group transfer reactivity.

The electrophilic reactivity of closed-shell Ir nitrido complex 12 was further explored, leading to chalcogen atom transfer either by conversion with O-atom donor reagent $\mathrm{Me}_{3} \mathrm{~N}-\mathrm{O}$ or by activation of elemental $\mathrm{S}$ and Se to afford thionitrosyl and selenonitrosyl complexes and gives rise to a rare series of chalcogenonitrosyl complexes $\left[\operatorname{Ir}(\mathrm{NE})\left(\mathrm{PNP}^{\prime}\right)\right] \mathrm{PF}_{6}$ $(\mathrm{E}=\mathrm{O}, \mathrm{S}, \mathrm{Se}$, none, chart III.5.3). The spectroscopic and crystallographic characterization of the full series allowed evaluation of current bonding models. The structural features suggest strong $\pi$-delocalization and multiple-bond character within the $\operatorname{Ir}=\mathrm{N}=\mathrm{E}$ moiety. The experimental data also supports a different model for the heavier chalcogens, which describes IrN-E bonding as a donor-acceptor interaction with a singlet chalcogen atom. However, theoretical and experimental charge density investigations are required to explain the detailed bonding within this novel series of compounds. 



\section{Part IV.}

\section{Experimental Part}




\section{Methods}

\subsection{General methods}

\section{Inert gas}

All reactions with reagents sensitive to air or moisture were performed using standard Schlenk techniques under an atmosphere of argon (99.9990\% purity, Linde gas). All glassware were pretreated in a $\mathrm{KOH} /$ Isopropanol bath, neutralized with diluted $\mathrm{HCl}$, washed with deionized water and heated to $120{ }^{\circ} \mathrm{C}$. Before utilization, the glassware was heated $i$. vac. and flushed with argon in 3 cycles. Solvents were added via steel cannulas and rubber septum or trap-to-trap transferred in the reaction vessel. Glass fiber filters (Whatman GF/B, $25 \mathrm{~mm}$ ) attached to teflon cannulas with teflon band were used for standard filtrations. A glass frit (G4) was charged with hot celite $\left(16 \mathrm{~h}\right.$ at $\left.120{ }^{\circ} \mathrm{C}\right)$ for filtration over celite. Dewar vessels cooled with Isopropanol $/ \mathrm{N}_{2}$ liq. or dry ice baths were used for low temperature synthesis. All small scale experiments $(<20 \mu \mathrm{mol})$ were performed in J-Young NMR tubes.

\section{Glove box}

Small scale reaction work up and weighing of sensitive materials was carried out in a glove box of MBraun under argon atmosphere. The atmosphere was circulated through columns filled with activated carbon, copper catalyst and molar sieves (4 $\AA$ ) to remove traces of oxygen, water and solvents.

\section{Solvents}

Pentanes, benzene, THF, toluene and DCM were passed through columns packed with activated alumina and/or molar sieves, respectively, in a solvent purification system of MBraun. Methanol, ethanol, isopropanol, acetone and DMSO were dried by stirring over 
molar sieves. Triethylamine was distilled from KOH. Acetonitrile was dried over $\mathrm{CaH}_{2}$ and distilled under argon. Deuterated solvents were dried via stirring over $\mathrm{Na} / \mathrm{K}$-alloy $\left(\mathrm{C}_{6} \mathrm{D}_{6}, d_{8}\right.$-THF, $d_{8}$-toluene) or $\mathrm{CaH}_{2}\left(\mathrm{CD}_{2} \mathrm{Cl}_{2}\right)$, respectively, for four days and degassed by three pump-freeze-thaw cycles. Separation of the solvent from drying agent was performed via trap-to-trap transfer and the solvents were stored under argon atmosphere. $d_{6}$-Acetone was dried over molar sieves for $16 \mathrm{~h}$, over $\mathrm{BO}_{3}$ for additional $16 \mathrm{~h}$ and collected by distillation under argon.

\subsection{Analytical methods}

\section{Elemental analysis}

Elemental analysis were obtained from the analytical laboratories of the Technical University Munich, the Friedrich-Alexander University Erlangen-Nuremberg (Euro EA 3000) and Georg-August University Göttingen (Elementar Vario EL 3).

\section{Magnetic measurements}

The magnetic measurements were performed using the Evans' Method modified by Sur at room temperature in $\mathrm{C}_{6} \mathrm{D}_{6} \cdot{ }^{[282,283]}$ Therefore, the sample (ca. $10 \mathrm{mg}$ ) was weighed in a J-Young NMR tube and dissolved in a defined volume (ca. $500 \mu \mathrm{L}$ ) of $\mathrm{C}_{6} \mathrm{D}_{6}$ and referenced against the signal of a sealed capillary of the same solvent. The difference in chemical shift of the residual proton resonance of the solvent and the sealed capillary was used for the calculation of the magnetic moment using equation 1.1.

$$
\chi_{\mathrm{p}}=\chi_{0}+\frac{3 \cdot \Delta \nu}{2 \cdot \pi \cdot \nu_{0} \cdot c}+\frac{\chi_{0}\left(\rho_{0}-\rho_{\mathrm{s}}\right)}{c}
$$

$\chi_{\mathrm{p}}=$ mass susceptibility of the compound $\left[\mathrm{cm}^{3} / \mathrm{g}\right]$

$\chi_{0}=$ mass susceptibility of the solvent $\left[\mathrm{cm}^{3} / \mathrm{g}\right]$

$\Delta \nu=$ difference in chemical shift of residual proton signal of the solvent versus the paramagnetic shifted signal $[\mathrm{Hz}]$

$\nu_{0}=$ spectrometer frequency

$c=$ concentration of the paramagnetic compound $\left[\mathrm{g} / \mathrm{cm}^{3}\right]$

$\rho_{0}=$ density of the pure solvent

$\rho_{\mathrm{s}}=$ density of the solution 
Rotation of the sample parallel to the magnetic field in a modern high field FT NMR spectrometer allows the modification of following Sur (s. equation 1.2).

$$
\chi_{\mathrm{p}}=\chi_{0}+\frac{3 \cdot \Delta \nu}{4 \cdot \pi \cdot \nu_{0} \cdot c}+\frac{\chi_{0}\left(\rho_{0}-\rho_{\mathrm{s}}\right)}{c}
$$

The relatively small error by disregard of the first and last term was shown by Grant ${ }^{[284]}$ and thus simplifies the equation additionally and converts the units to the SI system (s. equation 1.3).

$$
\chi_{t o t}^{M}=\frac{3 \cdot \Delta \nu}{10^{3} \cdot \nu_{0} \cdot c}
$$

$\chi_{t o t}^{M}=$ molar susceptibility of the compound $\left[\mathrm{m}^{3} / \mathrm{mol}\right]$

$\Delta \nu=$ difference in chemical shift of residual proton signal of the solvent versus the paramagnetic shifted signal [Hz]

$\nu_{0}=$ spectrometer frequency

$c \quad=$ concentration of the paramagnetic compound $\left[\mathrm{g} / \mathrm{cm}^{3}\right]$

The total molar susceptibility of the compound is adjusted by the diamagnetic contribution (s. equation 1.4).

$$
\chi_{\text {tot }}^{M}=\chi_{\text {para }}^{M}+\chi_{\text {dia }}^{M}
$$

$\chi_{\text {tot }}^{M}=$ molar total susceptibility of the compound $\left[\mathrm{m}^{3} / \mathrm{mol}\right]$

$\chi_{\text {para }}^{M}=$ molar paramagnetic susceptibility $\left[\mathrm{m}^{3} / \mathrm{mol}\right]$

$\chi_{\text {dia }}^{M}=$ molar diamagnetic susceptibility $\left[\mathrm{m}^{3} / \mathrm{mol}\right]$

The estimations of the diamagnetic susceptibilities were performed following the method of Tillieu and Guy ${ }^{[285]}$ for the ligand and of Klemm ${ }^{[286,287]}$ for the metal cations, respectively. The magnetic moment of the compound is obtained out of equation 1.5.

$$
\mu_{e f f}=\sqrt{\frac{\chi_{\text {para }}^{M} \cdot 3 \cdot k \cdot T}{\mu_{B}^{2} \cdot \mu_{0} \cdot N_{A}}}
$$




$$
\begin{aligned}
u_{e f f} & =\text { effective magnetic moment } \\
\chi_{\text {para }}^{M} & =\text { molar paramagnetic susceptibility }\left[\mathrm{m}^{3} / \mathrm{mol}\right] \\
k & =1.38066 \cdot 10^{-23} \mathrm{JK}^{-1} \text { (Boltzmann constant) } \\
\mathrm{T} & =\text { Temperatur }[\mathrm{K}] \\
\mu_{B} & =9.27402 \cdot 10^{-24} \mathrm{JT}^{-1} \text { (Bohr magneton) } \\
\mu_{0} & =4 \cdot \pi \cdot 10^{-7} \mathrm{~T}^{2} \mathrm{~m}^{3} \mathrm{~J}^{-1} \text { (magnetic field constant) } \\
N_{A} & =6.02213 \cdot 10^{23} \mathrm{~mol}^{-1} \text { (Avogadro constant) }
\end{aligned}
$$

The sample temperature was determined using the temperature unit of the spectrometer.

\section{IR spectroscopy}

IR spectra were recorded as nujol mulls between $\mathrm{KBr}$ plates or $\mathrm{KBr}$ pellets on a $J A S C O$ FT/IR-4100 spectrometer, a Bruker Vertex $70 \mathrm{FT} / \mathrm{IR}$ spectrometer, a digilab Excalibur FTS:3000, and a Thermo Scientific Nicolet iso10 spectrometer. Signal intensities are abbreviated as: vw (very weak), w (weak), m (medium), s(strong), vs (very strong).

\section{NMR spectroscopy}

NMR spectra were recorded on a Bruker Avance III 300 and 400, a Bruker Avance DRY 500 and a JEOL ECX 400 spectrometer and were calibrated to the residual proton resonance of the solvent (s. table IV.1.1). ${ }^{31} \mathrm{P}$ NMR chemical shifts relative to external phosphoric acid $(\delta=0.0 \mathrm{ppm}) .{ }^{15} \mathrm{~N}$ NMR chemical shifts are reported to external nitromethane $(\delta=0.0 \mathrm{ppm})$ and ${ }^{15} \mathrm{NH}_{4} \mathrm{NO}_{3}\left(\mathrm{CH}_{3} \mathrm{NO}_{2}\right.$ at $\left.+361 \mathrm{ppm}\right)$ for iridium nitrides and dinitrogen complexes. Signal multiplicities are abbreviated as: s (singlet), d (doublet), t (triplet), q (quartet), sept, (septet), m (multiplet), br (broad).

Table IV.1.1.: Chemical shift of residual proton resonance of deuterated solvents. [288]

\begin{tabular}{ccc}
\hline Lösemittel & \multicolumn{2}{c}{$\delta[\mathrm{ppm}]$} \\
& ${ }^{1} \mathrm{H} \mathrm{NMR}$ & ${ }^{13} \mathrm{C} \mathrm{NMR}$ \\
\hline $\mathrm{C}_{6} \mathrm{D}_{6}$ & 7.16 & 128.4 \\
$d_{8}-\mathrm{THF}$ & $3.58 ; 1.72$ & $67.2 ; 25.3$ \\
$\mathrm{CD}_{2} \mathrm{Cl}_{2}$ & 5.32 & 54.0 \\
$d_{8}$-toluene & 2.09 & 20.4 \\
\hline
\end{tabular}




\section{EPR spectroscopy}

Experimental X-band EPR spectra were recorded on a JEOL CW spectrometer JESDA200 equipped with an X-band Gunn diode oscillator bridge, a cylindrical mode cavity and a helium cryostat in cooperation with Dr. Marat Khusniyarov at Friedrich-Alexander University Erlangen-Nuremberg and with a Bruker EMX spectrometer equipped with a He temperature control cryostat system (Oxford Instruments) in cooperation with Prof. Dr. Bas de Bruin at University of Amsterdam. The spectra were simulated by iteration of the anisotropic g-values, (super)hyperfine coupling constants, and line widths using the EPR simulation program W95EPR developed buy Prof. Dr. Frank Neese.

\section{Mass spectroscopy}

Mass spectrometry was performed with a HCT ultra (ESI) and a maxis UHR-TOF mass spectrometer of Bruker.

\section{Cyclic voltammetry}

Cyclic voltammograms were either recorded with a CompactSat instrument from Ivium Technologies equipped with a platinum wire counter electrode, a platinum wire pseudoreference electrode, and a glassy carbon working electrode or a Autolab PGSTAT101 from Metrohm equipped with a platinum wire counter electrode, a platinum wire pseudoreference electrode, and a platinum working electrode. Measurements were performed in the glove box using a freshly prepared $0.1 M\left[\mathrm{~N} n \mathrm{Bu}_{4}\right] \mathrm{PF}_{6}$ solution of either THF or DCM. The ferrocene/ferrocenium couple was used as internal standard. For compounds with a oxidation potential close to $0 \mathrm{~V}\left(\mathrm{vs} . \mathrm{Fc} / \mathrm{Fc}^{+}\right.$), the decamethylferrocene/decamethylferrocenium couple was used instead as internal standard with a potential of $-0.440 \mathrm{~V}$ (vs. $\mathrm{Fc} / \mathrm{Fc}^{+}$). Further referencing was performed via a $\mathrm{Ag}$ wire in a $0.01 \mathrm{M}$ solution of $\mathrm{AgNO}_{3}$ in a $0.1 \mathrm{M}$ solution of $\left[\mathrm{N} n \mathrm{Bu}_{4}\right] \mathrm{PF}_{6}$ in THF, separated from the analytic solution via a glass diaphragm. External referencing of this electrode was performed by measurement of the reversible $\mathrm{Fc} / \mathrm{Fc}^{+}$redox couple of a $0.1 M$ solution of ferrocene in THF containing $0.1 M\left[n \mathrm{Bu}_{4} \mathrm{~N}\right] \mathrm{PF}_{6}$ (figure IV.1.1). 


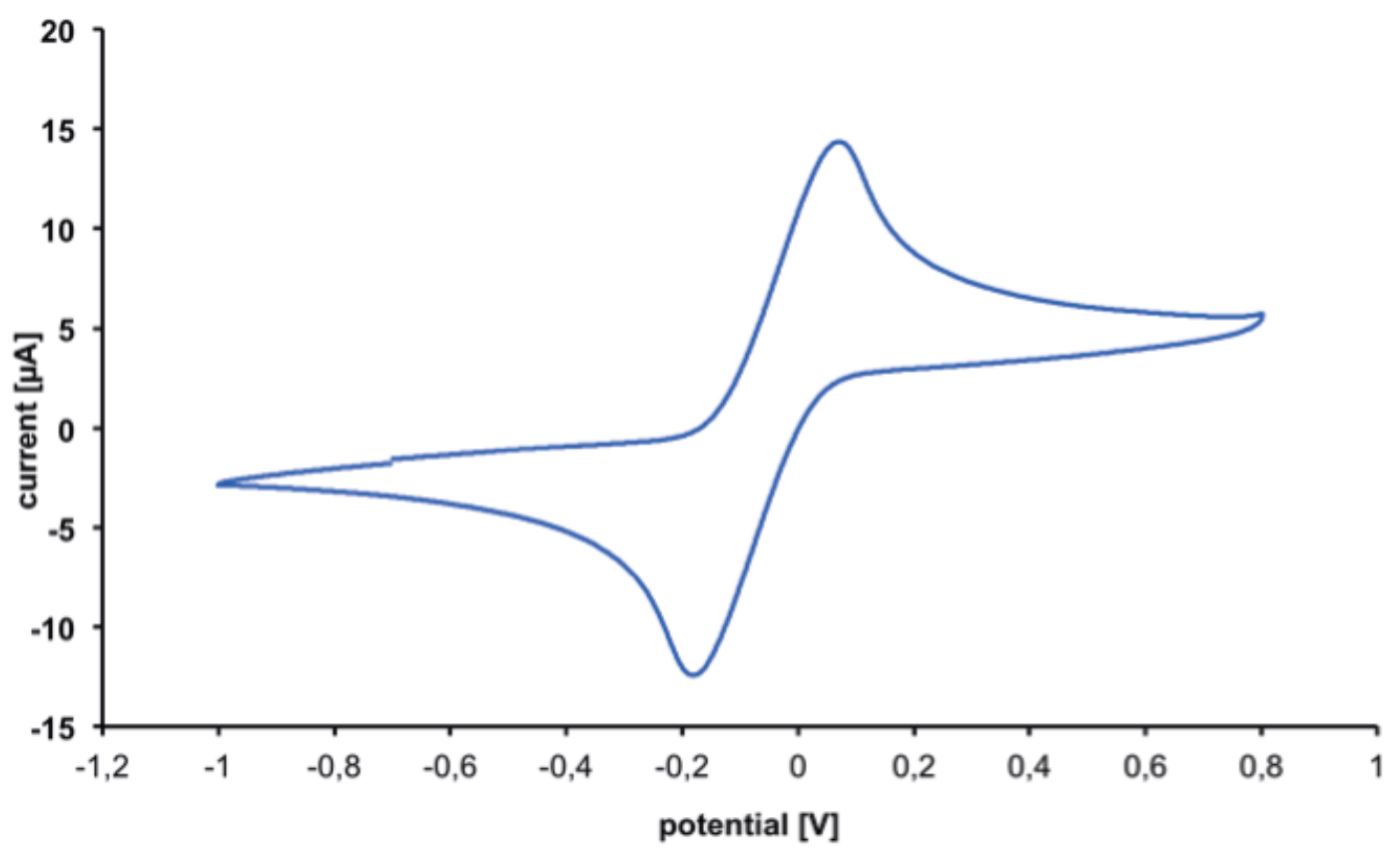

Figure IV.1.1.: Cyclic voltammogram of a $0.001 \mathrm{M}$ solution of ferrocene in THF containing $0.1 \mathrm{M} / \mathrm{nB} u_{4} N / P F_{6}$ (Pt working electrode, Pt counter electrode, $\mathrm{Ag} / \mathrm{Ag}^{+}$ reference electrode). 


\section{Starting material and ligand synthesis}

\subsection{Reagents and starting materials}

\subsubsection{Commercial available reagents and starting materials}

$\mathrm{AgPF}_{6}, \mathrm{AgSbF}_{6},\left[\mathrm{Fe}\left(\mathrm{C}_{5} \mathrm{Me}_{5}\right)\right](\mathrm{ABCR}), \mathrm{K}\left[\mathrm{N}\left(\mathrm{SiMe}_{3}\right)_{2}\right]$, Di-tert-butylchlorophosphine (Alfa), Sulfur, KF, $\mathrm{NaN}_{3}$ (Merck), $\left[\mathrm{Co}\left(\mathrm{C}_{5} \mathrm{Me}_{5}\right)_{2}\right]$, $\left[\mathrm{Fe}\left(\mathrm{C}_{5} \mathrm{H}_{5}\right)\right]$, Lithium granula, Selenium (Riedel- de Haen), TEMPO, $\mathrm{CH}_{3} \mathrm{SO}_{3} \mathrm{CF}_{3}, \mathrm{NaNH}_{2}$, bis(triphenylphosphoranylidene)ammonium chloride, methyl triflate, $\mathrm{KBr}$ (Sigma-Aldrich), $\mathrm{C}_{10} \mathrm{H}_{10} \mathrm{O}_{5}$, 2,4,6-Tritert-butylphenol, and $\left[\mathrm{Fe}\left(\mathrm{C}_{5} \mathrm{H}_{5}\right)_{2}\right] \mathrm{PF}_{6}$ (Acros) were used as purchased. $\mathrm{Na}^{15} \mathrm{~N}^{14} \mathrm{~N}_{2}$ was purchased from Cambridge Isotopic Laboratories. 1,4-Benzoquinone and $\mathrm{Me}_{3} \mathrm{NO}$ (Merck) were sublimed in prior to use. Silica gel 60 silanized was purchased from Merck and heated $i$. vac. for 16 hours prior to use. 1,4-Cyclohexadiene was purchased from Alfa Aesar, dried over $\mathrm{CaH}_{2}$, and distilled prior to use. $\mathrm{O}_{2}$ and $\mathrm{CO}$ (Linde gas) were used as purchased. $\mathrm{H}_{2}$ (Linde gas) was dried over a column of $\mathrm{P}_{2} \mathrm{O}_{5}$.

\subsubsection{Non-commercial available reagents and starting materials}

Following reagents and starting materials were not obtained commercially and synthesized following literature: 


\begin{tabular}{ll}
\hline Compound & Literature \\
\hline $\mathrm{Di}(2$-chloroethyl)trimethylsilylamine & {$[289]$} \\
$\mathrm{Di}($ cis-cyclooctene)iridium(I)chloro-dimer & {$[290]$} \\
$\mathrm{Bis}($ triphenylphosphoranylidene)ammonium azide & {$[291]$} \\
$\mathrm{KC}_{8}$ & {$[292]$} \\
$\mathrm{Tris}($ triphenylphosphine)chlororhodium $(\mathrm{I})$ & {$[293]$} \\
$\mathrm{Na} / \mathrm{Hg}$ & {$[294]$} \\
$\mathrm{H}\left[\mathrm{B}\left\{\mathrm{C}_{6} \mathrm{H}_{3}\left(\mathrm{CF}_{3}\right)_{2}\right\}_{4}\right]$ & {$[295]$} \\
$\mathrm{TEMPO}-\mathrm{H}$ & {$[296]$} \\
$2,4,6$-tri-tert-butylphenoxyl radical & {$[297]$} \\
\hline
\end{tabular}

\subsection{Modified procedures}

\subsubsection{Bis(di-tert-butylphosphinoethyl)amine (1)}

A procedure for the synthesis of compound $\mathbf{1}$ is already described, however, the improved synthetic protocol described here facilitates its isolation by reduction of the number of steps. ${ }^{[162]}$

A solution of ${ }^{t} \mathrm{Bu}_{2} \mathrm{PCl}(3.5 \mathrm{~mL} ; 3.35 \mathrm{~g} ; 18.54 \mathrm{mmol} ; 2 \mathrm{eq})$ in THF $(10 \mathrm{~mL})$ is added drop wise to a suspension of lithium granular (0.386 g; $55.62 \mathrm{mmol} ; 6 \mathrm{eq})$ in THF (25 mL) at $0{ }^{\circ} \mathrm{C}$ over a period of $30 \mathrm{~min}$. The solution is warmed slowly to ambient temperatures and stirred at room temperature for $4 \mathrm{~d}$. The remaining lithium is decanted off and a solution of di-(chloroethyl)trimethylsilylamine (1.985 g; $9.27 \mathrm{mmol}$; $1 \mathrm{eq})$ in THF (10 mL) is added drop wise over a period of 30 minutes at $-50{ }^{\circ} \mathrm{C}$. The solution is slowly warmed to ambient temperatures and heated to $70{ }^{\circ} \mathrm{C}$ for $16 \mathrm{~h}$. After cooling to room temperature, a solution of potassium fluoride $(0.986 \mathrm{~g} ; 16.96 \mathrm{mmol} ; 1.83 \mathrm{eq})$ in $\mathrm{MeOH}(30 \mathrm{~mL})$ is added at $0{ }^{\circ} \mathrm{C}$ and stirred for $1.5 \mathrm{~h}$. After standing for $16 \mathrm{~h}$ at room temperature, the solution is filtered off, the residue is extracted with THF $(10 \mathrm{~mL})$ and all volatiles are removed $i$. vac. The crude product is extracted with pentanes and after removal the solvent, $\left({ }^{t} \mathrm{Bu}_{2} \mathrm{PCH}_{2} \mathrm{CH}_{2}\right)_{2} \mathrm{NH}(\mathbf{1})(2.15 \mathrm{~g} ; 5.95 \mathrm{mmol} ; 65 \%)$ is obtained as a yellow oil in a spectroscopic purity of $85 \%$ based on ${ }^{1} \mathrm{H}$ NMR Integration. 


\section{Spectroscopic Characterization}

$\begin{array}{ll}{ }^{1} \mathrm{H} N M R: \quad & \left(250 \mathrm{MHz}, \mathrm{C}_{6} \mathrm{D}_{6}, \mathrm{RT}\right): \delta=2.90\left(\mathrm{td},{ }^{3} J_{\mathrm{HH}}=7.5 \mathrm{~Hz},\right. \\ & \left.{ }^{3} J_{\mathrm{HP}}=15 \mathrm{~Hz}, 4 \mathrm{H}, \mathrm{NCH}{ }_{2} \mathrm{CH}_{2} \mathrm{P}\right), 1.59\left(\mathrm{dt},{ }^{3} J_{\mathrm{HH}}=7.5\right. \\ & \left.\mathrm{Hz},{ }^{2} J_{\mathrm{HP}}=3.9 \mathrm{~Hz}, 4 \mathrm{H}, \mathrm{NCH}_{2} \mathrm{C} H_{2} \mathrm{P}\right), 1.08\left(\mathrm{~d},{ }^{3} J_{\mathrm{HP}}=\right. \\ & \left.10.8 \mathrm{~Hz}, 36 \mathrm{H}, \mathrm{P}\left(\mathrm{C}(\mathrm{CH})_{3}\right)_{2}\right) . \\ & \left(100.62 \mathrm{MHz}, \mathrm{C}_{6} \mathrm{D}_{6}, \mathrm{RT}\right): \delta=51.02\left(\mathrm{~d},{ }^{2} J_{\mathrm{CP}}=30.3 \mathrm{~Hz},\right. \\ & \left.\mathrm{N} C \mathrm{H}_{2} \mathrm{CH}_{2} \mathrm{P}\right), 31.2\left(\mathrm{~d},{ }^{1} J_{\mathrm{CP}}=22.0 \mathrm{~Hz}, \mathrm{P}\left(C\left(\mathrm{CH}_{3}\right)_{3}\right)_{2}\right), \\ & 29.9\left(\mathrm{~d},{ }^{2} J_{\mathrm{CP}}=13.9 \mathrm{~Hz}, \mathrm{P}\left(\mathrm{C}\left(C \mathrm{H}_{3}\right)_{3}\right)_{2}\right), 23.0\left(\mathrm{~d},{ }^{1} J_{\mathrm{CP}}=\right. \\ & \left.22.8 \mathrm{~Hz}, \mathrm{NCH}_{2} C \mathrm{H}_{2} \mathrm{P}\right) . \\ & \left(101.25 \mathrm{MHz}, \mathrm{C}_{6} \mathrm{D}_{6}, \mathrm{RT}\right): \delta=21.6(\mathrm{~s}) .\end{array}$

\subsubsection{Bis(triphenylphosphoranylidene)ammonium azide}

A solution of $\mathrm{NaN}_{3}(0.100 \mathrm{~g} ; 1.5 \mathrm{mmol} ; 1 \mathrm{eq})$ in $\mathrm{H}_{2} \mathrm{O}(0.5 \mathrm{~mL})$ is added to a solution obtained after heating a suspension of $\left[\left(\mathrm{Ph}_{3} \mathrm{P}\right)_{2} \mathrm{~N}\right] \mathrm{Cl}(0.883 \mathrm{~g} ; 1.5 \mathrm{mmol} ; 1$ eq $)$ in $\mathrm{H}_{2} \mathrm{O}$ $(5 \mathrm{~mL})$ for $5 \mathrm{~min}$ at $70{ }^{\circ} \mathrm{C}$ and is stirred for additional $5 \mathrm{~min}$. Afterwards, the product is precipitated at $0{ }^{\circ} \mathrm{C}$ for $1 \mathrm{~h}$, collected by filtration and extracted with MeCN $(3 \times 10 \mathrm{~mL})$. The solution is concentrated and precipitation of the product is initiated by addition of $\mathrm{Et}_{2} \mathrm{O}(50 \mathrm{~mL})$. After crystallization for $16 \mathrm{~h}$ at $-30{ }^{\circ} \mathrm{C}$, the crystals are collected by filtration, washed with $\mathrm{Et}_{2} \mathrm{O}(2 \times 5 \mathrm{~mL})$ and $\left[\left(\mathrm{Ph}_{3} \mathrm{P}\right)_{2} \mathrm{~N}\right] \mathrm{N}_{3}$ is obtained as colorless crystalline solid (yield: $0.530 \mathrm{~g} ; 0.91 \mathrm{mmol} ; 59 \%$ ) 


\section{Iridium(I-III) PNP plattforms}

\section{1. $\left[\operatorname{lr}\left\{\mathrm{N}\left(\mathrm{CHCHP}{ }^{t} \mathrm{Bu}_{2}\right)_{2}\right\} \mathrm{Cl}\right](3)$}

\section{Published route}

A solution of $\mathrm{NH}\left(\mathrm{CH}_{2} \mathrm{CH}_{2} \mathrm{P}^{t} \mathrm{Bu}_{2}\right)_{2}(489 \mathrm{mg} ; 1.36 \mu \mathrm{mol} ; 1 \mathrm{eq})$ in THF $(5 \mathrm{~mL})$ is added to a suspension of $\left[\operatorname{Ir}(\mathrm{COE})_{2} \mathrm{Cl}\right]_{2}(596 \mathrm{mg} ; 665 \mu \mathrm{mol} ; 0.5 \mathrm{eq})$ in THF $(25 \mathrm{~mL})$ and stirred at room temperature. After $15 \mathrm{~min}$, a solution of para-benzoquinone (376 mg; $3.48 \mu \mathrm{mol} ; 2.5 \mathrm{eq})$ in THF $(10 \mathrm{~mL})$ is added drop wise and the mixture is stirred for $16 \mathrm{~h}$ at room temperature. The solvent is removed $i$. vac. and the residue is extracted with toluene/pentanes 1:1 $(3 \times 10 \mathrm{~mL})$. The solvent is concentrated $i$. vac. and crystallized at $-18{ }^{\circ} \mathrm{C}$ for $16 \mathrm{~h} .3(318 \mathrm{mg} ; 544 \mu \mathrm{mol} ; 41 \%)$ is obtained as turquoise crystals.

\section{Elemental Analysis}

$\mathrm{C}_{20} \mathrm{H}_{40} \mathrm{ClIrNP}_{2}$ (584.16) calcd.: C, 41.12; H, 6.90; N, 2.40.

found: C, 41.09; H, 7.11; N, 2.44.

\section{Mass Spectrometric Characterization}

FAB-MS

$$
\begin{aligned}
& m / z:=584.1\left([\mathrm{M}]^{+}, 100 \%\right), \quad 528.1\left(\left[\mathrm{M}-\mathrm{CH}_{2} \mathrm{CMe}_{2}\right]^{+},\right. \\
& 39 \%), 472.0\left(\left[\mathrm{M}-2 \mathrm{CH}_{2} \mathrm{CMe}_{2}\right]^{+}, 43 \%\right) .
\end{aligned}
$$




\section{Spectroscopic Characterization}

${ }^{1} H-N M R$ :

$\left(250 \mathrm{MHz}, \mathrm{C}_{6} \mathrm{D}_{6}, \mathrm{RT}\right): \delta=10.45\left(\mathrm{br}, 36 \mathrm{H}, \mathrm{P}\left(\mathrm{C}\left(\mathrm{CH}_{3}\right)_{3}\right)_{2}\right)$, -6.77 (br, 2H, CH) , -138.17 (br, $2 \mathrm{H}, \mathrm{CH}$ ).

\section{Improved synthesis}

A solution of $\mathrm{NH}\left(\mathrm{CH}_{2} \mathrm{CH}_{2} \mathrm{P}^{t} \mathrm{Bu}_{2}\right)_{2}(1.21 \mathrm{~g} ; 3.35 \mathrm{mmol} ; 2 \mathrm{eq})$ in THF $(10 \mathrm{~mL})$ is added to a suspension of $\left[\operatorname{Ir}(\mathrm{COE}){ }_{2} \mathrm{Cl}\right]_{2}(1.50 \mathrm{~g} ; 1.67 \mathrm{mmol} ; 1 \mathrm{eq})$ in THF $(15 \mathrm{~mL})$ and stirred at room temperature. After $15 \mathrm{~min}$, a solution of para-benzoquinone $(0.91 \mathrm{~g} ; 8.37 \mathrm{mmol}$; $5 \mathrm{eq})$ in THF $(15 \mathrm{~mL})$ is added drop wise and the mixture stirred for $16 \mathrm{~h}$ at room temperature. The suspension is extracted with THF $(3 \times 10 \mathrm{~mL})$ and after removal of all volatiles, the residue is extracted with benzene $(8 \times 10 \mathrm{~mL})$ and the solvent is removed $i$. vac. Afterwards, the crude product is extracted with pentanes $(10 \times 20 \mathrm{~mL})$, and after removal of the solvent to dryness, the remaining solid is lyophilized out of benzene $(20 \mathrm{~mL})$ and sublimed $\left(60{ }^{\circ} \mathrm{C}, 30 \mathrm{mTorr}, 1 \mathrm{~h}\right)$. After lyophilization of the residue out of benzene (20 mL), 3 (1.42 g; $2.43 \mathrm{mmol} ; 73 \%$ ) is obtained as fine turquoise powder.

\section{Elemental Analysis}

$\mathrm{C}_{20} \mathrm{H}_{40} \mathrm{ClIrNP}_{2}(584.16) \quad$ calcd.: C, 41.12; H, 6.90; N, 2.40.

found: C, 42.60; H, 7.15; N, 2.20.

\section{2. $\left[\operatorname{Ir}\left\{\mathrm{N}\left(\mathrm{CHCHP}^{t} \mathrm{Bu}_{2}\right)_{2}\right\} \mathrm{Cl}\right] \mathrm{PF}_{6}$ (4)}

A mixture of $3(1.3 \mathrm{mg} ; 19.3 \mu \mathrm{mol} ; 1.1 \mathrm{eq})$ and $\mathrm{AgPF}_{6}(4.9 \mathrm{mg} ; 19 \mu \mathrm{mol} ; 1 \mathrm{eq})$ is dissolved in cold THF $(0.5 \mathrm{~mL})$ and stirred for 5 minutes at $-60{ }^{\circ} \mathrm{C}$. The solvent is removed $i$. vac., the residue is washed with pentanes $(3 \times 0.5 \mathrm{~mL})$, rapidly extracted with THF $(3 \times 1 \mathrm{~mL})$ at room temperature and filtered into a cooled flask at $-60{ }^{\circ} \mathrm{C}$. After removal of the solvent, the residue is washed with toluene $(3 \times 0.5 \mathrm{~mL})$ and dried i. vac. The product ( $5.5 \mathrm{mg} ; 7.5 \mu \mathrm{mol} ; 39 \%$ ) is isolated as a temperature sensitive black powder. 


\section{Elemental Analysis}

$\mathrm{C}_{20} \mathrm{H}_{40} \mathrm{ClF}_{6} \mathrm{IrNP}_{3}$

Ber.: C, 33.60; H, 5.64; N, 1.88 .

$\times 0.2 \mathrm{THF}(729.12)$

Gef.: C, 34.08; H, 5.88; N, 1.78 .

\section{Spectroscopic Characterization}

${ }^{1} \mathrm{H}-\mathrm{NMR}: \quad\left(400.13 \mathrm{MHz}, \mathrm{CD}_{2} \mathrm{Cl}_{2}, \mathrm{RT}\right): \delta=4.70$ (ABXX'B'A', $N=\left|{ }^{2} J_{\mathrm{HP}}+{ }^{4} J_{\mathrm{HP}}\right|=16.3 \mathrm{~Hz},{ }^{3} J_{\mathrm{HH}}=5.5 \mathrm{~Hz}, 2 \mathrm{H}$, $\mathrm{NCHCHP}), 2.34\left(\mathrm{~A}_{18} \mathrm{XX}^{\prime} \mathrm{A}^{\prime}{ }_{18}, N=\left|{ }^{3} J_{\mathrm{HP}}+{ }^{5} J_{\mathrm{HP}}\right|=\right.$ $\left.7.8 \mathrm{~Hz}, 36 \mathrm{H}, \mathrm{P}\left(\mathrm{C}\left(\mathrm{CH}_{3}\right)_{3}\right)_{2}\right), 1.8\left(\mathrm{t},{ }^{3} J_{\mathrm{HP}}=7 \mathrm{~Hz}, 2 \mathrm{H}\right.$, $\mathrm{NCHCHP})$.

${ }^{31} P\left\{{ }^{1} H\right\}-N M R: \quad\left(101.25 \mathrm{MHz}, \mathrm{C}_{2} \mathrm{Cl}_{2}, \mathrm{RT}\right): \delta=7.8\left(\mathrm{~s}, P\left(\mathrm{C}\left(\mathrm{CH}_{3}\right)_{3}\right)_{2}\right),-145$ (hept, ${ }^{1} J_{\mathrm{PF}}=711 \mathrm{~Hz}, P \mathrm{~F}_{6}$ ).

\section{3. $\left[\mathrm{K}\left(\mathrm{C}_{10} \mathrm{H}_{10} \mathrm{O}_{5}\right)_{2}\right]\left[\operatorname{Ir}\left\{\mathrm{N}\left(\mathrm{CHCHP}^{t} \mathrm{Bu}_{2}\right)_{2}\right\} \mathrm{Cl}\right](5)$}

A mixture of 3 (200.0 mg; $342.4 \mu \mathrm{mol} ; 1 \mathrm{eq})$ and $\mathrm{KC}_{8}(55.5 \mathrm{mg} ; 410.8 \mu \mathrm{mol} ; 1.2 \mathrm{eq})$ is dissolved in a solution of $\mathrm{C}_{10} \mathrm{H}_{10} \mathrm{O}_{5}(164.0 \mu \mathrm{L} ; 821.7 \mu \mathrm{mol} ; 2.4 \mathrm{eq})$ in THF $(15 \mathrm{~mL})$ at $-50{ }^{\circ} \mathrm{C}$. After 5 minutes of stirring, all volatiles are removed $i$. vac. and the residue is extracted with THF $(3 \times 7 \mathrm{~mL})$, layered with pentanes and crystallized at $-32{ }^{\circ} \mathrm{C}$ overnight. The crystals are collected by filtration, washed with pentanes $(2 \times 5 \mathrm{~mL})$ and dried $i$. vac. $\mathbf{5}$ is obtained as an orange microcrystalline solid (yield: $164.0 \mathrm{mg}$; $154.2 \mu \mathrm{mol} ; 45 \%)$.

\section{Elemental Analysis}

$\mathrm{C}_{40} \mathrm{H}_{80} \mathrm{ClIrKNO}_{10} \mathrm{P}_{2}$ (1063.69) calcd: $\mathrm{C}, 45.16 ; \mathrm{H}, 7.58 ; \mathrm{N}, 1.32$.

found: $\mathrm{C}, 44.97 ; \mathrm{H}, 7.60 ; \mathrm{N}, 1.29$. 


\section{Spectroscopic Characterization}

${ }^{1} H-N M R:$

$\left(300 \mathrm{MHz}, d_{\delta^{-}}\right.$THF, RT) $\delta=6.93$ (ABXX'B'A', $N$ $=\left|{ }^{2} J_{\mathrm{HP}}+{ }^{4} J_{\mathrm{HP}}\right|=17.2 \mathrm{~Hz},{ }^{3} J_{\mathrm{HH}}=5.2 \mathrm{~Hz}, 2 \mathrm{H}$, $\mathrm{NCHCHP}$ ), 4.04 (ABXX'B'A', $N=\left|{ }^{2} J_{\mathrm{HP}}+{ }^{4} J_{\mathrm{HP}}\right|=$ $\left.4.3 \mathrm{~Hz},{ }^{3} J_{\mathrm{HH}}=4.3 \mathrm{~Hz}, 2 \mathrm{H}, \mathrm{NCHCHP}\right), 3.63(\mathrm{~s}, 40 \mathrm{H}$, $\left.\left(\mathrm{CH}_{2} \mathrm{CH}_{2} \mathrm{O}\right)_{5}\right), 1.35\left(\mathrm{~A}_{18} \mathrm{XX}^{\prime} \mathrm{A}^{\prime}{ }_{18}, N=\left|{ }^{3} J_{\mathrm{HP}}+{ }^{5} J_{\mathrm{HP}}\right|=\right.$ $\left.5.9 \mathrm{~Hz}, 36 \mathrm{H}, \mathrm{P}\left(\mathrm{C}\left(\mathrm{CH}_{3}\right)_{3}\right)_{2}\right)$.

${ }^{1} H-N M R$ :

$\left(75 \mathrm{MHz}, d_{8}\right.$-THF, RT): $\delta=122.4$ (AXX'A', $N=$ $\left|{ }^{2} J_{\mathrm{CP}}+{ }^{3} J_{\mathrm{CP}}\right|=5.8 \mathrm{~Hz}, \mathrm{NCHCHP}$ ), 95.0 (AXX'A', $\left.N=\left|{ }^{1} J_{\mathrm{CP}}+{ }^{3} J_{\mathrm{CP}}\right|=22.5 \mathrm{~Hz}, \mathrm{NCHCHP}\right), 68.6(\mathrm{~s}$, $\left.\left(\mathrm{CH}_{2} \mathrm{CH}_{2} \mathrm{O}\right)_{5}\right), 38.8\left(\mathrm{~A}_{2} \mathrm{XX}^{\prime} \mathrm{A}^{\prime}{ }_{2}, N=\left|{ }^{1} J_{\mathrm{CP}}+{ }^{3} J_{\mathrm{CP}}\right|=\right.$ $\left.11.2 \mathrm{~Hz}, \mathrm{P} C\left(\mathrm{CH}_{3}\right)_{3}\right), 29.9\left(\mathrm{~A}_{6} \mathrm{XX}^{\prime} \mathrm{A}_{6}^{\prime}, N=\left|{ }^{2} J_{\mathrm{CP}}+{ }^{4} J_{\mathrm{CP}}\right|\right.$ $\left.=1.7 \mathrm{~Hz}, \mathrm{PC}\left(\mathrm{CH}_{3}\right)_{3}\right)$.

${ }^{31} P\left\{{ }^{1} H\right\}-N M R: \quad\left(121 \mathrm{MHz}, d_{8^{-T H F}}, \mathrm{RT}\right): \delta=55.5(\mathrm{~s})$. 


\section{Small molecule activation by iridium(I) PNP}

\section{1. $\left[\operatorname{Ir}\left\{\mathrm{N}\left(\mathrm{CHCHP}^{t} \mathrm{Bu}_{2}\right)_{2}\right\}(\mathrm{CO})\right](6)$}

A mixture of 3 (60.0 mg; $102.7 \mu \mathrm{mol} ; 1.0 \mathrm{eq})$ and $\mathrm{KC}_{8}(13.9 \mathrm{mg} ; 102.7 \mu \mathrm{mol} ; 1.0 \mathrm{eq})$ is dissolved at $-50{ }^{\circ} \mathrm{C}$ in a THF solution $(15 \mathrm{~mL})$ saturated with $\mathrm{CO}$ and stirred for $15 \mathrm{~min}$. After removal of all volatiles $i$. vac., the residue is extracted with pentanes $(3 \times 4 \mathrm{~mL})$ and after evaporation to dryness, the crude product is purified by column chromatography (silanized silica gel, $0.3 \times 6 \mathrm{~cm}$, pentanes). After removing the solvent $i$. vac., the product is lyophilized out of benzene $(5 \mathrm{~mL})$ and 6 is obtained as bright yellow powder (30.4 mg; $58.9 \mu \mathrm{mol} ; 51 \%$ ).

\section{Elemental Analysis}

$\mathrm{C}_{21} \mathrm{H}_{40} \mathrm{NOP}_{2} \mathrm{Ir}(576.71) \quad$ calcd.: C, 43.73; H, 6.99; N, 2.43 .

found: C, 43.82; H, 7.01; N, 2.16 .

\section{Spectroscopic Characterization}

${ }^{1} H$-NMR: $\quad\left(400 \mathrm{MHz}, \mathrm{C}_{6} \mathrm{D}_{6}, \mathrm{RT}\right): \delta=7.02\left(\mathrm{ABXX}^{\prime} \mathrm{B}^{\prime} \mathrm{A} ', N=\left.\right|^{3} J_{\mathrm{HP}}\right.$ $\left.+{ }^{4} J_{\mathrm{HP}} \mid=18.9 \mathrm{~Hz},{ }^{3} J_{\mathrm{HH}}=5.8 \mathrm{~Hz}, 2 \mathrm{H}, \mathrm{NCHCHP}\right), 4.31$ $\left(\mathrm{ABXX}^{\prime} \mathrm{B}^{\prime} \mathrm{A},, N=\left.\right|^{2} J_{\mathrm{HP}}+{ }^{4} J_{\mathrm{HP}} \mid=3.4 \mathrm{~Hz},{ }^{3} J_{\mathrm{HH}}=\right.$ $5.5 \mathrm{~Hz}, 2 \mathrm{H}, \mathrm{NCHCHP}), 1.36\left(\mathrm{~A}_{18} \mathrm{XX}^{\prime} \mathrm{A}^{\prime}{ }_{18}, N=\mid{ }^{3} J_{\mathrm{HP}}+\right.$ $\left.{ }^{5} J_{\mathrm{HP}} \mid=7.0 \mathrm{~Hz}, 36 \mathrm{H}, \mathrm{P}\left(\mathrm{C}\left(\mathrm{CH}_{3}\right)_{3}\right)_{2}\right)$. 


$\begin{array}{ll}{ }^{13} C\left\{{ }^{1} H\right\}-N M R: & \left(101.25 \mathrm{MHz}, \mathrm{C}_{6} \mathrm{D}_{6}, \mathrm{RT}\right): \delta=190.6\left(\mathrm{t}^{2}{ }^{2} J_{\mathrm{CP}}=7.6,\right. \\ & \mathrm{Ir}-C \mathrm{O}), 163.7\left(\mathrm{AXX} \mathrm{XX}^{\prime}, N=\left|{ }^{2} J_{\mathrm{CP}}+{ }^{3} J_{\mathrm{CP}}\right|=9.6 \mathrm{~Hz},\right. \\ & \mathrm{N} C \mathrm{HCHP}), 85.8 \quad\left(\mathrm{AXX} \mathrm{A}^{\prime}, N=\left|{ }^{1} J_{\mathrm{CP}}+{ }^{3} J_{\mathrm{CP}}\right|=\right. \\ & 21.5 \mathrm{~Hz}, \mathrm{NCHCHP}), 36.7\left(\mathrm{~A}_{2} \mathrm{XX}^{\prime} \mathrm{A}_{2}, N=\mid{ }^{1} J_{\mathrm{CP}}+\right. \\ & \left.{ }^{3} J_{\mathrm{CP}} \mid=13.5 \mathrm{~Hz}, \mathrm{P}\left(C\left(\mathrm{CH}_{3}\right)_{3}\right)_{2}\right), 29.8\left(\mathrm{~A}_{6} \mathrm{XX}^{\prime} \mathrm{A}^{\prime}{ }_{6}, N=\right. \\ & \left.{ }^{2} J_{\mathrm{CP}}+{ }^{4} J_{\mathrm{CP}} \mid=3.0 \mathrm{~Hz}, \mathrm{P}\left(\mathrm{C}\left(C \mathrm{H}_{3}\right)_{3}\right)_{2}\right) . \\ & \left(161.93 \mathrm{MHz}, \mathrm{C}_{6} \mathrm{D}_{6}, \mathrm{RT}\right): \delta=82.0(\mathrm{~s}) . \\ & (\text { Nujol-mull/KBr}) \nu=1937 \text { vs }(\nu(\mathrm{CO})) .\end{array}$

\section{2. $\left[\operatorname{Ir}\left\{\mathrm{N}\left(\mathrm{CHCHP}^{t} \mathrm{Bu}_{2}\right)_{2}\right\}(\mathrm{H})\left(\mathrm{C}_{6} \mathrm{H}_{5}\right)\right](8)$}

A solution of $3(30.0 \mathrm{mg} ; 51.4 \mu \mathrm{mol} ; 1.0 \mathrm{eq})$ in benzene $(5 \mathrm{~mL})$ is added to $\mathrm{Na} / \mathrm{Hg}$ ( $1 \mathrm{~mol} / \mathrm{L} ; 833.4 \mathrm{mg} ; 61.6 \mu \mathrm{mol} ; 1.2 \mathrm{eq}$ ) and stirred at room temperature for $16 \mathrm{~h}$. The solution is decanted off, the $\mathrm{Hg}$ slurry is extracted with benzene $(2 \times 5 \mathrm{~mL})$ and the combined organic fractions are filtered over a pad of celite. After the removal of all volatiles $i$. vac., the crude product is extracted with pentanes $(2 \times 5 \mathrm{~mL})$, concentrated and crystallized at $-82{ }^{\circ} \mathrm{C}$. The crystals are collected by filtration, washed with cold pentanes $(3 \mathrm{~mL})$, dissolved in benzene $(7 \mathrm{~mL})$ and lyophilized overnight. 8 is obtained as red powder (13.0 mg; $21.2 \mu \mathrm{mol} ; 41 \%$ ).

\section{Elemental Analysis}

$\mathrm{C}_{26} \mathrm{H}_{46} \mathrm{NP}_{2} \operatorname{Ir}(626.83)$

calcd.: C, 49.82; H, 7.40; N, 2.23.

found: C, 49.42; H, 7.25; N, 2.15. 


\section{Spectroscopic Characterization}

${ }^{1} H-N M R$ :

$\left(300 \mathrm{MHz}, \mathrm{C}_{6} \mathrm{D}_{6}, \mathrm{RT}\right): \delta=7.73\left(\mathrm{~d},{ }^{3} J_{\mathrm{HH}}=7.5 \mathrm{~Hz}, 2 \mathrm{H}\right.$, ortho- $\mathrm{C}_{6} \mathrm{H}_{5}$ ), 7.37 (ABXX'B'A', $N=\left|{ }^{3} J_{\mathrm{HP}}+{ }^{4} J_{\mathrm{HP}}\right|=$ $\left.16.3 \mathrm{~Hz},{ }^{3} J_{\mathrm{HH}}=5.6 \mathrm{~Hz}, 2 \mathrm{H}, \mathrm{NCHCHP}\right), 7.20(\mathrm{~m}, 2 \mathrm{H}$, meta- $\left.\mathrm{C}_{6} \mathrm{H}_{5}\right), 6.97\left(\mathrm{t},{ }^{3} J_{\mathrm{HH}}=7.2 \mathrm{~Hz}, 1 \mathrm{H}\right.$, para $\left.-\mathrm{C}_{6} \mathrm{H}_{5}\right)$, 4.27 (ABXX'B' A', $N=\left|{ }^{2} J_{\mathrm{HP}}+{ }^{4} J_{\mathrm{HP}}\right|=3.7 \mathrm{~Hz},{ }^{3} J_{\mathrm{HH}}=$ $5.5 \mathrm{~Hz}, 2 \mathrm{H}, \mathrm{NCHCHP}), 1.14\left(\mathrm{~A}_{9} \mathrm{XX}^{\prime} \mathrm{A}_{9}^{\prime}, N=\mid{ }^{3} J_{\mathrm{HP}}+\right.$ $\left.{ }^{5} J_{\mathrm{HP}} \mid=7.0 \mathrm{~Hz}, 18 \mathrm{H}, \mathrm{P}\left(\mathrm{C}\left(\mathrm{CH}_{3}\right)_{3}\right)\right), 1.12\left(\mathrm{~A}_{9} \mathrm{XX}^{\prime} \mathrm{A}_{9}^{\prime}, N\right.$ $\left.=\left|{ }^{3} J_{\mathrm{HP}}+{ }^{5} J_{\mathrm{HP}}\right|=7.0 \mathrm{~Hz}, 18 \mathrm{H}, \mathrm{P}\left(\mathrm{C}\left(\mathrm{CH}_{3}\right)_{3}\right)\right)$, -46.52(t, $\left.{ }^{2} J_{\mathrm{HP}}=12.6 \mathrm{~Hz}, 1 \mathrm{H}, \operatorname{Ir} H\right)$.

${ }^{13} C\left\{{ }^{1} H\right\}-N M R: \quad\left(101.25 \mathrm{MHz}, \mathrm{C}_{6} \mathrm{D}_{6}, \mathrm{RT}\right): \delta=163.6\left(\mathrm{AXX}^{\prime} \mathrm{A}\right), N=$ $\left.\left|{ }^{2} J_{\mathrm{CP}}+{ }^{3} J_{\mathrm{CP}}\right|=7.3 \mathrm{~Hz}, \mathrm{NCHCHP}\right), 144.6\left(\mathrm{t},{ }^{3} J_{\mathrm{CP}}\right.$ $=6.1 \mathrm{~Hz}$, ortho- $\left.\mathrm{C}_{6} \mathrm{H}_{5}\right), 128.9\left(\mathrm{t},{ }^{2} J_{\mathrm{CP}}=0.1 \mathrm{~Hz}\right.$, ipso- $\mathrm{C}_{6} \mathrm{H}_{5}$ ), 127.1-126.6 (m, meta- $\mathrm{C}_{6} \mathrm{H}_{5}$ ), 120.5 (s para$\left.\mathrm{C}_{6} \mathrm{H}_{5}\right), 85.8\left(\mathrm{AXX}^{\prime} \mathrm{A}^{\prime}, N=\left|{ }^{1} J_{\mathrm{CP}}+{ }^{3} J_{\mathrm{CP}}\right|=22.6 \mathrm{~Hz}\right.$, $\mathrm{NCHCHP}$ ), 39.7 (AXX'A', $N=\left|{ }^{1} J_{\mathrm{CP}}+{ }^{3} J_{\mathrm{CP}}\right|=$ $\left.12.1 \mathrm{~Hz}, \mathrm{P}\left(\mathrm{C}\left(\mathrm{CH}_{3}\right)_{3}\right)\right), 35.3\left(\mathrm{AXX}^{\prime} \mathrm{A}^{\prime}, N=\mid{ }^{1} J_{\mathrm{CP}}+\right.$ $\left.{ }^{3} J_{\mathrm{CP}} \mid=13.4 \mathrm{~Hz}, \mathrm{P}\left(C\left(\mathrm{CH}_{3}\right)_{3}\right)\right), 29.5\left(\mathrm{~A}_{3} \mathrm{XX}^{\prime} \mathrm{A}_{3}, N=\right.$ $\left.\left|{ }^{2} J_{\mathrm{CP}}+{ }^{4} J_{\mathrm{CP}}\right|=3.0 \mathrm{~Hz}, \mathrm{P}\left(\mathrm{C}\left(C \mathrm{H}_{3}\right)_{3}\right)\right), 29.3\left(\mathrm{~A}_{3} \mathrm{XX}^{\prime} \mathrm{A}_{3}{ }_{3}\right.$, $\left.N=\left|{ }^{2} J_{\mathrm{CP}}+{ }^{4} J_{\mathrm{CP}}\right|=2.7 \mathrm{~Hz}, \mathrm{P}\left(\mathrm{C}\left(\mathrm{CH}_{3}\right)_{3}\right)\right)$.

${ }^{31} P\left\{{ }^{1} H\right\}-N M R: \quad\left(161.93 \mathrm{MHz}, \mathrm{CD}_{2} \mathrm{Cl}_{2}, \mathrm{RT}\right): \delta=61.3(\mathrm{~s})$.

\section{3. $\left[\operatorname{lr}\left\{\mathrm{N}\left(\mathrm{CHCHP}^{t} \mathrm{Bu}_{2}\right)_{2}\right\}\left(\mathrm{CH}_{3}\right) \mathrm{Cl}\right](9)$}

Methyl triflate $(3.7 \mu \mathrm{L} ; 33.7 \mu \mathrm{mol} ; 1.0 \mathrm{eq})$ is added to a solution of $5(35.4 \mathrm{mg} ; 33.3 \mu \mathrm{mol}$; $1.0 \mathrm{eq})$ in toluene $(2 \mathrm{~mL})$ at $-20{ }^{\circ} \mathrm{C}$ and stirred for $5 \mathrm{~min}$. After the color of the solution changes from orange to violet, the solution is filtered off and all volatiles are removed $i$. vac. The residue is dissolved in benzene $(10 \mathrm{~mL})$, filtered off and lyophilized overnight. 9 is obtained as analytically pure violet powder (17.7 mg; $29.5 \mu \mathrm{mol} ; 89 \%$ ). 


\section{Elemental Analysis}

$\mathrm{C}_{21} \mathrm{H}_{43} \mathrm{ClNP}_{2} \mathrm{Ir}(599.19) \quad$ calcd.: C, 42.09; H, 7.23; N, 2.34 .

found: C, 42.43; H, 7.06; N, 1.92 .

\section{Spectroscopic Characterization}

${ }^{1} H-N M R$ :

$\left(300 \mathrm{MHz}, \mathrm{C}_{6} \mathrm{D}_{6}, \mathrm{RT}\right): \delta=6.82\left(\mathrm{ABXX}^{\prime} \mathrm{B}^{\prime} \mathrm{A}^{\prime}, N=\left.\right|^{3} J_{\mathrm{HP}}\right.$ $\left.+{ }^{4} J_{\mathrm{HP}} \mid=17.8 \mathrm{~Hz},{ }^{3} J_{\mathrm{HH}}=5.8 \mathrm{~Hz}, 2 \mathrm{H}, \mathrm{NCHCHP}\right)$,

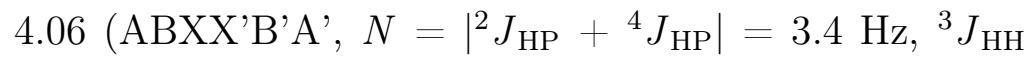
$=6.2 \mathrm{~Hz}, 2 \mathrm{H}, \mathrm{NCHCHP}), 2.08\left(\mathrm{t},{ }^{3} J_{\mathrm{HP}}=5.3 \mathrm{~Hz}, 3 \mathrm{H}\right.$, $\left.\mathrm{Ir}-\mathrm{CH}_{3}\right), 1.32\left(\mathrm{~A}_{9} \mathrm{XX}^{\prime} \mathrm{A}^{\prime}{ }_{9}, N=\left|{ }^{3} J_{\mathrm{HP}}+{ }^{5} J_{\mathrm{HP}}\right|=6.6 \mathrm{~Hz}\right.$, $\left.18 \mathrm{H}, \mathrm{P}\left(\mathrm{C}\left(\mathrm{CH}_{3}\right)_{3}\right)\right), 1.24\left(\mathrm{~A}_{9} \mathrm{XX}^{\prime} \mathrm{A}^{\prime}{ }_{9}, N=\left|{ }^{3} J_{\mathrm{HP}}+{ }^{5} J_{\mathrm{HP}}\right|\right.$ $\left.=6.6 \mathrm{~Hz}, 18 \mathrm{H}, \mathrm{P}\left(\mathrm{C}\left(\mathrm{CH}_{3}\right)_{3}\right)\right),-46.52\left(\mathrm{t},{ }^{2} J_{\mathrm{HP}}=12.6 \mathrm{~Hz}\right.$, $1 \mathrm{H}, \operatorname{Ir} H)$.

${ }^{13} C\left\{{ }^{1} H\right\}-N M R: \quad\left(101.25 \mathrm{MHz}, \mathrm{C}^{1} \mathrm{D}_{6}, \mathrm{RT}\right): \delta=163.3\left(\mathrm{AXX}^{\prime} \mathrm{A}^{\prime}, N=\left.\right|^{2} J_{\mathrm{CP}}\right.$ $+{ }^{3} J_{\mathrm{CP}} \mid=7.2 \mathrm{~Hz}, \mathrm{NCHCHP}$ ), 85.3 (AXX'A', $N=\left.\right|^{1} J_{\mathrm{CP}}$ $\left.+{ }^{3} J_{\mathrm{CP}} \mid=20.4 \mathrm{~Hz}, \mathrm{NCHCHP}\right), 40.4\left(\mathrm{AXX}^{\prime} \mathrm{A}^{\prime}, N=\mid{ }^{1} J_{\mathrm{CP}}\right.$ $\left.+{ }^{3} J_{\mathrm{CP}} \mid=12.1 \mathrm{~Hz}, \mathrm{P}\left(C\left(\mathrm{CH}_{3}\right)_{3}\right)\right), 36.2\left(\mathrm{AXX}^{\prime} \mathrm{A}^{\prime}, N=\right.$ $\left.\left|{ }^{1} J_{\mathrm{CP}}+{ }^{3} J_{\mathrm{CP}}\right|=12.1 \mathrm{~Hz}, \mathrm{P}\left(C\left(\mathrm{CH}_{3}\right)_{3}\right)\right), 30.6\left(\mathrm{~A}_{3} \mathrm{XX}^{\prime} \mathrm{A}_{3}^{\prime}\right.$, $\left.N=\left|{ }^{2} J_{\mathrm{CP}}+{ }^{4} J_{\mathrm{CP}}\right|=2.2 \mathrm{~Hz}, \mathrm{P}\left(\mathrm{C}\left(C \mathrm{H}_{3}\right)_{3}\right)\right), 30.0$ $\left(\mathrm{A}_{3} \mathrm{XX}^{\prime} \mathrm{A}_{3}^{\prime}, N=\left.\right|^{2} J_{\mathrm{CP}}+{ }^{4} J_{\mathrm{CP}} \mid=2.2 \mathrm{~Hz}, \mathrm{P}\left(\mathrm{C}\left(\mathrm{CH}_{3}\right)_{3}\right)\right)$, $-27.1\left(\mathrm{t},{ }^{3} J_{\mathrm{CP}}=4.0 \mathrm{~Hz}, \mathrm{Ir}-\mathrm{CH}_{3}\right)$.

${ }^{31} P\left\{{ }^{1} H\right\}-N M R: \quad\left(161.93 \mathrm{MHz}, \mathrm{CD}_{2} \mathrm{Cl}_{2}, \mathrm{RT}\right): \delta=41.9(\mathrm{~s})$. 


\section{4. $\left[\operatorname{Ir}\left\{\mathrm{N}\left(\mathrm{CHCHP}^{t} \mathrm{Bu}_{2}\right)_{2}\right\}\left(\mathrm{O}_{2}\right)\right](10)$}

\section{Conversion of 5 with oxygen}

A solution of 5 (60.0 mg; $56.4 \mu \mathrm{mol})$ in THF $(20 \mathrm{~mL})$ is degassed by one pump-freezethaw cycle, the reaction vessel is backfilled with oxygen $(1$ bar $)$ and stirred at $-60{ }^{\circ} \mathrm{C}$ for $1 \mathrm{~h}$. All volatiles are removed $i$. vac., the residue is washed with pentanes $(2 \times 5 \mathrm{~mL})$ and extracted with THF $(3 \times 5 \mathrm{~mL})$. After filtration, the THF solution is layered with pentanes $(45 \mathrm{~mL})$ and the product is crystallized at $-32{ }^{\circ} \mathrm{C}$. Red crystals of $\mathbf{1 0}$ are collected by filtration, washed with pentanes and dried $i$. vac. (26.3 mg; $45.2 \mu \mathrm{mol} ; 80 \%$ ).

\section{Elemental Analysis}

$\mathrm{C}_{20} \mathrm{H}_{40} \mathrm{NO}_{2} \mathrm{P}_{2} \operatorname{Ir}(580.71) \quad$ calcd.: C, 41.37; H, 6.94; N, 2.41 .

found: $\mathrm{C}, 41.06 ; \mathrm{H}, 6.67 ; \mathrm{N}, 2.23$.

\section{Conversion of 24 with air}

A solution of $24(100.0 \mathrm{mg} ; 176.9 \mu \mathrm{mol})$ in THF $(20 \mathrm{~mL})$ is exposed for $1 \mathrm{~min}$ to air and further stirred for $3 \mathrm{~h}$ in a closed vessel. All volatiles are removed i. vac. and the residue is extracted with pentanes $(4 \times 5 \mathrm{~mL})$. After evaporation of the to dryness, the crude product is dissolved in toluene $(10 \mathrm{~mL})$ and crystallized at $-35{ }^{\circ} \mathrm{C}$. The crystals are collected by filtration, washed with cold pentanes $(2 \times 5 \mathrm{~mL})$ and lyophilized out of benzene (10 mL). 10 is obtained as red powder (36.0 mg; $62.0 \mu \mathrm{mol} ; 35 \%$ ).

\section{Elemental Analysis}

$\mathrm{C}_{20} \mathrm{H}_{40} \mathrm{NO}_{2} \mathrm{P}_{2} \mathrm{Ir}(580.71) \quad$ calcd.: C, 41.37; H, 6.94; N, 2.41.

found: C, 41.04; H, 6.95; N, 2.26 . 


\section{Spectroscopic Characterization}

${ }^{1} H-N M R:$

(300 MHz, $d_{8}$ THF, RT): $\delta=6.77$ (ABXX'B'A', $N=$ $\left.\left|{ }^{3} J_{\mathrm{HP}}+{ }^{4} J_{\mathrm{HP}}\right|=17.9 \mathrm{~Hz},{ }^{3} J_{\mathrm{HH}}=6.0 \mathrm{~Hz}, 2 \mathrm{H}, \mathrm{NCHCHP}\right)$, $4.76\left(\mathrm{ABXX}^{\prime} \mathrm{B}^{\prime} \mathrm{A}^{\prime}, N=\left.\right|^{2} J_{\mathrm{HP}}+{ }^{4} J_{\mathrm{HP}} \mid=4.4 \mathrm{~Hz},{ }^{3} J_{\mathrm{HH}}=\right.$ $6.0 \mathrm{~Hz}, 2 \mathrm{H}, \mathrm{NCHCHP}), 1.39\left(\mathrm{~A}_{18} \mathrm{XX}^{\prime} \mathrm{A}^{\prime}{ }_{18}, N=\left.\right|^{3} J_{\mathrm{HP}}+\right.$ $\left.{ }^{5} J_{\mathrm{HP}}=6.2 \mathrm{~Hz}, 36 \mathrm{H}, \mathrm{P}\left(\mathrm{C}\left(\mathrm{CH}_{3}\right)_{3}\right)_{2}\right)$.

${ }^{13} C\left\{{ }^{1} H\right\}-N M R$ :

$\left(75.5 \mathrm{MHz}, d_{8^{-}}\right.$THF, RT): $\delta=166.9$ (AXX'A', $N=$ $\left|{ }^{2} J_{\mathrm{CP}}+{ }^{3} J_{\mathrm{CP}}\right|=7.3 \mathrm{~Hz}, \mathrm{NCHCHP}$ ), 98.7 (AXX'A', $N=$ $\left.\left|{ }^{1} J_{\mathrm{CP}}+{ }^{3} J_{\mathrm{CP}}\right|=18.7 \mathrm{~Hz}, \mathrm{NCHCHP}\right), 36.2\left(\mathrm{~A}_{2} \mathrm{XX}^{\prime} \mathrm{A}_{2}^{\prime}\right.$, $\left.N=\left|{ }^{1} J_{\mathrm{CP}}+{ }^{3} J_{\mathrm{CP}}\right|=11.5 \mathrm{~Hz}, \mathrm{P}\left(C\left(\mathrm{CH}_{3}\right)_{3}\right)_{2}\right), 29.8$ $\left(\mathrm{A}_{6} \mathrm{XX}^{\prime} \mathrm{A}_{6}^{\prime}, N=\left.\right|^{2} J_{\mathrm{CP}}+{ }^{4} J_{\mathrm{CP}} \mid=3.0 \mathrm{~Hz}, \mathrm{P}\left(\mathrm{C}\left(C \mathrm{H}_{3}\right)_{3}\right)_{2}\right)$.

${ }^{31} P\left\{{ }^{1} H\right\}-N M R: \quad\left(121 \mathrm{MHz}, d_{8^{-}}\right.$THF, $\left.\mathrm{RT}\right): \delta=43.8(\mathrm{~s})$.

IR:

(Nujol-mull $/ \mathrm{KBr}) \nu=910$ vs $(\nu(\mathrm{OO}))$. 


\section{Iridium nitrido complexes}

\section{1. $\left[\operatorname{Ir}\left\{\mathrm{N}\left(\mathrm{CHCHP}^{t} \mathrm{Bu}_{2}\right)_{2}\right\}\left(\mathrm{N}_{3}\right)\right](11)$}

A solid mixture of 3 (100.1 mg; $0.17 \mathrm{mmol} ; 1 \mathrm{eq})$, [( $\left.\left(\mathrm{PPh}_{3}\right)_{2} \mathrm{~N}\right] \mathrm{N}_{3}(99.5 \mathrm{mg}, 0.17 \mathrm{mmol}$; $1 \mathrm{eq})$ and $\mathrm{NaN}_{3}(100.3 \mathrm{mg}, 1.54 \mathrm{mmol} ; 9 \mathrm{eq})$ is dissolved in acetone $(5 \mathrm{~mL})$ and stirred for $0.5 \mathrm{~h}$ (preparation and work-up were performed at $0{ }^{\circ} \mathrm{C}$ ). After removal of the solvent, the residue is extracted with benzene $(3 \times 4 \mathrm{~mL})$ and the solvent is removed $i$. vac. The crude product is extracted with pentanes $(5 \times 4 \mathrm{~mL})$, evaporated to dryness and the residue is lyophilized out of benzene (6 mL). 11 (84.5 mg; $0.14 \mathrm{mmol}$; 83\%) is obtained as a blue powder.

Elemental Analysis

$\mathrm{C}_{20} \mathrm{H}_{40} \mathrm{IrN}_{4} \mathrm{P}_{2}$ (580.72) calcd.: C, 40.66; H, 6.83; N, 9.48.

found: $\mathrm{C}, 40.76 ; \mathrm{H}, 6.94 ; \mathrm{N}, 9.55$.

\section{Spectroscopic Characterization}

${ }^{1} H-N M R: \quad\left(400 \mathrm{MHz}, \mathrm{C}_{6} \mathrm{D}_{6}, \mathrm{RT}\right): \delta=9.3\left(\mathrm{br}, \mathrm{P}\left(\mathrm{C}\left(\mathrm{CH}_{3}\right)_{3}\right)_{2}\right),-5.6$

(br, $\mathrm{CH}$ ), -135.9 (br, $\mathrm{CH})$.

IR: $\quad($ Nujol-mull $/ \mathrm{KBr}) \nu=2047$ vs $\left(\nu\left(\mathrm{N}_{3}\right)\right)$.

\section{Magnetic Properties}

Evans method

$$
\mu_{\text {eff }}=2.3 \mu_{\mathrm{B}}
$$




\section{2. ${ }^{15 / 14} \mathrm{~N}^{14} \mathrm{~N}_{2-}\left[\operatorname{Ir}\left\{\mathrm{N}\left(\mathrm{CHCHP}{ }^{t} \mathrm{Bu}_{2}\right)_{2}\right\}\left(\mathrm{N}_{3}\right)\right]$ $\left({ }^{15 / 14} \mathrm{~N}-11\right)$}

The ${ }^{15} \mathrm{~N}$ isotopic enriched azido complex ${ }^{\mathbf{1 5} / \mathbf{1 4}} \mathbf{N}-\mathbf{1 1}$ is prepared by stirring $\mathbf{1 1}$ (50.2 $\mathrm{mg}$; $85 \mu \mathrm{mol} ; 1 \mathrm{eq})$ and $\left[\left(\mathrm{PPh}_{3}\right)_{2} \mathrm{~N}\right]^{15} \mathrm{~N}^{14}{ }_{2}(98.8 \mathrm{mg}, 169.9 \mathrm{mmol} ; 2 \mathrm{eq})$ in acetone $(5 \mathrm{~mL})$ for $0.5 \mathrm{~h}$ at $0{ }^{\circ} \mathrm{C}$. After removal of the solvent $i$. vac, the residue is extracted with benzene $(2 \times 5 \mathrm{~mL})$, dried $i$. vac., and extracted with pentanes $(4 \times 7 \mathrm{~mL})$. The solvent is removed $i$. vac. and the residue is lyophilized out of benzene $(5 \mathrm{~mL}) .{ }^{15} / \mathbf{1 4} \mathrm{N}-11$ (43.4 mg; $73.3 \mu \mathrm{mol} ; 86 \%$ ) is obtained as a blue powder. According to the reaction stoichiometry, the product was $33 \%$ enriched with ${ }^{15} \mathrm{~N}$ isotope in the $\alpha$ position of the azido ligand.

\section{3. $\left[\operatorname{Ir}\left\{\mathrm{N}\left(\mathrm{CHCHP}^{t} \mathrm{Bu}_{2}\right)_{2}\right\}(\mathrm{N}) \mathrm{PF}_{6}(12)\right.$}

A mixture of 11 (50.0 mg; $84.6 \mu \mathrm{mol} ; 1 \mathrm{eq})$ and $\left[\mathrm{Fe}\left(\mathrm{C}_{5} \mathrm{H}_{5}\right)_{2}\right] \mathrm{PF}_{6}(28.0 \mathrm{mg} ; 84.6 \mu \mathrm{mol}$; $1 \mathrm{eq})$ is stirred in DCM $(7 \mathrm{~mL})$ for 15 minutes at room temperature. After removal of the solvent, the residue is washed with benzene $(3 \times 5 \mathrm{~mL})$, and extracted with DCM $(3 \times 3 \mathrm{~mL})$. The solution is layered with pentanes $(20 \mathrm{~mL})$ and stored at $-18 \mathrm{C}$. The solid is filtered off, washed with pentanes $(2 \times 4 \mathrm{~mL})$ and dried $i$. vac. 12 (49.1 mg; $69.4 \mu \mathrm{mol} ; 82 \%)$ is obtained as dark blue crystals.

\section{Elemental Analysis}

$\mathrm{C}_{20} \mathrm{H}_{40} \mathrm{~F}_{6} \mathrm{IrN}_{2} \mathrm{P}_{3}(707.67) \quad$ calcd.: C, 33.94; H, 5.70; N, 3.96.

found: $\mathrm{C}, 34.26 ; \mathrm{H}, 5.83 ; \mathrm{N}, 3.71$. 


\section{Spectroscopic Characterization}

\begin{tabular}{|c|c|}
\hline${ }^{1} H-N M R:$ & $\begin{array}{l}\left(400 \mathrm{MHz}, \mathrm{CD}_{2} \mathrm{Cl}_{2}, \mathrm{RT}\right): \delta=7.22\left(\mathrm{ABXX}^{\prime}{ }^{\prime} \mathrm{A}^{\prime}, N=\right. \\
\left.\left|{ }^{3} J_{\mathrm{HP}}+{ }^{4} J_{\mathrm{HP}}\right|=18.9 \mathrm{~Hz},{ }^{3} J_{\mathrm{HH}}=6.3 \mathrm{~Hz}, 2 \mathrm{H}, \mathrm{NC} H \mathrm{CHP}\right), \\
5.41\left(\mathrm{ABXX}{ }^{\prime} \mathrm{B}^{\prime}, N=\left|{ }^{2} J_{\mathrm{HP}}+{ }^{4} J_{\mathrm{HP}}\right|=6.8 \mathrm{~Hz},{ }^{3} J_{\mathrm{HH}}=\right. \\
6.3 \mathrm{~Hz}, 2 \mathrm{H}, \mathrm{NCHCHP}), 1.63\left(\mathrm{~A}_{18} \mathrm{XX}^{\prime} \mathrm{A}^{\prime}{ }_{18}, N=\left.\right|^{2} J_{\mathrm{HP}}+\right. \\
\left.{ }^{4} J_{\mathrm{HP}} \mid=8.4 \mathrm{~Hz}, 36 \mathrm{H}, \mathrm{P}\left(\mathrm{C}\left(\mathrm{CH}_{3}\right)_{3}\right)_{2}\right) .\end{array}$ \\
\hline${ }^{13} C\left\{{ }^{1} H\right\}-N M R$ : & $\begin{array}{l}\left(101.25 \mathrm{MHz}, \mathrm{CD}_{2} \mathrm{Cl}_{2}, \mathrm{RT}\right): \delta=165.5\left(\mathrm{AXX}^{\prime} \mathrm{A}^{\prime}, N=\right. \\
\left.\left|{ }^{2} J_{\mathrm{CP}}+{ }^{3} J_{\mathrm{CP}}\right|=5.4 \mathrm{~Hz}, \mathrm{~N} C \mathrm{HCHP}\right), 98.8\left(\mathrm{AXX}^{\prime} \mathrm{A}^{\prime}, N=\right. \\
\left.\left|{ }^{1} J_{\mathrm{CP}}+{ }^{3} J_{\mathrm{CP}}\right|=25.3 \mathrm{~Hz}, \mathrm{NCH} C \mathrm{HP}\right), 40.9\left(\mathrm{~A}_{2} \mathrm{XX}^{\prime} \mathrm{A}_{2}^{\prime},\right. \\
\left.N=\left|{ }^{1} J_{\mathrm{CP}}+{ }^{3} J_{\mathrm{CP}}\right|=11.7 \mathrm{~Hz}, \mathrm{P}\left(C\left(\mathrm{CH}_{3}\right)_{3}\right)_{2}\right), 29.9 \\
\left(\mathrm{~A}_{6} \mathrm{XX}^{\prime} \mathrm{A}_{6}^{\prime}, N=\left|{ }^{3} J_{\mathrm{CP}}+{ }^{5} J_{\mathrm{CP}}\right|=1.9 \mathrm{~Hz}, \mathrm{P}\left(\mathrm{C}\left(C \mathrm{H}_{3}\right)_{3}\right)_{2}\right) .\end{array}$ \\
\hline${ }^{15} N-N M R:$ & $\left(40.5 \mathrm{MHz}, \mathrm{CD}_{2} \mathrm{Cl}_{2}, \mathrm{RT}\right): \delta=916.1(\mathrm{~s}, \mathrm{Ir}-N)$ \\
\hline${ }^{31} P\left\{{ }^{1} H\right\}-N M R:$ & $\begin{array}{l}\left(161.93 \mathrm{MHz}, \mathrm{CD}_{2} \mathrm{Cl}_{2}, \mathrm{RT}\right): \delta=107.7\left(\mathrm{~s}, P\left(\mathrm{C}\left(\mathrm{CH}_{3}\right)_{3}\right)_{2}\right), \\
\left.-145 \text { (hept, }{ }^{1} J_{\mathrm{PF}}=710.5 \mathrm{~Hz}, P \mathrm{~F}_{6}\right) .\end{array}$ \\
\hline IR: & $($ Nujol-mull $/ \mathrm{KBr}) \nu=999 \mathrm{~m}\left(\nu\left({ }^{14} \mathrm{~N}\right)\right), 972\left(\nu\left({ }^{15} \mathrm{~N}\right)\right.$. \\
\hline
\end{tabular}

\section{4. $\left[\operatorname{Ir}\left\{\mathrm{N}\left(\mathrm{CHCHP}{ }^{t} \mathrm{Bu}_{2}\right)_{2}\right\}(\mathrm{N})\right](13)$}

\section{Reduction route}

Freshly degassed $d_{8}$-THF is trap-to-trap transferred to 12 (5.0 mg; $\left.7.01 \mu \mathrm{mol} ; 1.0 \mathrm{eq}\right)$ and $\left[\mathrm{Co}\left(\mathrm{C}_{5} \mathrm{Me}_{5}\right)_{2}\right](2.3 \mathrm{mg} ; 7.01 \mu \mathrm{mol} ; 1.0 \mathrm{eq})$ in a $J$-Young NMR tube at $-196{ }^{\circ} \mathrm{C}$. The solution is warmed to $-60{ }^{\circ} \mathrm{C}$ and shaken for $5 \mathrm{~min} .{ }^{1} \mathrm{H}$ NMR spectroscopic characterization at $-40{ }^{\circ} \mathrm{C}$ reveals the same signals for $\mathbf{1 3}$ as observed on the photolysis route (vide infra). 


\section{Photolysis route}

Freshly degassed $d_{8}$-THF is trap-to-trap transferred to $\mathbf{1 1}(6.0 \mathrm{mg} ; 10.15 \mu \mathrm{mol})$ in a J-Young quartz tube. The solution is irradiated with a strong UV lamp (270-600 nm spectral range) at $-80{ }^{\circ} \mathrm{C}$ for $15 \mathrm{~min} .{ }^{1} \mathrm{H}$ NMR spectroscopy at $-40{ }^{\circ} \mathrm{C}$ confirms almost full conversion (ca. 95\%) of $\mathbf{1 1}$ and formation of one main product (about 65\%), which was assigned to complex 13, besides smaller amounts of coupling products 14 (about $20 \%$ ) and $\mathbf{7}$ (about 10\%). For kinetic examination, the same amount of $\mathbf{1 1}$ (with $3.2 \mathrm{mg}$ naphthalene as internal standard) was photolyzed under the same conditions and heated to room temperature. The decay of $\mathbf{1 3}$ was monitored by ${ }^{1} \mathrm{H}$ NMR spectroscopy over $50 \mathrm{~min}$.

\section{EPR spectroscopic characterization}

A solution of $\mathbf{1 1}$ in toluene is frozen to a green-transparent glass in liquid $\mathrm{N}_{2}$. This glass is subsequently cooled to $20 \mathrm{~K}$ inside the EPR cavity. Photolysis of azido complex $\mathbf{1 1}$ with UV light to form the nitrido complex $\mathbf{1 3}$ is performed inside the EPR cavity at $20 \mathrm{~K}$, using a strong UV lamp (270-600 nm spectral range) and glass-fiber technology.

\section{IR spectroscopic characterization}

A KBr pellet of $\mathbf{1 1}$ is irradiated with a strong UV lamp (270-600 nm spectral range) at room temperature for $30 \mathrm{~min}$.

\section{Spectroscopic Characterization}
${ }^{1} H$-NMR: $\quad\left(400 \mathrm{MHz}, d_{8}\right.$-THF, $\left.-40{ }^{\circ} \mathrm{C}\right): \delta=7.77$ (br, $36 \mathrm{H}$, $\left.\mathrm{P}\left(\mathrm{C}\left(\mathrm{CH}_{3}\right)_{2}\right)_{2}\right),-13.4$ (br, 2H, $\left.\mathrm{CH}\right),-63.2(\mathrm{br}, 2 \mathrm{H}, \mathrm{CH})$.
${ }^{1} H$-NMR: $\quad\left(400 \mathrm{MHz}, \quad d_{8}\right.$-THF, RT): $\delta=6.55$ (br, $36 \mathrm{H}$, $\left.\mathrm{P}\left(\mathrm{C}\left(\mathrm{CH}_{3}\right)_{2}\right)_{2}\right), \quad-9.5$ (br, 2H, $\left.\mathrm{CH}\right),-49.2$ (br, 2H, $\mathrm{CH})$.

IR:

$($ Nujol-mull $/ \mathrm{KBr}) \nu=901\left(\nu\left({ }^{14} \mathrm{~N}\right)\right), 874\left(\nu\left({ }^{15} \mathrm{~N}\right)\right.$. 


\section{5. $\left[\operatorname{Ir}\left\{\mathrm{N}\left(\mathrm{CHCHP}{ }^{t} \mathrm{Bu}_{2}\right)_{2}\right\}\left(\mathrm{N}_{2}\right)\right](7)$}

$11(9.8 \mathrm{mg} ; 16.6 \mu \mathrm{mol})$ is heated in benzene $(0.5 \mathrm{~mL})$ at $60{ }^{\circ} \mathrm{C}$ for $0.5 \mathrm{~h}$ in a sealed tube. The solvent is removed $i$. vac., and the product is isolated by column chromatography ( $5 \mathrm{~cm}$ silanized silica gel; $0.5 \mathrm{~cm}$ diameter; pentanes). After lyophilization in benzene $(1 \mathrm{~mL}), 7$ is obtained as a bright yellow powder (Yield: $4.9 \mathrm{mg} ; 8.4 \mu \mathrm{mol} ; 51 \%$ ).

\section{Elemental Analysis}

$\mathrm{C}_{20} \mathrm{H}_{40} \mathrm{IrN}_{3} \mathrm{P}_{2}(576.72) \quad$ calcd: C, 41.65; H, 6.99; N, 7.29.

found: $\mathrm{C}, 42.52 ; \mathrm{H}, 6.57 ; \mathrm{N}, 6.57$.

\section{Spectroscopic Characterization}

\begin{tabular}{|c|c|}
\hline${ }^{H} H-N M R$ & $\begin{array}{l}\left(400.13 \mathrm{MHz}, \mathrm{C}_{6} \mathrm{D}_{6}, \mathrm{RT}\right): \delta=6.85\left(\mathrm{ABXX}{ }^{\prime}{ }^{\prime} \mathrm{A}^{\prime}, N=\right. \\
\left.\left|{ }^{3} J_{\mathrm{HP}}+{ }^{4} J_{\mathrm{HP}}\right|=18.9 \mathrm{~Hz},{ }^{3} J_{\mathrm{HH}}=5.6 \mathrm{~Hz}, 2 \mathrm{H}, \mathrm{NC} H \mathrm{CHP}\right), \\
4.27\left(\mathrm{ABXX} \mathrm{B}^{\prime} \mathrm{A}^{\prime}, N=\left.\right|^{2} J_{\mathrm{HP}}+{ }^{4} J_{\mathrm{HP}} \mid=4.4 \mathrm{~Hz},{ }^{3} J_{\mathrm{HH}}=\right. \\
5.0 \mathrm{~Hz}, 2 \mathrm{H}, \mathrm{NCHC} H \mathrm{P}), 1.38\left(\mathrm{~A}_{18} \mathrm{XX}{ }^{\prime} \mathrm{A}^{\prime}{ }_{18}, N={ }^{3} J_{\mathrm{HP}}+\right. \\
\left.{ }^{5} J_{\mathrm{HP}}=6.7 \mathrm{~Hz}, 36 \mathrm{H}, \mathrm{P}\left(\mathrm{C}\left(\mathrm{CH}_{3}\right)_{3}\right)_{2}\right) .\end{array}$ \\
\hline${ }^{13} C\left\{{ }^{1} H\right\}-N M R:$ & $\begin{array}{l}\left(101.25 \mathrm{MHz}, \mathrm{C}_{6} \mathrm{D}_{6}, \mathrm{RT}\right): \delta=164.8\left(\mathrm{AXX}^{\prime} \mathrm{A}^{\prime}, N=\right. \\
\left.\left|{ }^{2} J_{\mathrm{CP}}+{ }^{3} J_{\mathrm{CP}}\right|=9.6 \mathrm{~Hz}, \mathrm{~N} C \mathrm{HCHP}\right), 85.2\left(\mathrm{AXX}^{\prime} \mathrm{A}^{\prime}, N=\right. \\
\left.\left|{ }^{1} J_{\mathrm{CP}}+{ }^{3} J_{\mathrm{CP}}\right|=21.7 \mathrm{~Hz}, \mathrm{NCH} C \mathrm{HP}\right), 37.3\left(\mathrm{~A}_{2} \mathrm{XX}^{\prime} \mathrm{A}_{2},\right. \\
\left.N=\left|{ }^{1} J_{\mathrm{CP}}+{ }^{3} J_{\mathrm{CP}}\right|=12.7 \mathrm{~Hz}, \mathrm{P}\left(C\left(\mathrm{CH}_{3}\right)_{3}\right)_{2}\right), 30.1 \\
\left(\mathrm{~A}_{6} \mathrm{XX}^{\prime} \mathrm{A}_{6}^{\prime}, N=\left|{ }^{\prime} J_{\mathrm{CP}}+{ }^{5} J_{\mathrm{CP}}\right|=2.9 \mathrm{~Hz}, \mathrm{P}\left(\mathrm{C}\left(C \mathrm{H}_{3}\right)_{3}\right)_{2}\right) .\end{array}$ \\
\hline${ }^{15} N\left\{{ }^{1} H\right\}-N M R:$ & $\begin{array}{l}\left(40.5 \mathrm{MHz}, d_{8^{-}} \mathrm{THF}, \mathrm{RT}\right): \delta=364.8(\mathrm{~s}, 1 \mathrm{~N}, \operatorname{Ir}-\mathrm{N} N), 331.3 \\
(\mathrm{~s}, 1 \mathrm{~N}, . \operatorname{Ir}-N \mathrm{~N}) .\end{array}$ \\
\hline${ }^{31} P\left\{{ }^{1} H\right\}-N M R$ & $\left(101.25 \mathrm{MHz}, d_{8^{-}} \mathrm{THF}, \mathrm{RT}\right): \delta=70.0(\mathrm{~s})$ \\
\hline$I R$ & (Nujol-mull/KBr): $\nu=2077$ vs $\left(\nu\left(\mathrm{N}_{2}\right)\right)$ \\
\hline
\end{tabular}




\section{6. $\left[\operatorname{Ir}\left\{\mathrm{N}\left(\mathrm{CHCHP}{ }^{t} \mathrm{Bu}_{2}\right)_{2}\right\}\right]_{2}\left(\mathrm{~N}_{2}\right)(14)$}

Freshly degassed THF $(0.5 \mathrm{~mL})$ was trap-to-trap transferred to a mixture of $\mathbf{1 2}(17.0 \mathrm{mg}$; $24.0 \mu \mathrm{mol})$ and $\mathrm{KC}_{8}(3.6 \mathrm{mg} ; 26.4 \mu \mathrm{mol})$ in a $J$-Young NMR tube and backfilled with Argon. After thawing the solvent, the mixture was warmed to ambient temperate and shaken for 5 minutes to give a brown solution. According to ${ }^{1} \mathrm{H}$ and ${ }^{31} \mathrm{P}$ NMR spectroscopy, the solution contained $\mathbf{1 4}$ and minor amounts of $\mathbf{7}$ (ca. 4\%). Attempted isolation of 14 was unsuccessful owing to decomposition during work-up. A sample enriched in ${ }^{15} \mathrm{~N}$ was obtained using ${ }^{\mathbf{1 5} / \mathbf{1 4}} \mathrm{N}-\mathbf{1 2}$ as starting material.

\section{Spectroscopic Characterization}

${ }^{1} H-N M R$ :

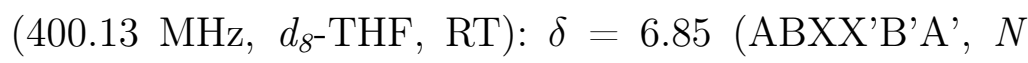
$=\left|{ }^{3} J_{\mathrm{HP}}+{ }^{4} J_{\mathrm{HP}}\right|=18.2 \mathrm{~Hz},{ }^{3} J_{\mathrm{HH}}=5.7 \mathrm{~Hz}, 4 \mathrm{H}$, $\mathrm{NCHCHP}$ ), 4.26 (ABXX'B'A', $N=\left|{ }^{2} J_{\mathrm{HP}}+{ }^{4} J_{\mathrm{HP}}\right|=$ $\left.3.0 \mathrm{~Hz},{ }^{3} J_{\mathrm{HH}}=5.5 \mathrm{~Hz}, 4 \mathrm{H}, \mathrm{NCHCHP}\right), 1.39\left(\mathrm{~A}_{18} \mathrm{XX}^{\prime} \mathrm{A}^{\prime}{ }_{18}\right.$, $\left.N=\left|{ }^{3} J_{\mathrm{HP}}+{ }^{5} J_{\mathrm{HP}}\right|=6.5 \mathrm{~Hz}, 72 \mathrm{H}, \mathrm{P}\left(\mathrm{C}\left(\mathrm{CH}_{3}\right)_{3}\right)_{2}\right)$.

${ }^{13} C\left\{{ }^{1} H\right\}-N M R: \quad\left(101.25 \mathrm{MHz}, d_{8}\right.$-THF, RT) $: \delta=164.5\left(\right.$ AXX'A'$^{\prime}, N=$ $\left|{ }^{2} J_{\mathrm{CP}}+{ }^{3} J_{\mathrm{CP}}\right|=9.3 \mathrm{~Hz}, \mathrm{NCHCHP}$ ), 85.8 (AXX'A', $N=$ $\left.\left|{ }^{1} J_{\mathrm{CP}}+{ }^{3} J_{\mathrm{CP}}\right|=21.7 \mathrm{~Hz}, \mathrm{NCHCHP}\right), 37.0\left(\mathrm{~A}_{2} \mathrm{XX}^{\prime} \mathrm{A}_{2}^{\prime}\right.$, $\left.N=\left|{ }^{1} J_{\mathrm{CP}}+{ }^{3} J_{\mathrm{CP}}\right|=12.0 \mathrm{~Hz}, \mathrm{P}\left(C\left(\mathrm{CH}_{3}\right)_{3}\right)_{2}\right), 31.3$ $\left(\mathrm{A}_{6} \mathrm{XX}^{\prime} \mathrm{A}_{6}^{\prime}, N=\left.\right|^{2} J_{\mathrm{CP}}+{ }^{4} J_{\mathrm{CP}} \mid=3.2 \mathrm{~Hz}, \mathrm{P}\left(\mathrm{C}\left(C \mathrm{H}_{3}\right)_{3}\right)_{2}\right)$.

${ }^{15} N\left\{{ }^{1} H\right\}-N M R: \quad\left(40.5 \mathrm{MHz}, d_{8}\right.$ THF, RT) $: \delta=320.1(\mathrm{~s}, 1 \mathrm{~N})$.

${ }^{31} P\left\{{ }^{1} H\right\}-N M R: \quad\left(101.25 \mathrm{MHz}, d_{8}\right.$ THF, $\left.\mathrm{RT}\right): \delta=65.8(\mathrm{~s}, 2 \mathrm{P})$.

\section{Mass Spectrometric Characterization}

$\operatorname{HRMS}(\mathrm{m} / \mathrm{z})[\mathrm{M}+\mathrm{H}]^{+} \quad$ calcd.: 1125.4643 .

$\mathrm{C}_{40} \mathrm{H}_{80} \mathrm{Ir}_{2} \mathrm{~N}_{4} \mathrm{P}_{4}$

found.: 1125.4681 . 


\subsection{Coupling of 13 in the presence of 1,4-cyclohexadiene}

$d_{8}$-THF $(0.45 \mathrm{~mL})$ was trap-to-trap transferred from Na/K to $11(5.0 \mathrm{mg} ; 8.5 \mu \mathrm{mol}$; 1.0 eq) in a $J$-Young NMR tube. After thawing, the solution was irradiated for $15 \mathrm{~min}$ at $-60{ }^{\circ} \mathrm{C}$ and frozen at $77 \mathrm{~K} .1,4$-Cyclohexadiene $\left(50 \mu \mathrm{L} ; 0.73 \mathrm{M}\right.$ in $d_{8}$-THF; $\left.4.3 \mathrm{eq}\right)$ was added by trap-to-trap transfer and the solution was shaken at room temperature. Monitoring of the reaction by ${ }^{1} \mathrm{H}$ and ${ }^{31} \mathrm{P}$ NMR spectroscopy revealed conversion to $\mathbf{1 4}$ and 7 and no reaction of 1,4-cyclohexadiene. 


\section{Rhodium nitrido complexes}

\section{1. $\left[\mathrm{Rh}\left\{\mathrm{N}\left(\mathrm{CHCHP}{ }^{t} \mathrm{Bu}_{2}\right)_{2}\right\} \mathrm{Cl}\right](15)$}

A solution of 1 (390.5 mg; $1.08 \mathrm{mmol} ; 1 \mathrm{eq})$ in THF $(5 \mathrm{~mL})$ is added to a suspension of $\left[\mathrm{Rh}\left(\mathrm{PPh}_{3}\right)_{3} \mathrm{Cl}\right](1.00 \mathrm{~g} ; 1.08 \mathrm{mmol} ; 1 \mathrm{eq})$ in THF $(15 \mathrm{~mL})$ and stirred at room temperature. After $20 \mathrm{~min}$, a solution of para-benzoquinone (291.8 mg; $2.70 \mathrm{mmol} ; 2.5 \mathrm{eq}$ ) in THF $(10 \mathrm{~mL})$ is added drop wise and the mixture is stirred for additional $16 \mathrm{~h}$. The suspension is extracted with THF $(3 \times 10 \mathrm{~mL})$ and the solvent is removed $i$. vac. The residue is extracted with benzene $(7 \times 5 \mathrm{~mL})$, the solvent is evaporated to dryness and the crude product is extracted with pentanes $(20 \times 10 \mathrm{~mL})$. After removal of the solvent $i$. vac., the remaining solid is lyophilized out of benzene $(20 \mathrm{~mL})$ and heated $i$. vac. for $4 \mathrm{~h}$ at $60{ }^{\circ} \mathrm{C}$. The residue is extracted with pentanes $(10 \times 10 \mathrm{~mL})$, concentrated i. vac. and crystallized for $72 \mathrm{~h}$ at $-32{ }^{\circ} \mathrm{C}$. The solid is filtered off, washed with cold pentanes $(2 \times 5 \mathrm{~mL})$, dissolved in benzene $(20 \mathrm{~mL})$ and lyophilized. 15 (256.0. $\mathrm{mg}$; $517.3 \mu \mathrm{mol} ; 48 \%)$ is obtained as fine green powder.

\section{Elemental Analysis}

$\mathrm{C}_{20} \mathrm{H}_{40} \mathrm{ClRhNP}_{2}$ calcd: C, 49.29; H, 8.33; N, 2.77; P, 12.25 . $\times 0.15 \mathrm{C}_{5} \mathrm{H}_{14}(494.84)$ found: $\mathrm{C}, 49.73 ; \mathrm{H}, 8.20 ; \mathrm{N}, 2.61 ; \mathrm{P}, 12.72$.

For further purification, 15 (50.0 mg; $101.0 \mu \mathrm{mol} ; 1 \mathrm{eq})$ is sublimed $\left(120{ }^{\circ} \mathrm{C}, 30 \mathrm{mTorr}\right.$, $12 \mathrm{~h}$ ) and recovered as dark green micro-crystalline solid (27.4 mg; $55.4 \mu \mathrm{mol} ; 55 \%$ ).

\section{Elemental Analysis}

$\mathrm{C}_{20} \mathrm{H}_{40} \mathrm{ClRhNP}_{2}$ (494.84) calcd: C, 48.54; H, 8.15; N, 2.83.

found: C, 48.62; H, 7.95; N, 2.77 . 


\title{
Spectroscopic Characterization
}

\author{
${ }^{1} H-N M R: \quad\left(250 \mathrm{MHz}, \mathrm{C}_{6} \mathrm{D}_{6}, \mathrm{RT}\right): \delta=10.45\left(\mathrm{br}, 36 \mathrm{H}, \mathrm{P}\left(\mathrm{C}\left(\mathrm{CH}_{3}\right)_{3}\right)_{2}\right)$, \\ -6.77 (br, 2H, CH), -138.17 (br, $2 \mathrm{H}, \mathrm{CH}$ ).
}

\section{Magnetic Properties}

Evans method

$$
\mu_{\text {eff }}=1.7 \mu_{\mathrm{B}}
$$

\section{2. $\left[\operatorname{Rh}\left\{\mathrm{N}\left(\mathrm{CHCHP}{ }^{t} \mathrm{Bu}_{2}\right)_{2}\right\}\left(\mathrm{N}_{3}\right)\right](16)$}

A mixture of 15 (100.0 mg; $202.1 \mu \mathrm{mol} ; 1 \mathrm{eq})$, [( $\left(\mathrm{PPh}_{3}\right)_{2} \mathrm{~N}^{\mathrm{N}} \mathrm{N}_{3}(116.0 \mathrm{mg}, 202.1 \mu \mathrm{mol}$; $1 \mathrm{eq})$ and $\mathrm{NaN}_{3}(118.2 \mathrm{mg}, 1.82 \mathrm{mmol} ; 9 \mathrm{eq})$ is stirred in acetone $(10 \mathrm{~mL})$ for $1 \mathrm{~h}$ at $0{ }^{\circ} \mathrm{C}$ in the dark. After removal of the solvent $i$. vac., the residue is extracted with benzene $(4 \times 7 \mathrm{~mL})$, evaporated to dryness and the remaining solid is extracted with pentanes $(6 \times 10 \mathrm{~mL})$. The solvent is removed $i$. vac., the crude product is dissolved in benzene (15 mL) and lyophilized. 16 (90.5 mg; $180.5 \mu \mathrm{mol} ; 89 \%$ ) is obtained as green powder.

\section{Elemental Analysis}

$\mathrm{C}_{20} \mathrm{H}_{40} \mathrm{RhN}_{4} \mathrm{P}_{2}(501.41) \quad$ calcd.: C, 47.91; H, 8.04; N, 11.17.

found: $\mathrm{C}, 48.07 ; \mathrm{H}, 7.89 ; \mathrm{N}, 10.85$.

\section{Spectroscopic Characterization}

${ }^{1} H-N M R: \quad\left(300 \mathrm{MHz}, \mathrm{C}_{6} \mathrm{D}_{6}, \mathrm{RT}\right): \delta=12.22(\mathrm{br}, \mathrm{CH}), 9.21$ (br, $\left.\mathrm{P}\left(\mathrm{C}\left(\mathrm{CH}_{3}\right)_{3}\right)_{2}\right),-163.5($ br, $\mathrm{CH})$.

$I R:$

(Nujol-mull/KBr) $\nu=2040$ vs $\left(\nu\left(\mathrm{N}_{3}\right)\right)$. 


\section{3. ${ }^{15 / 14} \mathrm{~N}^{14} \mathrm{~N}_{2-}\left[\mathrm{Rh}\left\{\mathrm{N}\left(\mathrm{CHCHP}{ }^{t} \mathrm{Bu}_{2}\right)_{2}\right\}\left(\mathrm{N}_{3}\right)\right]$ $\left({ }^{15 / 14} \mathrm{~N}-16\right)$}

For the synthesis of ${ }^{15} \mathrm{~N}$ labeled 16, a mixture of $\mathbf{1 6}$ (50.0 mg; $\left.99.76 \mu \mathrm{mol} ; 1 \mathrm{eq}\right)$ and $[\mathrm{PPN}]^{15} \mathrm{~N}^{14} \mathrm{~N}_{2}(1159.8 \mathrm{mg} ; 199.53 \mu \mathrm{mol} ; 2.0 \mathrm{eq})$ is stirred in acetone $(10 \mathrm{~mL})$ for $1 \mathrm{~h}$ at $0{ }^{\circ} \mathrm{C}$ in the dark. After removal of the solvent $i$. vac., the residue is extracted with benzene $(3 \times 5 \mathrm{~mL})$, evaporated to dryness, and extracted with pentanes $(5 \times 5 \mathrm{~mL})$. ${ }^{15 / 14} \mathrm{~N}-16$ is obtained as green powder (46.0 mg; $91.78 \mu \mathrm{mol} ; 92 \%$ ). According to the reaction stoichiometry, the product was $33 \%$ enriched with ${ }^{15} \mathrm{~N}$ in the $\alpha$ position of the azido ligand.

\section{4. $\left[\mathrm{Rh}\left\{\mathrm{N}\left(\mathrm{CHCHP}^{t} \mathrm{Bu}_{2}\right)_{2}\right\}(\mathrm{N})\right](17)$}

\section{EPR spectroscopic characterization}

A solution of $\mathbf{1 6}$ in toluene is frozen to a green-transparent glass in liquid $\mathrm{N}_{2}$. This glass is subsequently cooled to $20 \mathrm{~K}$ inside the EPR cavity. Photolysis of azido complex 16 with UV light to from the nitrido complex $\mathbf{1 7}$ is performed inside the EPR cavity at $20 \mathrm{~K}$, using a strong UV lamp (310-600 nm spectral range) and glass-fiber technology.

\section{IR spectroscopic characterization}

A KBr pellet of $\mathbf{1 6}$ is irradiated with a Xenon short-arc lamp for 10 seconds, turned around and irradiated for additional 10 seconds at room temperature. An IR spectrum is measured instantaneously after the photolysis.

\section{Spectroscopic Characterization}

IR:

$$
(\text { Nujol-mull } / \mathrm{KBr}) \nu=874\left(\nu\left({ }^{14} \mathrm{~N}\right)\right), 848\left(\nu\left({ }^{15} \mathrm{~N}\right) .\right.
$$




\section{5. $\left[\mathrm{Rh}\left\{\mathrm{N}\left(\mathrm{CHCHP}^{t} \mathrm{Bu}_{2}\right)_{2}\right\}\left(\mathrm{N}_{2}\right)\right](18)$}

A solution of $16(30.0 \mathrm{mg} ; 59.8 \mu \mathrm{mol})$ in toluene $(0.5 \mathrm{~mL})$ is irradiated for $55 \mathrm{~min}$ at $-60{ }^{\circ} \mathrm{C}$. The solvent is removed $i$. vac. and the crude product is purified by column chromatography (silanized silica gel; pentanes). The solvent is removed $i$. vac. and the residue is lyophilized in benzene $(2 \mathrm{~mL}) . \mathbf{1 8}$ is obtained as bright yellow powder (yield: $11.4 \mathrm{mg} ; 23.7 \mu \mathrm{mol} ; 32 \%)$.

\section{Elemental Analysis}

$\mathrm{C}_{20} \mathrm{H}_{40} \mathrm{~N}_{3} \mathrm{P}_{2} \mathrm{Rh}(487.41) \quad$ calcd.: C, $49.28 ; \mathrm{H}, 8.27 ; \mathrm{N}, 8.62$;

found.: C, 49.30; H, 8.20; N, 8.05;

\section{Spectroscopic Characterization}

${ }^{1} H-N M R:$

$\left(300 \mathrm{MHz}, \mathrm{C}_{6} \mathrm{D}_{6}, \mathrm{RT}\right): \delta=6.90$ (ABMXM'B'A', $N$ $=\left|{ }^{3} J_{\mathrm{HP}}+{ }^{4} J_{\mathrm{HP}}\right|=20.9 \mathrm{~Hz},{ }^{3} J_{\mathrm{HH}}=5.5 \mathrm{~Hz},{ }^{3} J_{\mathrm{HRh}}$ $=2.9 \mathrm{~Hz}, 2 \mathrm{H}, \mathrm{NCHCHP}), 4.05$ (ABMXM'B'A', $N=$ $\left|{ }^{2} J_{\mathrm{HP}}+{ }^{4} J_{\mathrm{HP}}\right|=2.4 \mathrm{~Hz},{ }^{3} J_{\mathrm{HH}}=5.5 \mathrm{~Hz},{ }^{4} J_{\mathrm{HRh}}=1.0 \mathrm{~Hz}$, $2 \mathrm{H}, \mathrm{NCHCHP}), 1.37\left(\mathrm{~A}_{18} \mathrm{XX}^{\prime} \mathrm{A}^{\prime}{ }_{18}, N=\left|{ }^{3} J_{\mathrm{HP}}+{ }^{5} J_{\mathrm{HP}}\right|\right.$ $\left.=6.5 \mathrm{~Hz}, 36 \mathrm{H}, \mathrm{P}\left(\mathrm{C}\left(\mathrm{CH}_{3}\right)_{3}\right)_{2}\right)$.

${ }^{13} C\left\{{ }^{1} H\right\}-N M R: \quad\left(75.5 \mathrm{MHz}, \mathrm{C}_{6} \mathrm{D}_{6}, \mathrm{RT}\right): \delta=162.8$ (AMXM'A', $N=$ $\left.\left|{ }^{2} J_{\mathrm{CP}}+{ }^{3} J_{\mathrm{CP}}\right|=11.2 \mathrm{~Hz},{ }^{2} J_{\mathrm{CRh}}=3.6 \mathrm{~Hz}, \mathrm{NCHCHP}\right)$, 85.8 (AMXM'A', $N=\left|{ }^{1} J_{\mathrm{CP}}+{ }^{3} J_{\mathrm{CP}}\right|=17.6 \mathrm{~Hz}$, $\left.{ }^{2} J_{\mathrm{CRh}}=1.4 \mathrm{~Hz}, \mathrm{NCHCHP}\right), 35.8\left(\mathrm{~A}_{2} \mathrm{MXM}^{\prime}{ }^{\prime}{ }_{2}, N=\right.$ $\left.\left|{ }^{1} J_{\mathrm{CP}}+{ }^{3} J_{\mathrm{CP}}\right|=9.4 \mathrm{~Hz},{ }^{2} J_{\mathrm{CRh}}=1.4 \mathrm{~Hz}, \mathrm{P}\left(C\left(\mathrm{CH}_{3}\right)_{3}\right)_{2}\right)$, $29.9\left(\mathrm{~A}_{6} \mathrm{XX}^{\prime} \mathrm{A}_{6}^{\prime}, \quad N=\left|{ }^{2} J_{\mathrm{CP}}+{ }^{4} J_{\mathrm{CP}}\right|=3.7 \mathrm{~Hz}\right.$, $\left.\mathrm{P}\left(\mathrm{C}\left(\mathrm{CH}_{3}\right)_{3}\right)_{2}\right)$. 
${ }^{31} P\left\{{ }^{1} H\right\}-N M R:$

$\left(202.5 \mathrm{MHz}, \mathrm{C}_{6} \mathrm{D}_{6}, \mathrm{RT}\right): \delta=78.2\left(\mathrm{~d},{ }^{1} J_{\mathrm{PRh}}=136.6 \mathrm{~Hz}\right)$.

$I R:$

(Nujol-mull/KBr $) \quad \nu=2120$ vs $\left(\nu\left({ }^{14} \mathrm{~N} \equiv{ }^{14} \mathrm{~N}\right)\right), 2083$ $\left(\nu\left({ }^{15} \mathrm{~N} \equiv{ }^{14} \mathrm{~N}\right)\right)$.

\section{6. $\left[\mathrm{Rh}\left\{\mathrm{N}\left(\mathrm{CHCHP}^{t} \mathrm{Bu}_{2}\right)_{2}\right\}\right]_{2}\left(\mathrm{~N}_{2}\right)$ (19)}

A degassed solution of $\mathbf{1 6}(20.0 \mathrm{mg} ; 39.85 \mu \mathrm{mol})$ in $d_{8}$-toluene $(0.5 \mathrm{~mL})$ under vacuum is irradiated with a Xenon Short-arc lamp $(150 \mathrm{~W})$ for 30 minutes at $-60{ }^{\circ} \mathrm{C}$. The solution is degassed again and irradiated for 15 minutes in three alternating cycles and finally degassed. A mixture of $\mathbf{1 9}$ and $\mathbf{1 8}$ is obtained as an orange solution in 66\% (19) and $34 \%$ (18) yield ( ${ }^{31} \mathrm{P}$ and $\left.{ }^{1} \mathrm{H} \mathrm{NMR}\right)$, respectively, which could not be separated.

\section{Spectroscopic Characterization}

${ }^{1} H-N M R$ :

(300 MHz, $d_{8}$-toluene, RT): $\delta=6.81$ (ABMXM'B'A', $N=\left|{ }^{3} J_{\mathrm{HP}}+{ }^{4} J_{\mathrm{HP}}\right|=20.2 \mathrm{~Hz},{ }^{3} J_{\mathrm{HH}}=5.4 \mathrm{~Hz},{ }^{3} J_{\mathrm{HRh}}$ $=2.9 \mathrm{~Hz}, 4 \mathrm{H}, \mathrm{NCHCHP}), 4.03$ (ABMXM'B'A', $N=$ $\left.\left|{ }^{2} J_{\mathrm{HP}}+{ }^{4} J_{\mathrm{HP}}\right|=2.7 \mathrm{~Hz},{ }^{3} J_{\mathrm{HH}}=5.4 \mathrm{~Hz}, 4 \mathrm{H}, \mathrm{NCHCHP}\right)$, $1.43\left(\mathrm{~A}_{18} \mathrm{XX}^{\prime} \mathrm{A}^{\prime}{ }_{18}, N=\left|{ }^{3} J_{\mathrm{HP}}+{ }^{5} J_{\mathrm{HP}}\right|=6.5 \mathrm{~Hz}, 72 \mathrm{H}\right.$, $\left.\mathrm{P}\left(\mathrm{C}\left(\mathrm{CH}_{3}\right)_{3}\right)_{2}\right)$.

${ }^{13} C\left\{{ }^{1} H\right\}$-NMR: $\quad\left(75.5 \mathrm{MHz}, d_{8}\right.$-toluene, RT): $\delta=162.5$ (AMXM'A', $N=\left|{ }^{2} J_{\mathrm{CP}}+{ }^{3} J_{\mathrm{CP}}\right|=10.7 \mathrm{~Hz},{ }^{2} J_{\mathrm{CRh}}=3.8 \mathrm{~Hz}$, $\mathrm{NCHCHP}$ ), 82.9 (AMXM'A', $N=\left|{ }^{1} J_{\mathrm{CP}}+{ }^{3} J_{\mathrm{CP}}\right|=$ $\left.17.7 \mathrm{~Hz},{ }^{2} J_{\mathrm{CRh}}=1.4 \mathrm{~Hz}, \mathrm{NCHCHP}\right), 35.5\left(\mathrm{~A}_{2} \mathrm{MXM}^{\prime} \mathrm{A}_{2}\right.$, $N=\left|{ }^{1} J_{\mathrm{CP}}+{ }^{3} J_{\mathrm{CP}}\right|=8.6 \mathrm{~Hz},{ }^{2} J_{\mathrm{CRh}}=1.3 \mathrm{~Hz}$, $\left.\mathrm{P}\left(C\left(\mathrm{CH}_{3}\right)_{3}\right)_{2}\right), 30.9\left(\mathrm{~A}_{6} \mathrm{XX}^{\prime} \mathrm{A}_{6}^{\prime}, N=\left|{ }^{2} J_{\mathrm{CP}}+{ }^{4} J_{\mathrm{CP}}\right|=\right.$ $\left.3.8 \mathrm{~Hz}, \mathrm{P}\left(\mathrm{C}\left(\mathrm{CH}_{3}\right)_{3}\right)_{2}\right)$. 
${ }^{15} N\left\{{ }^{1} H\right\}-N M R: \quad\left(50.7 \mathrm{MHz}, d_{8}\right.$-toluene, $\left.\mathrm{RT}\right): \delta=-75.8\left(\mathrm{~d},{ }^{1} J_{\mathrm{NRh}}=\right.$ $26.1 \mathrm{~Hz}, 1 \mathrm{~N})$.

${ }^{31} P\left\{{ }^{1} H\right\}-N M R: \quad\left(202.5 \mathrm{MHz}, \quad d_{8}\right.$-toluene, $\left.\quad \mathrm{RT}\right): \quad \delta=75.1 \quad(\mathrm{~d}$, $\left.{ }^{1} J_{\mathrm{PRh}}=142.1 \mathrm{~Hz}, 2 \mathrm{P}\right)$.

$\mathrm{ESI}^{+}(\mathrm{m} / \mathrm{z})[\mathrm{M}+\mathrm{H}]^{+}$

$\mathrm{C}_{40} \mathrm{H}_{80} \mathrm{Rh}_{2} \mathrm{~N}_{4} \mathrm{P}_{4}$

calcd.: 947.352 .

found.: 947.375 .

$\mathrm{C}_{40} \mathrm{H}_{80} \mathrm{Rh}_{2}{ }^{15} \mathrm{~N}^{14} \mathrm{~N}_{3} \mathrm{P}_{4}$

calcd.: 948.349 .

found.: 948.375 .

\section{Mass Spectrometric Characterization}

\subsection{Photolysis of 16 in the presence of 1,4-cyclohexadiene}

1,4-Cyclohexadiene $(0.9 \mu \mathrm{L} ; 99.7 \mu \mathrm{mol} ; 10 \mathrm{eq})$ is added to a solution of 16 (5.0 mg; $9.97 \mu \mathrm{mol} ; 1.0 \mathrm{eq})$ in $d_{8}$-toluene $(0.5 \mathrm{~mL})$ and irradiated for $15 \mathrm{~min}$ at $-60{ }^{\circ} \mathrm{C}$. Monitoring of the reaction by ${ }^{1} \mathrm{H}$ and ${ }^{31} \mathrm{P}$ NMR spectroscopy revealed 19 and 18 as sole reaction products and no conversion of 1,4-cyclohexadiene. 


\section{Amino and amido complexes of iridium}

\section{1. $\left[\operatorname{Ir}\left\{\mathrm{N}\left(\mathrm{CHCHP}^{t} \mathrm{Bu}_{2}\right)_{2}\right\}\left(\mathrm{NH}_{2}\right)\right](24)$}

$\mathrm{C}_{10} \mathrm{H}_{20} \mathrm{O}_{5}(51.4 \mu \mathrm{L} ; 256.8 \mu \mathrm{mol} ; 1 \mathrm{eq})$ is added to a solution of $\mathbf{3}$ (150 mg; $256.8 \mu \mathrm{mol}$; $1 \mathrm{eq})$ and $\mathrm{NaNH}_{2}(100.2 \mathrm{mg} ; 2.57 \mathrm{mmol} ; 10 \mathrm{eq})$ in THF $(20 \mathrm{~mL})$ and stirred for $2.5 \mathrm{~h}$ at room temperature. After removal of all volatiles $i$. vac., the residue is extracted with benzene $(3 \times 7 \mathrm{~mL})$ and the solvent is removed. After extraction of the crude product with pentanes $(8 \times 5 \mathrm{~mL})$ and filtration over celite, the solution is evaporated to dryness and the product is lyophilized overnight out of benzene $(15 \mathrm{~mL}) \mathbf{2 4}$ is obtained as green powder (130.0 mg; $230.2 \mu \mathrm{mol} ; 90 \%$ ) with residues of not identified crown-ether adducts. For higher purity, a sample of $\mathbf{2 4}$ was sublimated for $16 \mathrm{~h}$ at $75{ }^{\circ} \mathrm{C} i$. vac. $60 \% \mathrm{w}$ could be collected.

\section{Elemental Analysis}

$\mathrm{C}_{20} \mathrm{H}_{42} \mathrm{~N}_{2} \mathrm{P}_{2} \mathrm{Ir}(564.73) \quad$ calcd.: C, 42.54; H, 7.50; N, 4.96; P, 10.97 .

after sublimation found: $\mathrm{C}, 43.27 ; \mathrm{H}, 7.56 ; \mathrm{N}, 4.69$. 


\section{Spectroscopic Characterization}

${ }^{1} H$-NMR: $\quad\left(300 \mathrm{MHz}, \quad \mathrm{C}_{6} \mathrm{D}_{6}, \quad 20 \quad{ }^{\circ} \mathrm{C}\right): \quad \delta=5.96$ (br, $36 \mathrm{H}$, $\left.\mathrm{P}\left(\mathrm{C}\left(\mathrm{CH}_{3}\right)_{3}\right)_{2}\right),-33.08\left(\mathrm{br}, 2 \mathrm{H}, \mathrm{NH}_{2}\right),-53.88$ (br, $\left.2 \mathrm{H}, \mathrm{CH}\right)$, -73.11 (br, 2H, CH).

\section{Magnetic Properties}

Evans method

$$
\mu_{e f f}=1.82 \mu_{\mathrm{B}}
$$

\section{2. $\left[\operatorname{Ir}\left\{\mathrm{N}\left(\mathrm{CHCHP}^{t} \mathrm{Bu}_{2}\right)_{2}\right\}\left(\mathrm{NH}_{2}\right)\right] \mathrm{PF}_{6}(21)$}

A mixture of $24(50.0 \mathrm{mg} ; 88.5 \mu \mathrm{mol} ; 1 \mathrm{eq})$ and $\mathrm{AgPF}_{6}(22.4 \mathrm{mg} ; 88.5 \mu \mathrm{mol} ; 1 \mathrm{eq})$ dissolved in THF $(20 \mathrm{~mL})$ is stirred for $5 \mathrm{~min}$. After removal of the solvent, the residue is washed with benzene $(4 \times 5 \mathrm{~mL})$ and the crude product is extracted with THF $(8 \times 5 \mathrm{~mL})$. The solution is concentrated, layered with pentanes $(40 \mathrm{~mL})$ and crystallized at $-32{ }^{\circ} \mathrm{C}$ for $6 \mathrm{~h}$. The crystals are collected by filtration, washed with pentanes $(4 \times 5 \mathrm{~mL})$ and dried $i$. vac. 21 is obtained as deep purple crystals $(50.3 \mathrm{mg} ; 63.0 \mu \mathrm{mol}$; $71 \%)$.

\section{Elemental Analysis}

$\mathrm{C}_{20} \mathrm{H}_{42} \mathrm{~N}_{2} \mathrm{P}_{3} \mathrm{~F}_{6} \operatorname{Ir}(709.7)$

calcd: C, 33.85; H, 5.97; N, 3.95

found: $\mathrm{C}, 33.99 ; \mathrm{H}, 5.78 ; \mathrm{N}, 3.48$. 


\section{Spectroscopic Characterization}

${ }^{1} H-N M R$ :

(300 MHz, $\left.\mathrm{CD}_{2} \mathrm{Cl}_{2}, \mathrm{RT}\right): \delta=11.35$ (br, 2H, $\left.\operatorname{IrNH} H_{2}\right)$, $6.35\left(\mathrm{ABXX}^{\prime} \mathrm{B}^{\prime} \mathrm{A}^{\prime}, N=\left.\right|^{2} J_{\mathrm{HP}}+{ }^{4} J_{\mathrm{HP}} \mid=4.7 \mathrm{~Hz},{ }^{3} J_{\mathrm{HH}}=\right.$ $6.3 \mathrm{~Hz}, 2 \mathrm{H}, \mathrm{NCHCHP}$ ), 6.11 (ABXX'B'A', $N=\left.\right|^{3} J_{\mathrm{HP}}$ $\left.+{ }^{5} J_{\mathrm{HP}}=17.6 \mathrm{~Hz},{ }^{3} J_{\mathrm{HH}}=6.3 \mathrm{~Hz}, 2 \mathrm{H}, \mathrm{NCHCHP}\right)$, $1.66\left(\mathrm{~A}_{18} \mathrm{XX}^{\prime} \mathrm{A}^{\prime}{ }_{18}, N=\left|{ }^{3} J_{\mathrm{HP}}+{ }^{5} J_{\mathrm{HP}}\right|=7.5 \mathrm{~Hz}, 36 \mathrm{H}\right.$, $\left.\mathrm{P}\left(\mathrm{C}\left(\mathrm{CH}_{3}\right)_{3}\right)_{2}\right)$.

${ }^{13} C-N M R$ :

$\left(75.5 \mathrm{MHz}, \mathrm{CD}_{2} \mathrm{Cl}_{2}, \mathrm{RT}\right): \delta=169.6\left(\mathrm{AXX}^{\prime} \mathrm{A}^{\prime}, \quad N=\right.$ $\left.\left|{ }^{2} J_{\mathrm{CP}}+{ }^{3} J_{\mathrm{CP}}\right|=6.0 \mathrm{~Hz}, \mathrm{NCHCHP}\right), 121.2$ (AXX'A', $N$ $\left.=\left|{ }^{1} J_{\mathrm{CP}}+{ }^{3} J_{\mathrm{CP}}\right|=18.4 \mathrm{~Hz}, \mathrm{NCHCHP}\right), 36.5\left(\mathrm{~A}_{2} \mathrm{XX}^{\prime} \mathrm{A}_{2}^{\prime}\right.$, $\left.N=\left|{ }^{1} J_{\mathrm{CP}}+{ }^{3} J_{\mathrm{CP}}\right|=11.7 \mathrm{~Hz}, \quad \mathrm{PC}\left(\mathrm{CH}_{3}\right)_{3}\right), 31.4$ $\left(\mathrm{A}_{6} \mathrm{XX}^{\prime} \mathrm{A}_{6}^{\prime}, N=\left.\right|^{2} J_{\mathrm{CP}}+{ }^{4} J_{\mathrm{CP}} \mid=2.7 \mathrm{~Hz}, \mathrm{PC}\left(\mathrm{CH}_{3}\right)_{3}\right)$.

${ }^{31} P\left\{{ }^{1} H\right\}-N M R: \quad\left(121.5 \mathrm{MHz}, \mathrm{CD}_{2} \mathrm{Cl}_{2}, \mathrm{RT}\right): \delta=48.2\left(\mathrm{~s}, P\left(\mathrm{C}\left(\mathrm{CH}_{3}\right)_{3}\right)_{2}\right)$, -145.0 (hept, ${ }^{1} J_{\mathrm{PF}}=710.5 \mathrm{~Hz}, \mathrm{PF}_{6}$ ).

\section{3. $\left[\operatorname{Ir}\left\{\mathrm{N}\left(\mathrm{CHCHP}^{t} \mathrm{Bu}_{2}\right)_{2}\right\}\left(\mathrm{NH}_{3}\right)\right]\left[\mathrm{B}\left(\mathrm{C}_{6} \mathrm{H}_{3}\left(\mathrm{CF}_{3}\right)_{2}\right)_{4}\right]$ (20)}

A mixture of $\mathrm{H}\left[\mathrm{B}\left\{\mathrm{C}_{6} \mathrm{H}_{3}\left(\mathrm{CF}_{3}\right)_{2}\right\}_{4}\right](47.0 \mathrm{mg} ; 65.9 \mu \mathrm{mol} ; 1.24 \mathrm{eq})$ and 24 (30.0 mg; $53.1 \mu \mathrm{mol} ; 1.0 \mathrm{eq})$ is dissolved in $d_{8}$ THF $(0.5 \mathrm{~mL})$ and shaken for 5 minutes. The product is precipitated with pentanes $(2 \mathrm{~mL})$, collected by filtration and washed with pentanes (3 x $2 \mathrm{~mL}$ ). 20 is isolated as blue solid (30.9 mg; $21.6 \mu \mathrm{mol} ; 41 \%$ ).

\section{Elemental Analysis}

$\mathrm{C}_{52} \mathrm{H}_{55} \mathrm{BF}_{24} \mathrm{~N}_{2} \mathrm{P}_{2} \mathrm{Ir}$ (1428.96) calcd.: C, 43.71; H, 3.88; N, 1.96.

found: $\mathrm{C}, 43.91 ; \mathrm{H}, 3.82 ; \mathrm{N}, 1.61$. 


\section{Spectroscopic Characterization}

${ }^{1} H-N M R$ :

$\left(400 \mathrm{MHz}, d-8\right.$ - THF, $\left.20{ }^{\circ} \mathrm{C}\right): \delta=38.3(\mathrm{br}, 2 \mathrm{H}, \mathrm{CH})$, 13.5 (br, 36H, $\left.\mathrm{P}\left(\mathrm{CH}_{3}\right)_{3}\right), 7.77$ (s, 8H, o- $\left.\mathrm{C}_{6} \mathrm{H}_{3}\left(\mathrm{CHF}_{3}\right)_{2}\right)$, $7.54\left(\mathrm{~s}, 4 \mathrm{H}, p-\mathrm{C}_{6} \mathrm{H}_{3}\left(\mathrm{CHF}_{3}\right)_{2}\right)$.

Evans method

$$
\mu_{\text {eff }}=1.40 \mu_{\mathrm{B}}
$$

\subsection{Conversion of 13 with TEMPO-H}

\section{Photolysis route}

A solution of 11 ( $5.0 \mathrm{mg} ; 8.5 \mu \mathrm{mol} ; 1.0 \mathrm{eq})$ in $d_{8}$-toluene $(0.4 \mathrm{~mL})$ containing naphthalene $(3.2 \mathrm{mg})$ as internal standard is photolyzed at $213 \mathrm{~K}$ for 10 minutes and characterized by ${ }^{1} \mathrm{H}$ and ${ }^{31} \mathrm{P}$ NMR spectroscopy. A solution of TEMPO-H (13.3 mg; $\left.84.6 \mu \mathrm{mol} ; 10 \mathrm{eq}\right)$ in $d_{8}$-toluene $(0.1 \mathrm{~mL})$ is added and the reaction mixture is allowed to warm to room temperature. Formation of $\mathbf{2 4}(63 \%)$ and $\mathbf{7}(7 \%)$ is revealed by ${ }^{1} \mathrm{H}$ NMR integration. In addition, unconverted $\mathbf{1 1}(30 \%)$ is still present.

\section{Reduction route}

A mixture of $12(10.0 \mathrm{mg} ; 14.1 \mu \mathrm{mol} ; 1.0 \mathrm{eq})$ and $\left[\mathrm{Co}\left(\mathrm{C}_{5} \mathrm{Me}_{5}\right)_{2}\right](4.7 \mathrm{mg} ; 14.1 \mu \mathrm{mol}$; $1.0 \mathrm{eq})$ is dissolved in $d_{8}$-THF $(0.3 \mathrm{~mL})$ at $-35{ }^{\circ} \mathrm{C}$ and shaken for 5 minutes. A solution of TEMPO-H ( $4.8 \mathrm{mg} ; 30.5 \mu \mathrm{mol} ; 2.2 \mathrm{eq})$ in $d_{8}$-THF $(0.2 \mathrm{~mL})$ is added at $-35{ }^{\circ} \mathrm{C}$ and the mixture is allowed to warm to room temperature. ${ }^{1} \mathrm{H}$ NMR spectroscopy reveals a product mixture of $\mathbf{2 4}(76 \%)$ and $\mathbf{1 4}(24 \%)$. 


\section{Stoichiometric conversion}

A solution containing $11(5.0 \mathrm{mg} ; 8.35 \mu \mathrm{mol} ; 1 \mathrm{eq})$ in $d_{8}$-THF $(0.4 \mathrm{~mL})$ is irradiated at $-60{ }^{\circ} \mathrm{C}$ for 15 minutes and characterized with ${ }^{1} \mathrm{H}$ NMR spectroscopy, revealing a mixture of $\mathbf{1 3}(96 \%)$ and minor amounts of $\mathbf{7}$ and $\mathbf{1 4}$ (4\% in total). A solution of TEMPO-H ( $1.3 \mathrm{mg} ; 8.5 \mu \mathrm{mol} ; 1 \mathrm{eq})$ in $d_{8}$-THF $(0.2 \mathrm{~mL})$ is added and shaken for 1 minute. ${ }^{1} \mathrm{H}$ NMR integration revealed a mixture containing $\mathbf{1 3}(8 \%), \mathbf{2 4}(23 \%), \mathbf{7}$ and $\mathbf{1 4}$ (16\% in total) and one yet unidentified set of signals at $\delta=4.27,48.0 \mathrm{ppm}(54 \%)$.

\subsection{Coupling of 13 in the presence of 2,4,6-tri-tert-butylphenol}

A mixture of $12(10.0 \mathrm{mg} ; 14.1 \mu \mathrm{mol} ; 1.0 \mathrm{eq})$ and $\left[\mathrm{Co}\left(\mathrm{C}_{5} \mathrm{Me}_{5}\right)_{2}\right](4.7 \mathrm{mg} ; 14.1 \mu \mathrm{mol}$; $1.0 \mathrm{eq})$ is dissolved in $d_{8}$-THF $(0.3 \mathrm{~mL})$ at $-35{ }^{\circ} \mathrm{C}$ and shaken for 5 minutes. A solution of 2,4,6-tri-tert-butylphenol $(7.4 \mathrm{mg} ; 28.3 \mu \mathrm{mol} ; 2.0 \mathrm{eq})$ in $d_{8}$-THF $(0.2 \mathrm{~mL})$ is added and shaken. The solution is allowed to warm to room temperature. Selective nitride coupling is indicated by the product mixture consistent of $\mathbf{1 4}(91 \%)$ and minor amounts of $\mathbf{7}(9 \%)$, as observed via ${ }^{1} \mathrm{H}$ and ${ }^{31} \mathrm{P}$ NMR spectroscopy.

\subsection{Conversion of 24 with TEMPO}

A mixture of $\mathbf{2 4}(5.0 \mathrm{mg} ; 8.9 \mu \mathrm{mol} ; 1.0 \mathrm{eq})$ and TEMPO (2.8 mg; $17.7 \mu \mathrm{mol} ; 2.0 \mathrm{eq})$ is dissolved in $\mathrm{C}_{6} \mathrm{D}_{6}(0.5 \mathrm{~mL})$ and shaken for 5 minutes. No reaction is observed by ${ }^{1} \mathrm{H}$ NMR spectroscopy.

\subsection{Reaction of 24 with tri-tert-butylphenoxyl radical}

$d_{8}$-Toluene $(0.5 \mathrm{~mL})$ is trap-to-trap transferred to a mixture of $\mathbf{2 4}(5.0 \mathrm{mg} ; 8.8 \mu \mathrm{mol}$; $1.0 \mathrm{eq}$ ) and 2,4,6-tri-tert-butylphenoxyl radical (4.6 mg; $17.7 \mu \mathrm{mol} ; 2.0 \mathrm{eq})$. The reaction mixture is shaken at $-60{ }^{\circ} \mathrm{C}$ for 5 minutes and allowed to warm to room temperature. Full conversion of the starting material was revealed by ${ }^{1} \mathrm{H}$ NMR spectroscopy. After $1 \mathrm{~h}$ at room temperature, a mixture of $\mathbf{1 4}(73 \%)$ and $\mathbf{7}(13 \%)$ is obtained. 


\subsection{Conversion of 21 with $\mathrm{K}\left[\mathrm{N}\left(\mathrm{SiMe}_{3}\right)_{2}\right]$}

$d_{8}$-THF $(0.5 \mathrm{~mL})$ is stirred over $\mathrm{Na} / \mathrm{K}$-alloy for $0.5 \mathrm{~h}$ and trap-to-trap transferred to a mixture of 21 (10.0 mg; $14.1 \mu \mathrm{mol} ; 1.0 \mathrm{eq}), \mathrm{K}\left[\mathrm{N}\left(\mathrm{SiMe}_{32}\right]\right.$ (2.8 mg; $\left.14.1 \mu \mathrm{mol} ; 1.0 \mathrm{eq}\right)$ and naphthalene $(4.3 \mathrm{mg})$ as internal standard. The reaction is monitored by ${ }^{1} \mathrm{H}$ NMR spectroscopy and is kept in the dark over the course of the experiment. A product mixture consistent of $\mathbf{2 4}(66 \%)$ and $\mathbf{1 4}(33 \%)$ is obtained. 


\section{Chalcogenonitrosyl complexes of iridium}

\section{1. $\left[\operatorname{Ir}\left\{\mathrm{N}\left(\mathrm{CHCHP}^{t} \mathrm{Bu}_{2}\right)_{2}\right\}(\mathrm{NO})\right] \mathrm{PF}_{6}(25)$}

12 (10.0 mg; $14.1 \mu \mathrm{mol} ; 1 \mathrm{eq})$ and trimethylamine- $N$-oxide (1.1 mg; $14.1 \mu \mathrm{mol} ; 1 \mathrm{eq})$ are stirred in DCM $(5 \mathrm{~mL})$ at room temperature. After $16 \mathrm{~h}$, the solution is filtered off, layered with pentanes $(10 \mathrm{~mL})$ and crystallized at $-18{ }^{\circ} \mathrm{C}$. The solid is filtered off, washed with pentanes $(3 \times 2 \mathrm{~mL})$ and dried $i$. vac. $25(5.8 \mathrm{mg} ; 8.0 \mu \mathrm{mol} ; 58 \%)$ is obtained as purple crystalline solid.

Elemental Analysis

$\mathrm{C}_{20} \mathrm{H}_{40} \mathrm{~F}_{6} \mathrm{IrN}_{2} \mathrm{OP}_{3}(724.19) \quad$ calcd: $\mathrm{C}, 33.19 ; \mathrm{H}, 5.57 ; \mathrm{N}, 3.87$.

found: C, 33.47; H, 5.31; N, 3.73.

\section{Spectroscopic Characterization}

${ }^{1} H-N M R: \quad\left(400 \mathrm{MHz}, \mathrm{CD}_{2} \mathrm{Cl}_{2}, \mathrm{RT}\right): \delta=7.04\left(\mathrm{ABXX}{ }^{\prime} \mathrm{B}^{\prime} \mathrm{A} ', N=\right.$ $\left.\left|{ }^{3} J_{\mathrm{HP}}+{ }^{4} J_{\mathrm{HP}}\right|=20.8 \mathrm{~Hz},{ }^{3} J_{\mathrm{HH}}=5.9 \mathrm{~Hz}, 2 \mathrm{H}, \mathrm{NCHCHP}\right)$, $5.18\left(\mathrm{ABXX}^{\prime} \mathrm{B}^{\prime} \mathrm{A}, \quad N=\left|{ }^{2} J_{\mathrm{HP}}+{ }^{4} J_{\mathrm{HP}}\right|=11.6 \mathrm{~Hz},{ }^{3} J_{\mathrm{HH}}\right.$ $=5.8 \mathrm{~Hz}, 2 \mathrm{H}, \mathrm{NCHCHP}), 1.53\left(\mathrm{~A}_{18} \mathrm{XX}^{\prime} \mathrm{A}^{\prime}{ }_{18}, N=\left.\right|^{3} J_{\mathrm{HP}}\right.$ $\left.+{ }^{5} J_{\mathrm{HP}} \mid=8.0 \mathrm{~Hz}, 36 \mathrm{H}, \mathrm{P}\left(\mathrm{C}\left(\mathrm{CH}_{3}\right)_{3}\right)_{2}\right)$. 


$\begin{array}{ll}{ }^{13} C\left\{{ }^{1} H\right\}-N M R: & \left(101.25 \mathrm{MHz}^{\mathrm{CD}} \mathrm{Cl}_{2}, \mathrm{RT}\right): \delta=166.2\left(\mathrm{AXX}^{\prime} \mathrm{A}^{\prime}, N=\right. \\ & \left.\left.\right|^{2} J_{\mathrm{CP}}+{ }^{3} J_{\mathrm{CP}} \mid=7.0 \mathrm{~Hz}, \mathrm{~N} C \mathrm{HCHP}\right), 93.1\left(\mathrm{AXX}^{\prime} \mathrm{A}^{\prime}, N=\right. \\ & \left|{ }^{1} J_{\mathrm{CP}}+{ }^{3} J_{\mathrm{CP}}\right|=24.2 \mathrm{~Hz}, \mathrm{NCHCHP}, 39.9\left(\mathrm{~A}_{2} \mathrm{XX}^{\prime} \mathrm{A}_{2},\right. \\ & \left.N=\left|{ }^{1} J_{\mathrm{CP}}+{ }^{3} J_{\mathrm{CP}}\right|=12.5 \mathrm{~Hz}, \mathrm{P}\left(C\left(\mathrm{CH}_{3}\right)_{3}\right)_{2}\right), 29.8 \\ & \left(\mathrm{~A}_{6} \mathrm{XX} \mathrm{A}^{\prime}{ }_{6}, N=\left|{ }^{3} J_{\mathrm{CP}}+{ }^{5} J_{\mathrm{CP}}\right|=2.2 \mathrm{~Hz}, \mathrm{P}\left(\mathrm{C}\left(C \mathrm{H}_{3}\right)_{3}\right)_{2}\right) . \\ & \left(161.93 \mathrm{MHz}, \mathrm{CD}_{2} \mathrm{Cl}_{2}, \mathrm{RT}\right): \delta=95.9\left(\mathrm{~s}, P\left(\mathrm{C}\left(\mathrm{CH}_{3}\right)_{3}\right)_{2}\right), \\ & -145\left(\text { hept },{ }^{1} J_{\mathrm{PF}}=710.5 \mathrm{~Hz}, P \mathrm{~F}_{6}\right) . \\ { }^{31} P\left\{{ }^{1} H\right\}-N M R: \quad & (\text { Nujol-mull } / \mathrm{KBr}) \nu=1812 \mathrm{vs}(\nu(\mathrm{NO})) . \\ I R: & \end{array}$

\section{2. $\left[\operatorname{lr}\left\{\mathrm{N}\left(\mathrm{CHCHP}{ }^{t} \mathrm{Bu}_{2}\right)_{2}\right\}(\mathrm{NS})\right] \mathrm{PF}_{6}(26)$}

A mixture of 12 (60.0 mg; $84.78 \mu \mathrm{mol} ; 1 \mathrm{eq})$ and elemental sulfur $(21.8 \mathrm{mg} ; 84.78 \mu \mathrm{mol}$; $8 \mathrm{eq})$ is stirred in DCM $(5 \mathrm{~mL})$ at room temperature for $16 \mathrm{~h}$. After extraction with DCM $(3 \times 5 \mathrm{~mL})$, the dark-blue solution is concentrated, layered with pentanes $(50 \mathrm{~mL})$ and crystallized at $-20{ }^{\circ} \mathrm{C}$ for $16 \mathrm{~h}$. The solid is filtered off, washed with pentane $(3 \times 5 \mathrm{~mL})$ and dried i. vac. 26 (33.5 mg; $45.3 \mu \mathrm{mol} ; 53 \%$ ) is obtained as a micro-crystalline solid. ${ }^{\mathbf{1 4} / \mathbf{1 5}} \mathrm{N}-\mathbf{2 6}$ enriched in ${ }^{15} \mathrm{NS}$ (ca. $33 \%$ ) is prepared analogously starting from ${ }^{\mathbf{1 5} / \mathbf{1 4}} \mathrm{N}-\mathbf{1 2}$.

\section{Elemental Analysis}

$\mathrm{C}_{20} \mathrm{H}_{40} \mathrm{~F}_{6} \mathrm{IrN}_{2} \mathrm{SP}_{3}(739.74) \quad$ calcd: C, 32.47; H, 5.45; N, 3.79; S, 4.33.

found: C, 33.06; H, 5.49; N, 3.72; S, 4.59.

\section{Spectroscopic Characterization}

${ }^{1} \mathrm{H}$-NMR: $\quad\left(400 \mathrm{MHz}, \mathrm{CD}_{2} \mathrm{Cl}_{2}, \mathrm{RT}\right): \delta=6.70$ (ABXX'B'A', $N$ $=\left|{ }^{3} J_{\mathrm{HP}}+{ }^{4} J_{\mathrm{HP}}\right|=20.1 \mathrm{~Hz},{ }^{3} J_{\mathrm{HH}}=6.3 \mathrm{~Hz}, 2 \mathrm{H}$, $\mathrm{NCHCHP}$ ), 5.16 (ABXX'B'A', $N=\left|{ }^{2} J_{\mathrm{HP}}+{ }^{4} J_{\mathrm{HP}}\right|=$ $\left.5.5 \mathrm{~Hz},{ }^{3} J_{\mathrm{HH}}=6.1 \mathrm{~Hz}, 2 \mathrm{H}, \mathrm{NCHCHP}\right), 1.62\left(\mathrm{~A}_{18} \mathrm{XX}^{\prime} \mathrm{A}^{\prime}{ }_{18}\right.$, $\left.N=\left|{ }^{3} J_{\mathrm{HP}}+{ }^{5} J_{\mathrm{HP}}\right|=7.9 \mathrm{~Hz}, 36 \mathrm{H}, \mathrm{P}\left(\mathrm{C}\left(\mathrm{CH}_{3}\right)_{3}\right)_{2}\right)$. 
${ }^{13} C\left\{{ }^{1} H\right\}-N M R: \quad\left(101.25 \mathrm{MHz}, \mathrm{CD}_{2} \mathrm{Cl}_{2}, \mathrm{RT}\right): \delta=165.3\left(\mathrm{AXX}^{\prime} \mathrm{A}^{\prime}, N=\right.$ $\left.\left|{ }^{2} J_{\mathrm{CP}}+{ }^{3} J_{\mathrm{CP}}\right|=7.1 \mathrm{~Hz}, \mathrm{NCHCHP}\right), 96.8$ (AXX'A', $N=$ $\left.\left|{ }^{1} J_{\mathrm{CP}}+{ }^{3} J_{\mathrm{CP}}\right|=23.6 \mathrm{~Hz}, \mathrm{NCHCHP}\right), 41.9\left(\mathrm{~A}_{2} \mathrm{XX}^{\prime} \mathrm{A}_{2}^{\prime}\right.$, $\left.N=\left|{ }^{1} J_{\mathrm{CP}}+{ }^{3} J_{\mathrm{CP}}\right|=12.0 \mathrm{~Hz}, \mathrm{P}\left(C\left(\mathrm{CH}_{3}\right)_{3}\right)_{2}\right), 30.1$ $\left(\mathrm{A}_{6} \mathrm{XX}^{\prime} \mathrm{A}_{6}^{\prime}, N=\left|{ }^{3} J_{\mathrm{CP}}+{ }^{5} J_{\mathrm{CP}}\right|=2.1 \mathrm{~Hz}, \mathrm{P}\left(\mathrm{C}\left(C \mathrm{H}_{3}\right)_{3}\right)_{2}\right)$.

${ }^{15} N\left\{{ }^{1} H\right\}-N M R: \quad\left(50.7 \mathrm{MHz}, \mathrm{CD}_{2} \mathrm{Cl}_{2}, \mathrm{RT}\right): \delta=35.3(\mathrm{~s}, \mathrm{Ir} N \mathrm{~S})$.

${ }^{31} P\left\{{ }^{1} H\right\}-N M R: \quad\left(161.93 \mathrm{MHz}, \mathrm{CD}_{2} \mathrm{Cl}_{2}, \mathrm{RT}\right): \delta=84.3\left(\mathrm{~s}, P\left(\mathrm{C}_{\left.\left.\left(\mathrm{CH}_{3}\right)_{3}\right)_{2}\right),}\right.\right.$ -145 (hept, ${ }^{1} J_{\mathrm{PF}}=710.5 \mathrm{~Hz}, P \mathrm{~F}_{6}$ ).

$I R:$

(Nujol-mull/KBr $) \nu=1285$ vs $\left(\nu\left({ }^{14} \mathrm{NS}\right)\right), 1249\left(\nu\left({ }^{15} \mathrm{NS}\right)\right.$.

\section{3. $\left[\operatorname{Ir}\left\{\mathrm{N}\left(\mathrm{CHCHP}{ }^{t} \mathrm{Bu}_{2}\right)_{2}\right\}(\mathrm{NSe})\right] \mathrm{PF}_{6}(27)$}

A mixture of 12 (30.0 mg; $42.39 \mu \mathrm{mol} ; 1 \mathrm{eq})$ and gray selenium (33.5 mg; $423.95 \mu \mathrm{mol}$; 10 eq) is stirred in DCM $(15 \mathrm{~mL})$ in a sealed tube at $40{ }^{\circ} \mathrm{C}$ for 3 days. After cooling to room temperature, the solution is filtered of and the residue is extracted with DCM $(2 \times 4 \mathrm{~mL})$ and layered with pentane $(20 \mathrm{~mL})$. After crystallization at $-32{ }^{\circ} \mathrm{C}$ for $16 \mathrm{~h}$, the solid is filtered off, washed with pentane $(2 \times 5 \mathrm{~mL})$ and dried i. vac. 27 (8.8 mg; $11.2 \mu \mathrm{mol} ; 26 \%)$ is obtained as dark-teal crystalline solid.

Elemental Analysis

$\mathrm{C}_{20} \mathrm{H}_{40} \mathrm{~F}_{6} \mathrm{IrN}_{2} \mathrm{SeP}_{3}$ (786.63) calcd.: C, 30.54; H, 5.13; N, 3.56.

found.: C, 30.72; H, 5.05; N, 3.44. 


\section{Spectroscopic Characterization}

${ }^{1} H-N M R$ :

(300 MHz, $\left.\mathrm{CD}_{2} \mathrm{Cl}_{2}, \mathrm{RT}\right): \delta=6.68$ (ABXX'B'A', $N$ $=\left|{ }^{3} J_{\mathrm{HP}}+{ }^{4} J_{\mathrm{HP}}\right|=20.0 \mathrm{~Hz},{ }^{3} J_{\mathrm{HH}}=6.0 \mathrm{~Hz}, 2 \mathrm{H}$, $\mathrm{NCHCHP}$ ), 5.10 (ABXX'B'A', $N=\left|{ }^{2} J_{\mathrm{HP}}+{ }^{4} J_{\mathrm{HP}}\right|=$ $\left.7.9 \mathrm{~Hz},{ }^{3} J_{\mathrm{HH}}=6.0 \mathrm{~Hz}, 2 \mathrm{H}, \mathrm{NCHCHP}\right), 1.64\left(\mathrm{~A}_{18} \mathrm{XX}^{\prime} \mathrm{A}^{\prime}{ }_{18}\right.$, $\left.N=\left|{ }^{3} J_{\mathrm{HP}}+{ }^{5} J_{\mathrm{HP}}\right|=7.9 \mathrm{~Hz}, 36 \mathrm{H}, \mathrm{P}\left(\mathrm{C}\left(\mathrm{CH}_{3}\right)_{3}\right)_{2}\right)$.

${ }^{13} C\left\{{ }^{1} H\right\}-N M R$ :

$\left(75.5 \mathrm{MHz}, \mathrm{CD}_{2} \mathrm{Cl}_{2}, \mathrm{RT}\right): \delta=164.5\left(\mathrm{AXX}^{\prime} \mathrm{A}^{\prime}, \quad N=\right.$ $\left.\left|{ }^{2} J_{\mathrm{CP}}+{ }^{3} J_{\mathrm{CP}}\right|=7.0 \mathrm{~Hz}, \mathrm{NCHCHP}\right), 97.4$ (AXX' A', $N=$ $\left.\left|{ }^{1} J_{\mathrm{CP}}+{ }^{3} J_{\mathrm{CP}}\right|=23.7 \mathrm{~Hz}, \mathrm{NCHCHP}\right), 42.6\left(\mathrm{~A}_{2} \mathrm{XX}^{\prime} \mathrm{A}_{2}^{\prime}\right.$, $\left.N=\left|{ }^{1} J_{\mathrm{CP}}+{ }^{3} J_{\mathrm{CP}}\right|=11.9 \mathrm{~Hz}, \mathrm{P}\left(C\left(\mathrm{CH}_{3}\right)_{3}\right)_{2}\right), 30.2$ $\left(\mathrm{A}_{6} \mathrm{XX}^{\prime} \mathrm{A}_{6}^{\prime}, N=\left|{ }^{3} J_{\mathrm{CP}}+{ }^{5} J_{\mathrm{CP}}\right|=2.0 \mathrm{~Hz}, \mathrm{P}\left(\mathrm{C}\left(C \mathrm{H}_{3}\right)_{3}\right)_{2}\right)$.

${ }^{31} P\left\{{ }^{1} H\right\}-N M R:$

(121.5 MHz, $\left.\mathrm{CD}_{2} \mathrm{Cl}_{2}, \mathrm{RT}\right): \delta=83.4\left(\mathrm{~s}, P\left(\mathrm{C}\left(\mathrm{CH}_{3}\right)_{3}\right)_{2}\right)$, -145 (hept, ${ }^{1} J_{\mathrm{PF}}=710.5 \mathrm{~Hz}, P \mathrm{~F}_{6}$ ).

$I R:$

(Nujol-mull/KBr) $\nu=1134 \mathrm{~m}(\nu(\mathrm{NSe}))$. 



\section{Part V.}

\section{Literature}




\section{Bibliography}

[1] P. Häussinger, P. Leitgeb, B. Schmücker in Ullmann's Encyclopedia of Industrial Chemistry (Ed.: B. Elvers), Weily-VCH, Weinheim, 7th ed., 2011, pp. 351-375.

[2] H.-P. Jia, E. A. Quadrelli, Chem. Soc. Rev. 2014, 43, 547-564.

[3] A. E. Shilov, Russ. Chem. Bull. 2003, 52, 2555-2562.

[4] E. J. Barclay, G. J. Leigh in Ullmann's Encyclopedia of Industrial Chemistry (Ed.: B. Elvers), Weily-VCH, Weinheim, 7th ed., 2011, pp. 377-390.

[5] M. Appl in Ullmann's Encyclopedia of Industrial Chemistry (Ed.: B. Elvers), Wiley-VCH Verlag GmbH \& Co. KGaA, Weinheim, 7th ed., 2011, pp. 139-225.

[6] B. M. Hoffman, D. Lukoyanov, Z.-Y. Yang, D. R. Dean, L. C. Seefeldt, Chem. Rev. 2014, 114, 4041-4062.

[7] M. M. Georgiadis, H. Komiya, P. Chakrabarti, D. Woo, J. J. Kornuc, D. C. Rees, Science 1992, 25\%, 1653-1659.

[8] O. Einsle, F. A. Tezcan, S. L. A. Andrade, B. Schmid, M. Yoshida, J. B. Howard, D. C. Rees, Science 2002, 297, 1696-1700.

[9] K. M. Lancaster, M. Roemelt, P. Ettenhuber, Y. Hu, M. W. Ribbe, F. Neese, U. Bergmann, S. DeBeer, Science 2011, 334, 974-977.

[10] T. Spatzal, M. Aksoyoglu, L. Zhang, S. L. A. Andrade, E. Schleicher, S. Weber, D. C. Rees, O. Einsle, Science 2011, 334, 940.

[11] B. M. Hoffman, D. R. Dean, L. C. Seefeldt, Acc. Chem. Res. 2009, 42, 609-619.

[12] D. V. Yandulov, R. R. Schrock, Science 2003, 301, 76-78. 
[13] D. V. Yandulov, R. R. Schrock, A. L. Rheingold, C. Ceccarelli, W. M. Davis, Inorg. Chem. 2003, 42, 796-813.

[14] D. V. Yandulov, R. R. Schrock, Inorg. Chem. 2005, 44, 1103-1117.

[15] K. Arashiba, Y. Miyake, Y. Nishibayashi, Nature Chem 2011, 3, 120-125.

[16] J. S. Anderson, J. Rittle, J. C. Peters, Nature 2013, 501, 84-87.

[17] M. Appl in Ullmann's Encyclopedia of Industrial Chemistry (Ed.: B. Elvers), Wiley-VCH Verlag GmbH \& Co. KGaA, Weinheim, 7th ed., 2011, pp. 107-137.

[18] G. Ertl, Angew. Chem. Int. Ed. 2008, 47, 3524-3535.

[19] K. Honkala, A. Hellman, I. N. Remediakis, A. Logadottir, A. Carlsson, S. Dahl, C. H. Christensen, J. K. Nørskov, Science 2005, 307, 555-558.

[20] C. E. Laplaza, C. C. Cummins, Science 1995, 268, 861-863.

[21] E. Solari, C. Da Silva, B. Iacono, J. Hesschenbrouck, C. Rizzoli, R. Scopelliti, C. Floriani, Angew. Chem. Int. Ed. 2001, 113, 4025-4027.

[22] T. J. Hebden, R. R. Schrock, M. K. Takase, P. Müller, Chem. Commun. 2012, 48, $1851-1853$.

[23] D. J. Mindiola, K. Meyer, J.-P. F. Cherry, T. A. Baker, C. C. Cummins, Organometallics 2000, 19, 1622-1624.

[24] J. S. Figueroa, N. A. Piro, C. R. Clough, C. C. Cummins, J. Am. Chem. Soc. 2006, 128, 940-950.

[25] H. Kunkely, A. Vogler, Angew. Chem. Int. Ed. 2010, 49, 1591-1593.

[26] J. M. Smith, D. Subedi, Dalton Trans. 2012, 41, 1423-1429.

[27] I. Klopsch, M. Finger, C. Würtele, B. Milde, D. B. Werz, S. Schneider, J. Am. Chem. Soc. 2014, 136, 6881-6883.

[28] J. Schöffel, A. Y. Y. Rogachev, S. DeBeer George, P. Burger, Angew. Chem. Int. Ed. 2009, 48, 4734-4738. 
[29] B. Askevold, J. T. Nieto, S. Tussupbayev, M. Diefenbach, E. Herdtweck, M. C. Holthausen, S. Schneider, Nature Chem. 2011, 3, 532-537.

[30] J. A. Pool, E. Lobkovsky, P. J. Chirik, Nature 2004, 427, 527-530.

[31] M. M. Rodriguez, E. Bill, W. W. Brennessel, P. L. Holland, Science 2011, 334, 780-783.

[32] K. C. MacLeod, P. L. Holland, Nature Chem. 2013, 5, 559-565.

[33] F. Schüth, R. Palkovits, R. Schlögl, D. S. Su, Energy Environ. Sci. 2012, 5, 62786289.

[34] M. D. Fryzuk, Acc. Chem. Res. 2008, 42, 127-133.

[35] J. G. Andino, S. Mazumder, K. Pal, K. G. Caulton, Angew. Chem. Int. Ed. 2013, 52, 4726-32.

[36] J. Berkowitz, G. B. Ellison, D. Gutman, J. Phys. Chem. 1994, 98, 2744-2765.

[37] J. L. Klinkenberg, J. F. Hartwig, Angew. Chem. Int. Ed. 2011, 50, 86-95.

[38] J. L. Roizen, M. E. Harvey, J. Du Bois, Acc. Chem. Res. 2012, 45, 911-922.

[39] L. Degennaro, P. Trinchera, R. Luisi, Chem. Rev. 2014, 114, 7881-7929.

[40] N. V. Rees, R. G. Compton, Energ. Environ. Sci 2011, 4, 1255-1260.

[41] R. Lan, J. T. Irvine, S. Tao, Int. J. Hydrogen Energy 2012, 37, 1482-1494.

[42] J. M. Mayer, Comments Inorg. Chem. 1988, 125-135.

[43] K. G. Caulton, New J. Chem. 1994, 18, 25-41.

[44] K. Dehnicke, J. Strähle, Angew. Chemie Int. Ed. 1981, 20, 413-426.

[45] C. J. Ballhausen, H. B. Gray, Inorg. Chem. 1962, 1, 111-122.

[46] W. A. Nugent, J. M. Mayer, Metal-Ligand Multiple Bonds, Wiley-VCH Verlag GmbH \& Co. KGaA, New York, 1988.

[47] D. E. Wigley, Prog. Inorg. Chem. 1994, 42, 239-482. 
[48] R. Eikey, M. M. Abu-Omar, Coord. Chem. Rev. 2003, 243, 83-124.

[49] J. F. Berry, Comments Inorg. Chem. 2009, 30, 28-66.

[50] J. M. Smith, Prog. Inorg. Chem. 2014, 58, 417-470.

[51] D. L. Dubois, R. Hoffmann, Nouv. J. Chim. 1977, 1, 479-492.

[52] D. C. Ware, H. Taube, Inorg. Chem. 1991, 30, 4605-4610.

[53] J. M. Mayer, Inorg. Chem. 1988, 27, 3899-3903.

[54] C. T. Saouma, J. C. Peters, Coord. Chem. Rev. 2011, 255, 920-937.

[55] W. D. Wagner, K. Nakamoto, J. Am. Chem. Soc. 1988, 110, 4044-4045.

[56] W. Wagner, K. Nakamoto, J. Am. Chem. Soc. 1989, 1590-1598.

[57] K. Meyer, E. Bill, B. Mienert, T. Weyhermuller, K. Wieghardt, J. Am. Chem. Soc. 1999, 121, 4859-4876.

[58] N. Aliaga-Alcalde, S. DeBeer George, B. Mienert, E. Bill, K. Wieghardt, F. Neese, Angew. Chem. Int. Ed. 2005, 44, 2908-2912.

[59] J. F. Berry, E. Bill, E. Bothe, S. D. George, B. Mienert, F. Neese, K. Wieghardt, Science 2006, 312, 1937-1941.

[60] J. M. Mayer, D. L. Thorn, T. H. Tulip, J. Am. Chem. Soc. 1985, 107, 7454-7462.

[61] E. Spaltenstein, R. R. Conry, S. C. Critchlow, J. M. Mayer, J. Am. Chem. Soc. 1989, 111, 8741-8742.

[62] R. S. Hay-Motherwell, G. Wilkinson, B. Hussain-Bates, M. B. Hursthouse, Polyhedron 1993, 12, 2009-2012.

[63] R. S. Hay-Motherwell, G. Wilkinson, B. Hussain-Bates, M. B. Hursthouse, Polyhedron 1991, 10, 1457-1458.

[64] T. A. Betley, J. C. Peters, J. Am. Chem. Soc. 2004, 126, 6252-6254.

[65] M. P. Hendrich, W. Gunderson, R. K. Behan, M. T. Green, M. P. Mehn, T. A. Betley, C. C. Lu, J. C. Peters, Proc. Natl. Acad. Sci. U. S. A. 2006, 103, 1710717112. 
[66] J.-U. Rohde, T. A. Betley, T. a. Jackson, C. T. Saouma, J. C. Peters, L. Que, Inorg. Chem. 2007, 46, 5720-5726.

[67] C. Vogel, F. W. Heinemann, J. Sutter, C. Anthon, K. Meyer, Angew. Chem. Int. Ed. 2008, 47, 2681-2684.

[68] J. J. Scepaniak, M. D. Fulton, R. P. Bontchev, E. N. Duesler, M. L. Kirk, J. M. Smith, J. Am. Chem. Soc. 2008, 130, 10515-10517.

[69] J. J. Scepaniak, C. S. Vogel, M. M. Khusniyarov, F. W. Heinemann, K. Meyer, J. M. Smith, Science 2011, 331, 1049-1052.

[70] J. Torres-Alacan, U. Das, A. C. Filippou, P. Vöhringer, Angew. Chem. Int. Ed. 2013, 52, 12833-12837.

[71] C. Geng, S. Ye, F. Neese, Dalton Trans. 2014, 43, 6079-6086.

[72] R. Hoffman, Angew. Chem. Int. Ed. Engl. 1982, 21, 711-724.

[73] A. Walstrom, M. Pink, X. Yang, J. Tomaszewski, M.-H. Baik, K. G. Caulton, J. Am. Chem. Soc. 2005, 127, 5330-5331.

[74] D. Buschhorn, M. Pink, H. Fan, K. G. Caulton, Inorg. Chem. 2008, 47, 5129-5135.

[75] M. J. Ingleson, M. Pink, H. Fan, K. G. Caulton, J. Am. Chem. Soc. 2008, 130, $4262-4276$.

[76] K. P. O'Halloran, C. Zhao, N. S. Ando, A. J. Schultz, T. F. Koetzle, P. M. B. Piccoli, B. Hedman, K. O. Hodgson, E. Bobyr, M. L. Kirk, S. Knottenbelt, E. C. Depperman, B. Stein, T. M. Anderson, R. Cao, Y. V. Geletii, K. I. Hardcastle, D. G. Musaev, W. a. Neiwert, X. Fang, K. Morokuma, S. Wu, P. Kögerler, C. L. Hill, Inorg. Chem. 2012, 51, 7025-7031.

[77] R. R. W. Marshman, P. A. Shapley, J. Am. Chem. Soc. 1990, 112, 8369-8378.

[78] Y. Gloaguen, C. Rebreyend, M. Lutz, P. Kumar, M. Huber, J. I. van der Vlugt, S. Schneider, B. de Bruin, Angew. Chemie Int. Ed. 2014, 53, 6814-6818.

[79] W. A. Nugent, R. J. Mckinney, V. Kasowski, F. A. Van-Catledge, Inorganica Chim. Acta 1982, 65, 91-93. 
[80] J. D. Lichtenhan, J. W. Ziller, N. M. Doherty, Inorg. Chem. 1992, 31, 2893-2900.

[81] N. C. Mösch-Zanetti, R. R. Schrock, W. M. Davis, K. Wanninger, S. W. Seidel, M. B. O'Donoghue, J. Am. Chem. Soc. 1997, 119, 11037-11048.

[82] W.-H. Leung, J. L. C. Chim, I. D. Williams, W.-T. Wong, Inorg. Chem. 1999, 38, 3000-3005.

[83] W.-H. Leung, E. Y. Y. Chan, T. C. Y. Lai, W.-T. Wong, J. Chem. Soc. Dalt. Trans. 2000, 51-56.

[84] J. J. Curley, E. L. Sceats, C. C. Cummins, J. Am. Chem. Soc. 2006, 128, 1403614037.

[85] J. S. Silvia, C. C. Cummins, J. Am. Chem. Soc. 2009, 131, 446-447.

[86] B. L. Tran, M. Pink, X. Gao, H. Park, D. J. Mindiola, J. Am. Chem. Soc. 2010, 132, 1458-1459.

[87] K. C. MacLeod, P. L. Holland, J. Scepaniak, R. Bontchev, Angew. Chemie Int. Ed. 2011, 50, 6630-6634.

[88] E. L. Sceats, J. S. Figueroa, C. C. Cummins, N. M. Loening, P. Van der Wel, R. G. Griffin, Polyhedron 2004, 23, 2751-2768.

[89] J. Schöffel, N. Šušnjar, S. Nückel, D. Sieh, P. Burger, Eur. J. Inorg. Chem. 2010, 2010, 4911-4915.

[90] D. Sieh, J. Schoffel, P. Burger, Dalt. Trans. 2011, 40, 9512-9524.

[91] C. C. H. Atienza, A. C. Bowman, E. Lobkovsky, P. J. Chirik, J. Am. Chem. Soc. 2010, 132, 16343-16345.

[92] S. Alvarez, J. Cirera, Angew. Chem. Int. Ed. 2006, 45, 3012-3020.

[93] T. J. Crevier, J. M. Mayer, Angew. Chem. Int. Ed. 1998, 37, 1891-1893.

[94] T. J. Crevier, J. M. Mayer, J. Am. Chem. Soc. 1998, 86, 5595-5596.

[95] T. J. Crevier, B. K. Bennett, J. D. Soper, J. A. Bowman, A. Dehestani, D. A. Hrovat, S. Lovell, W. Kaminsky, J. M. Mayer, J. Am. Chem. Soc. 2001, 123, 1059-1071. 
[96] A. Walstrom, M. Pink, H. Fan, J. Tomaszewski, K. G. Caulton, Inorg. Chem. 2007, 46, 7704-7706.

[97] A. Walstrom, H. Fan, M. Pink, K. G. Caulton, Inorganica Chim. Acta 2010, 363, 633-636.

[98] O. Krahe, E. Bill, F. Neese, Angew. Chem. Int. Ed. 2014, 53, 8727-8731.

[99] C. A. Grapperhaus, B. Mienert, E. Bill, T. Weyhermuller, K. Wieghardt, Inorg. Chem. 2000, 39, 5306-5317.

[100] M. Schlangen, J. Neugebauer, M. Reiher, D. Schröder, J. P. López, M. Haryono, F. W. Heinemann, A. Grohmann, H. Schwarz, J. Am. Chem. Soc. 2008, 130, 4285-4294.

[101] J. J. Scepaniak, J. A. Young, R. P. Bontchev, J. M. Smith, Angew. Chem. Int. Ed. 2009, 48, 3158-3160.

[102] C. S. Vogel, F. W. Heinemann, M. M. Khusniyarov, K. Meyer, Inorg. Chim. Acta 2010, 364, 226-237.

[103] C. R. Clough, J. B. Greco, J. S. Figueroa, P. L. Diaconescu, W. M. Davis, C. C. Cummins, J. Am. Chem. Soc. 2004, 126, 7742-7743.

[104] J. P. Cherry, a. R. Johnson, L. M. Baraldo, Y. C. Tsai, C. C. Cummins, S. V. Kryatov, E. V. Rybak-Akimova, K. B. Capps, C. D. Hoff, C. M. Haar, S. P. Nolan, J. Am. Chem. Soc. 2001, 123, 7271-7286.

[105] S. B. Seymore, S. N. Brown, Inorg. Chem. 2002, 41, 462-469.

[106] M. Shaver, M. Fryzuk, Adv. Synth. Catal. 2003, 345, 1061-1076.

[107] R. D. Sanner, J. M. Manriquez, R. E. Marsh, J. E. Bercaw, J. Am. Chem. Soc. 1976, 98, 8351-8357.

[108] C. E. Laplaza, M. J. A. Johnson, J. C. Peters, A. L. Odom, E. Kim, C. C. Cummins, G. N. George, I. J. Pickering, J. Am. Chem. Soc. 1996, 118, 8623-8638.

[109] R. L. Burwell, R. G. Pearson, J. Phys. Chem. 1966, 70, 300-302.

[110] W.-L. Man, W. W. Y. Lam, T.-C. Lau, Acc. Chem. Res. 2014, 47, 427-439. 
[111] A. Allen, C. Senoff, Chem. Commun. 1965, 1964-1965.

[112] J. Chatt, J. Dilworth, R. Richards, Chem. Rev. 1978, 78, 589-625.

[113] H. A. Scheidegger, J. N. Armor, H. Taube, J. Am. Chem. Soc. 1968, 1147, 32633264 .

[114] J. Chatt, J. Fergusson, Chem. Commun. 1968, 5706.

[115] L. L. A. P. Kane-Maguire, P. S. Sheridan, F. Basolo, R. G. Pearson, J. Am. Chem. Soc. 1968, 90, 5295-5296.

[116] O. Ishitani, P. S. White, T. J. Meyer, Inorg. Chem. 1996, 35, 2167-2168.

[117] A. D. Allen, F. Bottomley, R. O. Harris, V. P. Reinsalu, C. V. Senoff, J. Am. Chem. Soc. 1967, 89, 5595-5599.

[118] J. Collman, J. Kang, J. Am. Chem. Soc. 1966, 769, 3459-3460.

[119] D. E. Harrison, H. Taube, J. Am. Chem. Soc. 1967, 89, 5706-5707.

[120] R. A. Bauer, F. Basolo, Inorg. Chem. 1969, 8, 2231-2236.

[121] L. A. P. Kane-Maguire, P. S. Sheridan, F. Basolo, R. G. Pearson, J. Am. Chem. Soc. 1970, 92, 5865-5872.

[122] H. Gafney, J. Reed, F. Basolo, J. Am. Chem. Soc. 1973, 103, 7998-8005.

[123] J. Reed, H. Gafney, F. Basolo, J. Am. Chem. Soc. 1974, 531, 1363-1369.

[124] J. D. Buhr, H. Taube, Inorg. Chem. 1979, 18, 2208-2212.

[125] K. D. Demadis, T. J. Meyer, P. S. White, Inorg. Chem. 1997, 36, 5678-5679.

[126] K. D. Demadis, E. S. El-Samanody, G. M. Coia, T. J. Meyer, J. Am. Chem. Soc. 1999, 121, 535-544.

[127] E. S. El-Samanody, K. D. Demadis, T. J. Meyer, P. S. White, Inorg. Chem. 2001, 40, 3677-86.

[128] R. Magnuson, H. Taube, J. Am. Chem. Soc. 1972, 1147, 6-7. 
[129] C.-M. Che, H.-W. Lam, W.-F. Tong, T.-F. Lai, T.-C. Lau, J. Chem. Soc. Chem. Commun. 1989, 1883-1884.

[130] G. M. Coia, K. D. Demadis, T. J. Meyer, Inorg. Chem. 2000, 39, 2212-2223.

[131] K. D. Demadis, T. J. Meyer, P. S. White, Inorg. Chem. 1997, 36, 5678-5679.

[132] W. L. Man, T. M. Tang, T. W. Wong, T. C. Lau, S. M. Peng, W. T. Wong, J. Am. Chem. Soc. 2004, 126, 478-479.

[133] H. W. Lam, C. M. Che, K. Y. Wong, J. Chem. Soc. Dalt. Trans. 1992, 1411-1416.

[134] J. Buhr, J. Winkler, H. Taube, Inorg. Chem. 1980, 9, 2416-2425.

[135] D. C. Ware, H. Taube, Inorg. Chem. 1991, 30, 4605-4610.

[136] J. Du Bois, C. S. Tomooka, J. Hong, E. M. Carreira, Acc. Chem. Res. 1997, 30, 364-372.

[137] D. Pipes, M. Bakir, S. E. Vitols, D. J. Hodgson, T. J. Meyer, J. Am. Chem. Soc. 1990, 112, 5507-5514.

[138] O. Ishitani, E. Ando, T. J. Meyer, Inorg. Chem. 2003, 42, 1707-1710.

[139] D. Mindiola, C. Cummins, Angew. Chem. Int. Ed. 1998, 1997-1999.

[140] B. Askevold, M. M. Khusniyarov, E. Herdtweck, K. Meyer, S. Schneider, Angew. Chem. Int. Ed. 2010, 49, 7566-7569.

[141] J. Cirera, E. Ruiz, S. Alvarez, Chem. Eur. J. 2006, 12, 3162-3167.

[142] M. F. Lappert, P. P. Power, A. R. Sanger, R. C. Srivastava, Metal and Metalloid Amides Synthesis, Structures, and Physical and Chemical Properties, John Wiley \& Sons, Inc., Chichester, 1980.

[143] B. Cetinkaya, M. F. Lappert, S. Torroni, J. Chem. Soc. Chem. Commun. 1979, 843-844.

[144] M. D. Fryzuk, P. A. MacNeil, J. Am. Chem. Soc. 1981, 103, 3592-3593.

[145] M. D. Fryzuk, P. A. MacNeil, S. J. Rettig, A. S. Secco, J. Trotter, Organometallics 1982, 1, 918-930. 
[146] M. D. Fryzuk, P. A. MacNeil, Organometallics 1982, 1, 1540-1541.

[147] M. Fryzuk, P. MacNeil, Organometallics 1983, 355-356.

[148] M. D. Fryzuk, P. A. MacNeil, S. J. Rettig, Organometallics 1986, 5, 2469-2476.

[149] W. Baratta, E. Herdtweck, P. Rigo, Angew. Chemie Int. Ed. 1999, 38, 1629-1631.

[150] L. A. Watson, O. V. Ozerov, M. Pink, K. G. Caulton, J. Am. Chem. Soc. 2003, 125, 8426-8427.

[151] A. Walstrom, M. Pink, N. P. Tsvetkov, H. Fan, M. Ingleson, K. G. Caulton, J. Am. Chem. Soc. 2005, 127, 16780-16781.

[152] S. Schneider, J. Meiners, B. Askevold, Eur. J. Inorg. Chem. 2012, 2012, 412-429.

[153] O. V. Ozerov, C. Guo, V. A. Papkov, B. M. Foxman, J. Am. Chem. Soc. 2004, 126, 4792-4793.

[154] L. Fan, L. Yang, C. Guo, B. M. Foxman, O. V. Ozerov, Organometallics 2004, 23, 4778-4787.

[155] L. Fan, S. Parkin, O. V. Ozerov, J. Am. Chem. Soc. 2005, 127, 16772-16773.

[156] L. Fan, O. V. Ozerov, Chem. Commun. 2005, 4450-4452.

[157] M. G. Scheibel, Master Thesis, Technical University Munich, 2010.

[158] V. F. Kuznetsov, K. Abdur-Rashid, A. J. Lough, D. G. Gusev, J. Am. Chem. Soc. 2006, 128, 14388-14396.

[159] M. Käss, A. Friedrich, M. Drees, S. Schneider, Angew. Chem. Int. Ed. 2009, 48, 905-907.

[160] A. Friedrich, M. Drees, M. Käss, E. Herdtweck, S. Schneider, Inorg. Chem. 2010, 49, 5482-5494.

[161] A. Friedrich, R. Ghosh, R. Kolb, E. Herdtweck, S. Schneider, Organometallics 2009, 28, 708-718.

[162] J. Meiners, A. Friedrich, E. Herdtweck, S. Schneider, Organometallics 2009, 28, 6331-6338. 
[163] J. Meiners, M. G. Scheibel, M.-H. Lemée-Cailleau, S. A. Mason, M. B. Boeddinghaus, T. F. Fässler, E. Herdtweck, M. M. Khusniyarov, S. Schneider, Angew. Chem. Int. Ed. 2011, 50, 8184-8187.

[164] F. N. Penkert, T. Weyhermüller, E. Bill, P. Hildebrandt, S. Lecomte, K. Wieghardt, J. Am. Chem. Soc. 2000, 122, 9663-9673.

[165] T. Büttner, J. Geier, G. Frison, J. Harmer, C. Calle, A. Schweiger, H. Schönberg, H. Grützmacher, Science 2005, 307, 235-238.

[166] P. Maire, M. Königsmann, A. Sreekanth, J. Harmer, A. Schweiger, H. Grützmacher, J. Am. Chem. Soc. 2006, 128, 6578-6580.

[167] N. Donati, D. Stein, T. Büttner, H. Schönberg, J. Harmer, S. Anadaram, H. Grützmacher, Eur. J. Inorg. Chem. 2008, 2008, 4691-4703.

[168] R. G. Hicks, Angew. Chem. Int. Ed. 2008, 47, 7393-7395.

[169] M. Königsmann, N. Donati, D. Stein, H. Schönberg, J. Harmer, A. Sreekanth, H. Grützmacher, Angew. Chem. Int. Ed. 2007, 46, 3567-3570.

[170] Y. Miyazato, T. Wada, J. Muckerman, E. Fujita, K. Tanaka, Angew. Chemie Int. Ed. 2007, 46, 5728-5730.

[171] D. Adhikari, S. Mossin, F. Basuli, J. C. Huffman, R. K. Szilagyi, K. Meyer, D. J. Mindiola, J. Am. Chem. Soc. 2008, 130, 3676-3682.

[172] C. Tejel, M. A. Ciriano, M. P. del Río, D. G. H. Hetterscheid, N. Tsichlis i Spithas, J. M. M. Smits, B. de Bruin, Chem. Eur. J. 2008, 14, 10932-10936.

[173] K. K. Pandey, Coord. Chem. Rev. 1992, 121, 1-42.

[174] D. G. DeWit, Coord. Chem. Rev. 1996, 147, 209-246.

[175] R. Poli, Chem. Rev. 1996, 96, 2135-2204.

[176] B. De Bruin, D. G. H. Hetterscheid, A. J. J. Koekkoek, H. Grützmacher, Prog. Inorg. Chem. 2008, 55, 247-354.

[177] M. P. García, M. V. Jiménez, L. A. Oro, F. J. Lahoz, P. J. Alonso, Angew. Chem. Int. Ed. English 1992, 31, 1527-1529. 
[178] A. A. Danopoulos, G. Wilkinson, B. Hussain-Bates, M. B. Hursthouse, J. Chem. Soc. Dalt. Trans. 1992, 3165-3170.

[179] H. Zhai, A. Bunn, B. Wayland, Chem. Commun. 2001, 1294-1295.

[180] B. de Bruin, T. P. J. Peters, S. Thewissen, A. N. J. Blok, J. B. M. Wilting, R. de Gelder, J. M. M. Smits, A. W. Gal, Angew. Chem. Int. Ed. 2002, 41, 21352138 .

[181] A. S. Ionkin, W. J. Marshall, Organometallics 2004, 23, 6031-6041.

[182] D. G. H. Hetterscheid, J. Kaiser, E. Reijerse, T. P. J. Peters, S. Thewissen, A. N. J. Blok, J. M. M. Smits, R. de Gelder, B. de Bruin, J. Am. Chem. Soc. 2005, 127, 1895-1905.

[183] N. P. Tsvetkov, M. F. Laird, H. Fan, M. Pink, K. G. Caulton, Chem. Commun. 2009, 4578-4580.

[184] J. Hohenberger, K. Ray, K. Meyer, Nature Commun. 2012, 3, 720-733.

[185] R. K. Thomson, T. Cantat, B. L. Scott, D. E. Morris, E. R. Batista, J. L. Kiplinger, Nature Chem. 2010, 2, 723-729.

[186] A. K. M. Long, R. P. Yu, G. H. Timmer, J. F. Berry, J. Am. Chem. Soc. 2010, 132, 12228-12230.

[187] A. K. M. Long, G. H. Timmer, J. S. Pap, J. L. Snyder, R. P. Yu, J. F. Berry, J. Am. Chem. Soc. 2011, 133, 13138-13150.

[188] M. W. Bishop, J. Chatt, J. R. Dilworth, J. Chem. Soc. Chem. Commun. 1975, $780-781$.

[189] T. J. Crevier, S. Lovell, J. M. Mayer, A. L. Rheingold, I. A. Guzei, J. Am. Chem. Soc. 1998, 120, 6607-6608.

[190] B. L. Tran, R. Thompson, S. Ghosh, X. Gao, C.-H. Chen, M.-H. Baik, D. J. Mindiola, Chem. Commun. 2013, 49, 2768-2770.

[191] G. Ujaque, A. C. Cooper, F. Maseras, O. Eisenstein, K. G. Caulton, J. Am. Chem. Soc. 1998, 120, 361-365. 
[192] M. Gallagher, N. L. Wieder, V. K. Dioumaev, P. J. Carroll, D. H. Berry, Organometallics 2010, 29, 591-603.

[193] M. Kinauer, M. G. Scheibel, J. Abbenseth, F. W. Heinemann, P. Stollberg, C. Würtele, S. Schneider, Dalton Trans. 2014, 43, 4506-4513.

[194] M. Kinauer, Master Thesis, Georg-August University Göttingen, 2013.

[195] S. S. Oster, W. D. Jones, Polyhedron 2004, 23, 2959-2965.

[196] R. C. Schnabel, D. M. Roddick, Organometallics 1996, 15, 3550-3555.

[197] M. A. F. Hernandez-Gruel, J. S. J. Pérez-Torrente, M. A. Ciriano, L. A. Oro, Inorg. Synth. 2004, 34, 127.

[198] R. Meij, D. Stufkens, K. Vrieze, W. Van Gerresheim, C. Stam, J. Organomet. Chem. 1979, 164, 353-370.

[199] M. T. Whited, R. H. Grubbs, J. Am. Chem. Soc. 2008, 130, 5874-5875.

[200] M. G. Scheibel, B. Askevold, F. W. Heinemann, E. J. Reijerse, B. de Bruin, S. Schneider, Nature Chem. 2012, 4, 552-558.

[201] R. Ghosh, M. Kanzelberger, T. J. Emge, G. S. Hall, A. S. Goldman, Organometallics 2006, 25, 5668-5671.

[202] D. W. Lee, W. C. Kaska, C. M. Jensen, Organometallics 1998, 17, 1-3.

[203] A. S. Goldman, K. I. Goldberg in ACS Symposium Series 885: Activation and Functionalization of $\mathrm{C}$-H Bonds (Eds.: A. S. Goldman, K. I. Goldberg), American Chemical Society, Washington DC, 2004, pp. 1-43.

[204] B. Askevold, A. Friedrich, M. R. Buchner, B. Lewall, A. C. Filippou, E. Herdtweck, S. Schneider, J. Organomet. Chem. 2013, 744, 35-40.

[205] S. Park, A. L. Rheingold, D. M. Roundhill, Organometallics 1991, 10, 615-623.

[206] A. N. Marziale, E. Herdtweck, J. Eppinger, S. Schneider, Inorg. Chem. 2009, 48, 3699-3709.

[207] A. Friedrich, M. Drees, M. Käss, E. Herdtweck, S. Schneider, Inorg. Chem. 2010, $49,5482-5494$. 
[208] B. de Bruin, P. H. M. Budzelaar, A. W. Gal, Angew. Chem. Int. Ed. 2004, 43, $4142-4157$.

[209] C. Tejel, Top. Organomet. Chem. 2007, 22, 97-124.

[210] M. R. Kelley, J.-U. Rohde, Chem. Commun. 2012, 48, 2876-2878.

[211] Z. Huang, P. S. White, M. Brookhart, Nature 2010, 465, 598-601.

[212] C. Tejel, M. P. del Río, M. A. Ciriano, E. J. Reijerse, F. Hartl, S. Záliš, D. G. H. Hetterscheid, N. Tsichilis i Spithas, N. Tsichlis i Spithas, B. de Bruin, Chem. Eur. J. 2009, 15, 11878-11889.

[213] J. M. Praetorius, D. P. Allen, R. Wang, J. D. Webb, F. Grein, P. Kennepohl, C. M. Crudden, J. Am. Chem. Soc. 2008, 130, 3724-3725.

[214] D. W. Lee, C. M. Jensen, D. Morales-Morales, Organometallics 2003, 22, 47444749.

[215] H. Lebel, C. Ladjel, F. Bélanger-Gariépy, F. Schaper, J. Organomet. Chem. 2008, 693, 2645-2648.

[216] A. Y. Verat, H. Fan, M. Pink, Y.-S. Chen, K. G. Caulton, Chem. Eur. J. 2008, 14, 7680-7686.

[217] C. Frech, L. Shimon, D. Milstein, Helv. Chim. Acta 2006, 89, 1730-1739.

[218] L. Bonomo, E. Solari, R. Scopelliti, C. Floriani, Angew. Chem. Int. Ed. 2001, 113, $2597-2599$.

[219] J. S. Pap, S. DeBeer George, J. F. Berry, Angew. Chem. Int. Ed. 2008, 47, 1010210105.

[220] A. K. Musch Long, R. P. Yu, G. H. Timmer, J. F. Berry, J. Am. Chem. Soc. 2010, 132, 12228-12230.

[221] J. Schöffel, N. Šušnjar, S. Nückel, D. Sieh, P. Burger, Eur. J. Inorg. Chem. 2010, 2010, 4911-4915.

[222] M. G. Scheibel, Y. Wu, A. C. Stückl, L. Krause, E. Carl, D. Stalke, B. de Bruin, S. Schneider, J. Am. Chem. Soc. 2013, 5, 17719-17722. 
[223] Y. Wu, Master Thesis, Friedrich-Alexander University Erlangen-Nuremberg, 2012.

[224] M. Feller, E. Ben-Ari, T. Gupta, L. J. W. Shimon, G. Leitus, Y. Diskin-Posner, L. Weiner, D. Milstein, Inorg. Chem. 2007, 46, 10479-10490.

[225] B. Goodman, J. Raynor, Adv. Inorg. Chem. Radiochem. 1970, 13, 135-362.

[226] J. J. Warren, T. A. Tronic, J. M. Mayer, Chem. Rev. 2010, 110, 6961-7001.

[227] H. Henderickx, G. Kwakkenbos, A. Peters, J. van der Spoel, K. de Vries, Chem. Commun. 2003, 2050-2051.

[228] H. M. L. Davies, J. R. Manning, Nature 2008, 451, 417-424.

[229] K. W. Fiori, C. G. Espino, B. H. Brodsky, J. Du Bois, Tetrahedron 2009, 65, 3042-3051.

[230] J. L. Jeffrey, R. Sarpong, Chem. Sci. 2013, 4, 4092-4106.

[231] P. M. Wehn, J. Du Bois, J. Am. Chem. Soc. 2002, 124, 12950-12951.

[232] A. Hinman, J. Du Bois, J. Am. Chem. Soc. 2003, 125, 11510-11511.

[233] J. J. Fleming, J. Du Bois, J. Am. Chem. Soc. 2006, 128, 3926-3927.

[234] J.-L. Liang, S.-X. Yuan, J.-S. Huang, C.-M. Che, J. Org. Chem. 2004, 69, 36103619.

[235] V. Lyaskovskyy, A. I. O. Suarez, H. Lu, H. Jiang, X. P. Zhang, B. de Bruin, J. Am. Chem. Soc. 2011, 133, 12264-12273.

[236] J. F. Berry, Dalton Trans. 2012, 41, 700-713.

[237] S.-M. Au, J.-S. Huang, W.-Y. Yu, W.-H. Fung, C.-M. Che, J. Am. Chem. Soc. 1999, 121, 9120-9132.

[238] A. I. Olivos Suarez, H. Jiang, X. P. Zhang, B. de Bruin, Dalt. Trans. 2011, 40, $5697-5705$.

[239] D. L. J. Broere, B. de Bruin, J. N. H. Reek, M. Lutz, S. Dechert, J. I. van der Vlugt, J. Am. Chem. Soc. 2014, 136, 11574-11577. 
[240] G. H. Timmer, J. F. Berry, Chem. Sci. 2012, 3, 3038-3052.

[241] A. R. Corcos, A. K. M. Long, I. a. Guzei, J. F. Berry, Eur. J. Inorg. Chem. 2013, 2013, 3808-3811.

[242] D. M. King, F. Tuna, E. J. L. McInnes, J. McMaster, W. Lewis, A. J. Blake, S. T. Liddle, Nature Chem 2013, 5, 482-488.

[243] Y.-H. Chang, Y. Nakajima, H. Tanaka, K. Yoshizawa, F. Ozawa, J. Am. Chem. Soc. 2013, 135, 11791-11794.

[244] J. Zhao, A. S. Goldman, J. F. Hartwig, Science 2005, 307, 1080-1082.

[245] M. Kanzelberger, X. Zhang, T. J. Emge, A. S. Goldman, J. Zhao, C. Incarvito, J. F. Hartwig, J. Am. Chem. Soc. 2003, 13644-13645.

[246] D. Rais, R. G. Bergman, Chem. Eur. J. 2004, 10, 3970-3978.

[247] J. R. Fulton, M. W. Bouwkamp, R. G. Bergman, J. Am. Chem. Soc. 2000, 122, 8799-8800.

[248] M. G. Scheibel, I. Klopsch, H. Wolf, P. Stollberg, D. Stalke, S. Schneider, Eur. J. Inorg. Chem. 2013, 2013, 3836-3839.

[249] W. Baratta, C. Mealli, E. Herdtweck, A. Ienco, S. A. Mason, P. Rigo, J. Am. Chem. Soc. 2004, 126, 5549-5562.

[250] N. Tsvetkov, M. Pink, H. Fan, J.-H. Lee, K. G. Caulton, Eur. J. Inorg. Chem. 2010, 2010, 4790-4800.

[251] H. D. Empsall, E. M. Hyde, R. Markham, W. S. McDonald, M. C. Norton, B. L. Shaw, B. Weeks, J. Chem. Soc. Chem. Commun. 1977, 589-590.

[252] N. A. Al-Salem, H. D. Empsall, R. Markham, B. L. Shaw, B. Weeks, J. Chem. Soc. Dalt. Trans. 1979, 1972-1982.

[253] B. M. Gardner, G. Balázs, M. Scheer, F. Tuna, E. J. L. McInnes, J. McMaster, W. Lewis, A. J. Blake, S. T. Liddle, Angew. Chem. Int. Ed. 2014, 4484-4488.

[254] B. L. Tran, M. P. Washington, D. a. Henckel, X. Gao, H. Park, M. Pink, D. J. Mindiola, Chem. Commun. 2012, 48, 1529-1531. 
[255] P. J. McKarns, G. P. A. Yap, A. L. Rheingold, C. H. Winter, Inorg. Chem. 1996, 2, 5968-5969.

[256] R. Thompson, C.-H. Chen, M. Pink, G. Wu, D. J. Mindiola, J. Am. Chem. Soc. 2014, 6917, 15-18.

[257] G. Parkin, A. V. Asselt, D. J. Leahy, L. Whinnery, N. G. Hua, R. W. Quan, L. M. Henling, W. P. Schaefer, B. D. Santarsiero, J. E. Bercaw, Inorg. Chem. 1992, 31, $82-85$.

[258] A. J. Keane, P. Y. Zavalij, L. R. Sita, J. Am. Chem. Soc. 2013, 135, 9580-9583.

[259] J. S. Freundlich, R. R. Schrock, C. C. Cummins, W. M. Davis, N. Taciz, J. Am. Chem. Soc. 1994, 116, 1987-1988.

[260] C. C. Cummins, R. R. Schrock, W. M. Davis, Inorg. Chem. 1994, 33, 1448-1457.

[261] D. V. Yandulov, R. R. Schrock, J. Am. Chem. Soc. 2002, 124, 6252-6253.

[262] R. R. Schrock, T. E. Glassman, M. G. Vale, J. Am. Chem. Soc. 1991, 7, 725-726.

[263] M. R. Reithofer, R. R. Schrock, , P. Müller, J. Am. Chem. Soc. 2010, 132, 83498358.

[264] P. C. Bevan, J. Chatt, J. R. Dilworth, R. A. Henderson, G. J. Leigh, J. Chem. Soc. Dalton Trans. 1982, 821-824.

[265] J. Chatt, J. R. Dilworth, J. Chem. Soc. Chem. Commun. 1975, 983-984.

[266] R. R. Schrock, T. E. Glassman, M. G. Vale, M. Kol, J. Am. Chem. Soc. 1993, 115, 1760-1772.

[267] Y. Alias, S. K. Ibrahim, M. A. Queiros, A. Fonseca, J. Talarmin, F. Volant, C. J. Pickett, J. Chem. Soc. Dalt. Trans. 1997, 4807-4816.

[268] C. M. Lutz, S. R. Wilson, P. A. Shapley, Organometallics 2005, 24, 3350-3353.

[269] M. H. V. Huynh, P. S. White, K. D. John, T. J. Meyer, Angew. Chem. Int. Ed. 2001, 40, 4049-4051.

[270] A. C. Bowman, S. C. Bart, F. W. Heinemann, K. Meyer, P. J. Chirik, Inorg. Chem. 2009, 48, 5587-5589. 
[271] E. A. Mader, V. W. Manner, T. F. Markle, A. Wu, J. a. Franz, J. M. Mayer, J. Am. Chem. Soc. 2009, 131, 4335-4345.

[272] M. Herberhold, Comments Inorg. Chem. 1988, 7, 53-72.

[273] B. F. G. Johnson, B. L. Haymore, R. R. Dilworth in Comprehensive coordination chemistry (Eds.: G. E. Wilkinson, R. D. Gillards, J. A. McCleverty), Pergamon, New York, 1987, pp. 99-159.

[274] K. K. Pandey in Prog. Inorg. Chem., John Wiley \& Sons, Inc., 2007, pp. 445-502.

[275] P. Rademacher in Strukt. Org. Moleküle, Wiley-VCH Verlag GmbH \& Co. KGaA, 2005.

[276] J. H. Enemark, R. D. Feltham, Coord. Chem. Rev. 1974, 13, 339-406.

[277] J. Bendix, K. Meyer, T. Weyhermüller, E. Bill, N. Metzler-Nolte, K. Wieghardt, Inorg. Chem. 1998, 37, 1767-1775.

[278] J. F. Berry, Comments Inorg. Chem. 2009, 30, 28-66.

[279] P. Hummel, H. B. Gray, Coord. Chem. Rev. 2007, 251, 554-556.

[280] P. Hummel, J. Winkler, H. Gray, Theor. Chem. Acc. 2008, 119, 35-38.

[281] S. F. Vyboishchikov, G. Frenking, Theor. Chem. Acc. 1999, 102, 300-308.

[282] D. F. Evans, J. Chem. Soc. 1959, 2003-2005.

[283] S. K. Sur, J. Magn. Reson. 1989, 82, 169-173.

[284] D. H. Grant, J. Chem. Educ. 1995, 72, 39-40.

[285] A. Pacault, J. Hoarau, A. Marchand in Adv. Chem. Phys., John Wiley \& Sons, Inc., 2007, pp. 171-238.

[286] W. Klemm, Zeitschrift für Anorg. und Allg. Chemie 1940, 244, 377-396.

[287] W. Klemm, Zeitschrift für Anorg. und Allg. Chemie 1941, 246, 347-362.

[288] G. R. Fulmer, A. J. M. Miller, N. H. Sherden, H. E. Gottlieb, A. Nudelman, B. M. Stoltz, J. E. Bercaw, K. I. Goldberg, Organometallics 2010, 29, 2176-2179. 
[289] Z. E. Clarke, P. T. Maragh, T. P. Dasgupta, D. G. Gusev, A. J. Lough, K. AbdurRashid, Organometallics 2006, 25, 4113-4117.

[290] J. Abad, Inorg. Chim. Acta 1986, 121, 213-217.

[291] A. Martinsen, J. Songstad, Acta Chem. Scondinavica A 1977, 31, 645-650.

[292] J.-M. Lalancette, G. Rollin, P. Dumas, Can. J. Chem. 1972, 50, 3058-3062.

[293] J. A. Osborn, G. Wilkinson, J. J. Mrowca in Inorg. Synth., John Wiley \& Sons, Inc., 2007, pp. 77-79.

[294] S. H. Babcock, H. P. Lankelma, E. Vopicka in Inorg. Synth., John Wiley \& Sons, Inc., 2007, pp. 10-11.

[295] M. Brookhart, B. Grant, A. F. Volpe, Organometallics 1992, 11, 3920-3922.

[296] N. A. Giffin, M. Makramalla, A. D. Hendsbee, K. N. Robertson, C. Sherren, C. C. Pye, J. D. Masuda, J. a. C. Clyburne, Org. Biomol. Chem. 2011, 9, 3672-3680.

[297] V. W. Manner, T. F. Markle, J. H. Freudenthal, J. P. Roth, J. M. Mayer, Chem. Commun. 2008, 246, 256-258. 
Part VI.

Appendix 


\section{Index of all numerated compounds}

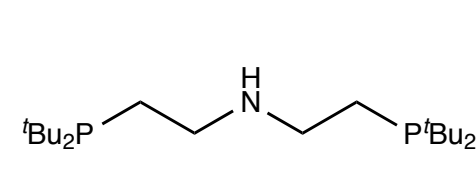

1

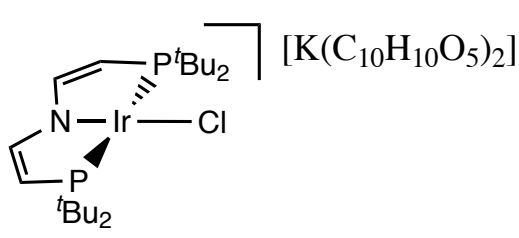

5

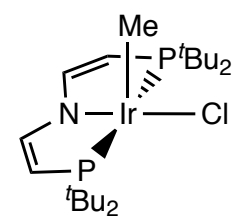

9

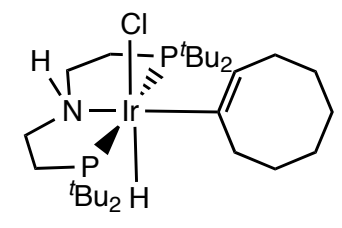

2

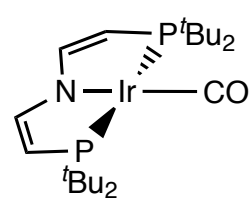

6

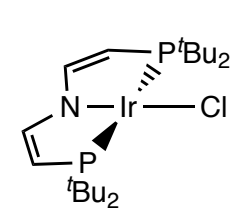

3

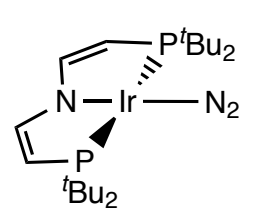

7

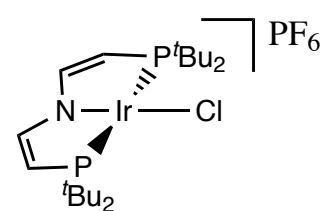

4

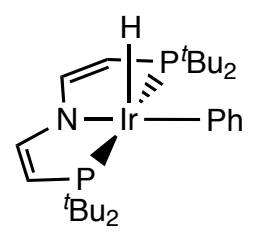

8

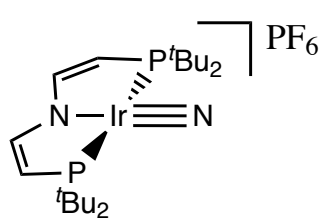

12 


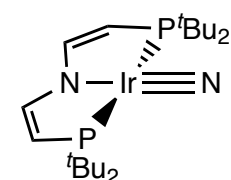

13

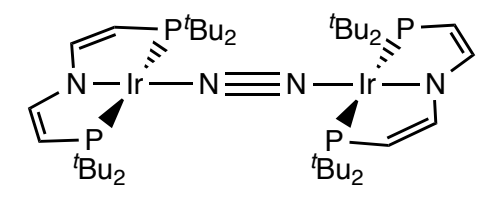

14

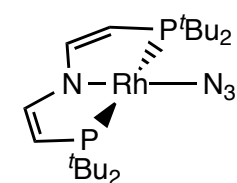

15

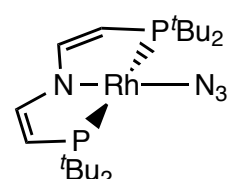

16

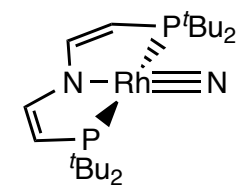

17

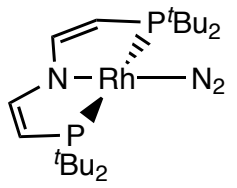

18

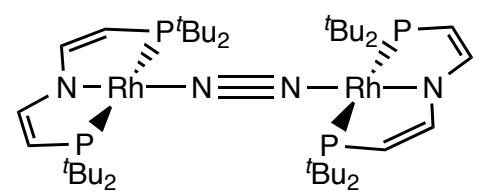

19

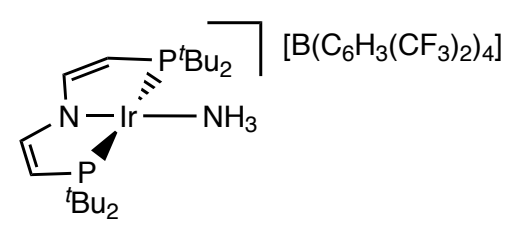

20

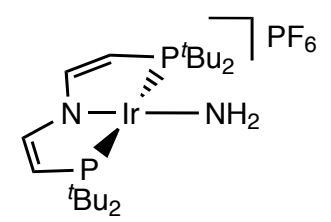

21

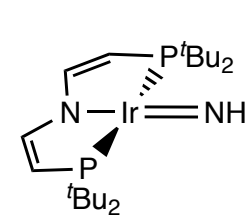

22

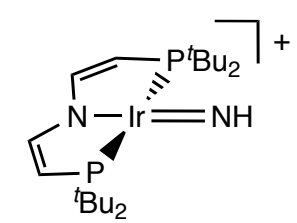

23

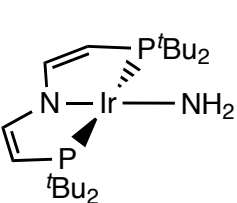

24

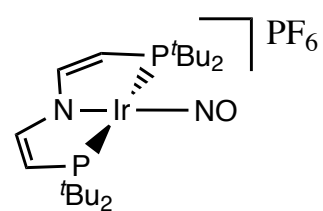

25

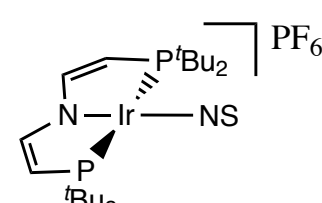

26

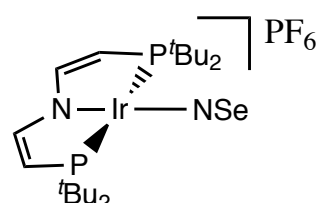

27 


\section{List of Abbreviations}

\begin{tabular}{|c|c|}
\hline$\delta$ & chemical shift \\
\hline$\Delta H_{0}^{f}$ & heat of formation at $25^{\circ} \mathrm{C}, 298 \mathrm{~K}$ \\
\hline$\mu_{B}$ & Bohr magneton \\
\hline$\mu_{e f f}$ & effective magnetization \\
\hline BDE & bond dissociation energy \\
\hline br & broad \\
\hline${ }^{t} \mathrm{Bu}$ & tert-butyl \\
\hline calcd. & calculated \\
\hline $\mathrm{COE}$ & cis-cyclooctene \\
\hline $\mathrm{CV}$ & cyclic voltammetry \\
\hline $\mathrm{d}$ & doublet \\
\hline DCM & dichloromethane \\
\hline DFT & density functional theory \\
\hline e.g. & example given \\
\hline ENDOR & electron nuclear double resonance \\
\hline $\begin{array}{l}\text { EPR } \\
\text { equiv }\end{array}$ & $\begin{array}{l}\text { electron paramagnetic resonance } \\
\text { equivalents }\end{array}$ \\
\hline ESI & electron spray ionization \\
\hline $\exp$ & experimental \\
\hline $\mathrm{Fc}$ & ferrocene \\
\hline $\mathrm{Fc}^{+}$ & ferrocenium cation \\
\hline FT & fourrier transform \\
\hline h & hour \\
\hline HAT & Hydrogen atom abstraction \\
\hline HFI & hyperfine interaction \\
\hline $\mathrm{HOMO}$ & highest occupied molecular orbital \\
\hline hs & high spin \\
\hline${ }^{i} \operatorname{Pr}$ & isopropyl \\
\hline
\end{tabular}




\begin{tabular}{|c|c|}
\hline IR & infrared \\
\hline is & intermediate spin \\
\hline i. vac. & in vacuo \\
\hline ls & low spin \\
\hline LUMO & lowest unoccupied molecular orbital \\
\hline $\mathrm{m}$ & multiplet \\
\hline $\mathrm{m}$ & medium \\
\hline $\mathrm{Me}$ & methyl \\
\hline Mes & mesithyl, 2,4,6-trimethylphenyl \\
\hline $\mathrm{MO}$ & molecular orbital \\
\hline$M$ & molar \\
\hline NMR & nuclear magnetic resonance \\
\hline NQI & nuclear quadrupole interactions \\
\hline Otf & trifluoromethanesulfonate \\
\hline PDI & pyridine-2,6-bis $(N$-(2,6-diisopropylphenyl)ethan-1-imine) \\
\hline $\mathrm{Ph}$ & phenyl \\
\hline $\mathrm{PNP}^{\prime}$ & bis(di-tert-butylphosphinoethylene)amide \\
\hline ppm & parts per million \\
\hline q & quartet \\
\hline RT & room temperature \\
\hline S & spin \\
\hline s & singlet \\
\hline s & strong \\
\hline sept & septet \\
\hline SOC & spin orbit coupling \\
\hline SOMO & singly occupied molecular orbital \\
\hline SQUID & superconducting quantum interference device \\
\hline $\mathrm{t}$ & triplet \\
\hline TEMPO & tetramethylpiperidine- $N$-oxid \\
\hline THF & tetrahydrofurane \\
\hline $\mathrm{TP}$ & hydridotris(pyrazolyl)borate \\
\hline vs & very strong \\
\hline vs. & versus \\
\hline $\mathrm{vw}$ & very weak \\
\hline $\mathrm{W}$ & weak \\
\hline WBI & Wiberg bond index \\
\hline
\end{tabular}




\section{Crystallographic Details}

\section{1. $\left[\operatorname{Ir}\left\{\mathrm{N}\left(\mathrm{CHCHP}^{t} \mathrm{Bu}_{2}\right)_{2}\right\} \mathrm{Cl}\right] \mathrm{PF}_{6}$ (4)}

Empirical formula

Formula weight

Temperature

Wavelength

Crystal system, space group

Unit cell dimensions

Volume

Z, Calculated density

Absorption coefficient

$\mathrm{F}(000)$

Crystal size

$\theta$ range for data collection

Limiting indices

Reflections collected / unique

Completeness to $\theta=25.38$

Absorption correction

Max. and min. transmission

Refinement method

Data / restraints / parameters

Goodness-of-fit on $\mathrm{F}^{2}$

Final $R$ indices $[\mathrm{I}>2 \sigma(\mathrm{I})]$

$\mathrm{R}$ indices (all data)

Largest diff. peak and hole
C24 H48 Cl F6 Ir N O P3

801.21

123(1) K

$0.71073 \AA$

Monoclinic, $\mathrm{P} 22_{1} / \mathrm{n}$

$\mathrm{a}=14.5723(4) \AA \alpha=90^{\circ}$

$\mathrm{b}=14.7565(5) \AA \beta=109.7116(15)^{\circ}$

$\mathrm{c}=15.6793(5) \AA \gamma=90^{\circ}$

3174.05(17) $\AA^{3}$

$4,1.677 \mathrm{Mg} / \mathrm{m}^{3}$

$4.496 \mathrm{~mm}^{-1}$

1600

$0.38 \times 0.25 \times 0.18 \mathrm{~mm}^{3}$

1.65 to $25.38^{\circ}$

$-17 \leq \mathrm{h} \leq 17,-17 \leq \mathrm{k} \leq 17,-18 \leq \mathrm{l} \leq 18$

$80345 / 5807$ [R(int $)=0.0373]$

$99.8 \%$

Semi-empirical from equivalents

0.7452 and 0.5462

Full-matrix least-squares on $\mathrm{F}^{2}$

$5807 / 0 / 346$

1.047

$\mathrm{R} 1=0.0147, \mathrm{wR} 2=0.0324$

$\mathrm{R} 1=0.0172, \mathrm{wR} 2=0.0333$

0.574 and $-0.449 \mathrm{e}^{-3}$ 


\section{2. $\left[\mathrm{K}\left(\mathrm{C}_{10} \mathrm{H}_{10} \mathrm{O}_{5}\right)_{2}\right]\left[\operatorname{Ir}\left\{\mathrm{N}\left(\mathrm{CHCHP}^{t} \mathrm{Bu}_{2}\right)_{2}\right\} \mathrm{Cl}\right]$ (5)}

Empirical formula

Formula weight

Temperature

Wavelength

Crystal system, space group

Unit cell dimensions

Volume

Z, Calculated density

Absorption coefficient

$\mathrm{F}(000)$

Crystal size

$\theta$ range for data collection

Limiting indices

Reflections collected / unique

Completeness to $\theta=19.665$

Absorption correction

Max. and min. transmission

Refinement method

Data / restraints / parameters

Goodness-of-fit on $\mathrm{F}^{2}$

Final $\mathrm{R}$ indices $[\mathrm{I}>2 \sigma(\mathrm{I})]$

$\mathrm{R}$ indices (all data)

Extinction coefficient
C40 H80 Cl Ir K N O10 P2

1063.74

100(2) K

$0.56086 \AA$

Orthorhombic, $\mathrm{P}$ b c n

$\mathrm{a}=20.279(2) \AA \alpha=90^{\circ}$

$\mathrm{b}=18.474(2) \AA \beta=90^{\circ}$

$\mathrm{c}=26.693(3) \AA \gamma=90^{\circ}$

10000.1(18) $\AA^{3}$

$8,1.413 \mathrm{Mg} / \mathrm{m}^{3}$

$1.586 \mathrm{~mm}^{-1}$

4400

$0.103 \times 0.103 \times 0.097 \mathrm{~mm}^{3}$

1.585 to $20.301^{\circ}$

$-20 \leq \mathrm{h} \leq 25,-21 \leq \mathrm{k} \leq 22,-32 \leq \mathrm{l} \leq 26$

$158816 / 9805$ [R(int $)=0.0787]$

$99.8 \%$

Semi-empirical from equivalents

0.7445 and 0.6775

Full-matrix least-squares on $\mathrm{F}^{2}$

$9805 / 156 / 539$

1.094

$\mathrm{R} 1=0.0303, \mathrm{wR} 2=0.0497$

$\mathrm{R} 1=0.0511, \mathrm{wR} 2=0.0533$

0.492 and $-0.760 \mathrm{e}^{-3}$ 


\section{3. $\left[\operatorname{Ir}\left\{\mathrm{N}\left(\mathrm{CHCHP}{ }^{t} \mathrm{Bu}_{2}\right)_{2}\right\}(\mathrm{CO})\right](6)$}

Empirical formula

Formula weight

Temperature

Wavelength

Crystal system, space group

Unit cell dimensions

Volume

Z, Calculated density

Absorption coefficient

$\mathrm{F}(000)$

Crystal size

$\theta$ range for data collection

Limiting indices

Reflections collected / unique

Completeness to $\theta=28.70$

Absorption correction

Max. and min. transmission

Refinement method

Data / restraints / parameters

Goodness-of-fit on $\mathrm{F}^{2}$

Final R indices $[\mathrm{I}>2 \sigma(\mathrm{I})]$

$\mathrm{R}$ indices (all data)

Largest diff. peak and hole
C21 H40 Ir N O P2

576.68

150(2) K

$0.71073 \AA$

Monoclinic, P2(1)/c

$\mathrm{a}=7.9010(3) \AA \alpha=90^{\circ}$

$\mathrm{b}=26.8610(12) \AA \beta=106.864(5)^{\circ}$

$\mathrm{c}=11.8140(10) \AA \gamma=90^{\circ}$

2399.4(2) $\AA^{3}$

$4,1.596 \mathrm{Mg} / \mathrm{m}^{3}$

$5.708 \mathrm{~mm}^{-1}$

1152

$0.24 \times 0.16 \times 0.04 \mathrm{~mm}^{3}$

3.03 to $28.70^{\circ}$

$-10 \leq \mathrm{h} \leq 10,-36 \leq \mathrm{k} \leq 36,-15 \leq \mathrm{l} \leq 15$

$69417 / 6192[\mathrm{R}($ int $)=0.0524]$

$99.9 \%$

Semi-empirical from equivalents

1.0 and 0.612

Full-matrix least-squares on $\mathrm{F}^{2}$ $6192 / 0 / 247$

$\mathrm{R} 1=0.0199, \mathrm{wR} 2=0.0400$

$\mathrm{R} 1=0.0248, \mathrm{wR} 2=0.0413$

0.916 and -0.810 e $\AA^{-3}$ 


\section{4. $\left[\operatorname{Ir}\left\{\mathrm{N}\left(\mathrm{CHCHP}{ }^{t} \mathrm{Bu}_{2}\right)_{2}\right\}\left(\mathrm{N}_{2}\right)\right](7)$}

Empirical formula

Formula weight

Temperature

Wavelength

Crystal system, space group

Unit cell dimensions

Volume

Z, Calculated density

Absorption coefficient

$\mathrm{F}(000)$

Crystal size

$\theta$ range for data collection

Limiting indices

Reflections collected / unique

Completeness to $\theta=28.70$

Absorption correction

Max. and min. transmission

Refinement method

Data / restraints / parameters

Goodness-of-fit on $\mathrm{F}^{2}$

Final $\mathrm{R}$ indices $[\mathrm{I}>2 \sigma(\mathrm{I})]$

$\mathrm{R}$ indices (all data)

Largest diff. peak and hole
C20 H40 Ir N3 P2

576.69

100(2) K

$0.71073 \AA$

Monoclinic, $\mathrm{P} 22_{1} / \mathrm{c}$

$\mathrm{a}=7.8440(6) \AA \alpha=90^{\circ}$

$\mathrm{b}=26.903(2) \AA \beta=106.640(2)^{\circ}$

$\mathrm{c}=11.8151(9) \AA \gamma=90^{\circ}$

2388.8(3) $\AA^{3}$

$4,1.603 \mathrm{Mg} / \mathrm{m}^{3}$

$5.732 \mathrm{~mm}^{-1}$

1152

$0.13 \times 0.09 \times 0.02 \mathrm{~mm}^{3}$

2.71 to $28.70^{\circ}$

$-10 \leq \mathrm{h} \leq 10,-27 \leq \mathrm{k} \leq 36,-13 \leq \mathrm{l} \leq 15$

$22146 / 6164$ [R(int) $=0.0286]$

$99.7 \%$

Semi-empirical from equivalents

0.746 and 0.583

Full-matrix least-squares on $\mathrm{F}^{2}$

$6164 / 0 / 247$

1.110

$\mathrm{R} 1=0.0280, \mathrm{wR} 2=0.0552$

$\mathrm{R} 1=0.0327, \mathrm{wR} 2=0.0566$

1.273 and $-2.467 \mathrm{e}^{-3}$ 


\section{5. $\left[\operatorname{Ir}\left\{\mathrm{N}\left(\mathrm{CHCHP}^{t} \mathrm{Bu}_{2}\right)_{2}\right\}(\mathrm{H})\left(\mathrm{C}_{6} \mathrm{H}_{5}\right)\right](8)$}

Empirical formula

Formula weight

Temperature

Wavelength

Crystal system, space group

Unit cell dimensions

Volume

Z, Calculated density

Absorption coefficient

$\mathrm{F}(000)$

Crystal size

$\theta$ range for data collection

Index ranges

Reflections collected

Independent reflections

Completeness to $\theta=19.665^{\circ}$

Absorption correction

Max. and min. transmission

Refinement method

Data / restraints / parameters

Goodness-of-fit on $\mathrm{F}^{2}$

Final $R$ indices $[\mathrm{I}>2 \operatorname{sigma}(\mathrm{I})]$

$\mathrm{R}$ indices (all data)

Extinction coefficient

Largest diff. peak and hole
C26 H46 Ir N P2

626.78

100(2) K

$0.56086 \AA$

Monoclinic, $\mathrm{P} 22_{1} / \mathrm{n}$

$\mathrm{a}=8.896(2) \AA \alpha=90^{\circ}$

$\mathrm{b}=29.428(7) \AA \quad \beta=100.058(5)^{\circ}$

$\mathrm{c}=10.601(2) \AA \gamma=90^{\circ}$

2732.5(11) $\AA^{3}$

$4,1.524 \mathrm{Mg} / \mathrm{m}^{3}$

$2.729 \mathrm{~mm}-1$

1264

$0.110 \times 0.070 \times 0.060 \mathrm{~mm}^{3}$

1.634 to $20.554^{\circ}$

$-11 \leq \mathrm{h} \leq 11,-36 \leq \mathrm{k} \leq 36,-13 \leq \mathrm{l} \leq 13$

24583

$5543[\mathrm{R}(\mathrm{int})=0.0561]$

$99.8 \%$

Semi-empirical from equivalents

0.5599 and 0.4793

Full-matrix least-squares on $\mathrm{F}^{2}$

$5543 / 89 / 287$

1.134

$\mathrm{R} 1=0.0494, \mathrm{wR} 2=0.1477$

$\mathrm{R} 1=0.0615, \mathrm{wR} 2=0.1545$

$\mathrm{n} / \mathrm{a}$

1.428 and -3.398 e $\AA^{-3}$ 


\section{6. $\left[\operatorname{Ir}\left\{\mathrm{N}\left(\mathrm{CHCHP}^{t} \mathrm{Bu}_{2}\right)_{2}\right\}\left(\mathrm{O}_{2}\right)\right](10)$}

Empirical formula

Formula weight

Temperature

Wavelength

Crystal system, space group

Unit cell dimensions

Volume

Z, Calculated density

Absorption coefficient

$\mathrm{F}(000)$

Crystal size

$\theta$ range for data collection

Index ranges

Reflections collected

Independent reflections

Completeness to $\theta=19.665^{\circ}$

Absorption correction

Max. and min. transmission

Refinement method

Data / restraints / parameters

Goodness-of-fit on $\mathrm{F}^{2}$

Final R indices $[\mathrm{I}>2 \sigma(\mathrm{I})]$

$\mathrm{R}$ indices (all data)

Extinction coefficient

Largest diff. peak and hole
C20 H40 Ir N O2 P2

580.67

100(2) K

$0.56086 \AA$

Monoclinic, C $2 / \mathrm{m}$

$\mathrm{a}=13.917(3) \AA \alpha=90^{\circ}$

$\mathrm{b}=7.7210(15) \AA \quad \beta=111.39(3)^{\circ}$

$\mathrm{c}=11.629(2) \AA \gamma=90^{\circ}$

$1163.5(5) \AA^{3}$

$2,1.657 \mathrm{Mg} / \mathrm{m}^{3}$

$3.203 \mathrm{~mm}^{-1}$

580

$0.090 \times 0.080 \times 0.060 \mathrm{~mm}^{3}$

2.381 to $23.612^{\circ}$

$-19 \leq \mathrm{h} \leq 19,-11 \leq \mathrm{k} \leq 11,-16 \leq \mathrm{l} \leq 16$

19582

$1891[\mathrm{R}(\mathrm{int})=0.0592]$

$99.9 \%$

Semi-empirical from equivalents

0.7459 and 0.6594

Full-matrix least-squares on $\mathrm{F}^{2}$

$1891 / 161 / 126$

1.064

$\mathrm{R} 1=0.0168, \mathrm{wR} 2=0.0370$

$\mathrm{R} 1=0.0168, \mathrm{wR} 2=0.0370$

$\mathrm{n} / \mathrm{a}$

1.260 and $-0.743 \mathrm{e} \AA^{-3}$ 


\section{7. $\left[\operatorname{Ir}\left\{\mathrm{N}\left(\mathrm{CHCHP}^{t} \mathrm{Bu}_{2}\right)_{2}\right\} \mathrm{N}\right] \mathrm{OTf}(12)$}

Empirical formula

Formula weight

Temperature

Wavelength

Crystal system, space group

Unit cell dimensions

Volume

Z, Calculated density

Absorption coefficient

$\mathrm{F}(000)$

Crystal size

$\theta$ range for data collection

Limiting indices

Reflections collected / unique

Completeness to $\theta=26.00$

Absorption correction

Max. and min. transmission

Refinement method

Data / restraints / parameters

Goodness-of-fit on $\mathrm{F}^{2}$

Final R indices [I $>2 \sigma(\mathrm{I})]$

$\mathrm{R}$ indices (all data)

Largest diff. peak and hole
C43 H82 Cl2 F6 Ir2 N4 O6 P4 S2

1508.43

100(2) K

$0.71073 \AA$

Triclinic, $\mathrm{P} \overline{1}$

$\mathrm{a}=15.0826(3) \AA \quad \alpha=62.5630(10)^{\circ}$

$\mathrm{b}=15.0937(3) \AA \beta=78.9770(10)^{\circ}$

$\mathrm{c}=16.1407(4) \AA \gamma=62.6930(10)^{\circ}$ $2896.79(11) \AA^{3}$

$2,1.729 \mathrm{Mg} / \mathrm{m}^{3}$

$4.929 \mathrm{~mm}^{-1}$

1500

$0.40 \times 0.22 \times 0.20 \mathrm{~mm}^{3}$

2.05 to $28.70^{\circ}$

$-20 \leq \mathrm{h} \leq 20,-19 \leq \mathrm{k} \leq 20,-21 \leq \mathrm{l} \leq 21$

$52472 / 14124[\mathrm{R}(\mathrm{int})=0.0465]$

$99.4 \%$

Semi-empirical from equivalents

0.746 and 0.572

Full-matrix least-squares on $\mathrm{F}^{2}$ $14124 / 0 / 646$ 1.025

$\mathrm{R} 1=0.0358, \mathrm{wR} 2=0.0828$

$\mathrm{R} 1=0.0570, \mathrm{wR} 2=0.0929$

3.767 and $-2.409 \mathrm{e}^{-3}$ 


\section{8. $\left[\operatorname{Ir}\left\{\mathrm{N}\left(\mathrm{CHCHP}^{t} \mathrm{Bu}_{2}\right)_{2}\right\}\right]_{2}\left(\mathrm{~N}_{2}\right)$}

Empirical formula

Formula weight

Temperature

Wavelength

Crystal system, Space group

Unit cell dimensions

Volume

Z, Calculated density

Absorption coefficient

$\mathrm{F}(000)$

Crystal size

$\theta$ range for data collection

Index ranges

Reflections collected

Independent reflections

Completeness to $\theta$

Absorption correction

Max. and min. transmission

Refinement method

Data / restraints / parameters

Goodness-of-fit on F2

Final R indices $[\mathrm{I}>2 \sigma(\mathrm{I})]$

$\mathrm{R}$ indices (all data)

Largest diff. peak and hole
C40 H80 Ir2 N4 P4

1125.36

100(2) K

$0.71073 \AA$

Triclinic, $\mathrm{P} \overline{1}$

$\mathrm{a}=11.5257(10) \AA \alpha=79.173(2)^{\circ}$

$\mathrm{b}=13.0336(11) \AA \beta=89.524(2)^{\circ}$

$\mathrm{c}=17.1075(14) \AA \gamma=68.544(2)^{\circ}$

2344.1(3) $\AA^{3}$

$2,1.594 \mathrm{Mg} / \mathrm{m}^{3}$

$5.838 \mathrm{~mm}^{-1}$

1124

$0.14 \times 0.10 \times 0.08 \mathrm{~mm}^{3}$

2.26 to $28.70^{\circ}$

$-15 \leq \mathrm{h} \leq 15,-17 \leq \mathrm{k} \leq 17,-22 \leq \mathrm{l} \leq 23$

41721

$12123[\mathrm{R}(\mathrm{int})=0.0373]$

$=28.70^{\circ} 99.9 \%$

Semi-empirical from equivalents

0.746 and 0.633

Full-matrix least-squares on F2

$$
12123 / 0 / 475
$$

1.025

$\mathrm{R} 1=0.0249, \mathrm{wR} 2=0.0501$

$\mathrm{R} 1=0.0360, \mathrm{wR} 2=0.0539$

1.160 and $-0.989 \mathrm{e}^{3}$ 


\section{9. $\left[\mathrm{Rh}\left\{\mathrm{HN}\left(\mathrm{CH}_{2} \mathrm{CH}_{2} \mathrm{P}^{t} \mathrm{Bu}_{2}\right)_{2}\right\}\left(\mathrm{PPh}_{3}\right)\right] \mathrm{Cl}$}

Empirical formula

Formula weight

Temperature

Wavelength

Crystal system, Space group

Unit cell dimensions

Volume

Z, Calculated Density

Absorption coefficient

$\mathrm{F}(000)$

Crystal size

$\theta$ range for data collection

Index ranges

Reflections collected

Independent reflections

Completeness to $\theta=19.665^{\circ}$

Absorption correction

Max. and min. transmission

Refinement method

Data / restraints / parameters

Goodness-of-fit on $\mathrm{F}^{2}$

Final R indices $[\mathrm{I}>2 \sigma(\mathrm{I})]$

$\mathrm{R}$ indices (all data)

Extinction coefficient

Largest diff. peak and hole
C38 H60 Cl N P3 Rh

762.14

100(2) K

$0.56086 \AA$

Triclinic, $\mathrm{P} \overline{1}$

$\mathrm{a}=10.1464(10) \AA \quad \alpha=77.982(10)^{\circ}$

$\mathrm{b}=10.4691(10) \AA \beta=81.91(2)^{\circ}$

$\mathrm{c}=19.064(2) \AA \gamma=75.721(10)^{\circ}$

1911.0(4) $\AA^{3}$

$2,1.325 \mathrm{Mg} / \mathrm{m}^{3}$

$0.355 \mathrm{~mm}^{-1}$

804

$0.10 \times 0.06 \times 0.05 \mathrm{~mm}^{3}$

0.866 to $20.582^{\circ}$

$-12 \leq \mathrm{h} \leq 12,-12 \leq \mathrm{k} \leq 13,0 \leq \mathrm{l} \leq 23$

7656

$7656[\mathrm{R}($ int $)=0.0443]$

$99.5 \%$

Semi-empirical from equivalents

0.744711 and 0.562901

Full-matrix least-squares on $\mathrm{F}^{2}$

$7656 / 0 / 414$

1.051

$\mathrm{R} 1=0.0478, \mathrm{wR} 2=0.0921$

$\mathrm{R} 1=0.0676, \mathrm{wR} 2=0.0992$

$\mathrm{n} / \mathrm{a}$

0.732 and -0.466 e $\AA^{-3}$ 


\subsection{0. $\left[\operatorname{Rh}\left\{\mathrm{N}\left(\mathrm{CHCHP}^{t} \mathrm{Bu}_{2}\right)_{2}\right\} \mathrm{Cl}\right](15)$}

Empirical formula

Formula weight

Temperature

Wavelength

Crystal system, Space Group

Unit cell dimensions

Volume

Z, Calculated Density

Absorption coefficient

$\mathrm{F}(000)$

Crystal size

$\theta$ range for data collection

Index ranges

Reflections collected

Independent reflections

Completeness to $\theta=25.242^{\circ}$

Absorption correction

Max. and min. transmission

Refinement method

Data / restraints / parameters

Goodness-of-fit on $\mathrm{F}^{2}$ v1.085

Final R indices $[\mathrm{I}>2 \sigma(\mathrm{I})]$

$\mathrm{R}$ indices (all data)

Extinction coefficient

Largest diff. peak and hole
C20 H40 Cl N P2 Rh

494.83

100(2) K

$0.71073 \AA$

Monoclinic, P 2/c

$$
\mathrm{a}=11.452(2) \AA \quad \alpha=90^{\circ}
$$

$\mathrm{b}=8.527(2) \AA \beta=113.31(2)^{\circ}$

$\mathrm{c}=13.432(3) \AA \gamma=90^{\circ}$

1204.6(5) $\AA^{3}$

$2,1.364 \mathrm{Mg} / \mathrm{m}^{3}$

$0.957 \mathrm{~mm}^{-1}$

518

$0.09 \times 0.07 \times 0.02 \mathrm{~mm}^{3}$

1.936 to $30.520^{\circ}$

$-16 \leq \mathrm{h} \leq 16,-12 \leq \mathrm{k} \leq 12,-19 \leq \mathrm{l} \leq 19$

33444

$3683[\mathrm{R}(\mathrm{int})=0.0267]$

$100.0 \%$

Semi-empirical from equivalents

0.4330 and 0.3999

Full-matrix least-squares on $\mathrm{F}^{2}$

$3683 / 0 / 122$

$$
\begin{array}{r}
\mathrm{R} 1=0.0183, \mathrm{wR} 2=0.0472 \\
\mathrm{R} 1=0.0202, \mathrm{wR} 2=0.0481 \\
\mathrm{n} / \mathrm{a} \\
0.685 \text { and }-0.205 \mathrm{e}^{-3}
\end{array}
$$




\subsection{1. $\left[\mathrm{Rh}\left\{\mathrm{N}\left(\mathrm{CHCHP}^{t} \mathrm{Bu}_{2}\right)_{2}\right\}\left(\mathrm{N}_{2}\right)\right]$ (18)}

Empirical formula

Formula weight

Temperature

Wavelength

Crystal system, space group

Unit cell dimensions

Volume

Z, Calculated density

Absorption coefficient

$\mathrm{F}(000)$

Crystal size

$\theta$ range for data collection

Limiting indices

Reflections collected / unique

Completeness to $\theta=19.665$

Absorption correction

Max. and min. transmission

Refinement method

Data / restraints / parameters

Goodness-of-fit on $\mathrm{F}^{2}$

Final R indices [I $>2 \sigma(\mathrm{I})]$

$\mathrm{R}$ indices (all data)

Extinction coefficient

Largest diff. peak and hole
C20 H40 N3 P2 Rh1

487.4

100(2) K

$0.56085 \AA$

Monoclinic, P 21/c

$\mathrm{a}=7.867(2) \AA \alpha=90^{\circ}$

$\mathrm{b}=27.050(6) \AA \beta=106.53(2)^{\circ}$

$\mathrm{c}=11.866(2) \AA \gamma=90^{\circ}$ $2420.8(9) \AA^{3}$

$4,1.337 \mathrm{Mg} / \mathrm{m}^{3}$

$0.450 \mathrm{~mm}^{-1}$

1024

$0.1 \times 0.05 \times 0.01 \mathrm{~mm}^{3}$

1.188 to $20.943^{\circ}$

$-9 \leq \mathrm{h} \leq 10,-34 \leq \mathrm{k} \leq 34,-15 \leq \mathrm{l} \leq 15$

$22081 / 5218$ [R(int $)=0.0552]$ $100.0 \%$

Semi-empirical from equivalents

0.7445 and 0.5950

Full-matrix least-squares on $\mathrm{F}^{2}$ $5218 / 9 / 247$

$\mathrm{R} 1=0.0455, \mathrm{wR} 2=0.0920$

$\mathrm{R} 1=0.0600, \mathrm{wR} 2=0.0967$

$\mathrm{n} / \mathrm{a}$

1.041 and $-1.091 \mathrm{e}^{-3}$ 


\subsection{2. $\left[\operatorname{Ir}\left\{\mathrm{N}\left(\mathrm{CHCHP}^{t} \mathrm{Bu}_{2}\right)_{2}\right\}\left(\mathrm{NH}_{3}\right)\right]\left[\mathrm{BF}_{4}\right](20)$}

Empirical formula

Formula weight

Temperature

Wavelength

Crystal system, space group

Unit cell dimensions

Volume

Z, Calculated density

Absorption coefficient

$\mathrm{F}(000)$

Crystal size

$\theta$ range for data collection

Index ranges

Reflections collected

Independent reflections

Completeness to $\theta$

Refinement method

Data / restraints / parameters

Goodness-of-fit on $\mathrm{F}^{2}$

Final $\mathrm{R}$ indices $[\mathrm{I}>2 \sigma(\mathrm{I})]$

$\mathrm{R}$ indices (all data)

Largest diff. peak and hole
C24 H51 B F4 Ir N2 O P2

724.62

100(2) K

$0.56086 \AA$

Monoclinic, $\mathrm{P} 2{ }_{1} / \mathrm{n}$

$\mathrm{a}=10.9990(13) \AA \alpha=90^{\circ}$

$\mathrm{b}=19.523(2) \AA \beta=105.086(4)^{\circ}$

$\mathrm{c}=15.0830(19) \AA \gamma=90^{\circ}$

$3127.2(7) \AA^{3}$

$4,1.539 \mathrm{Mg} / \mathrm{m}^{3}$

$2.402 \mathrm{~mm}^{-1}$

1460

? $\mathrm{x} ? \mathrm{x} ? \mathrm{~mm}^{3}$

1.65 to $26.61^{\circ}$.

$-13 \leq \mathrm{h} \leq 17,-31 \leq \mathrm{k} \leq 29,-24 \leq \mathrm{l} \leq 16$

32439

$13276[\mathrm{R}(\mathrm{int})=0.0487]$

$=26.6199 .4 \%$

Full-matrix least-squares on $\mathrm{F}^{2}$

13276 / $95 / 330$

1.029

$\mathrm{R} 1=0.0472, \mathrm{wR} 2=0.1076$

$\mathrm{R} 1=0.0775, \mathrm{wR} 2=0.1170$

3.165 and $-1.394 \mathrm{e}^{-3}$ 


\subsection{3. $\left[\operatorname{Ir}\left\{\mathrm{N}\left(\mathrm{CHCHP}^{t} \mathrm{Bu}_{2}\right)_{2}\right\}\left(\mathrm{NH}_{2}\right)\right] \mathrm{SbF}_{6}(21)$}

Empirical formula

Formula weight

Temperature

Wavelength

Crystal system, space group

Unit cell dimensions

Volume

Z, Calculated density

Absorption coefficient

$\mathrm{F}(000)$

Crystal size

$\theta$ range for data collection

Limiting indices

Reflections collected / unique

Completeness to $\Theta=19.665$

Absorption correction

Max. and min. transmission

Refinement method

Data / restraints / parameters

Goodness-of-fit on $\mathrm{F}^{2}$

Final R indices $[\mathrm{I}>2 \sigma(\mathrm{I})]$

$\mathrm{R}$ indices (all data)

Extinction coefficient

Largest diff. peak and hole
C20 H42 F6 Ir N2 P2 Sb

800.44

100(2) K

$0.56086 \AA$

Monoclinic, P 21/c

$\mathrm{a}=7.7625(19) \AA \alpha=90^{\circ}$

$\mathrm{b}=28.319(7) \AA \beta=103.127(3)^{\circ}$

$\mathrm{c}=12.879(3) \AA \gamma=90^{\circ}$

$2757.0(12) \mathrm{A}^{3}$

$4,1.928 \mathrm{Mg} / \mathrm{m}^{3}$

$3.232 \mathrm{~mm}^{-1}$

1552

$0.14 \times 0.13 \times 0.09 \mathrm{~mm}^{3}$

1.135 to $20.952^{\circ}$

$-9 \leq \mathrm{h} \leq 9,-36 \leq \mathrm{k} \leq 36,-16 \leq \mathrm{l} \leq 16$

$80791 / 5961$ [R(int $)=0.0408]$

$100.0 \%$

Semi-empirical from equivalents

0.7445 and 0.6256

Full-matrix least-squares on $\mathrm{F}^{2}$

$5961 / 98 / 309$

1.106

$\mathrm{R} 1=0.0185, \mathrm{wR} 2=0.0350$

$\mathrm{R} 1=0.0205, \mathrm{wR} 2=0.0358$

$\mathrm{n} / \mathrm{a}$

1.144 and $-1.001 \mathrm{eA}^{-3}$ 


\subsection{4. $\left[\operatorname{Ir}\left\{\mathrm{N}\left(\mathrm{CHCHP}^{t} \mathrm{Bu}_{2}\right)_{2}\right\}\left(\mathrm{NH}_{2}\right)\right](24)$}

Empirical formula

Formula weight

Temperature

Wavelength

Crystal system, space group

Unit cell dimensions

Volume

Z, Calculated density

Absorption coefficient

$\mathrm{F}(000)$

Crystal size

$\theta$ range for data collection

Limiting indices

Reflections collected / unique

Completeness to $\theta=29.62$

Absorption correction

Max. and min. transmission

Refinement method

Data / restraints / parameters

Goodness-of-fit on $\mathrm{F}^{2}$

Final R indices $[\mathrm{I}>2 \sigma(\mathrm{I})]$

$\mathrm{R}$ indices (all data)

Largest diff. peak and hole
C20 H42 Ir N2 P2

564.70

100(2) K

$0.71073 \AA$

Triclinic, $\mathrm{P} \overline{1}$

$\mathrm{a}=8.4131(5) \AA \quad \alpha=79.3270(10)^{\circ}$

$\mathrm{b}=11.6567(7) \AA \beta=89.5590(10)^{\circ}$

$\mathrm{c}=13.0591(8) \AA \gamma=69.0310(10)^{\circ}$

$1172.79(12) \mathrm{A}^{3}$

2, $1.599 \mathrm{Mg} / \mathrm{m}^{3}$

$5.835 \mathrm{~mm}^{-1}$

566

$0.11 \times 0.08 \times 0.07 \mathrm{~mm}^{3}$

1.91 to $29.62^{\circ}$

$-8 \leq \mathrm{h} \leq 11,-15 \leq \mathrm{k} \leq 16,-18 \leq \mathrm{l} \leq 18$

$24697 / 6590[\mathrm{R}(\mathrm{int})=0.0273]$

$99.6 \%$

Semi-empirical from equivalents

0.746 and 0.671

Full-matrix least-squares on $\mathrm{F}^{2}$

$6590 / 0 / 244$

1.039

$\mathrm{R} 1=0.0163, \mathrm{wR} 2=0.0361$

$\mathrm{R} 1=0.0183, \mathrm{wR} 2=0.0369$

1.196 and $-0.615 \mathrm{e}^{-3}$ 


\subsection{5. $\left[\operatorname{Ir}\left\{\mathrm{N}\left(\mathrm{CHCHP}^{t} \mathrm{Bu}_{2}\right)_{2}\right\}(\mathrm{NO})\right] \mathrm{PF}_{6}(25)$}

Empirical formula

Formula weight

Temperature

Wavelength

Crystal system, Space group

Unit cell dimensions

Volume

Z, Calculated Density

Absorption coefficient

$\mathrm{F}(000)$

Crystal size

$\theta$ range for data collection

Index ranges

Reflections collected

Independent reflections

Completeness to $\theta=19.665^{\circ}$

Refinement method

Data / restraints / parameters

Goodness-of-fit on $\mathrm{F}^{2}$

Final R indices $[\mathrm{I}>2 \sigma(\mathrm{I})]$

$\mathrm{R}$ indices (all data)

Extinction coefficient

Largest diff. peak and hole
C10 H20 F3 Ir0.50 N O0.50 P1.50

361.82

100(2) K

$0.56086 \AA$

Monoclinic, P 21/m

$\mathrm{a}=9.948(5) \AA \alpha=90^{\circ}$

$\mathrm{b}=11.929(6) \AA \quad \beta=101.00(2)^{\circ}$ $\mathrm{c}=11.396(5) \mathrm{A} \AA \quad \gamma=90^{\circ}$

$1327.5(11) \AA^{3}$

4, $1.810 \mathrm{Mg} / \mathrm{m}^{3}$

$2.862 \mathrm{~mm}^{-1}$

716

$0.15 \times 0.10 \times 0.05 \mathrm{~mm}^{3}$

1.436 to $21.996^{\circ}$

$-13 \leq \mathrm{h} \leq 13,-15 \leq \mathrm{k} \leq 15,-15 \leq \mathrm{l} \leq 15$

40707

$3458[\mathrm{R}(\mathrm{int})=0.0473]$

$$
100.0 \%
$$

Full-matrix least-squares on F2

$3458 / 13 / 175$

1.076

$\mathrm{R} 1=0.0162, \mathrm{wR} 2=0.0348$

$\mathrm{R} 1=0.0195, \mathrm{wR} 2=0.0356$

$\mathrm{n} / \mathrm{a}$

0.611 and $-0.880 \mathrm{e}^{-3}$ 


\subsection{6. $\left[\operatorname{Ir}\left\{\mathrm{N}\left(\mathrm{CHCHP}^{t} \mathrm{Bu}_{2}\right)_{2}\right\}(\mathrm{NS})\right] \mathrm{PF}_{6}(26)$}

Empirical formula

Formula weight

Temperature

Wavelength

Crystal system, Space group

Unit cell dimensions

Volume

Z, Calculated Density

Absorption coefficient

$\mathrm{F}(000)$

Crystal size

$\theta$ range for data collection

Index ranges

Reflections collected

Independent reflections

Completeness to theta $=25.242^{\circ}$

Refinement method

Data / restraints / parameters

Goodness-of-fit on $\mathrm{F}^{2}$

Final R indices $[\mathrm{I}>2 \sigma(\mathrm{I})]$

$\mathrm{R}$ indices (all data)

Extinction coefficient

Largest diff. peak and hole
C20 H40 F6 Ir N2 P3 S

739.71

100(2) K

$0.71073 \AA$

Monoclinic, P 21/m

$\mathrm{a}=10.1144(5) \AA \alpha=90^{\circ}$

$\mathrm{b}=12.0938(6) \AA \beta=100.3720(10)^{\circ}$

$\mathrm{c}=11.3497(5) \AA \gamma=90^{\circ}$

$1365.63(11) \AA^{3}$

2, $1.799 \mathrm{Mg} / \mathrm{m}^{3}$

$5.194 \mathrm{~mm}^{-1}$

732

$0.099 \times 0.079 \times 0.054 \mathrm{~mm}^{3}$

1.824 to $28.300^{\circ}$

$-13 \leq \mathrm{h} \leq 13,-16 \leq \mathrm{k} \leq 16,-15 \leq \mathrm{l} \leq 15$

19965

$3551[\mathrm{R}($ int $)=0.0250]$

$100.0 \%$

Full-matrix least-squares on F2

$3551 / 13 / 176$

1.048

$\mathrm{R} 1=0.0148, \mathrm{wR} 2=0.0360$

$\mathrm{R} 1=0.0154, \mathrm{wR} 2=0.0362$

$\mathrm{n} / \mathrm{a}$

1.205 and -0.628 e. $\AA^{-3}$ 


\subsection{7. $\left[\operatorname{Ir}\left\{\mathrm{N}\left(\mathrm{CHCHP}{ }^{t} \mathrm{Bu}_{2}\right)_{2}\right\}(\mathrm{NSe})\right] \mathrm{PF}_{6}$ (27)}

Empirical formula

Formula weight

Temperature

Wavelength

Crystal system, space group

Unit cell dimensions

Volume

Z, Calculated density

Absorption coefficient

$\mathrm{F}(000)$

Crystal size

$\theta$ range for data collection

Limiting indices

Reflections collected / unique

Completeness to $\Theta=19.665$

Absorption correction

Max. and min. transmission

Refinement method

Data / restraints / parameters

Goodness-of-fit on $\mathrm{F}^{2}$

Final $\mathrm{R}$ indices $[\mathrm{I}>2 \sigma(\mathrm{I})]$

$\mathrm{R}$ indices (all data)

Extinction coefficient

Largest diff. peak and hole
C20 H40 F6 Ir N2 P3 Se

786.61

100(2) K

$0.56086 \AA$

Monoclinic, C 2/c

$$
\mathrm{a}=21.602(2) \AA \alpha=90^{\circ}
$$

$\mathrm{b}=13.3790(10) \AA \beta=98.01(4)^{\circ}$

$\mathrm{c}=19.8950(10) \AA \gamma=90^{\circ}$

5693.8(9) $\AA^{3}$

$8,1.835 \mathrm{Mg} / \mathrm{m}^{3}$

$3.344 \mathrm{~mm}^{-1}$

3072

$0.250 \times 0.200 \times 0.090 \mathrm{~mm}^{3}$

1.417 to $23.754^{\circ}$

$-30 \leq \mathrm{h} \leq 30,-19 \leq \mathrm{k} \leq 19,-28 \leq \mathrm{l} \leq 27$

$62762 / 8770[\mathrm{R}($ int $)=0.0744]$

$100.0 \%$

Multiscan

0.753 and 0.489

Full-matrix least-squares on $\mathrm{F}^{2}$

$8770 / 2374 / 554$

1.064

$\mathrm{R} 1=0.0352, \mathrm{wR} 2=0.0799$

$\mathrm{R} 1=0.0498, \mathrm{wR} 2=0.0846$

$\mathrm{n} / \mathrm{a}$

2.771 and -2.158 e $\AA^{-3}$ 


\section{List of scientific contributions}

\section{Publications in scientific journals}

1. J. Meiners, M. G. Scheibel, M.-H. Lemée-Cailleau, S. A. Mason, M. B. Boeddinghaus, T. F. Fässler, E. Herdtweck, M. M. Khusniyarov, S. Schneider, Angew. Chem. Int. Ed. 2011, 50, 8184-8187.

2. M. G. Scheibel, S. Schneider, Angew. Chem. Int. Ed. 2012, 51, 2-5.

3. M. G. Scheibel, B. Askevold, F. W. Heinemann, E. J. Reijerse, B. de Bruin, S. Schneider, Nature Chem. 2012, 4, 552-558.

4. M. G. Scheibel, I. Klopsch, H. Wolf, P. Stollberg, D. Stalke, S. Schneider, Eur. J. Inorg. Chem. 2013, 3453-3457.

5. M. G. Scheibel, Y. Wu, A. C. Stückl, L. Krause, E. Carl, D. Stalke, B. de Bruin, S. Schneider, J. Am. Chem. Soc. 2013, 135, 17719-17722.

6. M. Kinauer, M. G. Scheibel, J. Abbenseth, F. W. Heinemann, P. Stollberg, C. Würtele, S. Schneider, Dalton Trans. 2014, 43, 4506-4513.

\section{Oral contributions to conferences}

1. M. G. Scheibel, M. M. Khusniyarov, B. de Bruin, S. Schneider, "Square-planar iridium(I-V) PNP pincer complexes: Closed and open shell iridium nitrides", 243rd ACS National Meeting, San Diego/USA, 2012.

2. M. G. Scheibel, B. Askevold, M. M. Khusniyarov, F. W. Heinemann, E. J. Reijerse, B. de Bruin, S. Schneider, "Closed-Shell and Open-Shell Square-Planar Iridium Nitrido Complexes", 15. Northern German PhD Colloquium, Krelingen/Germany, 2012. 
3. M. G. Scheibel, E. J. Reijerse, B. de Bruin, S. Schneider, "Closed-Shell and OpenShell Square-Planar Iridium Nitrido Complexes", 9th Coordination Chemistry Meeting, Bayreuth/Germany, 2013, awarded.

4. M. G. Scheibel, M. Kinauer, B. de Bruin, S. Schneider, "Parent iridium amido complexes", Catalytic Routines for Small Molecule Activation (CARISMA), Venice/Italy, 2014 .

\section{Poster presentations on conferences}

1. M. G.Scheibel, J. Meiners, S. Schneider, "Square-plana iridium $d^{8}, d^{7}, d^{6}$ and $d^{4}$ complexes stabilized by a PNP pincer ligand, 3rd Erlangen Symposium on Redox Active Metal Complexes, Erlangen/Germany, 2011.

2. M. G. Scheibel, F. W. Heinemann, E. J. Reijerse, B. de Bruin, S. Schneider, "Closed-Shell and Open-Shell Square-Planar Iridium Nitrido Complexes", 16. Wöhler Tagung, Göttingen/Germany, 2012.

3. M. G. Scheibel, F. W. Heinemann, E. J. Reijerse, B. de Bruin, S. Schneider, "Closed-Shell and Open-Shell Square-Planar Iridium Nitrido Complexes", Lower Saxony Catalysis Symposium, Göttingen/Germany 2012.

4. M. G. Scheibel, E. J. Reijerse, B. de Bruin, S. Schneider, "Closed-shell and openshell group 9 transition metal nitrido complexes", 9th International School of Organometallic Chemistry, Camerino/Italy, 2013. 


\section{Curriculum vitae}

\section{Personal}

$\begin{array}{ll}\text { Name } & \text { Markus Scheibel } \\ \text { Date of birth } & 28.05 .1985 \\ \text { Place of birth } & \text { Munich } \\ \text { Citizenship } & \text { German } \\ \text { Parents } & \text { Wolfgang Scheibel } \\ & \text { Agnes Scheibel }\end{array}$

\section{Education}

$1996-2005$

Gymnasium Kirchheim

$2005-2008$

Studies Bachelor of Science (Chemistry)

Technical University Munich

Bachelor Thesis with Prof. Dr. Johann Planck

$2008-2010$

Studies Master of Science (Chemistry)

Technical University Munich

Master Thesis with Prof. Dr. Sven Schneider

2010 - $2011 \quad$ Dissertation at Technical University of Munich

Supervisor: Prof. Dr. Sven Schneider

2011 - $2012 \quad$ Dissertation at Friedrich Alexander University Erlangen-Nuremberg Supervisor: Prof. Dr. Sven Schneider

since May 2012 Dissertation at Georg-August University Göttingen

Supervisor: Prof. Dr. Sven Schneider 


\section{Internships}

July 2010

External practical course at King Abdullah University of Science and Technology

Supervisor: Prof. Dr. Jörg Eppinger

\section{Languages}

German, English, French 